\title{
Remedial Investigation Plan for Waste Area Grouping 1 at Oak Ridge National Laboratory, Oak Ridge, Tennessee: Responses to Regulator Comments
}




\title{
Remedial Investigation Plan for Waste Area Grouping 1 at Oak Ridge National Laboratory, Oak Ridge, Tennessee: Responses to Regulator Comments
}

\author{
Environmental Restoration Division \\ P.O. Box 2003 \\ Oak Ridge, Tennessee 37831-7256
}

Date Issued-May 1991

\author{
Prepared by \\ Bechtel National, Inc./CH2M HILL/ERCE/PEER \\ Oak Ridge, Tennessee \\ under subcontract 30B-99053C
}

Prepared for

U.S. Department of Energy

Office of Environmental Restoration and Waste Manafsement

under budget and reporting code EW 20

MARTIN MARIETTA ENERGY SYSTEMS, INC.

monaging the

Oak Ridge National Laboratory

Oak Ridge K-25 Site
Oak Ridge Y-12 Plant

Paducah Gaseous Diffusion Plant

under contract DE-AC05-84OR21400

and the

Portsmouth Gaseous Diffusion Plant

under contract DE-AC05-76OR00001

for the

U.S. DEPARTMENT OF ENERGY 


\section{Bechtel National, Inc.CCH2M HILL•ERCE•PEER Oak Ridge, Tennessee}

contributed to the preparation of this document and should not be considered eligible contractors for its review.

This report has been reproduced directly from the best avallable copy.

Avallable to DOE and DOE contractors from the Oilice of Scientific and Technical Information, P.O. Box 62, Oak Ridge, TN 37831; prices available from 615-576-8401, FTS 626-8401

Avallable to the public from the National Technical Information Service, U.S. Department of Commerce, 5285 Port Royal Rd., Springfield, VA 22161 and from the INFORMATION RESOURCE CENTER for the Environmental Restoration Program, 105 Broacway, Oak Ridge, TN 37830 
ES/ER-6\&D2

ORNL/ER/Sub-87/99053/4\&R2

\section{INTERNAL DISTRIBUTION}

1. T. L. Ashwood

2. L. D. Bates

3. H. M. Braunstein

4. T. W. Burwinkle

5. K. W. Cook

6. N. W. Durfee

7. S. B. Garland II

8-9. L. F. Goins

10. D. D. Huff

11. L. D. Hyde

12. L. L. Kaiser

13. A. J. Kuhaida, Jr.

14. J. R. Lawson
15. L. E. McNeese

16. C. E. Nix

17. L. B. Oakley

18-19. P. T. Owen

20. J. A. Reafsnyder

21. D. W. Swindle

22. L. D. Voorhees

23. R. K. White

24. A. S. Will III

25. Central Research Library

26-47. ER Document Management Center

48-49. Laboratory Records Department

50. Laboratory Records, ORNL-RC

\section{EXTERNAL DISTRIBUTION}

51. Office of Assistant Manager for Energy Research and Development, Department of Energy, Oak Ridge Operations, P.O. Box 2001, Oak Ridge, TN 37831-8600

52. P. H. Edmonds, Lee Wan and Associates, 105 Broadway, Oak Ridge, TN 37830

53. L. O. Pendergraft, Labat-Anderson, Inc., Information Resource Center, Administrative Record File, 105 Broadway, Oak Ridge, TN 37830

54-55. S. P. Riddle, Department of Energy, Oak Ridge Operations, P.O. Box 2001, Oak Ridge, TN 37831-8621

56-75. R. C. Sleeman, Director, Environmental Restoration Division, Department of Energy, Oak Ridge Operations, P.O. Box 2001, Oak Ridge, TN 37831-8541

76-87. Office of Scientific and Technical Information, P.O. Box 62, Oak Ridge, TN 37831 


\section{CONTENTS}

Page

ACRONYMS $\ldots \ldots \ldots \ldots \ldots \ldots \ldots \ldots \ldots \ldots \ldots \ldots \ldots \ldots \ldots$

EXECUTIVE SUMMARY $\ldots \ldots \ldots \ldots \ldots \ldots \ldots \ldots \ldots$ vi

LIST OF ATTACHMENTS $\ldots \ldots \ldots \ldots \ldots \ldots \ldots \ldots \ldots$ vii

\section{SECTION I-CERCLA COMMENTS}

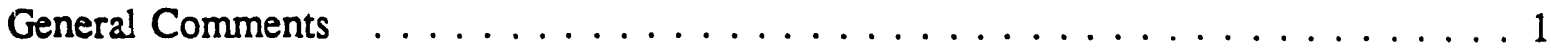

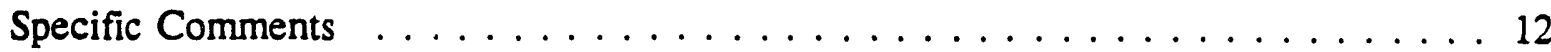

SECTION II-RCKA COMMENTS

Part A

General Comments . . . . . . . . . . . . . . . . . . . . . . . . 77

Specific Comments . . . . . . . . . . . . . . . . . . 82

$\underline{\text { Part B }}$

Specific Comments . . . . . . . . . . . . . . . . . . 106

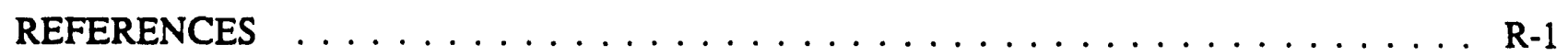

\section{APPENDIX}

Attachment A-CERCLA Supporting Documentation . . . . . . . . . A-1

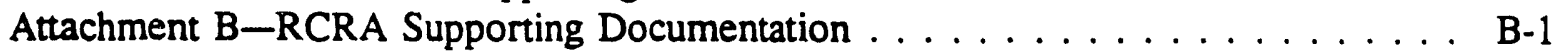




\section{ACRONYMS}

ARAR

ATSDR

BMAP

CERCLA

CLP

CSL

DOE

EM

EP

EPA

ES\&H

ESP

FFA

FHS

FSP

FS

FSS

GC

GPR

ICP

ILW

LLW

LLLW

NFA

NPL

ORNL

ORR

PCB

PVC

QA

QAA

QAPP

QC

RCRA

RFI

RI

ROD

SAS

SITE

SOP

SWMU

SWSA applicable or relevant and appropriate requirement

Agency for Toxic Substances and Disease Registry

Biological Monitoring and Abatement Program

Comprehensive Environmental Response, Compensation and Liability

Act

Contract Laboratory Program

close support laboratory

Department of Energy

electromagnetic

extraction procedure

Environmental Protection Agency

Environmental, Safety and Health

Environmental Surveillance Procedures

Federal Facility Agreement

field health and safety

field sampling plan

Feasibility Study

field services and support

gas chromatograph

ground penetrating radar

inductively coupled plasma

intermediate-level waste

low-level waste

liquid low-level waste

no further action

National Priority List

Oak Ridge National Laboratory

Oak Ridge Reservation

polychlorinated biphenyl

polyvinylchloride

quality assurance

quality assurance assessment

Quality Assurance Project Plan

quality control

Resource Conservation and Recovery Act

RCRA Facility Investigation

Remedial Investigation

record of decision

statistical analysis system

Superfund Innovative Technology Evaluation

standard operating procedure

solid waste management unit

solid waste storage area 
TAL

TBC

TCL

TCLP

TDC

TDHE

TDS

TIM

TRU

USRADS

VOC

WAG

WOC

$\mathrm{XRF}$ target analyte list

to be considered

target compound list

toxicity characteristics leaching procedure

Tennessee Department of Conservation

Tennessee Department of Health and Environment total dissolved solids

Technical Integration Manager

transuranic

Ultrasonic Ranging and Data Systems

volatile organic compound

waste area grouping

White Oak Creek

$\mathrm{X}$-ray fluorescence 


\section{EXECUTIVE SUMMARY}

This document, ES/ER-6\&D2, is a companion document to ORNL/RAP/Sub87/99053/4\&R1, Remedial Investigation Plan for ORNL Waste Area Grouping 1, dated August 1989. This document lists comments received from the Environmental Protection Agency, Region IV (EPA) and the Tennessee Department of Health and Environment (TDHE) ${ }^{1}$ and responses to each of these comments. As requested by EPA, a revised Remedial Investigation (RI) Plan for Waste Area Grouping (WAG) 1 will not be submitted.

The document is divided into two Sections and an Appendix. Section I contains responses to comments issued on May 22, 1990, by EPA's Region IV program office responsible for implementing the Comprehensive Environmental Response, Compensation, and Liability Act (CERCLA). Section II contains responses to comments issued on April 7, 1989, by EPA's program office responsible for implementing the Resource Conservation and Recovery Act (RCRA); these comments include issues raised by the TDHE. The Appendix contains the attachments referenced in a number of the responses.

${ }^{1}$ Since reorganized as the Tennessee Department of Conservation (TDC). 


\section{LIST OF ATTACHMENTS}

Attachment A - CERCLA Comments

Specific

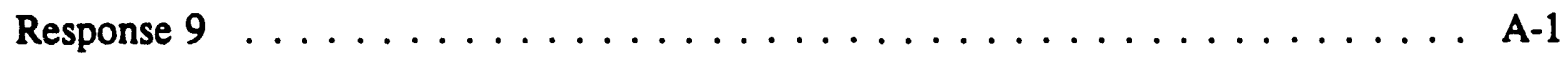

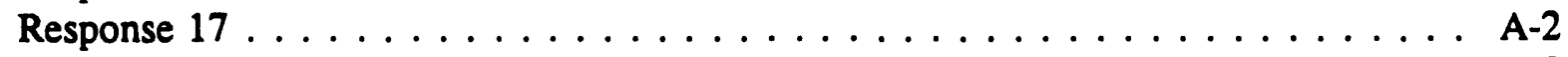

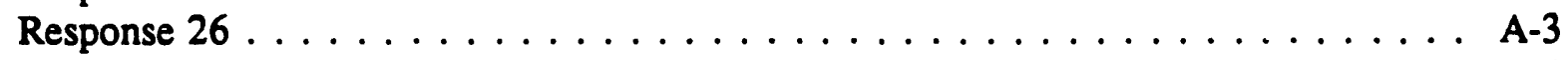

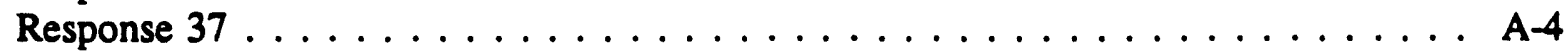

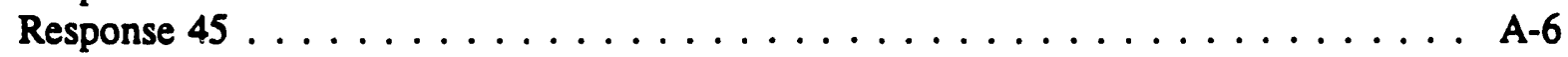

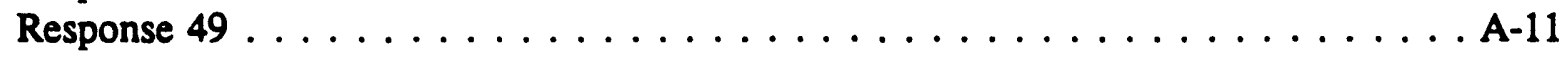

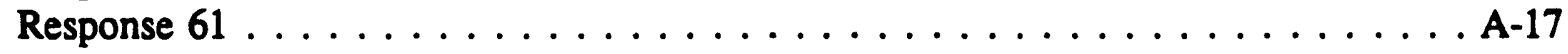

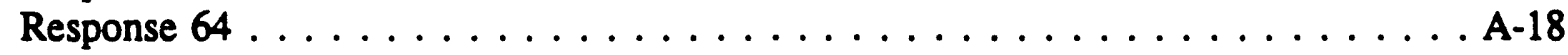

Response $66 \ldots \ldots \ldots \ldots \ldots \ldots \ldots \ldots \ldots \ldots$. . . . . . . . . . . . . . . . . . . . . . . . . . .

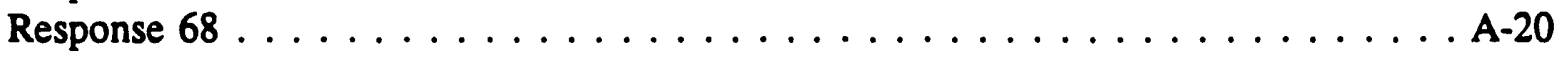

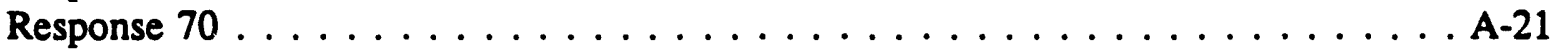

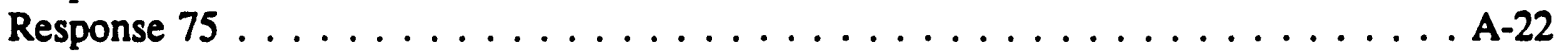

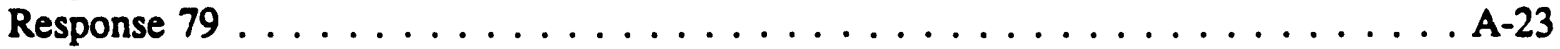

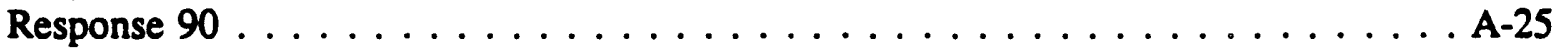

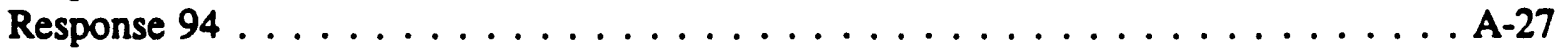

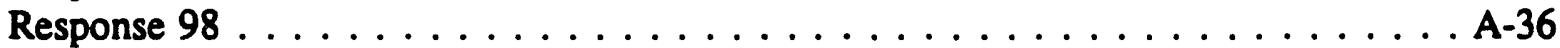

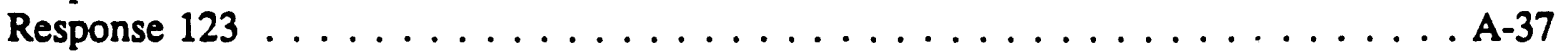

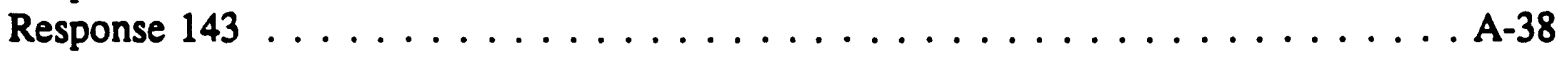

Attachment B - RCRA Comments

Part A: General

Response $6 \ldots \ldots \ldots \ldots \ldots \ldots \ldots \ldots \ldots \ldots \ldots \ldots \ldots \ldots \ldots$

Part A: Specific

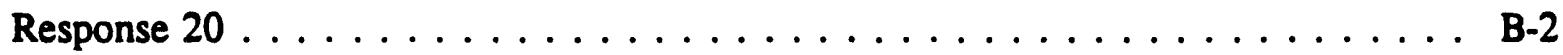

Response $58 \ldots \ldots \ldots \ldots \ldots \ldots \ldots \ldots \ldots \ldots \ldots \ldots \ldots$

Part B: Specific

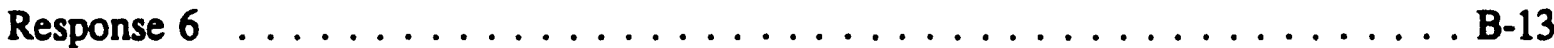

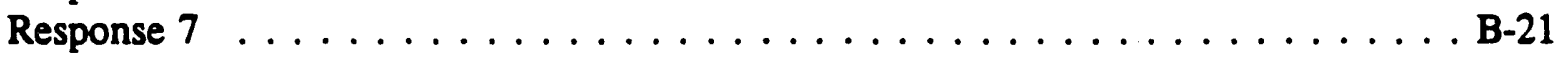

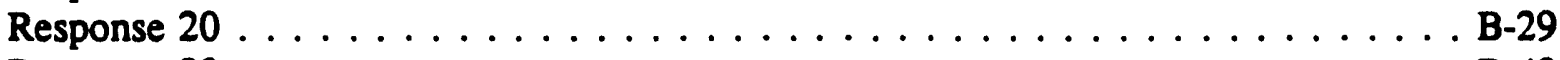

Response $23 \ldots \ldots \ldots \ldots \ldots \ldots \ldots \ldots \ldots \ldots \ldots \ldots \ldots$. . . . . . . . . . . . . . . . . . . . . . . . . . . . . .

Response $33 \ldots \ldots \ldots \ldots \ldots \ldots \ldots \ldots \ldots \ldots \ldots \ldots$. . . . . . . . . . . . . . . . . . . . .

Response $34 \ldots \ldots \ldots \ldots \ldots \ldots \ldots \ldots \ldots \ldots \ldots \ldots \ldots$. . . . . . . . . . . . . . . . . . . . . . . . . .

Response $36 \ldots \ldots \ldots \ldots \ldots \ldots \ldots \ldots \ldots \ldots \ldots \ldots \ldots$. . . . . . . . . . . . . . . . . .

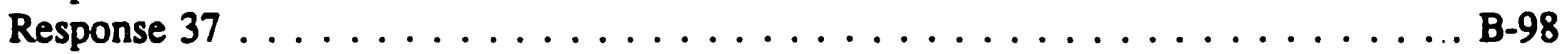

Response $38 \ldots \ldots \ldots \ldots \ldots \ldots \ldots \ldots \ldots \ldots \ldots$. . . . . . . . . . . . . . . . 


\title{
RESPONSE TO CERCLA COMMENTS ON WAG 1 RI PLAN
}

\author{
GENERAL COMMENTS
}

\section{INTRODUCTION}

The following section contains responses to CERCLA comments on the ORNL WAG 1 RI Plan issued by EPA's Region IV (Atlanta, Georgia) office on May 22, 1990. Supporting documentation (graphics, tables, procedures, etc.) is contained in Attachment A. This documentation is presented in the same order as the comment.

\section{COMMENT 1:}

While the RI Phase I Work Plan proposed by the RI Plan is in general adequate for scoping purposes, it will not meet the CERCLA requirements for Operable Unit-based Feasibility Studies. According to CERCLA, the RI and FS are integrated, with the FS data needs influencing RI scoping requirements. Therefore, it is not appropriate to delay the start of the FS until after the RI has been completed as proposed by the Plan. Based on RI Phase I conclusions, the WAG-1 RI Plan should provide for Feasibility Studies to be conducted concurrently, to the degree possible, with appropriate RI Phase II work.

\section{RESPONSE}

Site characterization data will be evaluated during Phase I, using the "Observational Approach" to identify discrete operable units that can contribute to the overall remedial action goals. During Phase II, specific operable unit feasibility studies will be conducted when the data collected during Phase I provide adequate support. Otherwise, additional data will be collected through operable unit-specific RIs. Opportunities for removal actions will be identified during both Phase I and II.

\section{COMMENT 2:}

It is difficult to get a complete picture of the specifics of the RI tasks because of the generalized structure and broad scope of the Plan. The WAG-1 task descriptions in Section 2.4 are very generic in nature and can just as well be for any other WAG. The reader must get to Section 5, "Technical Approach ...", and Appendix A, "Field Sampling Plan" to obtain specifics of the tasks to be carried out at WAG-1. The RI Plan should therefore be more focused on WAG-1 specifics throughout. 


\section{RESPONSE}

The intent of Section 2.4 , as stated in the introductory paragraph, is to summarize the administrative and technical tasks conducted as part of the remedial investigation, and to show how these tasks are related to a work breakdown structure. This information was provided in this way to serve the more generic needs of project management. DOE agrees that this section does not focus on the specifics of the field investigation, and that the reader must refer to later sections of the plan for this information.

The specific tasks identified in the RI Plan, when implemented in the phased approach, will provide a thorourh characterization of the site to support remedial action decision making.

\section{COMMENT 3:}

To satisfy CERCLA requirements, Operable Unit-based RI Phase II/FS Work Plans should be developed based on the findings of the proposed RI Phase I work and submitted to EPA and TDHE for review and comment.

\section{RESPONSE}

Operable unit-based RI Phase II/FS Work Plans will be submitted for review and comment.

\section{COMMENT 4:}

Existing information combined with the proposed RI Phase I work should provide the basis for the development of a Preliminary Characterization Summary Report and a Preliminary Risk Assessment Report.

The above reports will be used by EPA and TDHE to determine the need for, and the scope of RI Phase II work, including any necessary Operable Unit-specific Feasibility Studies and the initial identification of Operable Unit-specific ARARs.

\section{RESPONSE}

DOE will submit a Preliminary Charactorization Summary Report and a Preliminary Risk Assessment Report at the conclusion of Phase I. DOE will also identify preliminary operable units and make recommendations regarding their disposition, i.e., NFA, FS/ROD, or Phase II RI. 


\section{COMMENT 5:}

Based on the review of the Preliminary Characterization Summary Report and Preliminary Risk Assessment Report, if the potential risk is high for the study area or portion thereof, and the No Further Action alternative is not supported, as part of RI Phase II activities, Operable Unit-specific risk characterization should be developed as outlined in the "Risk Assessment Guidance for Superfund, Volume 1: Human Health Evaluation Manual", September 1989, OSWER Directive 9285.7-01a, the "Risk Assessment Guidance for Superfund, Volume II: Environmental Evaluation Manual", March 1989, OSWER Directive 9285.7-02 and the "Superfund Exposure Assessment Manual", April 1988, OSWER Directive 9285.5-1.

In particular, research information concerning hazard identification and dose-response studies must be obtained to ascertain the actual risk posed by a specific Operable Unit.

\section{RESPONSE}

DOE will supplement published data with the results of ongoing research studies. Existing studies and programs [(i.e., from the Biological Monitoring and Abatement Program (BMAP)] will be used to aid in quantifying actual risk. Site information on hazards, risk, and dose will also be used to assess actual risk posed by operable units.

\section{COMMENT 6:}

Hazard identification may include epidemiological and animal bioassay data. This additional information combined with the Preliminary Risk Assessment Report could satisfy CERCLA Risk Assessment requirements.

\section{RESPONSE}

Agree.

\section{COMMENT 7:}

As required by CERCLA Section 104, the RI Plan should provide for support from the Agency for Toxic Substances and Disease Registry (ATSDR) as discussed in the "Guidance for Coordinating ATSDR Health Assessment Activities with the Superfund Remedial Process", May 1987, OSWER Directive 9285.4-02. ATSDR is required to conduct a Health Assessment (HA) for every Site proposed for inclusion on the NPL.

Coordination between ATSDR and DOE is necessary considering that DOE must provide the necessary $\mathrm{HA}$ data and information. The purpose of the $\mathrm{HA}$ is to assist in determining whether current or potential immediate or long term risk to human health exists at a Site 
and whether additional information on human exposure and associated health risks is needed. The HA is required to be completed to the maximum extent practicable, before completion of the ORR RIs.

\section{RESPONSE}

The entire Oak Ridge Reservation is listed on the NPL, not just WAG 1. Information will be provided pursuant to OSWER Directive 9285.4-02.

\section{COMMENT 8:}

The Plan has been based on a partial evaluation of available existing data and information, leaving the remainder to be done at some unspecified time during the $R I$. In addition, referenced data is not sufficiently documented by the plan, or the discussion is not specific enough. Documented contamination should generally be quantified rather than qualified.

\section{RESPONSE}

Substantial evaluation of existing data and reports has occurred since this comment was written. These evaluations form the basis of the current site conceptual model, and are being used to redesign sampling campaigns, where appropriate. All existing information will be used in addressing the objectives of the RI process, including the nature and extent of contamination, risk assessment, site characterization, waste and release characterization, and remedial action opportunities.

There are a number of Responses to CERCLA Specific Comments $(37,45,49,70,74,75$, 94,96 , and 175) that address the documentation, use, and interpretation of existing data. Please refer to the responses to those comments for further information.

\section{COMMENT 9:}

Section 3 needs to reference appropriate illustrative maps (topographic, surface waters, etc.) in the appendices to support the text.

\section{RESPONSE}

Additional maps on site topography, drainage patterns, geology, and site conditions are presented in Responses to CERCLA Specific Comments 64, 66, 68, and 98. 


\section{COMMENT 10:}

The proposed ORNL generic risk assessment study at the completion of RIs for all WAGs is inadequate. The RI Plan should provide for a Preliminary Risk Assessment Report at the completion of Phase I work, and a Baseline Risk Assessment report at the completion of the RI. Also, sampling and analysis of biota should be part of the RI activities to support risk assessment decisions. As part of the RI Phase I activities, the RI Plan should evaluate the ORNL monitoring (Biota) program to ensure its usefulness as an input to the RI in terms of types, numbers, location of sampling points, parameters, analytical procedures, and QAVCC requirements.

\section{RESPONSE}

A Preliminary Risk Assessment Report and a Baseline Risk Assessment Report will be propared at the conclusions of Phases I and II, respectively of the RI. The current biota monitoring program will be evaluated, as outlined in the comment, during Phase I of the RI. Results from biota studies will be included in the Preliminary Risk Assessment and Baseline Risk Assessment Reports.

\section{COMMENT 11:}

Data needs have been presented in generic fashion without a comprehensive comparison with available data to establish data gaps.

\section{RESPONSE}

As outlined in the Response to CERCLA General Comment 8, existing data have been evaluated since this comment was prepared. This evaluation serves as the basis for developing SWMU-specific sampling campaigns. Data gaps remaining after Phase I will be addressed in the Phase II Work Plan. Responses to CERCLA Specific Comments provide further information, as described in the Response to CERCLA General Comment 8.

\section{COMMENT 12:}

The Quality Assurance Project Plan (QAPP) does not include the EPA document control format required by $Q A M S-005 / 80$ in the top right hand corner of every page (section number, revision number, etc.). This could be a significant emission, since the QAPP is could be subject to many changes.[sic]

\section{RESPONSE}

The QAPP pages now follow the EPA document control format. 


\section{COMMENT 13:}

Information on reported spills or other contaminated areas undocumented by the RI Plan should be reported by the RI Plan. If quantitative information is not available, qualitative information identifying radionuclides and chemicals in the spills and contaminated areas should be described and tabulated. Where information on spills and contaminated areas is available, and corrective action was taken to minimize exposure, not only of cleanup personnel, but operations personnel as well, the nature and efficiency of such action should be documented.

\section{RESPONSE}

Section 3.2, Nature and Extent of Contamination, addresses those areas recognized as contaminated or having possible contamination (SWMUs). Pages 3-28 through 3-63 discuss the WAG 1 documented tank leaks, line leaks, spills, mercury-contaminated areas, ponds and impoundments, SWSAs, and other areas known or believed to be contaminated. In general, the amount of contaminant release is unknown, the areal extent of release is uncertain, and the depth to which migration has occurred has not been determined. An outline of the types of contaminants can be found in Table 3-10 of the WAG 1 RI Plan, Revision 1.

In addition to the SWMU approach to presenting contamination information, Sections 3.3.1.2 through 3.3.1.5 (pages 3-69 to 3-101) present media-specific contaminants. These sections include data compiled from localized media-based studies. Concentration levels and ranges are presented where known.

Corrective actions, especially at leak and spill sites, are not well documented. In a few cases (see LLW Leak at Building 3019-SWMU 1.5d), contaminated soil is known to have been removed. The excavation procedures and manner of disposal, however, are undocumented. Please refer to Attachment A, Response to CERCLA Specific Comment 49 for a table listing SWMUs and suspected contaminants.

The list of potential SWMUs is updated yearly based on ongoing investigations. Information from elements of the investigation (for example, from the radiation walkover survey using the USRADS technology) is used to pinpoint follow-up investigation locations.

In addition, project staff collect data on past spills and releases from ORNL personnel who have a detailed knowledge of plant operations to target locations for future sampling. This information is documented in a master data file, and will be used in preparing the RI Reports. During these interviews with plant personnel, information on the location, characteristics, dates, known impacts, and corrective actions of past releases is sought. 


\section{COMMENT 14:}

Although a great deal of research is performed by both the DOE (i.e., HAZRAP) and the EPA (i.e., SITE), the innovative technologies being developed in these programs are not properly incorporated into the RI Plan. DOE should consider advanced technologies (e.g., immunoassay and fiber optic techniques, and advanced geophysical techniques) for RI characterization investigations. Also, information on treatability studies under way by ORNL personnel, as it relates to specific WAG-1 concerns, should be documented in the RI Plan. The adequacy of existing and planned treatability studies should be evaluated in relation to the existing information, the proposed RI Phase I work and anticipated Feasibility Sture s. This evaluation should be documented by the Plan.

ORNL environmental restoration personnel should become familiar with treatability studies under way or completed at other national laboratories or contract-supported operations. This kind of input may be helpful in developing remedial responses under the current $R I$. Integration of treatability studies and RI activities will insure that the necessary data will be collected during the RI in support of the FS.

\section{RESPONSE}

DOE is committed to the development and application of innovative technology to meet compliance and cleanup goals. In DOE's 5-year plan for environmental restoration and waste management, corrective actions are Priority 1. Technology development is critical to achieving environmental goals. See Response to CERCLA Specific Comment 90 for a listing of technology demonstrations.

While other innovative and developmental techniques may prove valuable in later phases of the project, their application for the start of Phase $I$ is considered to be inappropriate. However, project personnel will continually review data and assess the program to identify any useful mid-course corrections.

Treatability studies are very important to achieving the overall remedial action goals of this project. The specific characteristics of the mixed wastes and releases are, perhaps, unique to ORNL. Consistent with EPA guidance, treatability studies will be a part of the Phase II RI and early FS effort. During Phase I of the RI, information on completed and ongoing treatability studies by ORNL, EPA/SITE, and other studies that may be applicable to WAG 1 concerns will be compiled.

\section{COMMENT 15:}

The collection of composite samples as described, with admixture of the materials (e.g., soil and sediments), does not permit identification of partitioning of specific nuclides or chemicals in these media. Radionuclides and chemical compounds act in accordance with the Hofmeister lyotropic series, in which multivalent elements will displace single-valent 
elements, and that evin in the same valence group a given order can be identified. Other factors affect the movement of exchanged or adsorbed nuclides or chemical elements, such as whether the particular element of compound can exist in more than one (1) ionic state (e.g., ruthenium:, or whether the nuclide or chemical is complexed or sequestered, in other words, its chemical form.

Undisturbed core samples (soil, sediments, rock), which can be evaluated to determine variable deposition based on discharges over time, would provide valuable historic information. Such information would be lost if there is admixture of the sample contents.

\section{RESPONSE}

As described in the comment, ion exchange reactions with clays and soil organic materials can affect the migration of certain elements. However, the limited nature of soil compositing presents no limitation to the detection and characterization of releases. Soil core samples will be composited over $0.6-\mathrm{m}(2-\mathrm{ft})$ intervals, not $1.8-\mathrm{m}$ intervals $(6-\mathrm{ft})$ as proposed earlier. This degree of compositing is necessary to obtain adequate sample volume for analysis. Soil or sediment samples from different iorings or collection locations will not be composited.

\section{COMMENT 16:}

Hot spots would be missed if split spoon soil and borehole samples are composited in $1.52 \mathrm{~m}$ (5-foot) intervals as proposed. It would be better to use the results of screening measurements to determine the intervals for compositing such samples rather than a fixed $1.52 m(5-f t)$ interval.

At all known areas of surface contamination, composite samples of the first $0.6 \mathrm{~m}$ (2-ft) should be analyzed as appropriate. However, in some cases it may be necessary to determine contaminant concentrations within the upper six inches.

\section{RESPONSE}

As stated in the Response to CERCLA General Comment 15, soil core compositing will be limited to $0.6-\mathrm{m}(2-\mathrm{ft})$ intervals. Field screening measurements for radionuclides and VOCs are also performed over the entire length of each soil core as it is collected; these measurements are used to identify additional, discrete sampling intervals.

\section{COMMENT 17:}

The chemical composition of the waste (e.g., pH) or any changes in its composition will affect movement of past and/or present exchanged or adsorbed radionuclides and chemical compounds. Any changes in waste composition should be duly noted in terms of their possible effects on previously deposited materials. 


\section{RESPONSE}

Available data on variations in waste composition, such as $\mathrm{pH}$, will be used in this characterization.

\section{COMMENT 18:}

The half-lives of the radionuclides will determine the radiological significance of past spills and contaminated areas. Many of the identified radionuclides, unless currently discharged, will have decayed to insignificant levels. The radionuclides of major interest are Co-60, the transuranics, uranium, radium, and plutonium. These radionuclides are of some concern with reference to the future life of the Laboratory. Dates identifying leaks and their repair should be noted to assess potential radionuclides which may still pose a hazard.

\section{RESPONSE}

While the comment is true, cesium, strontium, thorium, and tritium are also of concern and they are routinely incorporated into the sampling plans along with the non-isotope-specific parameters gross alpha and gross beta/gamma.

Dates of leaks, to the extent known, are included in the RI Plan and in ongoing data gathering efforts.

\section{COMMENT 19:}

Control radiation measurements of soils and plants should be taken in areas having similar plants and soils to those at the Laboratory site.

\section{RESPONSE}

ORNL routinely collects data on soils and flora from background areas to prepare its annual environmental surveillance report. These data are used in evaluating biota impacts at WAG 1 . Refer to Attachment A, Response to CERCLA Specific Comment 45 for analyses of grasses collected on the ORR.

\section{COMMENT 20:}

A check of the ratios of certain combinations of cations, anions, radionuclides, etc., will disclose whether the change in concentration of a given contaminant is due to dilution alone, or whether other physical, chemical, or biological factors are involved. Materials will be adsorbed or exchanged until equilibrium conditions are established. With increased concentrations, further uptake to a new equilibrium value will take place; with a decrease in 
concentration there will be a release of contaminant from the medium until a new equilibrium is established.

\section{RESPONSE}

Agree.

\section{COMMENT 21:}

In comparing past data with present data, there is a need to identify the past analytical procedures and compare these procedures with current ones with respect to accuracy, precision, minialum detection level, etc. The presence of interfering substances should be evaluated in methods used. If at all possible, chemical form should be identified, e.g., whether the substance is organic, inorganic, complexed, or in cationic, anionic, or nonionic form, etc.

\section{RESPONSE}

This process will be followed to the extent that data on previous studies are available. However, the fact that different analytical procedures may have been used in various studies, compared with current protocols, does not in any way invalidate the results. Many of the analytical results contained in existing ORNL reports on release investigations were generated by the ORNL Analytical Chemistry Division, which is a leader in radioactive and mixed waste sample analysis.

It should be possible to compare many of the previous ORNL studies with joint analyses performed on both environmental samples and matrix spike samples to develop a basis for comparison of data generated by the ORNL laboratory with analyses performed by the RI analytical subcontract laboratory. The extent to which current laboratory protocols are comparable to those used to generate the earlier data will also be assessed. This will provide a basis upon which previous ORNL reports can be used in characterizing the nature and extent of contamination and associated risks, in keeping with EPA's recent "Guidance for Data Useability in Risk Assessment", October 1990, OSWER Directive 9285.7-05.

\section{COMMENT 22:}

Exposure determination is a very complex matter, particularly in situations dealing with radionuclides and chemical compounds in a variety of forms (i.e., physical and chemical) which in concert may act synergistically, additively, or antagonistically. Their individual accumulation in and effect on critical organs must be considered. Risk decreases with the decay of shorter and intermediate half-lived materials, but remains relatively constant for the long-lived radionuclides with half-lives in the thousands or millions of years. In some cases their daughter products may be of more significance than the parent nuclide. 


\section{RESPONSE}

The Preliminary Risk Assessment Report will consider the effects noted in the comment.

\section{COMMENT 23:}

Extensive referencing to several more manuals of field procedures has been utilized throughout the RI Plan, including the Field Sampling Plan (FSP) and Quality Assurance Project Plan (QAPP). The RI Plan should not simply reference these documents, but rather give specific details of how these documents will be implemented during the $R I$.

\section{RESPONSE}

A Field Sampling Plan has been prepared specifically for WAG 1. However, the many documents referenced by the RI Plan (such as the Project Management Plan, Waste Management Plan, ES\&H Plan, Quality Assurance Plan, and Project Procedures Manual) are generic to the multi-WAG investigations being conducted at ORNL. Field work guides are prepared for specific sampling campaigns that integrate the requirements of various project plans and procedures.

\section{COMMENT 24:}

The following conforming changes should be made throughout the RI Plan:

- "Feasibility Study" for "Alternative Assessment", and

- "Preliminary Risk Assessment" for "Preliminary Baseline Health Assessment".

\section{RESPONSE}

Agree. 


\section{RESPONSE TO CERCLA COMMENTS \\ ON WAG 1 RI PLAN}

\section{SPECIFIC COMMENTS}

\section{COMMENT 1}

Page iii, Table of Contents, Line 2.4: Delete "and Schedule" since schedule is not discussed in Section 2.4, but only in Section 2.5.

\section{RESPONSE}

This typographical error has been corrected.

\section{COMMENT 2}

Page 1-3, Para. 2, Line 14: Change "may address" to "will address".

\section{RESPONSE}

Individual SWMUs and groups of SWMUs will be addressed in the groundwater investigation. The investigation will not be limited by the data obtained from the WAG 1 perimeter monitoring wells, as implied by the original text.

\section{COMMENT 3:}

Page 1-3, Para. 3, Section 1.1.2.2: WAG-wide RI/FS activities are inadequate. Upon completion of the proposed RI Phase I work, and based on a Preliminary Characterization Summary Report and a Preliminary Risk Assessment Report, operable unit-specific RI Phase II Work Plans should be developed. The RI Phase II Work Plans should be developed with the flexibility to incorporate any additional sampling that may become necessary to facilitate efficient operable unit characterization.

\section{RESPONSE}

Phase II Work Plans, if required, will be developed to reflect this approach.

\section{COMMENT 4:}

Page 1-4, Para. 2, Section 1.2.1, Line 1: Delete the word "actual" before the term "RI". 


\section{RESPONSE}

The comment has been incorporated.

\section{COMMENT 5:}

Page 1-4, Bullet Item 3: Should read as follows: "Perform and document a risk assessment, including human exposure and environmental impact assessments, to define and prioritize contaminant sources within WAG-l requiring remedial action."

\section{RESPONSE}

Agree.

\section{COMMENT 6:}

Page 1-7, Para. 2: Add biota (flora and fauna) to the bulleted types of data to be collected. The RI Plan should document more specifically existing bioassay data, and explain how this information will be incorporated into the $R I$.

\section{RESPONSE}

The ongoing Biological Monitoring and Abatement Program (BMAP) conducted at ORNL presents its findings in published reports. These reports will be utilized in preparing the environmental characterization component of the risk assessment.

\section{COMMENT 7:}

Page 1-7, 3rd bullet: The RI Plan should also consider seismic refraction and resistivity surveys to define subsurface characteristics during RI Phase I field activities. These geophysical methods are effective in delineating fractured bedrock surfaces and vertical fracture zones.

\section{RESPONSE}

Normally, the use of seismic refraction and resistivity surveys could aid in defining fractured bedrock and karst trends. The use and effectiveness of these methods in WAG 1, however, are severely limited by site-specific features. Although seismic refraction can be used effectively to locate fractures via differences in bedrock velocity, buildings, interferences caused by pipelines and trenches, and vibrations due to plant operations and traffic would preclude its use in WAG 1. 
Similarly, the use of resistivity surveys would be of limited value in WAG .. To investigate a bedrock depth of $3.0 \mathrm{~m}(10 \mathrm{ft})$, electrodes must be placed at a distance of approximately $30 \mathrm{~m}$ $(100 \mathrm{ft})$. Congestion within the Main Plant Area would hinder use of resistivity surveys. Even outlying areas are not suitably free of man-made obstacles to render the method effective.

\section{COMMENT 8:}

Page 1-7 5th bullet: The RI Plan should also consider unsaturated zone sampling for volatile constituents.

\section{RESPONSE}

Target Compound List (TCL) volatiles are being collected on unsaturated soil samples.

\section{COMMENT 9:}

Page 2-1, Para. 3 and 5: The delineation of responsibilities for field activities between the WAG Manager and the FSS Manager is unclear. Figure 2-2, Page 2-3 shows these managers in parallel lines while Fig. 2-3, Page 2-6 shows the FSS Manager in a direct line under the WAG Manager.

\section{RESPONSE}

The current ORNL RI/FS organization, as shown in Attachment A, Response to CERCLA Specific Comment 9, supersedes Figures 2-2 and 2-3. The FSS manager supports the WAG Project Manager through a matrix organization.

\section{COMMENT 10:}

Page 2-1: Need to add description of the RI/FS Subcontract Project Manager's responsibilities before the WAG Manager's responsibilities to indicate communication between the WAG Manager and the FSS Manager for scheduling of field activities.

\section{RESPONSE}

The RI/FS Manager title no longer exists. This role is now filled by the Technical Integration Manager (TIM) as shown in the organization chart addressed in the Response to CERCLA Specific Comment 9. The TIM also supports the WAG Project Manager through a matrix organization. 
COMMENT 11:

Page 2-3, Figure 2-2: Need to change "RI/FS Manager" block to "RI/FS Subcontract Project Manager" to be consistent with Figure 2-1, Page 2-2.

\section{RESPONSE}

Please refer to the Response to CERCLA Specific Comment 9. The RI/FS Subcontract Project Manager position no longer exists. The duties of the RI/FS Manager are now performed by the TIM.

\section{COMMENT 12:}

Page 2-4, Para. 4, Lines 1 and 2: The FHS Supervisor is responsible to whom? Is it the Environmental Safety \& Health (ES\&H) Manager or the FSS Manager?

\section{RESPONSE}

The FHS is directly responsible to the EH\&S Manager for the project.

COMMENT 13:

Page 2-4, Para. 5: Where does the Review Team Leader fit into the organization shown in Figure 2-2 (Page 2-3)?

\section{RESPONSE}

The Review Team Leader is a senior manager whose involvement in the project is coordinated by the TIM.

\section{COMMENT 14:}

Page 2-7, Para. 3, Line 5: QAPPs should be specified here (i.e., Quality Assurance Project Plans).

\section{RESPONSE}

Agree. 
COMMENT 15:

Page 2-11, Section 2.4, Heading: Delete "And Schedule" since schedule is not discussed in this section.

RESPONSE

Agree.

COMMENT 16:

Page 2-11, Section 2.4, Para. 1, Lines 3 and 4: Delete "and presents the preliminary schedule for the $R I^{\prime \prime}$.

RESPONSE

Agree.

COMMENT 17:

Page 2-12, Figure 2-6: Needs provision for a community relations task. Also include "air" and "biota" under 201300. Also, include the following:

- Preliminary Risk Analysis in 201500,

- Preliminary Characterization Summary Report in 201600, and

- Community Relations Activities in 201200.

\section{RESPONSE}

Air and biota are included under 201300 in the work breakdown structure as shown in Attachment A, Response to CERCLA Specific Comment 17.

Preliminary risk analysis is shown in 201500 , and a Site Characterization Summary Report in 2015CS.

Community relations activities are not included in the work breakdown structure as they are not included as part of subcontractor RI/FS activities. DOE maintains a community relations program for the Oak Ridge Reservation. 


\section{COMMENT 18:}

Page 2-13, Figure 2-7: Why are analytical services and data validation under ES\&H Manager? Validation should be done by the RI/FS Subcontract Manager to conform with Figure 2-1 and Figure 2-2.

\section{RESPONSE}

Since Revision 1 was written, both analytical services and data validation functions are the responsibility of the TIM. See the figure attached to Response to CERCLA Specific Comment 9 for the RI/FS Project Organization Chart.

\section{COMMENT 19:}

Page 2-14, Para. 3, Line 5: Why are Quality Assurance Assessments (QAAs) being performed by the WAG Manager and not by the Quality Assurance/Quality Control Manager (see Figure 2-2, Page 2-3)? If the WAG Manager conducts the QAA, will the WAG Manager incorporate the results of the RTL reviews and the $Q A$ Audit Team reviews?

\section{RESPONSE}

Quality Assurance Assessments (QAAs) will be performed by the QA manager as specified by the QA Plan for the ORNL RI/FS (BNI 1988). Periodic quality reviews of project plans, ongoing project activities, project files, and project deliverables are conducted by the Review Team. Field inspections are conducted by QC supervisors on a routine basis.

\section{COMMENT 20:}

Page 2-15, Para. 3: What about the use of surface geophysical surveys to locate waste disposal trenches, and down-hole geophysical surveys in boreholes and wells? The RI Plan should consider seismic refraction and resistivity geophysical methods.

\section{RESPONSE}

The use of surface geophysical surveys (seismic refraction and resistivity surveys) in WAG 1 is limited by site-specific features such as buildings, pipeline trenches, and vibrations due to plant operations and traffic. See Response to CERCLA Specific Comment 7 for a more detailed discussion. However, downhole geophysical surveys will be performed in approximately 11 deep bedrock coreholes. These coreholes are located along three north-south transects, and will be completed to depths up to $152.4 \mathrm{~m}(500 \mathrm{ft})$. 


\section{COMMENT 21:}

Page 2-15, Para. 3, Numbered Item 3: Consider Ground Penetrating Radar to correlate with EM-31 survey.

\section{RESPONSE}

Ground penetrating radar (GPR) is not appropriate for use in WAG 1. GPR does not perform well in soils having a high clay content, as found in soils overlying the Chickamauga Group. GPR was previously attempted in settings similar to those present in WAG 1 (Geo-Centers, Inc. 1982). ORNL concluded that its use was ineffective.

\section{COMMENT 22:}

Page 2-16, Para 4, Line 3: Add biota to media sampling list.

\section{RESPONSE}

Please see Response to CERCLA Specific Comment 6 for a more detailed discussion.

\section{COMMENT 23:}

Page 2-17: Existing data should be analyzed during the RI Phase I effort to insure that all applicable data and information can be incorporated into operable unit - specific RI Phase II Work Plans.

\section{RESPONSE}

The Existing Data Analysis task already present in this plan will facilitate the evaluation of any additional existing data that become available during the RI. The Phase II Work Plans will be operable unit-specific.

\section{COMMENT 24:}

Page 2-19, Para. 3: Technical Interim Memoranda should be submitted quarterly.

\section{RESPONSE}

Technical memorandums are an appendix to the Site Characterization Summary Report and the RI Report. Technical memorandums are issued at the completion of major tasks such as groundwater investigations. 


\section{COMMENT 25:}

Page 2-19, Para. 4, WBS 201700: Title should be changed from "Lab and Bench-Scale Studies" to "Lab, Bench-Scale, and Pilot Treatability Studies". These studies should be integrated with RI Phase II activities, with data needs identified as part of RI Phase I work.

\section{RESPONSE}

Please refer to the Response to CERCLA General Comment 14 for further information.

\section{COMMENT 26:}

Page 2-19, Section 2.5: This section should document the specific timelines for events scheduled during RI Phase I activities and the general timelines for RI Phase II activities. The ORR FFA negotiated schedule could then effectuate the timelines which should be shown in Figure 2-8.

\section{RESPONSE}

The attached figure (Attachment A, Response to CERCLA Specific Comment 26) shows the specific timelines for Phase I activities. Phase II timelines will be negotiated in the manner specified in the proposed FFA.

\section{COMMENT 27:}

Page 2-20, Figure 2-8: The schedule needs updating and specification. It also needs to show more details such as the integration of the Energy Systems source testing into the RI, and periods for regulatory review and public comment. The schedule should include a breakdown of timelines or periods of duration concerning the following phases of work:

- project scoping including a complete evaluation of existing data and information,

- screening investigations, and RI Phase I sampling and analysis,

- Preliminary Characterization Summary Reports and Preliminary Risk Analysis Reports,

- RI Phase II sampling and analysis, and any additional sampling,

- Treatability Studies,

- Baseline Risk Assessment Reports,

- RI and FS Reports,

- Proposed Plans, and

- Records of Decision. 


\section{RESPONSE}

The schedule is updated periodically in accordance with the proposed FFA, and in response to other agreements between EPA and DOE. DOE funding allocations also influence periodic schedule adjustments.

\section{COMMENT 28:}

Page 2-21, Para. 4: A report should be made documenting the purging of rejected data.

\section{RESPONSE}

The Data Quality Review Procedures section discusses the process of data validation and qualifiers. For example, the R-qualified data are indexed for "limited use" but are not purged from the project files. No data are ever purged from the project files but are kept in data files for future reference.

\section{COMMENT 29:}

Page 2-22, Section 2.7, Lines 5-10: The WAG 1 RI Plan should specifically document the implementation of the generic ES\&H Plan. This documentation should include any specific health and safety concerns, and explain how these concerns will be considered during the RI activities.

\section{RESPONSE}

The Project-wide ES\&H Plan serves as the basis for developing sampling event-specific ES\&H protection measures. These measures are documented in Field Work Guides prepared for each event.

\section{COMMENT 30:}

Page 3-1, Section 3.1: The descriptions of the environmental setting should be consolidated for WAG 1 - specific information as well as site-wide and regional information rather than splitting off WAG 1-specific information to Section 3.3. The Biota discussion, Section 3.1.6.8, is more appropriate.

\section{RESPONSE}

DOE believes it is necessary to understand the broader regional environmental framework before assessing how an individual WAG functions within that framework. Therefore, the current format of presentation is retained. 
COMMENT 31:

Page 3-2, Figure 3-1: Need additional map with reduced scale to define WAG-1 within ORNL.

\section{RESPONSE}

The map included as Attachment A, Response to CERCLA Specific Comment 68 indicates the boundary of WAG 1 within the ORNL area.

\section{COMMENT 32:}

Page 3-3, Para. 1, Lines 4-6: The reservation water bodies serve as flyways for migrating water fowl, and as source water sustaining other animals such as deer. Also of concern, is the wetland vegetation that may be consumed.

\section{RESPONSE}

Agree.

\section{COMMENT 33:}

Page 3-4, Para. 1, Line 8: What current waste management practices may be of environmental concern for the "minority of problems"?

\section{RESPONSE}

Present waste management practices at ORNL are considered to be state of the art. It is not anticipated that these practices are of "environmental concern."

\section{COMMENT 34:}

Page 3-5, Para. 2: A topographic map is necessary to illustrate the text.

\section{RESPONSE}

See Attachment A, Response to CERCLA Specific Comment 64 for a topographic map of the area. 
COMMENT 35:

Page 3-7, Figure 3-2: This figure depicts a stratigraphic column, not a geologic crosssection.

\section{RESPONSE}

Agree.

\section{COMMENT 36:}

Page 3-9, Para. 1: The statement that "it is improbable that a shock of major intensity will occur in the Oak Ridge area for several thousand years" seems to conflict with the statement in the previous paragraph that there is a 10 percent probability that a seismic event... would cause minor damage to structures at Oak Ridge.

\section{RESPONSE}

There is not conflict between the two statements. The 10 percent probability of a seismic event refers to a minor to moderate potential level of damage to structures. An earthquake of greater intensity has a lower probability of occurrence.

\section{COMMENT 37:}

Pages 3-10 to 3-12, Section 3.1.6.4: Available historical surface water quality data, especially with respect to radiological isotopes $\mathrm{Co}, \mathrm{Cs}, \mathrm{Sr}$, etc., should be presented here.

\section{RESPONSE}

Attachment A, Response to CERCLA Specific Comment 37 outlines radionuclide and alpha emitter water quality data taken in 1985 from selected collection stations. Nine public water supply systems serve about 91,500 people within a $32-\mathrm{km}(20$-mile) radius of the ORR. One of these nine supply systems (City of Kingston) is downstream of the ORR. The intake for Kingston is located on the Tennessee River at about 0.6 river $\mathrm{km}(0.37 \mathrm{miles})$ above the confluence of the Clinch and Tennessee Rivers and about 34.1 river $\mathrm{km}$ ( 21.3 miles) below the White Oak Dam outfall. Kingston withdraws approximately 9 percent of its average daily supply from the Tennessee River. The most up-to-date data will be incorporated in the RI Report. 


\section{COMMENT 38:}

Page 3-12, Para. 1: Need to expand the discussion on the effect that ORR activities have on the Clinch River, specifically how WAG-l contributes to contamination of the Clinch River.

\section{RESPONSE}

All drainage and effluents from WAG 1, as well as from other WAGs, discharge to White Oak Creek and therefore, to White Oak Lake. Under high flow conditions both waterborne radioactivity and radioactively contaminated sediments can be carried through White Oak Dam and discharged to the Clinch River. WAG 1 has been a historic contributor of ${ }^{90} \mathrm{Sr}$ and ${ }^{137} \mathrm{Cs}$ to the overall contaminant inventory.

\section{COMMENT 39:}

Page 3-12: Document the potential targets (i.e., population of concern) for the drinking water pathway for surface water.

\section{RESPONSE}

All water used on the ORR is imported from the Clinch River. Potential receptors for surface water today include only biota. Potential future receptors are homesteaders who may settle on the ORR after the institutional control period. The potential off-site receptor is a homesteader located on the Clinch River below the White Oak Dam outlet.

\section{COMMENT 40:}

Page 3-13: Document the potential targets for the drinking water pathway for groundwater.

\section{RESPONSE}

Sixteen public groundwater supplies are located within a $32-\mathrm{km}$ (20-mile) radius of ORNL. Of these sources, the closest to ORNL is the Allen Fine Spring supplying the Dixie-Lee Utility District in Loudon County. This groundwater source, about $10.9 \mathrm{~km} \mathrm{(3.3} \mathrm{miles)}$ southeast of ORNL, serves 6700 people with an average of $1500 \mathrm{~m}^{3}\left(52,966.1 \mathrm{ft}^{3}\right)$ of water per day.

Private single-family wells are common in adjacent rural areas not served by public water supply. Most of the residential wells, other than those adjacent to the City of Oak Ridge, are south of the Clinch River. The nearest resident is approximately $3.7 \mathrm{~km}$ ( 2 miles) from the WAG 1 boundary. No residential wells would be located closer. 


\section{COMMENT 41:}

Page 3-13: Specify the WAG-l groundwater flow regime.

\section{RESPONSE}

This section of the RI Plan addresses the regional environmental characteristics of the Oak Ridge area. Discussion of the WAG 1 flow regime is provided in Section 3.3.1.4 of the WAG 1 RI Plan, Revision 1 (August 1989). Responses to CERCLA Specific Comments 62 and 98 provide additional information on the WAG 1-specific flow regime.

\section{COMMENT 42:}

Pages 3-15 to 3-25, Section 3.1.6.8: The effects on biota is of environmental concern. Also, biota could serve as a pathway for human exposure and should be a part of the RI risk assessments.

\section{RESPONSE}

DOE agrees that biota serve as potential pathways for human exposure. The ongoing Biological Monitoring and Abatement Program conducted at ORNL presents its findings in published studies. These reports will be utilized in preparing the environmental characterization of the risk assessment.

\section{COMMENT 43:}

Page 3-22, Table 3-6: How significant are these differences between upstream and downstream occurrences, and can they be attributed to radiological contamination of the White Oak basin emanating from the ORNL?

\section{RESPONSE}

The significance of these differences will be determined as part of the WAG 2 White Oak Lake RI activities.

\section{COMMENT 44:}

Page 3-23, Para. 1, Lines 1 and 2: This monitoring program should be evaluated during the $R I$ for adequacy of its coverage of ORNL WAG-l sampling, analytical and $Q A / Q C$ procedures to ensure that the data can be incorporated into the RI. 


\section{RESPONSE}

Agree.

\section{COMMENT 45:}

Page 3-23, Para. 1: Need to present the vegetative analysis results.

\section{RESPONSE}

Grass samples were collected at ORNL perimeter stations and ORR stations. (See Attachment A, Response to CERCLA Specific Comment 45 for location map). These samples were analyzed for several radioactive isotopes. Table 1 in Attachment A, Response to CERCLA Specific Comment 45 summarizes the results of these analyses. Table 2 presents individual analyses of the ORNL perimeter samples. The grass samples at Station 4 , which is very close to the Process Waste Treatment Plant in WAG 1 , had concentrations of ${ }^{137} \mathrm{Cs}$ and total radioactive $\mathrm{Sr}$ that were about two orders of magnitude greater than typical values of other stations. Concentrations of ${ }^{239} \mathrm{Pu}$ were also anomalously high in grass around Station 4. These observed concentrations in samples at Station 4 may be due to overflows from impoundments (MMES 1989).

\section{COMMENT 46:}

Page 3-23, Para. 1, Last sentence: What is the significance of these results? A discussion would be useful.

\section{RESPONSE}

The significance of these results will be determined as part of the WAG 2 White Oak Lake RI and the Clinch River RFI.

\section{COMMENT 47:}

Page 3-23, Para. 4: What was the diet of the deer with Sr-90 reported in bone? Where did they graze? What was source of the water intake? Was the primary source of Sr-90 from plant and water intake, or soil exposure?

\section{RESPONSE}

Interviews with ORNL researchers revealed that stomach contents of the deer have not been analyzed. The deer were not radio-collared and, therefore, the deer's specific movements are unknown. Analyses have been performed on vegetation associated with seeps downgradient of 
burial grounds in Melton Valley. The analyses suggest that the ${ }^{90} \mathrm{Sr}$ may have been derived from honeysuckle and blackberry shoots growing in the seep areas. The model used to calculate exposure time indicated that a $45 \mathrm{~kg}$ buck could acquire greater than $30 \mathrm{pCi} / \mathrm{g}{ }^{90} \mathrm{Sr}$ in its bone after eating the vegetation during a period ranging from 1 week to 1 year.

\section{COMMENT 48:}

Page 3-25, Table 3-8: Are the results reported on the basis of wet or dry weight? The concentration of radioactivity in the bone of geese seems high. Verify the unit of measurement is $\mathrm{pCi} / \mathrm{g}$ and not pCi/kg. Sampling of biota tissue should be a part of the RI.

\section{RESPONSE}

Results were reported on the basis of wet weight. Units of measurements, $\mathrm{pCi} / \mathrm{g}$, are correct. Biota monitoring is included in the Biological Monitoring and Abatement Program. The latest available information will be reported in the environmental evaluation.

\section{COMMENT 49:}

Page 3-28, Section 3.2: The nature and extent of contamination discussion is too general. Need to document specific information on media types contaminated in WAG-1. For example, considerable information exists concerning known or suspected contaminants from tanks, leak and spill sites, and waste treatment facilities, and yet is not well documented by the RI Plan.

\section{RESPONSE}

Attachment A, Response to CERCLA Specific Comment 49 is a table that lists each SWMU, the contaminant(s), and inventory where known.

Media-specific contamination is discussed in detail in Section 3.3 of the WAG 1 RI Plan, Revision 1. Tables that exist in the RI Plan outlining media-specific data include:

Table Data

3-11a Ranges of radionuclides-soil

3-12 Radiological characteristics of each tank site

3-12a Ranges of $\mathrm{Hg}$ concentrations-soil

3-14 Radionuclide concentrations-water in White Oak Creek

3-15 Radionuclide concentrations-stream sediment

3-15a Ranges of $\mathrm{Hg}$ concentrations-stream sediment

3-15b Ranges of radionuclides-sediment 7500 Bridge 
Hg concentration-sediment parameters-Piezometer scoping survey

\section{COMMENT 50:}

Page 3-29: Figure 3-6 needs a scale.

\section{RESPONSE}

Agree.

\section{COMMENT 51:}

Page 3-32, Para. 1 and 2: How were the wastes disposed of? What type of wastes were they specifically? What happened to sludge or materials removed in treatment? At what levels were wastes allowed to be released into the Waste Water Treatment System.

\section{RESPONSE}

The RI Plan is supplemented with the following text:

When ORNL was built, an extensive underground piping system was installed to collect intermediate-level waste (ILW) and transport it to large underground concrete ("gunite") tanks designed for permanent storage (Sears, 1987). Eleven gunite tanks, W-1 to W-11, were installed in 1943 . W-1 to W-4 received wastes from radiochemical processing facilities, W-11 received wastes from research laboratories, and W-5 to W-10 originally received waste from the graphite reactor. These tanks, W-5 to W-10, were subsequently used as part of the intermediate level liquid waste system until 1980 when new tanks were brought on line. Three small steel tanks, W1A, WC-15, and WC-17, were installed in 1952 to store research laboratory wastes. These tanks were removed from service and emptied due to leakage. Gunite tank TH-4, installed in 1943, collected waste from pilot plant projects and was removed from service in 1970 . This tank is full and is not known to be leaking. In addition, buildings at ORNL generating radioactive wastes were provided with waste collection tanks. Wastes were stored and generally sampled before a decision was made regarding disposition of the waste (i.e., storage in the main tanks or release to the process waste system for treatment before disposal).

Waste in the tanks was treated with caustic to precipitate radionuclides, and the resulting sludges stored in the tanks. Originally the supernatant liquid was decanted, diluted, and released to White Oak Creek at an unknown activity level. In 1944 a settling basin was completed to allow additional time for gravity settling of radioactive solids before discharge of 
supernatant liquids. Release of supernatant directly to WOC was discontinued in 1949. From June 1949 to June 1954, the liquid waste was concentrated in an evaporator, with the condensate discharged to WOC. The concentrate was stored in the gunite tanks to allow solids to settle. From 1952 to 1957 the contents from these tanks were mined for fissile material. Thereafter, they were used for waste management in conjunction with operations of pits and trenches (WAG 7) and the hydrofracture facility (WAG 10). Concerns about groundwater contamination led to discontinuation of seepage disposal in 1966. Disposal of liquid waste concentrate by the hydrofracture process was inaugurated in 1966 and continued until 1985. Currently, the evaporator concentrate is being stored pending decision on permanent disposal. According to ORNL personnel, most of the sludge was sluiced out of the gunite tanks in the early 1980's. Some of the sludge was sent to the hydrofracture facility and a portion of the sludges are now in the Melton Valley storage tanks. "Unsluicable" solids remain in the gunite tanks.

Major radionuclides routinely handled by the system include ${ }^{90} \mathrm{Sr},{ }^{137} \mathrm{Cs}$, and ${ }^{60} \mathrm{Co}$, with some $\mathrm{Pu}, \mathrm{U}$, and TRU isotopes present in the waste stream. Most sources generated dilute low level waste at the $\mathrm{mCi} / \mathrm{L}$ activity level. Occasional wastes up to $76 \mathrm{Ci} / \mathrm{L}$ were diluted to $0.2 \mathrm{Ci} / \mathrm{L}$ before storage in cullection tanks.

None of the liquids in the tanks contain significant TRU or other radioactivity beyond lowlevel waste limits. However, the sludge in tanks W-3, W-4, and W-7 to W-10 is classified as TRU waste. The sludge in other tanks contains TRU below the $100 \mathrm{nCi} / \mathrm{g}$ limit for TRU waste. Since much of the waste has already been identified as TRU waste, the assumption is that all sludge is TRU waste.

All of the sludges and most of the liquids contain metals $(\mathrm{Cr}, \mathrm{Pb}$, and $\mathrm{Hg})$ above the $\mathrm{EP}$ toxicity levels. Nine of the tanks are known to contain EPA-listed organics, but only W-7 and WC-17 have organics above regulatory Toxicity Characteristic Leaching Procedures (TCLP) toxicity levels as indicated by the present samples. Because of the uncertainty in the representativeness of the samples, other tanks may contain organics above TCLP toxicity levels.

Documentation of tank contents is ongoing. Extensive data on the contents of ORNL tanks can be found in Autrey et. al. (1990a and b). These documents will be provided under separate cover.

\section{COMMENT 52:}

Page 3-32, Para. 3: What is meant by tanks "removed from service?" Were the tanks and contents disposed of, and how? 


\section{RESPONSE}

The phrase "removed from service" means that the tanks ceased to receive programmatic waste (i.e., become inactive).

In general, inactive tanks remain in place and contain liquid wastes and/or sludges contaminated with radionuclides and possibly hazardous chemicals. An assessment of the disposition of the wastes in these tanks is currently in progress.

\section{COMMENT 53:}

Pages 3-33 to 3-42, Table 10: Does N/A stand for not available, or not applicable? Need to specify.

\section{RESPONSE}

In the table N/A means not applicable.

\section{COMMENT 54:}

Page 3-34, Table 3-10: The last contaminant of SWMU 151 should be Ce-141.

\section{RESPONSE}

Agree.

\section{COMMENT 55:}

Page 3-42, Table 3-10: Item 1.53 Bldg. 3000 Septic Tank. Of concern would be the tile field or more so than tank unless overflowing. (sic)

RESPONSE

Agree.

COMMENT 56:

Page 3-48, Para. 4, Lines 4 and 5: What was done about the contaminated storm drain? 


\section{RESPONSE}

Response actions to this spill site are not documented.

COMMENT 57:

Page 3-49, Para. 3 and 4: How and where was contaminated soil disposed?

\section{RESPONSE}

The manner of disposal of the contaminated soil is unknown.

COMMENT 58:

Page 3-51, Last two lines on page: Typographical errors: cadmium-115, and cerium-141.

\section{RESPONSE}

Agree.

COMMENT 59:

Page 3-55, Para. 2, Line 5: cadmium-244 should be curium-244.

\section{RESPONSE}

Agree.

COMMENT 60:

Page 3-58, Section 3.2.3: The ponds and impoundments discussion needs specification concerning potential contaminants in waste streams.

\section{RESPONSE}

Additional information on known/potential contaminants in ponds and impoundments waste streams has been incorporated, to the extent known, in Section 3.2.3. 


\section{COMMENT 61:}

Page 3-62, Para. 1: Need to specify radionuclide contamination.

Page 3-62a, Section 3.2.6: Need to provide the meaning of the phrase "contaminant source term data". Also, more specific information concerning potential contamination should be documented here.

\section{RESPONSE}

Analyses of both a canal and dissolver pit liquid and solid samples indicate the presence of ${ }^{239} \mathrm{Pu},{ }^{241} \mathrm{Am},{ }^{235} \mathrm{U},{ }^{238} \mathrm{U},{ }^{137} \mathrm{Cs}$, and ${ }^{90} \mathrm{Sr}$. The analysis results are shown in Attachment $\mathrm{A}$, Response to CERCLA Specific Comment 61.

The contaminant source term data are the known and suspected chemicals and radionuclides that are present in various media for SWMUs. Please refer to the Response to CERCLA Specific Comment 51 for further inforination on potential contamination.

\section{COMMENT 62:}

Page 3-63, Section 3.3.1: All subsections, 3.3.1.1, 3.3.1.2, 3.3.1.3, 3.3.1.4 and 3.3.1.5 should include discussions on how WAG-l specific media concerns will potentially affect the implementation of the FSP.

\section{RESPONSE}

The following text supplements the original text:

Geology. Solution cavities and channels in the Chickamauga Group may present potential problems during drilling activities since loss of drilling fluids and grout occur.

Soils. Soils in WAG 1 have been highly disturbed by construction activities. Reworked native soil and imported fill have been placed in pipeline trenches, under foundations, as backfill around buildings, and in other excavations throughout the site. As a result of varying fill material, a range of soil characteristics, including permeability, porosity, compaction, and composition, will be encountered. Backfilled areas such as pipeline trenches could provide preferred pathways for contaminant migration.

Surface Water and Sediments. Potential problems concerning surface waters and FSP implementation include flooding and drought. Flooding along WAG 1 drainages may damage flow monitoring devices if they are not properly designed and constructed. Periods of prolonged drought would prevent the characterization of high flow conditions. 
Groundwater. The interconnectedness of the pipeline trenches will affect the sampling strategy in several ways. It will be difficult to determine the location and nature of a single source of contamination at a particular location on the site. It may be necessary to infer a hypothetical source through modeling to provide necessary risk assessment parameters. Because of the large variation in hydraulic conductivity between pipeline trenches and the surrounding less disturbed soil, contaminant plumes may be expected to follow pipeline trenches. Sampling that ignores this preferential path may present an inaccurate picture of actual contamina:i plumes. Developing an accurate picture of site contamination may require relying more heavily on biased sampling that focuses on trenches rather than on unbiased sampling.

Local groundwater flow patterns at ORNL are significantly affected by man-made features, including active sump pumps, directional permeabilities, and local recharge from impoundments. Water levels in nearby wells and piezometers will also be affected by these activities. Slow recharge rates may impede well development and sampling efforts. Groundwater flow through jointed and fractured bedrock, through interconnected solution cavities and channels, and along bedding planes may prove difficult to predict and model.

Air. No potential problems or specific media concerns for air that sould affect FSP implementation have been identified.

\section{COMMENT 63:}

Page 3-63, Para. 3: What other types of 'own-hole geophysical data (e.g., susceptibility, density, resistivity or conductivity) was obtained? All data could be useful in correlating surface geophysical models from seismic, resistivity, Ground Penetrating Radar and electromagnetic survey data.

\section{RESPONSE}

The geophysical log suite run in these coreholes included gamma, caliper, spontaneous potential, compensated density, neutron porosity, single point resistivity, long/short normal resistivity, temperature, and borehole deviation. Data will be used in correlating subsurface geophysical models.

\section{COMMENT 64:}

Page 3-67, Figure 3-8: This figure needs a scale, WAG-l should be depicted and the clarity of the figure should be improved. 


\section{RESPONSE}

A revised geologic map is shown in Attachment A, Response to CERCLA Specific Comment 64.

\section{COMMENT 65:}

Page 3-70, Table 3-12: Note that the depths of maximum concentrations of the various cores vary from $0.3 \mathrm{~m}(\mathrm{ft})$ to $0.6 \mathrm{~m}$ (2ft). These hot spots would be diluted by compositing 5-fi samples.

\section{RESPONSE}

Field screening methods to isolate specific horizons for analysis are being employed.

Compositing does not exceed $0.6-\mathrm{m}$ (2-ft) intervals.

\section{COMMENT 66:}

Page 3-74b, Figure 3-10: WAG-1 should be depicted.

\section{RESPONSE}

New figure is shown in Attachment A, Response to CERCLA Specific Comment 66.

\section{COMMENT 67:}

Page 3-75, Para. 2, Lines 1 and 2: Referenced Figure A7-1 is not included in RI Plan.

\section{RESPONSE}

Reference should be made to the map in Attachment A, Response to CERCLA Specific Comment 68 for the approximate location of the critical flow meter station.

COMMENT 68:

Page 3-76, Figure 3-11: This figure needs a scale, and WAG-1 should be depicted.

\section{RESPONSE}

A revised figure is shown in Attachment A, Response to CERCLA Specific Comment 68. 
COMMENT 69:

Pages 3-78 to 3-80: Figures 3-12, 3-13 and 3-14 all need a scale.

\section{RESPONSE}

Agree.

COMMENT 70:

Page 3-82: Table 3-13 should also document recent analysis results.

\section{RESPONSE}

A table that documents the most recent published data is provided in Attachment A, Response to CERCLA Specific Comment 70.

\section{COMMENT 71:}

Page 3-83, Table 3-14: The table indicates that tritium concentrations are elevated. The RI Plan should provide for analysis for all known or suspected radionuclides, include a separate sampling method for analyzing volatile radioactive isotopes such as tritium, carbon14 etc.

\section{RESPONSE}

Analyses of all known or suspected radionuclides are being performed. Appropriate preservatives and holding times for volatile radionuclides are being observed (see Response to CERCLA Specific Comment 184). Generally, the radionuclides being analyzed are determined in accordance with the radiological analytical strategy specified in Section A10.2.1 of the FSP. Based on known or suspected contaminants at a particular location, analysis of specific radionuclides is requested. Tritium is routinely analyzed.

The radiological analytical strategy is presented in Section A10.2.1 of the FSP. As indicated in Attachment A-3 of the FSP, samples undergo initial gross alpha/beta scan and, depending on the results, are submitted for further analyses using gamma spectroscopy, alpha spectroscopy, or beta emitter isotopic by liquid scintillation. 


\section{COMMENT 72:}

Page 3-86a, Figure 3-16A: This figure needs a scale.

\section{RESPONSE}

Agree.

\section{COMMENT 73:}

Page 3-86c, Figure 3-16B: What is the unit of measurement for $\mathrm{Hg}$ concentrations.

\section{RESPONSE}

The unit of measurement for $\mathrm{Hg}$ concentrations is $\mathrm{Mg} / \mathrm{g}$.

\section{COMMENT 74:}

Page 3-86d, Para. 2: What were the levels of referenced radionuclide contamination.

\section{RESPONSE}

As reported by Cutshall (1985), the contaminant level is several orders of magnitude lower than that typically measured in lower White Oak Creek, downstream of WAG 1. For a discussion of Cutshall's findings, refer to Response to CERCLA Specific Comment 94.

\section{COMMENT 75:}

Page 3-86d, Para. 3 and Table 3-15: $\mathrm{Hg}$ resuits are expressed as ppm. Were the sediment samples analyzed for total $\mathrm{Hg}(\mathrm{mg} / \mathrm{kg})$ or were EP Toxicity extracts of sediment samples analyzed $(\mathrm{mg} / \mathrm{l})$ ?

\section{RESPONSE}

Mercury levels for sediment samples collected from White Oak Creek have been reported for 1979 and 1983. Analyses associated with WAG 1 are shown in Attachment A, Response to CERCLA Specific Comment 75. The highest value recorded in 1979 was 3.8 ppm at site $\mathrm{T}-10$, which is located just downstream of the Equalization Basin and the Process Waste Settling Basin. Samples collected in 1983 from a similar location had mercury levels at 18 and $19 \mathrm{ppm}$. 
The White Oak Creek sediment samples were not analyzed for EP toxicity. The samples were analyzed for total concentration.

\section{COMMENT 76:}

Page 3-90, Figure 3-18: This figure needs a scale.

\section{RESPONSE}

Agree.

COMMENT 77:

Page 3-92, Para. 1: This discussion is in conflict with Para. 4, Page 3-92e.

\section{RESPONSE}

In general, WAG-wide groundwater contours appear to follow surface topography and flow is down dip (Ketelle et al., 1986). Localized studies, such as dye tracer tests, within portions of the WAG boundary have indicated that groundwater may also have an along-strike flow component (Melroy, 1986).

\section{COMMENT 78:}

Page 3-92, Para. 2: Was $\mathrm{Hg}$ not included in the scoping analysis?

\section{RESPONSE}

Mercury analyses for the scoping event were not reported.

\section{COMMENT 79:}

Page 3-92, Para. 3 and 4: The referenced data in Tables 3-17, 3-18 and 3-19 should be shown with available ARARs for comparison.

\section{RESPONSE}

ARARs are presented for the data contained in Table 3-17 (piezometer scoping survey) in Attachment A, Response to CERCLA Specific Comment 79. ARARs for the data contained in Tables 3-18 (Impoundment 3539-3540) and 3-19 (Impoundment 3524) are presented in Attachment A, Response to CERCLA Specific Comment 94. 
COMMENT 80:

Page 3-92a, Para. 1: The referenced studies and reports should be provided to EPA and TDHE as supporting documentation to the RI Plan.

\section{RESPONSE}

Referenced studies and reports will be made available.

\section{COMMENT 81:}

Page 3-92a, Para. 3, Line 4: Cooperations should be changed to operations.

\section{RESPONSE}

Agree.

\section{COMMENT 82:}

Page 3-92c, Line 16: It is also possible that contamination exfiltrates from the clay pipe into surrounding soils during low water table conditions. Then, during high water table conditions groundwater could become contaminated via desorption.

\section{RESPONSE}

This mechanism is possible; however, surveillance data from pumping station \#1 (which is where the tank farm drainage in the clay pipe collects) indicates there is always an influx of groundwater. Even in dry months, approximately 350,000 gal per month is collected. This lends credibility to the argument that the process is always infiltration rather than exfiltration.

COMMENT 83:

Page 3-92c, Para. 1: What type of contamination exists at Manhole 233?

\section{RESPONSE}

Water entering manhole 233 , if contaminated, is likely to contain liquid low level radioactive waste. 
CERCLA SPECIFIC COMMENTS

\section{COMMENT 84:}

Page 3-92d, Line 5: Discuss how the "interconnectedness" will affect the sampling strategy for RI Phase I sampling.

\section{RESPONSE}

The interconnection of pipeline trenches will affect the sampling strategy in several ways. Because of the large variation in hydraulic conductivity between pipeline trenches and the surrounding less disturbed soil, contaminant plumes may be expected to follow pipeline trenches. It will be difficult to determine the location and nature of a single source of contamination at a particular location on the site. Sampling that ignores this preferential pathway may present an inaccurate picture of actual contaminant plumes. Developing an accurate picture of site contamination may require relying more heavily on biased sampling that focuses on trenches rather than on unbiased sampling.

\section{COMMENT 85:}

Page 3-92d, Para. 2: The term "ICP" should be added to the acronym list, Page xi.

\section{RESPONSE}

"ICP" is now a part of the acronym list as "Inductively Coupled Plasma."

\section{COMMENT 86:}

Page 3-92e, Para. 2: The findings of Melroy (1986) are in conflict with the last sentence of this paragraph. Due to the fact that Melroy (1986) determined that the two main , components of groundwater flow are:

- along geologic strike, and

- to the southeast (i.e., down-dip);

it is possible that contaminants are migrating via fracture zone and bedding plane conduits where the pipeline trenches intersect with bedrock. Thus, contamination in the Main Plant Area may in fact exist at a depth interval below the pipeline trenches.

\section{RESPONSE}

While Ashwood (1988) suggests that trenches exert a significant influence on groundwater and contaminant movement, Melroy's (1986) study indicates deeper multidirectional migration paths. The conflict between these two studies reinforces the general belief that conditions governing groundwater flow are complex. Samples from existing wells screened in bedrock combined with samples from discrete intervais from deep coreholes wiil be used to 
characterize the general nature of groundwater quality in bedrock fractures during Phase I. Further investigation of groundwater quality in bedrock fractures will be considered in developing Phase II investigation plans.

COMMENT 87:

Page 3-92e, Para. 4: All available MMES (1989) sampling results should be documented by the RI Plan.

\section{RESPONSE}

All available sampling results were provided when the RI Plan, Revision 1, was issued. See Response to CERCLA General Comment 8 for the location of additional data included in this response package.

\section{COMMENT 88:}

Page 3-92f, Line 1: Specify the radionuclides that were selected for analysis. Also, need to summarize inorganic and organic constituent concentrations found.

\section{RESPONSE}

Analyses were performed for groundwater quality constituents listed in 40 CFR 265, gross alpha, gross beta, ${ }^{60} \mathrm{Co},{ }^{137} \mathrm{Cs}$, total radioactive $\mathrm{Sr}$, total $\mathrm{Ra}$, and ${ }^{3} \mathrm{H}$.

Well 812 had the highest gross alpha $(8.6 \mathrm{~Bq} / \mathrm{L})$, gross beta $(660 \mathrm{~Bq} / \mathrm{L})$, and radioactive $\mathrm{Sr}$ $(280 \mathrm{~Bq} / \mathrm{L})$. Parameters exceeding the National Primary Drinking Water Limits include fecal coliform $(3 \mathrm{COL} / 100 \mathrm{~mL}$ in wells 822 and 827$)$, fluoride $(3.8 \mathrm{mg} / \mathrm{L}$ in well 811 and $1.7 \mathrm{mg} / \mathrm{L}$ in well 808$)$, and $\mathrm{As}(0.05 \mathrm{mg} / \mathrm{L}$ in well 811$)$.

\section{COMMENT 89:}

Page 3-101, Section 3.3.2: This section needs a discussion on how the previous response actions will affect future field work and sampling strategy.

\section{RESPONSE}

Information on previous actions generally does not document whether residual levels of contamination were left behind when contaminated soils were removed. The field sampling strategy includes soil sample collection from such areas, in order to determine whether contamination remains and whether it poses a risk. 


\section{COMMENT 90:}

Page 3-101, Section 3.3.3: What technology demonstrations are currently ongoing? What types of WAG-l specific data are needed for the demonstrations, and how will the FSP accomplish this task. Also, Table 3-21, page 3-102, presents a summary of the results of the surveillance program, but is limited to external dose rate (TLD) measurements made around the Main Plant Area. In addition to external gamma dose rate measurements, a summary should be provided of the air particulate measurements, since such measurements would be a much more sensitive indicator of the presence of contaminants.

\section{RESPONSE}

DOE is committed to the development and application of innovative technology to meet compliance and cleanup goals. In DOE's 5-year plan for environmental restoration and waste management, corrective actions are Priority 1. Technology development is critical to achieving environmental goals. Activities are underway at many of the DOE installations. Specific technology demonstrations recently completed, currently underway, or planned for the near future at ORNL include the following:

- In situ stabilization of trench waste by dynamic compaction

- In situ stabilization of trench waste by grout injection

- Infiltration and biointrusion barrier over stabilized waste trenches

- In situ vitrification of soils contaminated with radionuclides

- In situ grouting of an underground storage tank

- In situ vitrification of underground storage tanks (National Demonstration at Richland, WA using ORNL waste/soils)

Additional projects underway at ORNL include the following:

- Study to assess the feasibility of manipulating the genetic potential of natural bacteria to enhance/inhibit degradation of methyl mercury in aquatic habitats

- Study to characterize the means to stimulate PCB-degrading activities of soil microorganisms, with special emphasis on anaerobic dechlorination

- Off-site study of two or three pilot-scale demonstrations of different processes for comparison of treatment efficiencies and cost for treatment of mixed-waste contaminated groundwater.

Each of the ORNL technology demonstrations has been designed so that data required to assess the feasibility of the technologies addressed are collected within the scope of the individual demonstration. Specific WAG 1 RI data needs have not yet been identified as required to support the technology demonstrations. 
As the WAG 1 RI progresses and the nature and extent of contamination are better understood, new technology demonstration projects will likely be developed by ORNL and may be integrated with WAG 1 RI data collection.

Currently, three stacks are in use near the main plant area, another two stacks are in use in Bethel Valley, and three stacks are in use in Melton Valley. Attachment A, Response to CERCLA Specific Comment 90 is a table that presents the annual airborne radioactive emissions at ORNL for all stacks except for the Oak Ridge Electron Linear Accelerator Facility (Bldg. 6010), where continuous sampling data are not currently available.

\section{COMMENT 91:}

Page 3-106, Table 3-22: Tanks were taken out of service because of leaks, but what has been done about the contamination caused by the leaks?

\section{RESPONSE}

Corrective actions outlined in Table 3-22 of the RI Plan, Revision 1, mainly refer to efforts to stop further leakage at tank sites. For the most part, remediation of soil contamination at the tank sites has not occurred. Preliminary findings of soil sampling efforts by ORNL are presented in Tables 3-11a and 3-12 in Revision 1 of the RI Plan. The RI/FS will address characterization and remediation of environmental contamination associated with inactive tanks. For additional information, refer to the Response to CERCLA Specific Comments 51 and 52.

\section{COMMENT 92:}

Page 3-109, Section 3.4: The nature and extent of environmental contamination summary should qualitatively describe the nature and extent of the environmental contamination referenced and documented in Sections 3.2 and 3.3; specifically as to how this information will impact future field activities. Need to explain in general, how Section 3.3.1.1 relates to Sections 3.3.1.2, 3.3.1.3, 3.3.1.4 and 3.3.1.5. Expand on how known WAG-l geology influences documented contamination migration, and how this impact future investigation strategy. Also, need to document potential or anticipated FSP implementation concerns.

\section{RESPONSE}

Section 3.4 is supplemented with the following text:

At least 23 leak and spill sites are within the main complex at ORNL (Grimsby, 1986). The majority of leak sites are associated with holding tanks and along pipelines serving the tanks. Spill sites are generally associated with pumping activities. Contamination of the surrounding soil ranges from the surface to as deep as $6.1 \mathrm{~m} \mathrm{(20} \mathrm{ft).} \mathrm{Evaluation} \mathrm{of} \mathrm{soil} \mathrm{borings} \mathrm{taken} \mathrm{in}$ 
the area of leak and spill sites, interviews with plant personnel, and data from the USRADS walkover survey will contribute to the execution of the Field Sampling Plan. Analyses of additional soil borings located downgradient of leak and spill sites will help define the extent of contamination.

The movement of radionuclide contaminants has been noted within pipelines and drainage systems adjacent to leak sites. This information suggests the pipelines and adjacent storm and sewer drains may serve as conduits for contaminant transport. The trenches have a greater transmissivity than the native soil and it is believed that groundwater carrying radionuclides may preferentially flow in the trenches. Fifty-one soil borings in pipeline trenches at suspected leak sites will be completed. An additional 14 borings in trenches not specifically associated with leaks are planned to test the hypothesis that contaminants have migrated within the trenches.

During the years that ORNL has been in operation, elevated concentrations of some radionuclides have been detected in White Oak Creek. The presence of these contaminants has been attributed to such factors as leaks, direct releases to White Oak Creek, movement of contaminants along trenches that discharge to the creek, and overtopping of impoundments. Evaluation of available surface water and sediment samples, interviews with plant personnel, and the USRADS walkover survey will contribute to the implementation of field sampling. The RI will include surface water and sediment analyses to confirm the nature of contaminants currently present. In addition, soil borings will be taken along transects of the White Oak Creek floodplain to evaluate historic stream contamination.

Other areas that may serve as contaminant sources include the impoundments and two former burial grounds. The impoundments generally served as holding areas for liquids contaminated with radioactive wastes. Available data from sediment samples taken in the impoundments and groundwater data from monitoring wells surrounding the impoundments will be used in implementing field sampling. Waste from one former burial ground, SWSA 2, has been removed. Soil samples taken in the area as well as monitoring wells that surround the SWSA indicate no contaminants are present. Historical data indicate that material buried at SWSA 1 are still in place. Groundwater monitoring data from the perimeter of the area, interviews with plant personnel, a review of historic air and ground photographs, and the USRADS walkover survey will be used in evaluating the likelihood of contaminant releases from SWSA 1.

The influence of the geology (Section 3.3.1.1) on documented contamination (Sections 3.3.1.2, 3.3.1.3, 3.3.1.4 and 3.3.1.5) is presented in the Response to CERCLA Specific Comment 98.

A potential FSP implementation concern is the availability of analytical laboratory services. The number of off-site, fixed-base laboratories licensed to accept and analyze samples derived from mixed hazardous and radioactive wastes is small. Should demand for their services be increased from other clients, the timelines and availability of these services may be impaired. 


\section{COMMENT 93:}

Page 3-110, Figure 3-22: This figure needs a scale.

\section{RESPONSE}

Agree.

\section{COMMENT 94:}

Page 3-111, Table 3-23: Footnotes should be re-labeled to (a), (b), (d) and (e) for consistency. Also, cross-referenced data should be documented where indicated by the table. For example, MMES (1989) is reported as the source for contaminant concentration data and is shown to be documented in Section 3.3.1.4. However, Section 3.3.1.4, Page 3-92e, states only that "ORNL has sampled the groundwater monitoring wells at WAG 1 twice. Results from the first round of sampling are available from ORNL's Environmental Monitoring and Compliance Group..." The summary of this sampling results only discusses radionuclide contamination, while there is no mention of the organic, $\mathrm{Hg}$ and other inorganic contamination indicated by Table 3-23. The above comment also applies to the following source references: Hall (1989), DEM (1986), DEM (1989), Cerling and Spalding (1981), Cerling and Huff (1986), Morrison and Cerling (1987), and Cutshall (1985). Finally, the referenced source Ketelle (1986) should be Ketelle, et al. (1986); and the referenced source Huang, et al. (1984) should be Huang, et al. (1984a).

\section{RESPONSE}

A revised table is shown in Attachment A, Response to CERCLA Specific Comment 94. Footnotes have been relabeled (a), (b), (c), (d), and (e). DEM (1986) and (1989) have been replaced by MMES (1987). Hall (1989) has been dropped from the table. Huang et al. (1984) has been changed to Huang et al. (1984a) and Ketelle (1986) to Ketelle et al. (1986).

Section 3.4 is supplemented with the following text:

\section{Section 3.3.1.4 Groundwater}

ORNL performed two rounds of sampling at WAG 1 perimeter groundwater monitoring wells prior to the submission of the WAG 1 RI Plan (Revision 1). Analyses were run for groundwater quality constituents listed in $40 \mathrm{CFR} 265$, gross alpha, gross beta, ${ }^{60} \mathrm{Co},{ }^{137} \mathrm{Cs}$, total radioactive $\mathrm{Sr}$, total $\mathrm{Ra}$, and ${ }^{3} \mathrm{H}$. Maximum concentrations of these contaminants are shown in Attachment A, Response to CERCLA Specific Comment 94 . Well 812 had the highest gross alpha $(8.6 \mathrm{~Bq} / \mathrm{L})$, gross beta $(660 \mathrm{~Bq} / \mathrm{L})$, and radioactive $\mathrm{Sr}(280 \mathrm{~Bq} / \mathrm{L})$. Parameters exceeding the National Primary Drinking Water Limits include fecal coliform (3 COL/100 mL in Wells 822 and 827), fluoride $(3.8 \mathrm{mg} / \mathrm{L}$ in Well 811 and $1.7 \mathrm{mg} / \mathrm{L}$ in Well $808)$, and As $(0.05 \mathrm{mg} / \mathrm{L}$ in Well 811$)$. Mercury was not present in amounts exceeding the 
National Primary Drinking Water limits. The maximum concentration of organic halides (total) was $0.16 \mathrm{mg} / \mathrm{L}$.

\section{Section 3.3.1.3 Sediments}

Stream gravel surveys in the WOC watershed were conducted by Cerling and Spalding (1981) to define the areal distribution of ${ }^{60} \mathrm{Co},{ }^{137} \mathrm{Cs}$, and ${ }^{90} \mathrm{Sr}$. Later studies (Cerling and Huff, 1986; Morrison and Cerling, 1987) corroborated the general findings of the earlier study. Attachment A, Response to CERCLA Specific Comment 94 gives the ranges of concentrations of various radionuclides found in White Oak Creek, First Creek, and Northwest Tributary sediments (Fifth Creek sediments were not sampled). The principal source of ${ }^{137} \mathrm{Cs}$ is the Process Waste Treatment Plant. Sampling locations 383-385 along First Creek are downstream of locations $400-412$ and show elevated ${ }^{137} \mathrm{Cs}$ activity. Cesium-137 contamination in stream gravels at these locations may originate from a ${ }^{137} \mathrm{Cs}$ seep along the lower reaches of First Creek. Mercury was not analyzed in these studies.

Various fission products were identified in Fifth Creek, including ${ }^{115} \mathrm{Cd},{ }^{137} \mathrm{Cs},{ }^{152} \mathrm{Eu},{ }^{154} \mathrm{Eu}$, ${ }^{153} \mathrm{Gd}$, and ${ }^{60} \mathrm{Co}$ (Cutshall, 1985) (See Attachment A, Response to CERCLA Specific Comment 94 for location map). While activity levels of the various radioisotopes are not clear from the report, Cutshall (1985) states that the contaminant level is several orders of magnitude lower than that typically measured in lower White Oak Creek. According to the report, ${ }^{115} \mathrm{Cd}$ appears to enter the creek between stations 5 and 6 , the reach adjacent to the Oak Ridge Reactor cooling towers. ${ }^{137} \mathrm{Cs}$ is added to the creek between stations 5 and 6 and again between stations 3 and $4 .{ }^{152} \mathrm{Eu}$ enters the creek between stations 3 and $4 .{ }^{60} \mathrm{Co}$ enters the creek between stations 5 and 6 , perhaps between stations 3 and 4 and probably between stations 2 and 3. The unique contamination patterns in Fifth Creek indicate that reactor coolant contributes ${ }^{60} \mathrm{Co},{ }^{115} \mathrm{Cd},{ }^{137} \mathrm{Cs}$, and ${ }^{152} \mathrm{Eu}$ to Fifth Creek. Building 3047 may contribute ${ }^{152} \mathrm{Eu},{ }^{137} \mathrm{Cs}$, and ${ }^{60} \mathrm{Co}$. Another source of ${ }^{60} \mathrm{Co}$ may be from the east side of Building 3500. Mercury was not analyzed during this study.

\section{COMMENT 95:}

Page 3-109, Para. 1: Need to summarize Grimsby (1986) report in greater detail, including concentrations of contaminants.

\section{RESPONSE}

This report is summarized in the Response to CERCLA Specific Comment 92.

\section{COMMENT 96:}

Page 3-109, Para. 1, Line 11: Specify the type and concentration of contaminated discharge to WAG-l streams. 


\section{RESPONSE}

The RI Plan is supplemented with the following text:

The type, concentration, and source of contaminated discharge to WAG 1 streams are poorly documented. Some of the more reliably documented discharges to ORNL streams include an April 1959 discharge of $70 \mathrm{Ci}$ of ${ }^{147} \mathrm{Pm}$ from the General Isotopes Area, April 1962 discharge of $300 \mathrm{mCi}$ of ${ }^{90} \mathrm{Sr}$ from a leak in the General Isotopes Area, and a February 1961 discharge of $2.5 \mathrm{Ci}$ (unspecified radionuclide) from a leak(s) in the 3024 area. In $1950,65 \mathrm{Ci}$ of the total $169 \mathrm{Ci}$ total released to WOC was attributed to leak(s) from the 3026 area.

While discharges from pipeline trenches have been noted in all streams at ORNL, these discharges have not been sampled to determine the presence or type of contaminants. These activities will be conducted as part of the surface water sampling activities.

\section{COMMENT 97:}

Page 3-109. Para. 2: Also need to include metals analysis in scoping efforts.

\section{RESPONSE}

Metals analyses are included in the FSP. See specifically Attachment A-4 in Revision 1 of the RI Plan.

\section{COMMENT 98:}

Page 112, Section 3.5: The conceptual model of WAG-1 discussion should be restructured to present all known facts undocumented by the RI Plan and the information provided in Sections 3.1, 3.2, 3.3 and 3.4. Specifically, comments should address in general, how present understanding of WAG-l geology and contamination will dictate future operable unit specific investigation strategy and goals. Where data gaps exist, this section should describe how the RI Plan and FSP will address those gaps. In addition, the conceptual WAG-1 model should be fully supported by Section 3.4.

\section{RESPONSE}

The revised conceptual model is presented in the following paragraphs as a supplement to the RI Plan:

ORNL is a large research and development facility comprised of research laboratories, isotope production facilities, associated administrative and support facilities, a number of radiological process impoundments, a coal-fired heating plant, and all associated process, heating and ventilation, water, electrical, and waste lines. At a minimum, the upper $3.1 \mathrm{~m}(10.2 \mathrm{ft})$ of 
soil underlying the facility has been thoroughly disrupted by construction activities over the last 45 years. Pipe trenches cross the site from one end to the other in all directions but most commonly parallel streets. The pipelines themselves are located at various depths, generally dependent upon function.

The facility, located in Bethel Valley (See Attachment A, Response to CERCLA Specific Comment 64), is underlain by rocks, primarily limestone, of the Chickamauga Group, which strike approximately $\mathrm{N}^{\circ} 5^{\circ} \mathrm{E}$ with an average dip of $40^{\circ} \mathrm{SE}$. Haw Ridge southeast of WAG 1 is underlain by the Copper Creek thrust fault, which places the Rome Formation in fault contact with the Chickamauga Group. Chestnut Ridge to the northwest is underlain by rocks of the Knox Group. The surface of WAG 1 is drained by WOC and its tributaries, First and Fifth Creeks. To the northwest of Central Avenue, most buried pipelines and building basements are above the water table; southeast of Central Avenue, they are below the water table.

In development of a preliminary conceptual model for WAG 1, the interaction between known and potential contaminant sources and environmental media has been considered. This interaction is depicted schematically in Attachment A, Response to CERCLA Specific Comment 98. Primary source areas consist of potentially-leaking tanks, lines, and burial grounds, with secondary sources including soils and sediments contaminated from previous ORNL activities. Where the water table is below the overburden-bedrock interface, there will likely be only limited geologic influence on contamination migration; most of the controls are man-made. Where pipe trenches and building basements are below the water table, contaminant flow will be controlled by the more permeable material in pipe trenches, but dispersion and diffusion will lead to a broader contaminant distribution. Pipe trenches may cause contaminant migration in directions other those expected by water table gradients alone.

WOC originates to the northeast of WAG 1 on Chestnut Ridge. Base flow in WOC may decrease in the dry season on the upper reaches of the ridge but almost certainly increases throughout the year in the vicinity of WAG 1 due to continuous discharge from operations at ORNL. Shallow (overburden) groundwater flow is believed to generally mimic topography toward WOC and First and Fifth Creeks. Divides may occur in the northwest portion of the site. Flow in the bedrock is believed to be generally tortuous northeast - southwest along the strike and occurs along bedding planes, joints and fractures, and solution channels at a depth of 1.5 to $6.1 \mathrm{~m}$ ( 4.9 to $20.0 \mathrm{ft}$ ). The permeability of the rock decreases with depth, and there is some evidence of an upward gradient in the deeper portions of the rock. Across the entire plant site the shallow groundwater, the surface water system, sediments, and sections of soil have been degraded by site activities.

The proposed Phase I site investigation has been designed primarily to identify contaminants, confirm source areas, and define contaminant pathway hypotheses. A preliminary concept of operable units has been developed to facilitate evaluation of potential remedial action options. In developing the preliminary operable unit design, ORNL has chosen to examine potential primary contaminant sources within environmental media. Where potential sources can be hydrogeologically isolated to facilitate investigation, that has been done. For example, 
SWSA 1 is located on the southern boundary of WAG 1. Any required remediation of this unit probably can be addressed directly, without concern for its interaction with plant operations or other identified SWMUs. By contrast, in remediation of a release from an inactive tank in one of the underground tank farms, media are likely to be contaminated by many sources, both primary and secondary. Personnel health and safety concerns and ongoing plant operations may require that final disposition of some units await overall plant closure activities. As more is learned about individual sources and their interaction with transport mechanisms, the definition of tentative operable units will be refined. For example, an abandoned pipeline might be pressure grouted if is found to act as a conduit for contaminated groundwater.

Groundwater at WAG 1 is currently identified as a preliminary operable unit with three subdivisions. Any necessary remediation of the aquifer at WAG 1 may require different approaches according to depth. For example, the pump and treat method is relatively effective in remediating permeable unconsolidated soil aquifers. However, only a portion of the facility aquifer fits this description. In addition, while water table portions of the upper bedrock aquifer have been degraded, deeper portions of the aquifer [depths greater than $30.5 \mathrm{~m}$ $(100.0 \mathrm{ft})]$ may not be contaminated.

Working hypotheses and data gaps identified by the literature review have been developed. The objectives of the FSP are to sample the environmental media, to establish how the various media interact in a setting substantially modified by human influences, and to aid in the development of exposure point locations. This field sampling and data analysis will resolve the working hypotheses and data gaps.

\section{COMMENT 99:}

Page 3-112, Para. 2: Need to refer to a figure depicting Bethel Valley.

\section{RESPONSE}

Refer to Attachment A, Response to CERCLA Specific Comment 64.

\section{COMMENT 100:}

Page 3-113, Para. 2, Line 1: What types of investigations will be initiated to fully define points of potential exposure.

\section{RESPONSE}

Points of potential exposure for assessing risks attributable to the SWMUs referred to in the comment will be identified in two ways. First, a generic exposure pathway scenario is currently being developed for use in assessing each SWMU or cluster of closely associated 
SWMUs. This scenario will address the risks associated with ingestion of groundwater from the immediate downgradient vicinity of the SWMU. It will also address risks from soil ingestion and direct radiation exposure. Second, any site-specific exposure routes, identified through site investigation, such as basement sumps or direct discharges to environmental media both on-site and off-site, such as groundwater seeps entering streams, will be included as potential exposure pathways.

\section{COMMENT 101:}

Page 4-2, Para. 1, Bullet 2: ATSDR should be consulted to determine the information necessary for the ORR Health Assessment that $\underline{A T S D R}$ must complete.

\section{RESPONSE}

Information is being gathered by the ORNL Office of Risk Analysis pursuant to OSWER Directive 9285.1-02. This office will provide the data to ATSDR.

\section{COMMENT 102:}

Page 4-3, Table 4-1: This table presents the various Operable Units in which WAG-l has been divided in order to facilitate the performance of the RI. The groupings are according to major environmental components (i.e., groundwater, sediments, soil, tanks, impoundments). This method of defining Operable Units is acceptable at this stage in the RI/FS process but is not consistent with the definition and purpose of an operable unit as described on page 4-1 and the discussion on page 4-4. Ultimately, Operable Units should be defined according to contamination that is established based on sampling and analysis and that requires corrective/remedial action. The bulleted items under the Groundwater Operable Unit should be modified to read as follows:

- Shallow (stormflow)

- Intermediate (water table)

- Deep (karst and fractured bedrock).

\section{RESPONSE}

The table of preliminary operable units has been revised.

The initial identification of proposed operable units in WAG 1 was based on the need for a methodical evaluation of the site problems. The groupings also provide manageable units for an initial approach for the preliminary risk assessment. Each preliminary operable unit is discrete and identifiabie, to the extent thus far possibie for such a compiex site. 
Waste media and physical or hydrological relationships were used to group the WAG 1 SWMUs.

The Main Plant Area has been designated as a tentative operable unit because the variety of contaminant sources within the area appear to be hydrologically interrelated. Existing data indicate that transport of materials from discrete sources has occurred throughout the main plant via man-made structures. This dispersion of contaminants has resulted in the possibility that the entire area may require remediation. The subdivisions within the main plant site were undertaken to direct assessment efforts upon areas where the inventory of contaminants and the "waste media" are thought to be similar.

"Waste media" include obvious physical features such as tanks, waste storage and accumulation areas, and SWSAs, as well as soils, sediments, and liquid media. The radiological ponds and impoundments seemed clearly appropriate as an operable unit. However, groundwater may be hydraulically connected with impoundment liquids and contaminated soils.

The SWMU groupings also considered similarities (e.g., geographic location, hydrologic connections, process and/or functional equivalencies, and nature of potential contamination) allowing them to be treated similarly or in combination with other SWMUs if remediation is necessary. Media that have different physical characteristics but are presently mixed (or so closely associated that any change in one affects the other) are logically addressed in one operable unit.

The designation and/or composition of the operable units will be refined as data from the Phase I RI for WAG 1 are evaluated. Some SWMUs may prove to be hydrologically connected to units in a way that is not obvious at present.

COMMENT 103:

Page 4-5, Section 4.2: The title of this section should be changed to the following: "Preliminary Risk Assessment Process". Also, throughout this section replace the phrase "health assessment" with "risk assessment".

\section{RESPONSE}

Agree.

\section{COMMENT 104:}

Puge 4-5, Section 4.2: The environmental mobility of radioactive isotopes should be evaluated and considered in the RI Plan. Tritium and possibly carbon-14 and other anionic radioactive isotopes are generally more mobile than the transuranics (uranium), or other 
rare earth species. How environmental mobility impacts the study and the proposed sampling program needs to be addressed in this RI Plan and integrated with the FS phase of the program.

\section{RESPONSE}

DOE agrees that mobile radionuclides are significant parameters to this investigation.

Carbon-14 is not believed to be a contaminant of concern in WAG 1. Tritium, perhaps the most conservative of all such radionuclides, is routinely analyzed in all liquid samples. This material serves as an important indication of the potential presence of other radionuclides. Another fairly mobile radionuclide is strontium. Other radionuclides, such as the transuranics and cesium, tend to be sorbed into soil particles.

In evaluating soils, the presence of the higher valence transuranics and absence of the mobile constituents are an indication of the degree to which radionuclides may be sorbed. This provides an initial assessment of the intermedia transfer potential of contaminated soils and sediments.

More detailed evaluations of the intermedia transfer potential of soils and sediments will be conducted during Phase II studies as required to support the FS. The TCLP method (using a leaching media (water) that is characteristic of in situ conditions) will be used to further quantify leaching potential. This will aid the FS by identifying contaminated soils that may warrant either removal or in situ treatment or isolation.

COMMENT 105:

Page 4-5, Para. 3, Line 3: Need to add the term "risk" after the term "baseline".

\section{RESPONSE}

Agree.

COMMENT 106:

Page 4-5, Para. 3, Line 4: Need to add the phrase "and the environment" following "human health".

\section{RESPONSE}

Agree. 
COMMENT 107:

Page 4-5, Para. 3, Line 6: Need to replace the phrase "corrective measures" with

"response actions".

RESPONSE

Agree.

COMMENT 108:

Page 4-5, Para. 3, Line 7: Need to replace the phrase "performing corrective measures" with "No Further Action decisions".

RESPONSE

Agree.

COMMENT 109:

Page 4-6, Line 1: Add the term "two" before the term "phases".

\section{RESPONSE}

Agree.

COMMENT 110:

Page 4-6, Para. 1, Line 1: Replace the term "health" with "risk".

\section{RESPONSE}

Agree.

COMMENT 111:

Page 4-6, Para. 1: Replace the sentence "The guidance that will-be followed in developing the baseline health assessment is the RI/FS Guidance (EPA, 1988b), the Superfund Public Health Evaluation Manual (EPA, 1986), and the CERCLA Compliance With Other Laws

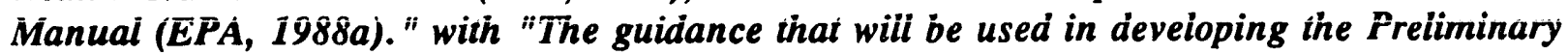
Risk Analysis includes the Conducting Remedial Investigations/Feasibility Studies Under 
CERCLA Guidance (U.S. EPA, 1988b), Parts I and II of the CERCLA Compliance With Other Laws Manual (U.S. EPA, 1988a and 1989a), Volumes I and II of the Risk Assessment Guidance for Superfund (U.S. EPA, 1989b and 1989c) and the Superfund Exposure Assessment Manual (U.S. EPA, 1988c). Need to correct and update the references on Pages $R-8$ and $R-9$. Also, while it is true that Baseline Risk Assessments are used to establish health based remediation criteria in the absence of ARARs, they are also used to help identify the most appropriate remedial action strategy by comparing the risks associated with each alternative considered in the FS detailed analysis. Accordingly, Baseline Risk Assessments are not only required in the absence of ARARs, but are instead always required for specific Operable Units during RI/FS activity.

\section{RESPONSE}

The guidance that will be used in developing the preliminary risk assessment is Volumes I and II of the Risk Assessment Guidance for Superfund (EPA, 1989), the Superfund Exposure Assessment Manual (EPA, 1988a), and the Guidance for Conducting Remedial Investigations/Feasibility Studies Under CERCLA (EPA, 1988b).

\section{COMMENT 112:}

Page 4-6, Para. 2, Line 4: Need to add the phrase ", or environmental impact" after "human intake or exposure".

\section{RESPONSE}

Agree.

COMMENT 113:

Page 4-6, Para. 3, Line 8: Need to add "and the environment" after the phrase "human health".

\section{RESPONSE}

Agree.

COMMENT 114:

Pages 4-7 to 4-9, Figure 4-1: This figure should include potential biological receptors other than humans. 


\section{RESPONSE}

Agree.

COMMENT 115:

Page 4-10, Para. 2, Line 1: Need to add the term "and" between the terms "human" and "environmental".

RESPONSE

Agree. This was a typographical error.

COMMENT 116:

Page 4-10, Para. 2, Line 3: Need to add the term "and" between the terms "human" and "environmental".

\section{RESPONSE}

Agree. This was a typographical error.

COMMENT 117:

Page 4-10, Para. 2, Line 5: Need to replace the phrase "public health evaluation" with "risk assessment".

RESPONSE

Agree.

COMMENT 118:

Page 4-10, Para. 2, Line 6: Need to delete the sentence "Other exposure routes, which have not been identified, may be insignificant when compared to the effect from these identified exposure routes.".

\section{RESPONSE}

Agree. 
COMMENT 119:

Page 4-10, Para. 2, Line 10: Need to replace the phrase "baseline public health evaluation" with "preliminary risk analysis".

\section{RESPONSE}

Agree.

\section{COMMENT 120:}

Page 4-11, Table 4-2: For potential "Boundary Receptors" it is appropriate to include the "ingestion" route of exposure under the groundwater and surface water pathways, and the direct radiation route under the sediment pathway. All three pathways should be assessed for chemical and radiological components.

\section{RESPONSE}

Agree.

COMMENT 121:

Page 4-11, Table 4-2: For potential "ORNL Employees" it is appropriate to include the "direct radiation" route of exposure under the sediments pathway. The sediments pathway should be assessed for chemical and radiological components.

\section{RESPONSE}

Agree.

\section{COMMENT 122:}

Page 4-11, Table 4-2, Potential Receptors Column: This column should also include a evaluation of the direct risk potential relative to sensitive environments (e.g., wetlands), and potentially affected flora and fauna.

\section{RESPONSE}

Agree. 
COMMENT 123:

Page 4-12, Bulleted Items: Need to add a discussion of a exposure scenario that considers the potential risk to sensitive environments and biota.

\section{RESPONSE}

The following text is a supplement to the RI Plan:

An exposure scenario consists of a source, pathway, and receptor. Potential environmental exposure routes for WAG 1 environmental receptors are shown in Attachment A, Response to CERCLA Specific Comment 123. Existing physical and chemical data, along with data collected during the RI for various media, will be used to characterize contaminant source areas and release mechanisms at WAG 1 . During the environmental evaluation, activities will include:

- examination of contaminant release sources and release media;

- examination of the environmental transport media;

- identification of exposure points; and

- identification of exposure routes for biota.

\section{COMMENT 124:}

Page 4-13, Table 4-3, Potential Receptor Column: Need to add a receptor identification which represents the exposure scenario concerning the potential risk to sensitive environments and biota.

\section{RESPONSE}

See Response to CERCLA Specific Comment 123.

COMMENT 125:

Page 4-14, Para. 1, Line 4: Need to add the phrase "or the environment" after "human health".

\section{RESPONSE}

Agree. 


\section{COMMENT 126:}

Page 4-15, Table 4-4 and Page 5-3, Figure 5-1: Table 4-4 and Figure 5-1 should also consider other geophysical methods (e.g., resistivity, seismic refraction and Ground Penetrating Radar) to characterize bedrock with fracture zones and karst features, and to correlate with the models derived from other screening investigation interpretations (e.g., EM-31 and corehole geophysics).

\section{RESPONSE}

Man-made influences and other factors at WAG 1 negate the use of geophysical surveying methods such as seismic refraction, resistivity, and ground penetrating radar. See Responses to CERCLA Specific Comments 7 and 21 for more detailed discussions.

\section{COMMENT 127:}

Pages 4-15 to 4-16b, Table 4-4 and Page 5-3, Figure 5-1: The WAG-l RI Plan should provide timelines that associate the FSP tasks identified in Table 4-4 and Figure 5-1, including some addition tasks not indicated by the table and figure, into two phases of investigation (i.e., RI Phase I and RI Phase II). Table 4-4 and Figure 5-1 should indicate the following $R I$ phases and their anticipated time duration:

\section{WAG-1-specific RI Phase I}

* review and incorporation of existing information,

- radiation walkover survey,

- X-ray fluorescence,

* surface geophysical surveys,

- headspace gas analysis,

- evaluation and upgrade of existing wells, and drywell and sump sampling,

- existing groundwater well network sampling, including water level and flowrate monitoring,

- creek channel sediment sampling,

- creek sampling,

- creek flow monitoring,

- impoundment sediment sampling,

- deep coreholes transect,

- Soils I sampling,

- Soils II sampling,

- personnel exposure measurements,

- receptor location determinations, 
and, for those WAG 1 contaminant sources/releases that do not justify a No Further Action decision,

\section{Operable Unit-specific RI Phase II}

* Soils III sampling and the expansion of the existing groundwater well network, and additional media sampling, as appropriate,

* treatability studies,

* bioassay,

The above tasks identified with asterisks should be included in Table 4-4 and Figure 5-1, and described in the text of the RI Plan.

\section{RESPONSE}

See Responses to CERCLA Specific Comments 26 and 27.

\section{COMMENT 128:}

Page 4-16c, Para. 1: This paragraph should be deleted. The preliminary risk analysis must address concurrently, the potential threat to human health and the environment on a operable unit-specific basis. Also, the above bulleted items, and the comments of Section 4.2.2 on page 4-14 should be incorporated into Section 4.2.1. The RI Plan should provide a discussion of the data needs for the bioassay task.

\section{RESPONSE}

DOE agrees with the deletion of paragraph 1. However, DOE disagrees that the preliminary risk assessment should be performed on an operable unit-specific basis as part of Phase I activities. The assessment should be operable unit-specific as part of Phase II activities.

DOE agrees that the bulleted items and comments on Section 4.2.2 should be incorporated into Section 4.2.1. Data needs for bioassay task are as described in Response to CERCLA General Comment 10.

COMMENT 129:

Page 4-20, Table 4-6: For in-place containment, capping, leachability should be checked under soils characterization.

\section{RESPONSE}

These will be considered in the evaluation. 
COMMENT 130:

Page 4-20, Table 4-6: For in-place containment, vertical barriers, porosity should be checked under soils characterization.

RESPONSE

Agree.

COMMENT 131:

Page 4-20, Table 4-6: The RI Plan should also consider the data needs listed under biota characterization to support the bioassay.

\section{RESPONSE}

Information on the impact of ORNL operations and environmental releases on biota are being collected under the ongoing Biota Monitoring and Abatement Program at ORNL. The RI project will evaluate the extent to which these data support the objectives of the RI. These data will be used, supplemented by new data collected by the RI program if necessary, to complete the environmental assessment component of the Preliminary Risk Assessment Report.

\section{COMMENT 132:}

Page 4-21, Section 4.3.2: This section should include a discussion of how the FSP will satisfy the data needs summarized in Table 4-6. The discussion should include a statement of the anticipated problems or any special considerations in obtaining the RI data.

\section{RESPONSE}

Data obtained through individual field sampling campaigns will be used to evaluate potential remedial alternatives. For example, groundwater levels measured monthly in over 160 locations when combined with geologic characterization data will be used to construct groundwater flow nets to estimate flow directions and specific discharges. This groundwater characterization information will be used in conjunction with data on facility configurations and operations (e.g., pipeline construction and orientations) and release characterization to assess the potential effectiveness of a variety of site remediation alternatives.

DOE does not anticipate any problems at this time in obtaining the characterization data needed to perform an initial assessment of remedial alternatives. The program is deliberately structured as a phased strategy of data collection, synthesis, and evaluation. At the conclusion of Phase I, any specific data needed to more fully evaluate remedial alternatives during 
operable unit-specific studies under Phase II will be identified. Treatability studies will be identified to be conducted during Phase II for unique waste treatment scenarios. At the conclusion of Phase I it will be possible to integrate literature evaluation of innovative treatment technologies into future data collection activities.

COMMENT 133:

Page 5-1, Para. 3, Line 2: Replace the phrase "heavy metal contamination" with "hazardous constituent contamination".

\section{RESPONSE}

Agree.

COMMENT 134:

Page 5-1, Para. 3, Line 6: Replace the phrase "contaminants, as well as for volatile organic compounds" with "hazardous substances..

\section{RESPONSE}

Agree.

COMMENT 135:

Page 5-2, Line 1: Replace the term "materials" with "hazardous substances".

\section{RESPONSE}

Agree.

COMMENT 136:

Page 5-2, Para. 1, Line 7: Replace the phrase "discharge to the surface" with "through environmental media."

\section{RESPONSE}

Agree. 
COMMENT 137:

Page 5-2, Para. 2, Line 11: Integrity demonstrations are necessary for tanks and pipelines. These demonstrations will correlate with screening activity results. This RI Plan needs to provide the details for integrity demonstrations (e.g., tracer tests) as part of RI Phase I activities.

\section{RESPONSE}

Tank integrity demonstrations will be conducted pursuant to Section IX of the proposed FFA under a separate program at ORNL. They are not performed under the WAG 1 RI.

However, data from these demonstrations will be used in identifying operable units and in completing the RI Reports (Preliminary Risk Assessment and Preliminary Site Characterization Summary Report).

DOE does not anticipate use of additional tracer test studies during Phase I. Given the uncertainty in the nature and extent of releases and the site-specific groundwater flow patterns, any pressure tests used to assess pipeline integrity during Phase I offer a risk of spreading contamination. Such tests, if performed, will be careîlly designed and monitored.

\section{COMMENT 138:}

Page 5-2, Para. 2, Line 16: The evaluation of the proximity of pipeline trenches to creeks needs to be completed and discussed in this RI Plan. The results should be incorporated into RI Phase I activities.

\section{RESPONSE}

As part of the WAG 1 Phase I RI, the location of pipeline trenches in conjunction with their proximity to creeks will be evaluated to assess the trenches as preferential pathways for contaminant discharge from groundwater to surface water. It is anticipated that trench backfill will be sampled for the presence of radionuclides, organics, and other contaminants of concern.

\section{COMMENT 139:}

Page 5-3, Figure 5-1: See Comments \#126 and \#127 above.

\section{RESPONSE}

Please see Responses to CERCLA Specific Comments 126 and 127. 
COMMENT 140:

Page 5-3, Figure 5-1: Need to add "Bioassay" to the Model/Pathway Evaluation activities. RESPONSE

Agree.

\section{COMMENT 141:}

Page 5-5, Para. 1, Line 1: The planned "additional groundwater activities" to determine aquifer properties should be specified by the RI Plan.

\section{RESPONSE}

Data from the existing coreholes and seven new coreholes, including geophysical logs, rock core, and packer tests, will be obtained and evaluated to confirm the presence of an upward vertical gradient in the deep subsurface.

\section{COMMENT 142:}

Page 5-5, Para. 3, Line 5: Section A7.0 of the FSP should provide a figure showing the locations of all background samples.

\section{RESPONSE}

See Attachment A, Response to CERCLA Specific Comment 143 for maps illustrating reference (background) sampling locations.

\section{COMMENT 143:}

Page 5-5, Para. 3, Line 11: Need to discuss the sampling strategy to determine the significance concerning the variation in background concentrations from all media. Section A7.0 of the FSP should provide the details of the strategy.

\section{RESPONSE}

The sampling strategy to determine the significance concerning the variation in background (ñow referied to as reference) concentrations from all media has been revised and is supplied as a supplement to the Field Sampling Plan in Attachment A, Response to CERCLA Specific Comment 143. 


\section{COMMENT 144:}

Page 5-6, Section 5.1.2: Treatability studies should be conducted as part of RI Phase II activities. The Preliminary Characterization Summary Reports should provide the Development of Potential Remedial Alternatives and recommendations for treatability studies necessary to support the Feasibility Studies.

\section{RESPONSE}

Noted. Please refer to the Response to CERCLA Specific General Comment 14.

\section{COMMENT 145:}

Page 5-6, Section 5.1.3.1, Line 3: Replace the phrase "could be used" with "will be used". This section needs more detail, or needs to include a reference to a supporting document (i.e., "Environmental, Safety and Health Plan"). ES\&H monitoring during RI field activities should include radiological surveys for alpha, beta and gamma radiation.

\section{RESPONSE}

The following supplement is added to the text in the RI Plan:

The RI/FS project ES\&H Plan has been finalized and establishes the overall organization, interfaces, criteria, and guidance for ensuring that RI/FS project activities comply with federal, state, and DOE laws, regulations, orders, requirements, and procedures. A WAG 1specific ES\&H Plan meeting the requirements specified on OSHA 29 CFR 1910.120 has been prepared for discrete elements of work at the site. ES\&H personnel will routinely, monitor for alpha, beta, and gamma radiation during field activities.

\section{COMMENT 146:}

Page 5-7, Section 5.1.3.2: The inactive tank sampling protocols should be presented as part of the RI Plan or a supporting document referenced by the RI Plan.

\section{RESPONSE}

Inactive tank content characterization is a specific task pursuant to Section IX of the proposed FFA, performed by other personnel. However, information obtained from this sampling activity is an important component of the information used in identifying operable units, and in completing the RI Reports. These protocols are presented in "Sampling and Analysis of the Inactive Waste Storage Tank Contents at ORNL", ORNL/RAP-53 by J. W. Autrey, et al. A 
copy will be provided under separate cover along with the reports cited in Responses to CERCLA Specific Comment 80 and Part A-RCRA Specific Comment 17.

\section{COMMENT 147:}

Page 5-7, Section 5.1.4: What is meant by the phrase "allowable concentrations"? The RI Plan needs to give more detail concerning the methods to be used to develop allowable concentrations. Also, the Preliminary Characterization Summary Reports should propose Operable Unit-specific ARARs, which will be further refined and finalized during the $R I$ Phase II.

\section{RESPONSE}

This phrase refers to hazardous substance concentrations identified through the ARAR process, and through the development of remedial action goals.

Section 121 of CERCLA requires that, subject to specified exceptions, remedial action must be in compliance with ARARs and TBCs. ARARs and TBCs are identified at several steps in the remedy selection process. Section XXI (F) of the proposed FFA for the environmental restoration of the ORR calls for the preparation of a draft listing of all ARARs as mandated by $\$ 121$ of SARA. Pursuant to this requirement, chemical and location-specific ARARs and TBC guidance will be submitted to EPA and TDC for review during the RI. As additional site information is developed, other ARARs and TBCs may be identified, and the list of "potential" ARARs and TBCs will be further refined.

\section{COMMENT 148:}

Page 5-8, Section 5.2.1, Bulleted Items: This section should include a discussion of why the models of choice for WAG-l are particularly suited for WAG-l. Need to discuss any anticipated problems and necessary $R I$ investigations to support the modeling or risk analysis. Include a summary of the FSP investigations necessary to accomplish specific RI Plan modeling and risk analysis objectives. Also, What is the basis for selection of the Gilbert Model to derive allowable concentrations of radionuclides?

Page 5-9, Section 5.2.2: This section needs a discussion, or should reference an appropriate document that describes the techniques that will be used for statistical analysis.

\section{RESPONSE}

The contaminant transport models identified for possible use in performing the contaminant transport in WAG 1 include:

- $\quad$ surface water flow and transport: CREAMS, HSPF, SWMM 
- groundwater flow and transport: SWIFT II, FEMWATER, BLT

- geochemistry: PHREEQE

Considering the conceptual model for WAG 1, these models exhibit appropriate capabilities. The surface waters in WAG 1 include small streams which are highly responsive to rainfall events; they carry runoff from impervious areas and have large interflow and baseflow (groundwater discharge) components. The unsaturated zone on the site is highly disturbed due to the existence of a complex network of underground conduits and pipes. The CREAMS model would be useful as a screening level model which would provide a water balance within the root zone and provide estimates of runoff and percolation. However, only models such as EPA's HSPF or SWMM, provide the capability to calculate interflow and baseflow components of runoff.

CREAMS is a field scale model, yet HSPF and SWMM both are distributed models which can simulate flow and contaminant transport in surface waters including conduits. Both models can handle point and non-point source contaminants (conservative and non-conservative constituents).

The active groundwater on-site occurs both in saprolite and in the bedrock. While saprolite is a heterogeneous porous media, where groundwater flow and direction are determined by hydraulic gradient and media porosity, the bedrock is a fractured media where flow occurs in a secondary porosity features such as fractures. FEMWATER and BLT are two-dimensional models (flow and transport) suitable for modeling in unsaturated and saturated porous media. BLT also includes a source term component to compute leachate rates from containers. SWIFT II is a three-dimensional model capable of modeling density-dependent groundwater flow, and transport of radionuclides in anisotropic, heterogeneous porous media and fractured rock. Various hydrogeochemical models are available for calculating the composition of solutions in equilibrium with multiple phases. If need arises for a geochemical reaction model, the PHREEQE model by the U.S. Geological Survey will be used.

Field Sampling Plan investigations in support of modeling include hydrogeologic field tests to determine various model parameters, surface water and groundwater; and soil and sediment.

The Gilbert Model will no longer be used to derive allowable concentrations for radionuclides. Instead, a model has been developed which uses EPA-approved slope factors from the Health Effects Assessment Summary Tables. This new model was developed in accordance with the Risk Assessment Guidance used by the CERCLA program at EPA. Dose is also calculated in accordance with this guidance manual.

See Response to CERCLA Specific Comment 149 for a discussion of statistical methods to be employed. 


\section{COMMENT 149:}

Page 5-9, Section 5.2.2: This section needs a discussion, or should reference an appropriate document that describes the techniques that will be used for statistical analysis.

\section{RESPONSE}

Raw data will be analyzed with available statistical techniques to derive useful correlations, trends, averages, etc. Standard statistical methods will provide statistical, graphical, and reporting capabilities. The SAS system will be used to calculate the arithmetic mean and the population standard deviation. Methods and references in RAGS Risk Assessment Guidance for Superfund, Volume 1, (EPA, 1989) Human Health Evaluation Manual (Part A) will be used to estimate the upper confidence limit (i.e., the 95 percent upper confidence limit on the arithmetic mean).

\section{COMMENT 150:}

Page 5-9, Section 5.3: This section should include a discussion of the generic studies that are appropriate for WAG-1-specific RI objectives. Also, the RI Plan should incorporate the generic studies as part of either RI Phase I or Phase II investigations and include a discussion of the nature an extent of the investigations.

\section{RESPONSE}

Generic studies may be performed as a cost-effective approach for addressing techniques or issues that apply to more than one WAG or that are needed to complete the FS. Such studies may include an ecological investigation, a sediment transport study for White Oak Creek basin, waste and water treatment studies, containment design studies, migration analysis studies, off-site disposal studies, and regulatory strategy studies. Generic studies will be identified as part of the Phase I work and will be incorporated into Phase II activities.

\section{COMMENT 151:}

Page 5-9, Section 5.4: This section should provide examples, or should reference an appropriate document that provides the forms to be used for data summary presentations.

\section{RESPONSE}

A wide variety of data will be collected as a result of site characterization, remote sensing, and intrusive sampling. The project will also generate substantial data supporting risk assessments, on-site health and safety monitoring, alternatives analyses, and QA/QC implementation. 
Combinations of tabular and graphic displays will be used to display such extensive and varied data. Specific data presentation formats will be developed by using the methods recommended in the RCRA Facility Investigation Guidance, Chapter 5 (Data Management and Reporting) (EPA 530/SW-89-031, May 1989; OSWER Directive 9502.00-6D). Summaries of the data, including standard statistical properties, will be presented where applicable. An electronic data base and library of all data will be maintained, including QA/QC information and data validation packages, as backup to these summaries.

\section{COMMENT 152:}

Page 5-9, Section 5.4: This section should include tables indicating the formats for the following documents:

Preliminary Characterization Summary Reports and Preliminary Risk Analysis Reports.

\section{RESPONSE}

Agree.

COMMENT 153:

Page 5-11, Table 5-1: Add biota to Sections 2.1, 3.1, and 4.1. Note that Section 5.2.1 provides for the consideration of biological factors under the subheading "contaminant persistence".

\section{RESPONSE}

Agree.

\section{COMMENT 154:}

Page 5-14, Figure 5-3: This figure should be moved to a page occurring after the first reference to Figure 5-3 within the text. Also, the symbol representing the boundary for WAG-l should be deleted where it coexists with area streams to improve figure clarity.

\section{RESPONSE}

Section 5 was rewritten for Revision 1 of the RI Plan. Figure 5.3 was deleted from this section. 


\section{COMMENT 155:}

Page 5-15, Bullet 1: How is it intended to complete wells in each of the eight (8) stratigraphic units, by use of cluster wells or by single wells with multiple isolated intakes? The paragraph immediately below the bullets describes deep wells that only screen "the first water bearing fracture zone below the top of competent rock". Such wells will only screen one (1) stratigraphic unit at any point and not permit water quality comparisons in the units at a given location. Some clarification is necessary as to intent of the statement.

\section{RESPONSE}

Section 5 was rewritten for Revision 1 of the RI Plan. New well installation at WAG 1 is no longer a part of Phase I activities.

\section{COMMENT 156:}

Page 5-15, Para. 2: An evaluation of existing wells should be undertaken, and the results discussed in the text of the RI Plan and incorporated into the FSP.

\section{RESPONSE}

A well evaluation has been conducted. Results will be presented in a technical memorandum.

\section{COMMENT 157:}

Page 5-18, Para. 1: The results from the study conducted by Ketelle should be discussed in the text of the RI Plan and incorporated into the FSP.

\section{RESPONSE}

The following text is supplemental to Section 3.3.1.4:

During 1986 a north-south transect consisting of five coreholes ranging in depth from 116 to $143 \mathrm{~m}$, was installed at the ORNL Main Plant Area (Lee and Ketelle, 1988). Coreholes CH-1 through $\mathrm{CH}-5$ penetrate Units $A$ through $\mathrm{G}$ of the Chickamauga Group. (See Attachment to RCRA Part B, Response to Specific Comment 23 for locations). Examination of the cores revealed the Chickamauga units to be lithologically diverse, consisting of complexly interbedded limestone and siltstone lithofacies. Vertical and lateral lithologic repetition and interfingering are characteristic of nearly all units. The geophysical log suite run in these coreholes includes gamma, caliper, spontaneous potential, compensated density, neutron porosity, single point resistivity, long/short normal resistivity, temperature, and borehole deviation. The natural gamma logs show the interbedded nature within the units. Although indicating porosity, neutron porosity logs were not reliable indicators of groundwater flow 
zones. The relatively high porosity of siltstone- and shale-rich zones does not correlate to effective porosity for transmission of groundwater. The variability in long/short normal resistivity correlates to lithologic variability and mirrors changes in the gamma and neutron porosity traces. Geologic dip from rock uniformly measured 30 to $34^{\circ}$, consistent with values reported by Stockdale (1951). Small-scale structural features noted in the core include minor bedding plane fractures along thin lithologic partings, with evidence of high-angle fractures exhibiting little to no displacement.

To supplement hydrologic data from the coreholes, packer tests were performed on selected intervals. Derived hydraulic conductivity values in the coreholes ranged from $1 \mathrm{E}-4$ to $6 \mathrm{E}-9 \mathrm{~cm} / \mathrm{s}$.

The estimated static head distribution across the coreholes indicated significant artesian pressures at depth, with a general upward gradient. Average permeability increased south to north across the Chickamauga from the Rome Formation to the Knox Group. Decreasing permeability with depth or geologic unit was not evident. However, high heads appeared to correlate with low hydraulic conductivity. Discrete zones of higher permeability were noted and appeared bound by zones of relatively lower permeability. These zone were attributed to two factors: preferential weathering patterns along bedding planes in the various Chickamauga units and differential fracturing in response to historical deformation.

COMMENT 158:

Page 5-18, Section 5.1.1.7: It is not conclusive that no significant airborne pathway exists for contaminant transport based on the information in this Plan. The ORNL monitoring program and results should be thoroughly evaluated for precision, accuracy, representativeness and completeness, and be incorporated into the RI Plan.

\section{RESPONSE}

The air pathway will be assessed as part of the preliminary risk assessment process. The information from the ORNL monitoring program will be evaluated for potential incorporation into the remedial investigation.

\section{COMMENT 159:}

Page 5-18 Section 5.1.2 to Page 5-25: This information is already documented and should be deleted.

\section{RESPONSE}

Agree. 
COMMENT 160:

Pages $R-i$ to $R-l O$, References Section: The references should be modified to include current guidance documents.

RESPONSE

Agree.

COMMENT 161:

Page A1-2, Figure Al-l: This figure needs a scale.

RESPONSE

Agree.

COMMENT 162:

Page A1-5, Bullet Item 1: Need to also consider GPR for trench and pipeline location surveys. Also, need to consider seismic refraction and electrical resistivity surveys to define fracture zones and karst features in bedrock.

\section{RESPONSE}

See Responses to CERCLA Specific Comments 7 and 21 for detailed discussions of the applicability of geophysical methods to the WAG 1 RI.

COMMENT 163:

Page A1-5, Para. 1, Line 5: Replace the phrase "during subsequent phases" with "during RI Phase II activities".

\section{RESPONSE}

An iterative approach will be used to collect samples needed to satisfy the RI requirements. As data on the nature and extent of contamination become available from the first phase of sampling identified in the FSP, sampling efforts should narrow to a few significant areas where more intense sampling may occur during RI Phase II activities. 
COMMENT 164:

Page A1-6, Para. 1, Line 2: The results from the inactive tank sampling should be documented by the RI Plan.

\section{RESPONSE}

The results of the inactive tank sampling are included in Section 3.2 in Revision 1 of the RI Plan. References to the inactive tank sampling in A1.3 have been deleted. However, these tanks will be addressed in detail pursuant to Section IX of the proposed FFA.

\section{COMMENT 165:}

Page A2-1, Nondestructive Surveys Section: Need to also consider GPR for trench and pipeline location surveys. Also, need to consider seismic refraction and electrical resistivity surveys to define fracture zones and karst features in bedrock.

\section{RESPONSE}

See Responses to CERCLA Specific Comments 7 and 21 for detailed discussions of the applicability of geophysical methods to the WAG 1 RI.

\section{COMMENT 166:}

Page A2-4, Figure A2-1: This figure needs a scale.

\section{RESPONSE}

Agree.

\section{COMMENT 167:}

Page A2-5, Line 6: The USRADS operation procedures should be documented by the RI Plan.

\section{RESPONSE}

The following text is added as a supplement to the RI Plan:

Ultrasonic Ranging and Data Systems (USRADS) are swing instruments configured with ultrasonic and electronic equipment. A swing instrument consists of a hand-held meter, earphones, and a detector (sodium iodide) attached to the meter via a cord. The meter can 
alternatively read out in count rate or total count for fixed-time intervals. During gamma scanning, the gamma survey probe is moved slowly and is kept as close to the surface as possible. Any significant changes in gamma radiation levels above background, indicated by visual changes either in the instrument rate meter or the pitch of audio responses in the instrument headphones, are noted as reing anomalous. The ultrasonic and electronic equipment has two functions (1) to locate the instruments automatically with respect to a series transponders placed at known location at the site and (2) to transmit the data (radiation level and location measurement) to a microcomputer. While the data are being collected, the microcomputer displays a map of the site and the location of each data point on the map. This allows the technicians to determine whether they have completely covered the site. The data are stored electronically for on-site or later analysis. The USRADS survey protocol will include a walking rate of $0.5 \mathrm{~m} / \mathrm{s}$ (approximately $1.5 \mathrm{ft} / \mathrm{s}$ ) with a distance between parallel paths of $1.5 \mathrm{~m}(4.9 \mathrm{ft}$ ). The surveying instrument will be swung slowly from side to side as the surveyor moves forward. The instrument will undergo a daily calibration response check.

\section{COMMENT 168:}

Page A2-5, Section A2.3.1, Line 3: What the meaning of the phrase "transit paths?"

\section{RESPONSE}

The phrase "transit paths" means roads, sidewalks, and walking paths.

\section{COMMENT 169}

Page A2-5, Section A2.3.1, Line 3: What the meaning of the phrase "non-radiation workers.? Will the preliminary risk assessment consider the risk to "radiation workers" as well?

\section{RESPONSE}

The preliminary risk assessment considers only nonradiation workers. Nonradiation workers are maintenance workers doing routine passive activities (i.e., mowing; nonradiation, noninvasive activities).

\section{COMMENT 170:}

Page A2-7, Section A2.3.4, Line 2: After the phrase "human health" add "and the environment". 


\section{RESPONSE}

Agree.

COMMENT 171:

Page A2-7, Section A2.4, Para. 2, Line 1: Replace the term "EM-3" with "EM-31". RESPONSE

Agree.

COMMENT 172:

Page A2-7, Section A2.4: Need to discuss the interpretation limits of the EM-31 instrument. Also, need to describe the potential WAG-l-specific characteristics that may cause problems with EM-31 data quality.

\section{RESPONSE}

The EM conductivity method is a fast and inexpensive method to acquire ground conductivity measurements. The depth of investigation is about $3.1 \mathrm{~m}$ to $4.6 \mathrm{~m}(10.2$ to $15.1 \mathrm{ft})$ with resolution dependent upon the number and distance between sampling points. Buried trenches are often more conductive than undisturbed soils and can be located with this method. EM-31 data will be collected along lines collected in conjunction with the USRADS system. Features in the SWSA and Waste Pile areas that may affect the data quality include fences and overhead utilities. It is anticipated that quality data can be collected at a distance of up to $6.1 \mathrm{~m}(20.0 \mathrm{ft})$ from these structures. See Response to RCRA Part A General Comment 5 for additional information.

COMMENT 173:

Page A2-8, Figure A2-2: This figure needs a scale.

\section{RESPONSE}

Agree. 


\section{COMMENT 174:}

Page A2-9, Section A2.5: Need to discuss the interpretation limits of the XRF instrument. Also, need to describe the potential WAG-1-specific characteristics that may cause problems with XRF data quality.

\section{RESPONSE}

XRF measurements are made using a field-portable XRF instrument, which uses a radioactive source and gas proportional tube detector to measure element-specific energy emissions.

Depending on the matrix being analyzed, measurements may be made in situ or on minimally processed samples. Calibrating with matrix samples from the location to be sampled generally allows detection limits around $100 \mathrm{ppm}$ for many metals with no sample preparation, if the in situ soil is finer that an 80 mesh sieve. Minimal sample preparation such as air drying and sieving the samples (100 to 200 mesh sieve) improves detection limits if the same techniques were used in calibration. X-ray fluorescence is a useful technique for some situations.

While XRF could conceivably be applied in several ways (e.g., for field screening of split spoon samples to guide sample selection for laboratory analysis), it is not planned for use during Phase I investigations. It is felt that its detection limits are too high and that it would provide false negatives if used to define areas of contamination. Generally speaking, analytical results in the tens-of-parts per million range can be acquired in less than a minute of counting.

\section{COMMENT 175:}

Page A3-1, Section A3.1: Need to provide a discussion in the RI Plan concerning the preliminary investigation of groundwater flow and quality at WAG-1, and the FSP should reference the RI Plan.

\section{RESPONSE}

A discussion of the groundwater flow conceptual model is provided in Response to CERCLA Specific Comment 98. A table of groundwater quality parameters from a scoping survey is provided in the Attachment B, Part A, Response to RCRA Specific Comment 20.

DOE agrees that the FSP should reference the RI Plan.

\section{COMMENT 176:}

Pages A3-1 to A3-16, Sections A3.2 to A3.7: Each tasks or objectives subsection should specify how the investigation is expected to contribute to the objectives stated in Section A3.1, Page A3-1. 


\section{RESPONSE}

Each section noted already contains statements that relate how the task will help to meet the stated objectives of the groundwater investigation.

\section{COMMENT 177:}

Page A3-2, Figure A3-1: This figure needs a scale.

\section{RESPONSE}

Agree.

\section{COMMENT 178:}

Page A3-3, Section A3.3.1, Para. 1, Line 5: This section reports that "up to 50 stainless steel compliance wells" will be evaluated. However, Figure A3-1 only indicated that 42 wells currently exist. How will the extra 8 wells be accounted for during the RI? Also, the results from the well and piezometer headspace gas analysis survey should be submitted for review and comment before the final selection of wells to be sampled during RI Phase I activity, and the construction details (e.g., depth of screened interval) of wells proposed for sampling should be provided.

\section{RESPONSE}

At the time the RI Plan was written, 42 wells existed in the ORNL Main Plant Area. The phrase "up to 50 ... wells" was used because it was anticipated that additional wells might be installed. Two wells (Monitoring Wells 946 and 947) subsequently have been added near SWSA 1.

The headspace gas analysis is a field activity (Section A3.2) being carried out under the groundwater investigation (Section A3.0). The results of the activity are currently being reviewed and evaluated. The data will be reported in a technical memorandum. Screen depths and water level measurements will be included in that document.

\section{COMMENT 179:}

Page A3-10, Para. 3, Line 1: After the phrase "radioactive constituents. add ", except tritium,".

\section{RESPONSE}

Agree. 


\section{COMMENT 180:}

This section should provide for pumping tests for those well pairs screened in the water table aquifer and the first confined hydrologic unit below the water table aquifer to define vertical hydrological communication properties.

\section{RESPONSE}

The execution of pumping tests are not planned for Phase I scoping activities. Better information is needed on local flow patterns and the nature and extent of contamination before the artificial stress of pumping tests are applied. They will be considered in Phase II, if appropriate.

\section{COMMENT 181:}

Page A3-13, Section A3.6.1, Para. 2: The task concerning the evaluation of existing deep corehole data should be performed and the results incorporated into the RI Plan.

\section{RESPONSE}

Agree.

\section{COMMENT 182:}

Page A4-1, Section A4.1, Para. 1, Line 5: Replace the phrase "a significant exposure pathway or component of an exposure pathway for human exposure to contaminants" with "a significant pathway or component of a pathway for human exposure to hazardous substances, and a pathway for adverse environmental impacts".

\section{RESPONSE}

Agree.

\section{COMMENT 183:}

Page A4-3, Para. 2, Line 1: Need to document the nsasimum Cs-137 concentration found within the contaminated area shown on Figure A4-1, including the maximum concentration found in the flrodplain located downstream. 


\section{RESPONSE}

A sediment sample was analyzed by ORNL on a multichannel analyzer. ${ }^{137} \mathrm{Cs}$ was found to be the cause of elevated readings, but the concentration was not reported. The source area, which appears to be a seep, is located on First Creek south of White Oak Avenue. The floodplain downstream of the seep also has shown elevated radiological readings. Contamination in that area is thought to be the result of backwater from WOC flooding.

\section{COMMENT 184:}

Page A9-2, Table A9-1: For volatile and mobile radionuclides, e.g., tritium and carbon-14, a separate sample bottle should be used and filled without air space. A preservative (base, e.g., $\mathrm{NaOH}$ ) should be used for samples to be analyzed for tritium. Groundwater samples should be collected with and without filtration for comparison, and holding times should depend on isotope half-life.

\section{RESPONSE}

Agree, with the exception that no preservative will be used during sampling for tritium. $\mathrm{NaOH}$ is added at the laboratory during sample preparation. The holding time for water samples to be analyzed for tritium will be 45 days. Sampling of water for tritium analysis without preservation is in conformance with EPA Method 906.0 for drinking water sampling. 
RCRA GENERAL COMMENTS

\title{
RESPONSE TO RCRA COMMENTS ON WAG 1 RI PLAN
}

\author{
PART A - GENERAL COMMENTS
}

\section{INTRODUCTION}

The RCRA General Comments issued by EPA's Region IV (Atlanta, Georgia) office on April 7, 1989, were based on Revision 0 of the RI Plan dated December 1987. These comments include issues raised by the Tennessee Department of Conservation (formerly TDHE). The responses in the following paragraphs address each of the comments. Supporting documentition referenced in the responses is contained in Attachment B, Part A. Supporting documentation appears in the attachment in the same order as the responses appear in the following paragraphs.

\section{COMMENT 1:}

The proposed monitoring well network may not be adequate to evaluate whether each solid waste management unit (SWMU) is contributing contaminants to the ground water (Section A7.3). For the evaluation of 99 SWMUs, this plan proposes 35 new monitoring wells. The locations of these new wells have not been selected. These new wells are in addition to approximately 40 existing monitoring wells. However, these existing wells may not all be downgradient of SWMUs. Some existing wells may not be in working order.

\section{RESPONSE:}

The original RI Plan (December 1987) was inadequate to evaluate the degree and extent of contamination. However, this document is no longer relevant; Revision 1, dated August 1989, and the proposed Oak Ridge FFA established major modifications to the original plan.

A phased strategy is being used to investigate the potential contamination from the 99 SWMUs. Under Phase I, groundwater flow patterns will be determined in conjunction with groundwater quality characterization. In general, existing wells and piezometers will be used for this characterization. The full nature and extent of contamination will be refined under Phase II. There is an additional set of SWMUs recently identified in the proposed FFA. The need for further, detailed investigations of these SWMUs under Phase I is currently being evaluated. Field investigations and sampling will be performed if determined to be necessary at the conclusion of this evaluation.

Under Phase I, 46 existing wells and 48 piezometers will be sampled under low-base, low-base storm event, high-base, and high-base storm event conditions. The 48 piezometers were selected 
for upgrading from a population of approximately 100 piezometers. Piezometers that exhibited signs of surface water infiltration, collapse, or clogging were rejected for upgrading.

These wells and piezometers, plus an additional 63 piezometers, are also monitored monthly for water level data. Continuous water level measurement stations will be installed at 22 wells. These data will be used to construct groundwater flow nets and flow models; these will advance the interpretation of groundwater quality sampling data and support the development of sampling strategies for Phase II.

In addition to these existing wells and upgraded piezometers, 13 new RCRA-quality monitoring wells will be sampled to provide background groundwater quality data.

A number of SWMUs are clustered together, such as underground tank farms within a common pit. Due to the close proximity of these units, it is not always necessary to treat each SWMU individually during Phase I of the investigation. Groundwater quality can be asse;ssed using wells, piezometers, SWMU content sampling data, and new soil borings located 'within the downgradient portions of such pits, thereby minimizing unnecessary hazards of Irilling within a group of closely spaced buried tanks of undetermined structural integrity.

Phase I investigations also will include completion of approximately seven bedrock coreholes, each to a potential depth of $152.4 \mathrm{~m}$ (500 feet). This coring activity is designed to determine groundwater flow patterns in the deep, fractured bedrock beneath the site. These coreholes, plus an additional existing three holes will be tested using a packer-transducer assembly to determine hydraulic conductivities and pressure gradients. Water samples will be withdrawn from selected zones. Geophysical logs will be collected from each of the new coreholes, and analyzed in conjunction with the logs from the existing holes. The new and existing coreholes will provide north-south transects of hydrogeologic conditions along two important hydraulic boundaries adjacent to WAG 1 (First Creek and Fifth Creek) and along the central highland within WAG 1 (Third Street).

In addition to these activities, Phase I also includes an analysis of the extent to which underground pipeline trenches serve as conduits for contaminant migration. Soil samples from approximately 51 borings will be collected from pipeline trenches closely associated with SWMUs identified as leak/spill sites. Fourteen additional locations were selected where trenches may serve as shallow migration pathways for collection of upgradient SWMUs and spill locations. Soil samples from approximately 230 additional locations associated with leak and spill sites, SWMUs, and areas of elevated radiations levels identified from the USRADS survey will also be collected. This network of sampling locations will provide a thorough area coverage for characterizing groundwater quality under the Phase I objectives. 


\section{COMMENT 2:}

Slug tests and dye tracer tests are proposed to evaluate the rates and directions of ground-water flow at this site (Section A7.6). In addition, these tests are also proposed to evaluate the flow between and through the different formations at the site and through a fault near the site. These methods may not be adequate for all of the hydrologic uncertainties at this site. For example, some of the formations are shales. Dye tracer tests may require too much time to be useful to evaluate the hydrologic properties of the shales. Also, where the geologic conditions will permit an aquifer test with a pumping well and several observation wells, such aquifer tests will be useful in defining the anisotropy of the aquifer. This may be a useful technique at this site since there are already a large number of piezometers.

\section{RESPONSE:}

DOE agrees with the utility of dye and tracer tests, as well as the limitations of these tests in dense shales. This comment is no longer relevant to Revision 1 of the RI Plan, dated August 1989. In recognition of the limitations of dye and tracer tests, packer tests and geophysical logging in the deep coreholes will be used to characterize hydrogeologic properties of the shale units.

Tracer tests have been previously used at ORNL to evaluate the potential nature and extent of underground pipeline leaks. Data for these studies are being used under Phase I of the RI investigation. Further dye and tracer tests will be considered under Phase II.

Pump tests to determine aquifer properties will not be performed under Phase I. These tests pose a risk of introducing contamination, in response to artificial hydraulic gradients, into previously uncontaminated areas. There will not be enough data to assess this risk until Phase I studies are completed. Pump tests are also limited by the nature of the low permeability soils and rock present at ORNL. Pump tests will be considered under Phase II if it is determined that they can provide meaningful data without risk of spreading contamination.

\section{COMMENT 3:}

The parameters proposed for analysis of contaminated soils are not adequate to determine the impact of contaminated soil upon the ground water (Section A5.4.1). In some cases the contaminant of concern is not proposed for analysis. One example of this involves the four mercury spill sites. The deep soil samples at these sites are not proposed for mercury analysis (SWMU 1.1, 1.2, 1.3, 1.4). Also, one important measurement of the ability of soils to leach contaminants, the distribution coefficient, is not proposed to be measured at this site. 


\section{RESPONSE:}

Investigations of SWMUs will include analysis of compounds characteristic of each SWMU. Mercury will be included in sampling each of the mercury spill sites. In fact, mercury is included in the target analyte list for metals for all samples collected at WAG 1. In addition, approximately 100 distribution coefficient determinations will be made for soil/sediment samples.

\section{COMMENT 4:}

The TCL is not sufficient as a screening list. Unless all possible RCRA constituents have been identified that can be present at the site. The screening list should be Appendix VIII or IX for each SWMU or group of SWMUs that received identical wastes.

\section{RESPONSE:}

Phase I of the investigation will include analysis of Appendix IX compounds from approximately 15 to 20 percent of the groundwater and surface water samples. These data will be used to refine future analyte lists.

\section{COMMENT 5:}

The geophysical surveys proposed for SWSA 1 and SWSA 2 may not be adequate for this geologic setting. The purpose of these surveys is to locate trenches with unknown waste contents. Two geophysical methods are proposed: ground penetrating radar and magnetometer surveys. The ground penetrating radar is unlikely to work because of the high clay content of the soils. The magnetometer surveys will not be useful for locating waste in pasteboard and wood boxes.

\section{RESPONSE:}

Ground penetrating radar (GPR) will not be used, due to the factors cited, as provided in Revision 1 of the RI Plan. In place of magnetometer surveys, the EM-31 method will be used to evaluate the potential for buried metallic objects such as drums at SWSA 1 and 2. While it is possible that clay-rich soils may limit the effectiveness of this method, there is little alternative to the use of this method. There are no remote sensing methods that can directly locate pasteboard and wood boxes.

To supplement the EM-31 data, detailed measurements of surficial radiation levels will be made using USRADS. This method provides approximately 63 data points per square meter (3600 per acre); data on dose and radioactive disintegrations per minute are collected. These data will be used to assess the potential for surface and near-surface radioactive contamination. Results will be used in designing sampling plans. 


\section{COMMENT 6:}

The RIP should contain a schedule of the specific investigatory activities that are to be conducted including dates for task initiation and completion of sampling and analysis activities as well as duration of each activity. The timing and scheduling concerns are important because inputs and outputs of the various sampling and analytical activities are connected to other investigative activities within the overall RFI study timeline.

\section{RESPONSE:}

The schedule for completing the WAG 1 RI Plan has recently been addressed in the proposed FFA, but has not been agreed to by all parties (EPA and TDC). These schedules are presently being re-negotiated. To ensure that milestones are met, the project is being managed using the Cost/Schedule Control System, used for other large DOE projects. The specific dates for task initiation and completion, and the interrelationship of each task, are established within this system. Critical path activities are easily identified and tracked over the life of the overall project. The figure included in Attachment B, Part A - RCRA General Comments, Response to General Comment 6 shows the overall schedule for Phase I activities. 


\title{
RESPONSE TO RCRA COMMENTS ON WAG 1 RI PLAN
}

\author{
PART A - SPECIFIC COMMENTS
}

\section{INTRODUCTION}

The Part A - RCRA Specific Comments issued by EPA were based on Revision 0 of the RI Plan dated December 1987. The responses in the following paragraphs address each of the comments. Supporting documentation referenced in the responses is contained in Attachment B, Part A RCRA Specific Comments. Supporting documentation appears in the Attachment in the same order as the responses appear in the following paragraphs.

\section{COMMENT 7:}

Page 1-5 - One missing technical issue the extent of groundwater contamination.

\section{RESPONSE:}

The existing groundwater well network (monitoring wells and piezometers) is being used during Phase I, where possible, to define the nature, distribution, and movement of contaminants in groundwater. Additional wells may be installed under Phase II.

\section{COMMENT 8:}

Page 2-11 - What are the fourth and fifth levels of reporting?

\section{RESPONSE:}

This section discusses administrative cost and scheduling and administrative controls under the work breakdown structure. It does not refer to reporting the detection of environmental contamination.

\section{COMMENT 9:}

Page 3-6 and Figure 3-2 - A stratigraphic column is given, not a geologic cross section as stated in the RIP. Also, the Ft. Payne "chart" should probably be "chert." 
PART A - RCRA SPECIFIC COMMENTS

\section{RESPONSE:}

This stratigraphic column was erroneously referred to as a geologic cross section. The Ft. Payne is a chert.

\section{COMMENT 10:}

Page 3-32 to 3-47 - In the section on waste storage tanks, there is not adequate information for most SWMU tanks. In order to evaluate these tanks EPA will need to know what kind of tanks these are (e.g. aboveground, onground, inground or underground) to evaluate this document. Requirements for monitoring will vary by tank type.

\section{RESPONSE:}

The tanks, in general, in WAG 1 that have been designated SWMUs are buried tanks. The collection and storage tank SWMUs fall into three categories: inactive LLW tanks, active LLW tanks, and sewage and chemical waste tanks. These classifications are explained in Revision 1 of the RI Work Plan and are now further addressed in the proposed FFA.

COMMENT 11:

Page 3-33 to 3-42 - 101 SWMUs are listed yet this document states that there are 99. Please clarify this discrepancy.

RESPONSE:

There are 99 SWMUs listed in Table 3-10. SWMU 1.5 itself is not an individual SWMU and should not be counted. SWMU $1.23 \mathrm{~b}$ was listed twice.

COMMENT 12:

Page 3-48 - The North and South Tank Farms and isotope production areas are not labeled on Figure 3-5. Instead, Figure 3-5 depicts the endangered species existing in the area of WAG $l$.

\section{RESPONSE:}

Figure 3-5 describes the endangered species near WAG 1. The figure that should have been referenced is Figure 3-6, Map of WAG 1 Showing Location of SWMUs. The North and South Tank Farms are at the intersection of Central Avenue and Third Street. The Isotope Production Area is northwest of the intersection of Central Avenue and Fifth Street. 


\section{COMMENT 13:}

Page 3-63 and Table 3-11 - The units of the Chickamauga Group with solution channels which are referred to in the text should be reflected in the description of the units in the table.

\section{RESPONSE:}

The units that Stockdale (1951) considers susceptible to the development of solution channeling are Units $\mathbf{G}$ and $\mathbf{H}$. The table referred to above is taken directly from Stockdale and has not been modified. The first sentence of this paragraph should read as follows: "The upper limestone units of the Chickamauga Group, Units $\mathrm{G}$ and $\mathrm{H}$, are described as being tightly cemented and compact, with the exception of several small solution channels, typically around $2.5 \mathrm{~cm}$ but sometimes as large as $30.5 \mathrm{~cm}$ in diameter (Stockdale, 1951)."

\section{COMMENT 14:}

Page 3-63 - Second hand information is mentioned in the text. "It is reported that..." This report should be obtained and incorporated into the work plan so that data gaps can be identified.

\section{RTESPONSE:}

The information available at the time the RI Plan was written was via verbal communication. Since that time the original document, Lee and Ketelle (1988), has been obtained. See Part A RCRA Specific Comments, Response to Specific Comment 44 for discussion of this report. Geophysical logs included gamma, caliper, spontaneous potential, compensated density, neutron porosity, single point resistivity, and long/short normal resistivity. Initial observations of the cores and geophysical logs indicated that the rock is tightly cemented and competent. Fractures often appear to be remineralized with calcite and many exhibit signs of motion.

\section{COMMENT 15:}

Figure 3-9 - Which way does this cross section trend? What is the scale of this cross section?

\section{RESPONSE:}

The cross section trends north (left) to south (right). Although this cross section is not strictly to scale and is intended to illustrate generalized geology, a rule of thumb scale would be 1:4800 for the map provided with the document. The cross section represents a vertical relief of approximately $23 \mathrm{~m}(75 \mathrm{ft})$. 


\section{COMMENT 16:}

Table 3-12 - A table of soil cores is presented at some sites. It would be helpful to have map showing the locations of these cores. Also, the SWMU numbers for each core would be helpful on this list.

\section{RESPONSE:}

Figure 3-9A was added to Revision 1 of the RI Plan (August 1989) and includes the location of these sites as well as other soil investigations. The original soil investigations were done prior to the development of the SWMU concept. The Preliminary Site Characterization Summary Report and Soil Technical Memorandum will relate previous investigations to SWMUs.

\section{COMMENT 17:}

Page 3-88 - The reports referenced in this section, Stockdale (1951), Webster (1976), and Ketelle et al. (1985), should be included in ait appendix since important conclusions are drawn from these reports.

\section{RESPONSE:}

The above referenced documents will be provided under separate cover letter.

\section{COMMENT 18:}

Page 3-89 - The reference to a report by Ketelle et al. (1985) concerning a reduction in flow during pumping tests of two wells should be clarified. In addition, this section discusses a "further investigation" of these two supply wells but does not provide the details to support any conclusions or provide a reference.

\section{RESPONSE:}

The supply wells that were mentioned in the above reference were older wells located

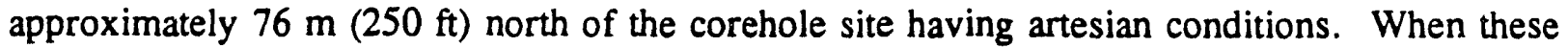
wells were being pumped, hydraulic head in the corehole was affected. Details of these wells are contained in a letter from Dr. Wm. McMaster to Dr. D.J. Nelson, Ecological Research, ORNL. The phrase "Further investigation" referred to securing this document, not to further field work to obtain details of the wells. 
PART A - RCRA SPECIFIC COMMENTS

\section{COMMENT 19:}

Figure 3-19 - It is stated that not all piezometer data was used Why? Also, a plot of the locations of the piezometers used would be helpful.

\section{RESPONSE:}

This figure is part of the Ketelle et al. (1986) document. Although the statement that not all piezometer data was used appears on the figure, no indication is given in this document of the reason for the omission of piezometer data. Although the piezometer locations were not included in this figure, Figure A3-1 of the Field Sampling Plan of the RI Plan, dated August 1989, is a map showing piezometer locations as well as compliance well locations.

\section{COMMENT 20:}

Page 3-92 - It is stated that groundwater monitoring occurred in 1985 and 1986. The data from each monitoring well should be included in this document. It could provide useful information about the extent of contamination and could help prioritize the locations of additional wells. Also, there is a statement that groundwater samples were obtained from piezometers that are constructed from PVC. Since the contaminants of concern at some of the sites are organics, $P V C$ is not an appropriate well construction material since it may interact with organics.

\section{RESPONSE:}

The data for individual piezometers are included in Attachment B, Part A - RCRA Specific Comments, Response to Specific Comment 20.

Although PVC piezometers may not be appropriate for sampling organics, the main contaminants of concern within WAG 1 are radioactive. There is no apparent interference between PVC and radioactive elements that would negate the use of the piezometers in this Phase I investigation. The piezometers, properly developed and upgraded, will provide locations for opportunistic sampling in Phase I.

The data from the complianse wells surrounding the impoundments were obtained from the yearly environmental surveillance document published by ORNL. The data are in summary form. Data on individual wells will be utilized in compiling the Preliminary Site Characterization Summary Report and Preliminary Risk Assessment Report. 
COMMENT 21:

Table 3-16 - The chemical parameters included in the contaminant scoping study apparently did not include some contaminants of concern. For example mercury and PCBs are not on this list.

\section{RESPONSE:}

The contaminant scoping survey performed by ORNL apparently did not include mercury or PCBs. Groundwater sampling during the RI will include analyse; of these contaminants.

\section{COMMENT 22:}

Table 3-17 - MCLs for Carbon Tetrachloride, l,l-Dicholoroethene, and Trichloroethene have been exceeded at this site. Since no well specific information is provided, it is provided, it is not clear how extensive this contamination is. Also, why were two compounds of concern measured in only one well?

\section{RESPONSE:}

The extent of contamination evidenced by this data will be assessed and utilized in compiling the Preliminary Site Characterization Summary Report and Preliminary Risk Assessment Report. It is unknown why acetone and carbon disulfide were measured in only one well.

\section{COMMENT 23:}

Table 3-38 - The values for the detection limits of Toxaphene are above the practical quantitation limit in the 7-7-87 Federal Register notice. Since the MCLG for Toxaphene is 0 the detection limits should be at the practical quantitation limit. In addition, at least one well had a pH of 13. Wells with high pH values should be identified.

\section{RESPONSE:}

The Phase I RI will follow the EPA Contract Laboratory Program (CLP) procedures and protocols. The possibility that some wells have very high or very low $\mathrm{pH}$ is being assessed and the findings will be reported in future reports such as technical memorandum or the Preliminary Site Characterization Summary Report. The elevated detection levels cited for toxaphene might have been attributable to sample dilutions by the laboratory. This issue will be examined in preparing the Preliminary Site Characterization Summary Report. 
COMMENT 24:

Table 3-19 - There was still detection of contamination even at these high detection limits. Once again, the detection limits for Toxaphene were set above the practical quantitation limits.

\section{RESPONSE:}

As stated above, the Phase I RI will follow CLP procedures and protocols. Analyses will include those for the presence of toxaphene. Please refer to Part A - RCRA Specific Comments, Response to Specific Comment 23.

\section{COMMENT 25:}

Page 4-7 - The exposure assessment appears to be incomplete since a scenario does not exist to investigate the potential impact of contamination upon ORNL employees. Also, the off site exposure assessment should consider all pathways, not just the one with the greatest potential.

\section{RESPONSE:}

The exposure scenarios in Section 4 were revised after the RI Plan, Revision 0, was issued. Several pathways will be included in the preliminary risk assessment, including ORNL employees as receptors.

\section{COMMENT 26:}

Table 4-6 - This table is incomplete for groundwater. Other issues include the effect of anisotropy on groundwater at the site, the hydrologic interactions of the residual soil, the bedrock formations and the fault, and the effect of nearby supply wells and the sump in the Oak Ridge Reactor Building on ground-water flow.

\section{RESPONSE:}

The groundwater system as well as soils and geology will be fully investigated to better describe contaminant movement. This investigation will also aid in the evaluation of remedial alternatives.

\section{COMMENT 27:}

Page 5-3 - It is stated that the initial radiological analysis will be for the radiological contaminants already suspected. Then analysis for additional compounds will be conducted if warranted. How will ORNL know if the additional analysis is warranted? 


\section{RESPONSES:}

These statements were omitted from the RI Plan, Revision 1 (August 1989). The presence of all contaminants will be investigated and samples will undergo a full suite of analyses. Not only will radioactivity analyses of gross alpha and gross beta be performed, but specific isotopics will be analyzed if the gross alpha and gross beta screens suggest the presence of radioactive contaminants.

\section{COMMENT 28:}

Page 5-5 - It is stated that the sediments of the surface impoundments have been sampled and that no further sampling is required during this phase of the RI. The results of this sampling should be included in the RI plan or referenced so that it may be reviewed for adequacy.

\section{RESPONSE:}

Section 3.2.3 was revised for Revision 1 of the RI Plan (August 1989). Radiological sampling in the Waste Holding Basin (SWMU 1.12) indicated the presence of ${ }^{60} \mathrm{Co},{ }^{137} \mathrm{Cs},{ }^{90} \mathrm{Sr},{ }^{154} \mathrm{Eu}$, ${ }^{238} \mathrm{Pu},{ }^{239} \mathrm{Pu},{ }^{241} \mathrm{Am}$, and ${ }^{244} \mathrm{Cm}$ in sediment samples and the presence of ${ }^{137} \mathrm{Cs}$ and ${ }^{90} \mathrm{Sr}$ in surface water. Chemical sampling indicated the presence of PCBs in the surface water and mercury in sediments. Sludge analysis from the Equalization Basin (SWMU 1.13) indicated the presence of ${ }^{137} \mathrm{Cs},{ }^{90} \mathrm{Sr}$, Th, U, and TRU wastes. In the Process Waste Ponds 3539 and 3540 , analyses of sludge samples indicated that extraction procedure (EP) toxicity limits were not exceeded. The average activities of ${ }^{90} \mathrm{Sr},{ }^{238} \mathrm{Pu}$, and ${ }^{239} \mathrm{Pu}$ were high in soils at the Low Intensity Test Reactor (LITR) Pond (SWMU 1.19) site. The Sewage Aeration Ponds 2543 and 2544 apparently have not been sampled. Sludges from the sewage impoundments will be sampled as part of the RI.

\section{COMMENT 29:}

Page 5-6, Sect. 5.1.1.4 - States that the objectives of the soil sampling is to, "verify the existence and/or type of contamination at suspected sites (e.g., SWMUs) and to determine the type and average concentration of contaminants at worst sites." The objective of the RFI should go well beyond verification and determination of the average concentration of contaminants. The scope of the RFI should include defining the extent of soils contamination as well as characterization of the contamination. The workplan should include provisions for accomplishing this objective.

\section{RESPONSE:}

The purpose of soil sampling during Phase I of the RI is to gather and analyze data necessary to perform a preliminary assessment of risk to human health and environment and to begin to 
develop and assess remedial alternatives. The Phase I investigation will contribute greatly to an understanding of the nature and extent of contamination through investigation of soils associated with solid waste management units. Characterization of the nature and extent of contamination will be further refined during the Phase II operable unit-specific investigations.

\section{COMMENT 30:}

Page 5-7 - The radiological surface scan will only be useful for indications of radioactive soils. The reviewer is concerned that the walkover rad survey meant to locate radiation anomalies and prioritize sampling will not define areas contaminated with non rad contaminants. Does documentation exist that indicates that areas contaminated with nonradiation contaminates correlate with radioactive contaminants to the extent that defining radioactive soils will serve to encompass nonrad areas?

\section{RESPONSE:}

Information from the USRADS walkover survey is being utilized to pinpoint areas of suspected surface radioactive contamination. Approximately 40 soil boring sites out of 300 identified to date are the result of the radiation walkover survey. Most SWMUs are associated with leak and spill sites and are documented to have contained various types of radioactive components. While the majority of the remaining borings are associated with these SWMUs, borings are planned in areas of known chemical and other non-rad contamination. For example, at least 12 soil borings are designed to investigate known mercury spills. All samples collected undergo screening for volatile organics at the CSL.

\section{COMMENT 31:}

Page 5-8 and subsequent pages - Compositing soil samples may allow volatile contaminants to escape. Grab samples are recommended.

\section{RESPONSE:}

Soil core samples are composited over $0.6-\mathrm{m}(2.0-\mathrm{ft})$ intervals, not $1.5-\mathrm{m}(5-\mathrm{ft})$ intervals as originally proposed. As soon as the split spoon sampler is opened and scanned by the health physics specialist, the volatile organics sample is collected by the sample technician by scraping soil directly into the sample container. The remainder of the soil is then composited and other sample containers filled. Sampling in this manner minimizes the escape of volatile contaminants. 


\section{COMMENT 32:}

Page 5-8 - The locations of the proposed surface to bedrock soil samples should be provided for evaluation.

\section{RESPONSE:}

Generally, all soil borings are advanced to auger refusal, or bedrock, with the exception of some borings which must be hand augered because of limitation to access by heavy drilling equipment.

\section{COMMENT 33:}

Page 5-9 - Soil samples may not be helpful in locating groundwater contamination plumes. This will require groundwater monitoring wells.

\section{RESPONSE:}

During the Phase I investigation, 46 monitoring wells and 48 piezometers have been sampled under low-base and high-base flow conditions to ascertain the presence/absence of radioactive components, chemical contaminants and inorganic parameters. Soil samples do, however, provide data on the intermedia transfer potential of contamination.

\section{COMMENT 34:}

Page 5-9 - Soil samples should also be tested to determine porosity, organic carbon content, clay content, and the distribution coefficients for contaminants.

\section{RESPONSE:}

Geotechnical parameters for approximately 100 samples will be determined during Phase I.

\section{COMMENT 35:}

\section{Page 5-9. Section 5.1.1.4 - Unbiased sampling should proceed until the full extent of contamination is determined.}

\section{RESPONSE:}

Several sampling strategies are being used during Phase I. Biased samples are being collected to both confirm and assess the magnitude of releases from SWMUs. This includes most soil samples and groundwater samples collected near SWMUs. Opportunistic samples are being 
collected from 48 upgraded piezometers distributed throughout WAG 1. This will provide information upon which operable unit-specific characterization strategies can be implemented during Phase II. Both biased and unbiased samples are being collected from surface waters. Biased samples will be taken from below outfalls and seeps associated with individual SWMUs, while unbiased samples will be collected at various locations along White Oak Creek and its tributaries. Background soil, groundwater, and surface water quality samples will also be collected.

Unbiased sampling only during Phase $\mathrm{I}$ is inappropriate given the need to characterize releases from individual SWMUs.

\section{COMMENT 36:}

Page 5-10 - This page is missing from the copy reviewed - cannot evaluate.

\section{RESPONSE:}

Page 5-10 in Revision 0 of the RI Plan contained Figure 5-2, Unbiased Sampling Protocol Illustration. Section 5 was extensively rewritten for inclusion in Revision 1 (August 1989). This figure no longer is applicable.

\section{COMMENT 37:}

Page 5-12, Sect. 5.1.1.6 - Indicates that BNI will review existing data and determine data needs, and also as a result of review, tasks described in $R I$ plan may be revised., Why was data not reviewed before hand to determine needs. Seems logical that since purpose of $R I$ is to determine extent and character of contamination and need for corrective measures, a preliminary step to preparing an investigative workplan is to review data to determike needs. How can EPA approve a workplan that indicates that the investigations specified may not be those actually performed?

\section{RESPONSE:}

Since preparation of the December 1987 RI Plan, more than 100 reports have been reviewed. Information presented in these reports serves as the framework for the Phase I investigations. Additional reports will be synthesized into the study, as they become available. 
COMMENT 38:

Page 5-12 - The results of the tests on the inactive impoundment should be included in this document to justify no additional sampling in this $R I$ phase.

\section{RESPONSE:}

See Part A - RCRA Specific Comments, Response to Specific Comment 28, for discussion of sampling results for the inactive impoundments.

COMMENT 39:

Page 5-13 - Additional important factors that may influence groundwater flow at this site include hydraulic conductivity and heterogeneity and anisotropy.

\section{RESPONSE:}

These factors will be assessed during the Phase I investigation.

COMMENT 40:

Page 5-13 - The construction details of existing wells should be provided.

\section{RESPONSE:}

This information will be collected and reported in the Preliminary Site Characterization Summary Report. The 27 RCRA compliance wells installed by ORNL were designed in accordance with RCRA guidance.

\section{COMMENT 41:}

Page 5-15 - The specific methodology for the ground-water testing should be provided including the literature citation.

\section{RESPONSE:}

Groundwater testing such as pump tests and tracer testing will be considered for use in the operable unit-based Phase II investigation. Methodologies for these tests will be included in the Phase II Work Plan. 


\section{COMMENT 42:}

Page 5-16. Section 5.1.1.6 - What will be the criteria for well selection? Where will new wells, be installed, if contamination is found, will additional wells be installed to define plumes. This should be discussed in workplan or is this part of the "data gaps" repeatedly referred to in workplan. This should be part of objective of groundwater monitoring.

\section{RESPONSE:}

The Phase I investigation has collected groundwater samples from the existing monitoring well network. In addition, 48 piezometers have been upgraded to furnish groundwater samples in the interior of WAG 1. Except for 13 background quality wells, no new wells will be installed during the Phase I investigation. The need for additional wel!s will be evaluated. It is expected that new wells, if needed, will be installed during the Phase II investigation.

\section{COMMENT 43:}

Page 5-17. Sect. 5.1.1.6 - The workplan refers to the possible employment of tracer test without offering any specifics. Expand on the tracer test discussion. Detail the rationale for their use, how they will be carried out, and specify the procedures. The discussion should address, but not be limited to:

a. Specific dyes to be used and criteria for selection.

b. Proposed dye injection points or criteria for their selection.

c. Type of tracing (qualitative or quantitative).

d. Method of dye detection and location of detectors or sampling points or the criteria for selection.

e. Sample times or interval.

f. How long will sampling continue or how long will detectors be left in place if the dye does not come through immediately.

\section{RESPONSE:}

Tracer tests, if needed, will be executed during the Phase II investigation. Details and methods employed for these tests will be included in the Phase II Work Plan.

COMMENT 44:

Page 5-18 - A second hand reference is provided. ORNL should obtain the study by Ketelle rather than attempt to describe what is reported to have been said. 


\section{RESPONSE:}

The reference for this report is: Lee R. R. and R. H. Ketelle, Subsurface Geology of the Chickamauga Group at Oak Ridge National Laboratory, ORNL/TM-10749, Oak Ridge National Laboratory, 1988. The following is a summary of the study and supplements the text of the RI Plan:

A north-south transect consisting of five coreholes ranging in depth from 116 to $143 \mathrm{~m}$ (380.6 to $469.2 \mathrm{ft}$ ), was installed at the ORNL Main Plant Area along Fifth Street. Coreholes CH-1 through $\mathrm{CH}-5$ penetrate Units A through $\mathrm{G}$ of the Chickamauga Group. Examination of the cores revealed the Chickamauga units to be lithologically diverse, consisting of complexly interbedded limestone and siltstone lithofacies. Vertical and lateral lithologic repetition and interfingering are characteristic of nearly all units. The geophysical log suite run in these coreholes includes gamma, caliper, spontaneous potential, compensated density, neutron porosity, single point resistivity, long/short normal resistivity, temperature, and borehole deviation. The natural gamma logs show the interbedded nature within the units. While indicating the porosity distribution within the Chickamauga Group, neutron porosity logs were not reliable indicators of groundwater flow zones. The relatively high porosity of siltstone- and shale-rich zones does not correlate to effective porosity for transmission of groundwater. The variability in long/short normal resistivity correlates to lithologic variability and mirrors changes in the gamma and neutron porosity traces. Geologic dip from rock uniformly measured 30 to 34 degrees, consistent with values reported by Stockdale (1951). Small-scalc irructural features noted in the core include minor bedding plane fractures along thin lithologic partings, with evidence of high-angle fractures exhibiting little or no displacement.

\section{COMMENT 45:}

Page 5-19. Section 5.1.3.2 - Indicates that sampling necessary to characterize nature, concentration, and volume of contaminants in each inactive tank will be performed by energy systems in accordance with protocols established by ORNL and reviewed by BNI. Does this mean that the type and extent of sampling will be determined after approval of the $R I$ workplan. Again, the object of the workplan is to identify and define investigative procedures and therefore should be included within the workplan not established later.

\section{RESPONSE:}

Sampling of the inactive tank contents has been addressed separately by the proposed FFA and, therefore, is being performed separately from the RI. Data from these investigations are being used in the Phase I study to assess releases from these tanks. 


\section{COMMENT 46:}

Page 5-21 - Raw data is to be analyzed according to techniques presented in Section 5.2. However, no specific techniques were presented in this section.

\section{RESPONSE:}

Raw data will be analyzed with available statistical techniques to derive useful correlations, trends, averages, etc. Standard statistical methods will provide statistical, graphical, and reporting capabilities. The SAS system will be used to calculate the arithmetic mean and the population standard deviation. Methods and references in RAGS Risk Assessment Guidance for Superfund, Volume 1, (EPA, 1989) Human Health Evaluation Manual (Part A) will be used to estimate the upper confidence limit (i.e., the 95 percent upper confidence limit on the arithmetic mean).

\section{COMMENT 47:}

Page A1-8 - This page indicates that 99 SWMUs are located in WAG 1. Table 3-1 lists 101 SWMUs.

\section{RESPONSE:}

Table 3-10 lists the 99 SWMUs that were named at the time this report was written. See the Part A-RCRA Specific Comments, Response to Specific Comment 11, for clarification of the number of SWMUs in this table.

\section{COMMENT 48:}

Page A1-12, Table A1-3 - Table A1-3: No analyses of mercury, a contaminant of concern, is proposed for any "unbiased" soil samples or any ground-water samples. Also, the measurement of the distribution coefficient, organic carbon content, clay content, and porosity are recommended for soil samples. Additional parameters may be required where metals are contaminants of concern. These include conductivity, total dissolved solids, hardness, density, dissolved oxygen, alkalinity, sulfate, chloride, nitrate, ammonia, ortho phosphate, heavy metals, iron, manganese, sodium, potassium, calcium, magnesium, bicarbonate, carbonate, silica, and sulfides and [hydrogen sulfide or methane] where samples have zero dissolved oxygen and an H2S odor. If metals are present, measurements should be made of $\mathrm{Fe}+2$, $\mathrm{Mn}+2, \mathrm{Cr}+6$ and $\mathrm{Cr}+3$.

\section{RESPONSE:}


Table A1-3 in Revision 0 of the RI Plan did not include analyses for mercury or miscellaneous parameters. This section of the Field Sampling Plan was extensively rewritten in Revision 1 (August 1989), separating discussion of soil and groundwater samples. Both the soil and groundwater sampling plans in Revision 1 include full metal analyses as well as analyses of miscellaneous parameters.

\section{COMMENT 49:}

Page A4-1 - Provide details of how fractioning of the sediment samples collected from the creek channels into three particle-size ranges will be accomplished while preventing alteration of true contaminant concentrations in the sediments.

\section{RESPONSE:}

Creek sediment samples are not separated into size fractions, but are collected as grab samples and placed directly into the sample containers. This method minimized loss or alteration of contaminants contained in the sediments. The original plan to size fraction was in response to a study by ORNL researchers that suggested that contaminants selectively adsorbed onto the gravelsize particles. If the Phase I sampling effort demonstrates that contaminants are present in the stream sediments, a more detailed sampling plan will be proposed in the Phase II Field Sampling Plan.

\section{COMMENT 50:}

Page A5-9. Table A5 - The proposed "C" samples are composite samples from several boreholes. Composites may mask contaminated areas and is not recommended. For SWMUs 1.1 to 1.4 no analysis for mercury in the deep samples is proposed, yet mercury is a coitaminant of concern at this site. Also at these same SWMUs no analysis is proposed for gamma spectrometry, alpha emitting isotopes, or in the shallow samples. For SWMUs 1.5a-w, a group of 46 deep samples are proposed. The number of samples for each SWMU should be specified. Mercury is of concern at some of these sites yet no mercury samples are proposed. On SWMU 1.6 the depth for sample number 4 is missing. Since SWMU 1.7 may have involved stack emissions, should some samples be taken at a shallow depth? Since SWMU 1.8 involved a surface spill, should some of the samples be at a shallow depth? SWMU 1.9 is an underground tank. Where are these samples in relation to the depth of the tank? For SWMU 1.11, a waste holding basin, why are only deep samples taken? For SWMUs 1.12, 1.13, 1.14, 1.16, and 1.17 why are the samples all at the edge or outside the unit? Also, for SWMUs $1.12,1.13,1.14,1.15,1.16,1.17,1.18$ and 1.19, which are surface impoundments, why are only deep samples proposed? For SWMU 1.20 acids were used to wash filters at this site. What acids were used and are they part of the proposed analysis? For SWMUs 1.21, 1.44, $1 A .1,1 A .5,1 A .11,1 A .12,1 A .13,1 A .14,1 A .15$, and $1 A .16$ no soil analyses are proposed. How is this justified? For SWMU 1.22 two samples are proposed for underground ductwork. 
Is this adequate? SWMU 1.23 is two gunite tanks. One soil sample is proposed for the analysis of these tanks. Similar samples are proposed for the other tanks (SWMUs 1.23 to 1.43 and SWMU 1.45). It is not possible to evaluate the adequacy of these samples since almost no information on these tanks was provided. SWMUs 1.46 and 1.47 are burial grounds. Since no information exists for the waste placed in these areas, the analysis should be more comprehensive than just gross alpha, gross beta, gamma spectroscopy, alpha emitting isotopes, and beta emitting isotopes as is proposed for many of the samples. For example, an analysis for mercury, which is of concern at other sites, is not proposed in any of the samples from these sites. In SWMU 1.49, the neutralization facility, why are the analyses for the shallow samples limited to radioisotope analyses? In SWSA 1.50, the PCB storage area, an analysis for PCBs is needed. SWMUs LA.l to $1 A .16$ are not on the list of SWMUs in Table 3.10.

\section{RESPONSE:}

This section of the Field Sampling Plan was substantially revised in Revisicn 1 (August 1989). Analyses of soils associated with SWMUs include not only analyses of compounds characteristic of the SWMU but routinely include analyses for radioactive isotopes, volatile and semi-volatile organics, and metals. The strategy prescribed in Revision 1 will address the cited concerns.

\section{COMMENT 51:}

Page A5-18. Figure A5-2 - This summary of the soil analysis should contain distribution coefficient determination, organic carbon content, clay content, and porosity.

\section{RESPONSE:}

Table A6-2 replaces Table A5-2 in Revision 1 of the Field Sampling Plan dated August 1989. Analyses of samples will include determination of appropriate physical and geochemical properties.

\section{COMMENT 52:}

Page A5-19 - It is indicated that the locations of the soil sampling points may be changed significantly. ORNL should decide where they want to sample and include this in the final RI work plan.

\section{RESPONSE:}

Revision 1 of the RI Plan explains the sampling strategy, in response to this concern. 


\section{COMMENT 53:}

Page A5-20 to A5-24, Figures A5-2a to A5-2e - The locations of the proposed samples are depicted on these figures. Since the RI work plan does not include site specific information about the location of contamination in the soil or ground-water of the details of the design of these SWMUs it is not possible to evaluate these selected locations.

\section{RESPONSE:}

The coordinates and locations for leak/spill sites, now SWMUs, are contained in the draft copy of the ORNL Contaminated Site Summary Sheets document dated September 30, 1990. Soil sampling sites in Phase I of the remedial investigation will be placed downgradient and along strike of the suspected leak/spill site in order to determine the presence of contaminants.

\section{COMMENT 54:}

Page A5-26 - How will the soils sampled in half of the monitoring wells be selected?

\section{RESPONSE:}

After the initial issuance of the Field Sampling Plan, the decision was made to use the existing monitoring well network in the groundwater investigation. Therefore, this section no longer applies.

\section{COMMENT 55:}

Page A5-29 - The process of selecting the limits of the surface composite soil samples is discussed. The process of compositing these samples may result in some areas of contamination not being detected either by dilution or by loss of volatile constituents. Grab samples are recommended.

\section{RESPONSE:}

Areal compositing is not being performed in the Phase I investigation.

\section{COMMENT 56:}

Page A5-29 - This report refers to a DOE manual as the source for the soil sampling methodology. DOE should provide this manual as an appendix. 


\section{RESPONSE:}

This comment is no longer applicable. As discussed in Revision 1 of the RI Plan, areal composites are not being collected.

\section{COMMENT 57:}

Page A5-30 - The reference for soil compositing procedures in Section 4.6.3.3.4 of the EPA SOP and $Q A$ Minual is actually in the section on streambed sampling.

\section{RESPONSE:}

Section 4.9.4 Sampling Techniques for soil samples in the EPA SOP and QA Manual states that "mixing of the soil sample for chemical analysis should be performed in accordance with the procedures outlined in Section 4.6.3.3.4."

\section{COMMENT 58:}

Page A5-31 - The procedures for archiving samples should be part of this document.

\section{RESPONSE:}

Specific archiving procedures are contained in Project Procedure 1605, "Sample Archiving." This procedure is included in Attachment B, Part A-RCRA Specific Comments, Response to Specific Comment 58.

\section{COMMENT 59:}

Page A7-1 - An important parameter for groundwater at this site may be the amount of recharge. It is recommended that ORNL conduct a water balance study of this area. Also, what will the field evaluation of existing wells involve? How will the subsurface configuration of the well be determined?

\section{RESPONSE:}

A water balance study is being performed as part of the Phase I investigation. These data will be included in the Groundwater Technical Memorandum. Field evaluation of existing wells and piezometers included performing a headspace gas analysis to test for the presence of volatile organic components, determining construction materials and suitability for upgrading, and 
evaluating the location in relation to known areas of contamination. Subsurface construction was determined from existing ORNL documents detailing well and piezometer installation.

COMMENT 60:

Page A7-2 - What is the scale on this map?

RESPONSE:

The scale of the map provided with the original issuance of the RI Plan is 1:4800.

COMMENT 61:

Page A7-4 - The well screen size should be determined by site specific sieve analysis.

\section{RESPONSE:}

The wells are screened in either clay-rich overburden or fractured limestone. Sieve analyses would be inappropriate for use in this setting. The smallest practical screen size is selected and a compatible sand is used.

\section{COMMENT 62:}

Page A7-5 - The rationalization for the selection of the monitoring well locations is referenced to Section 5.1.1.7 which is titled Rationale for Air Sampling.

RESPONSE:

Reference should have been made to Section 5.1.1.6.

\section{COMMENT 63:}

Page A7-5 - The project procedure on well installation should be included in this RI plan.

\section{RESPONSE:}

This comment no longer applies to Revision 1 of the RI Plan. Stainless steel wells are installed in accordance with EPA/RCRA guidelines. 


\section{COMMENT 64:}

Tables A7-1 and A7-2 - The screen size should be determined by sieve analysis. PVC riser should not be used where organics are suspected. The sand pack should extend approximately 2 feet above the screen.

\section{RESPONSE:}

See the Part A-RCRA Specific Comments, Response to Comment 61 , for discussion of screen size determination. Use of PVC risers may be inappropriate for sampling some organics. See Part A - RCRA Specific Comments, Response to Specific Comment 20, for discussion. The sandpack will extend $0.61 \mathrm{~m}(2 \mathrm{ft})$ above the screen in the wells.

\section{COMMENT 65:}

Page A7-9 - How will the fracture zones in the bedrock be located?

\section{RESPONSE:}

Fracture zones are to be identified by coring the bedrock and visually examining the rock core. Downhole geophysical logging will also be used to identify fracture zones.

\section{COMMENT 66:}

Page A7-10 - Clarify the sentence which reads, "Parts of the sandpack not filled with sand or bentonite will be filled with cement/bentonite grout."

\section{RESPONSE:}

The sentence should read "The annular space above the sandpack and bentonite seal will be backfilled with cement/bentonite grout."

\section{COMMENT 67:}

Page A7-10 - The sand size in the sand pack should be selected to be compatible with the formation after sieve analysis. Sand should extend at least two feet above the screen. The bentonite seal should extend at least two feet above the sand pack.

\section{RESPONSE:}

Shallow overburden wells are screened in clays. Sieve analyses are inappropriate for this type formation. Instead, the smallest practical screen size was selected and a sand compatible with 


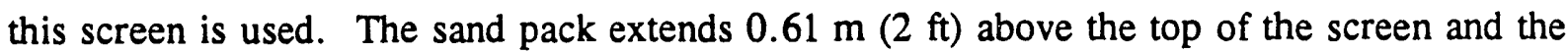
bentonite seal is $0.61 \mathrm{~m}(2 \mathrm{ft})$ above the top of the sand pack.

\section{COMMENT 69:}

Page A7-10 to A7-11 - When developing the well, after the first surge with a surge block, pump the well to remove the sediment in the water. Also, do not worry about the air-lift pump forcing air into the formation. In addition, ten well volumes will probably not be adequate to develop this well. The development should proceed until the water becomes sediment free.

\section{RESPONSE:}

Because the wells are screened in clay-rich overburden, multiple attempts at using a surge block have not resulted in sediment-free water. The procedure for development does not depend on the number of well volumes but recommends development until the water is sediment free and parameters ( $\mathrm{pH}$, temperatures, conductivity) have stabilized. In most cases, 10 well volumes are not adequate. Historically in Bethel Valley, it has not been possible to achieve sediment-free water in wells screened in overburden, and the decision has been made to rely on developing the well for a reasonable amount of time and achieving stable water quality parameters.

\section{COMMENT 69:}

Page A7-12 - This indicated that sampling locations will be selected on the basis of criteria in section A7.2. However, this section is on the evaluation and upgrade of existing wel's not sampling. Also, ORNL should propose sampling wells based on an evaluation of the existing data. Specific wells for sampling should be proposed in the work plan.

\section{RESPONSE:}

This section was rewritten in Revision 1 of the Field Sampling Plan (August 1989). During the Phase I investigation 48 piezometers were upgraded within WAG 1 . Selection of these wells for upgrade was based on proximity to areas of suspected contamination and an evaluation of piezometer construction. These 48 piezometers and the existing 46 monitoring wells were sampled under low- and high-base flow conditions, as well as during storm events within each condition.

\section{COMMENT 70:}

Page A7-12 - Where the monitor wells are high yield, the wells should be purged before sampling until $\mathrm{pH}$ and specific conductivity stabilize. Where the monitoring wells are low yield, the procedures in Practical Guide for Ground-Water Sampling (EPA/600/2-85/104) are recommended. 


\section{RESPONSE:}

The groundwater sampling efforts in WAG 1 are being conducted in accordance with the Environmental Surveillance Procedures of Martin Marietta Energy Systems (MMES 1987) which are consistent with the document cited above. These procedures have been approved by DOE and are being utilized in conjunction with the QAPP and event-specific field work guides.

\section{COMMENT 71;}

Page A7-16 to A7-18 - The methods for aquifer testing should be specified. This should be accompanied by a citation from the literature for each method.

\section{RESPONSE:}

Aquifer testing is not being performed during the Phase I investigation. The section on aquifer testing was omitted in Revision 1 of the Field Sampling Plan. Should Phase I groundwater investigations indicate the need for aquifer testing, the methods will be included in the Phase II Work Plan.

\section{COMMENT 72:}

Attachment A-4 - Several beta emitters with half lives longer than days or hours from page 331 (list of known contaminants at this site) are missing from this list of radiological constituents. These are C-14, Ni-63, Pm-147, U-232, Np-237, and Cm-244.

\section{RESPONSE:}

Samples which indicate the presence of radioisotopes through gross alpha and gross beta screens will undergo isotopic analyses.

\section{COMMENT 73:}

Page B5-2 Where an analytical scheme is used that will result in the elimination of samples from further analysis it is recommended that some samples which appear uncontaminated pass through to higher levels to ensure that the system is working.

\section{RESPONSE:}

All collected surface and groundwater samples are screened at the onsite CSL; the CSL also screens all soil samples. Approximately 50 percent of subsurface soil samples and all surface soil samples are sent forward by the onsite laboratory for analysis based on screening data. 
Approximately 5 percent of the subsurface soil samples that have levels below the screening criteria will be sent forward to the offsite laboratory for further analysis.

\section{COMMENT 74:}

Page B9-6 - This page indicates that analysis for cyanide and PCBs during the RI investigation may be discontinued at the discretion of the WAG manager. These contaminants should be part of the analysis at any unit where they are of concern.

\section{RESPONSE:}

Attachment B following the Field Sampling Plan in Revision 0 was rewritten and included in Revision 1 of the Field Sampling Plan as Section 10. Samples from locations suspected to contain PCB or cyanide contamination will be analyzed for those components. 


\section{RESPONSE TO RCRA COMMENTS ON WAG 1 RI PLAN}

\section{PART B - SPECIFIC COMMENTS}

\section{INTRODUCTION}

The Part B - RCRA Specific Comments issued by EPA were based on an advance draft copy of the Field Sampling Plan of Revision 1 of the RI Plan. The firal text of Revision 1 was issued in Auglist 1989. The responses in the following paragraphs address each of the comments. Supportive documentation referenced in the responses is contained in Attachment B, Part B RCRA Specific Comments. Supporting documentation appears in the attachment in the same order as the responses appear in the following paragraphs.

\section{COMMENT 1:}

Page A1-1. There was no Figure A1-1 included in this submittal.

\section{RESPONSE}

Figure A1-1, WAG 1 Site Map, is included in Revision 1 to the WAG 1 RI Plan (August 1989). All missing fi gures and/or tables are included in the August 1989 revision. This responds to Part B-RCRA Specific Comments 3, 8, 11, 18, 25, 29, and 42.

\section{COMMENT 2:}

Page A1-5. Using x-ray fluorescence to determine the presence of metals is of limited value, considering the high detection limits and the fact that results are not very repeatable.

\section{RESPONSE:}

While X-ray fluorescence (XRF) may be used to determine the presence of heavy metal constituents in soils and sediment, the technique is only appropriate for metals under certain favorable combinations of gross contamination and matrix. After careful consideration of the circumstances which are known to affect its performance, XRF is not currently being used in the WAG 1 RI. However, XRF will continue to be considered if laboratory analyses of samples from an area show sufficiently high concentrations of metals to make the use of XRF effective. 


\section{COMMENT 3:}

Page A1-7. The Attachment A-2 referenced in this table was not included in this submittal.

\section{RESPONSE:}

All missing figures and/or tables are included in Revision 1 of the RI Plan (August 1989).

\section{COMMENT 4:}

Page A1-8. The CSL will probably not be adequate to identify indicator parameters; a Level IV laboratory is recommended.

\section{RESPONSE:}

The CSL is used for screening samples for radioactivity and volatile organic compounds (VOCs). Radiological analyses being performed are gross measurements for alpha and beta activity, tritium, and gamma spectroscopy. Gamma spectroscopy is used whenever samples are approximately at the transportation limit for limited quantity radioactive materials shipments, and for further isotopic information for health and safety and RI purposes. Typical detection limits for radiological analyses are as follows:

$$
\text { Soil, } \mu \mathrm{Ci} / \mathrm{g} \quad \text { Water } \mu \mathrm{Ci} / \mathrm{mL}
$$

$\begin{array}{lll}\text { Gross Alpha } & 1.3 \mathrm{E}-5 & 2.0 \mathrm{E}-8 \\ \text { Gross Beta } & 2.1 \mathrm{E}-5 & 3.0 \mathrm{E}-8 \\ \text { Tritium } & 1.1 \mathrm{E}-6 & 1.2 \mathrm{E}-6\end{array}$

Gamma Spectroscopy (Dependent upon isotope and other factors)

Gas chromatography (GC) determinations consist of analyzing gases or headspace from water or soil samples. A laboratory GC with photoionization and electrolytic conductivity detectors has been installed, allowing for the detection of halogenated and double-bonded organic compounds. The system has been set up and optimized for the identification and quantitation of a predetermined list of VOCs. Tentative identification of other compounds not on the predetermined list is also performed. Detection limits are:

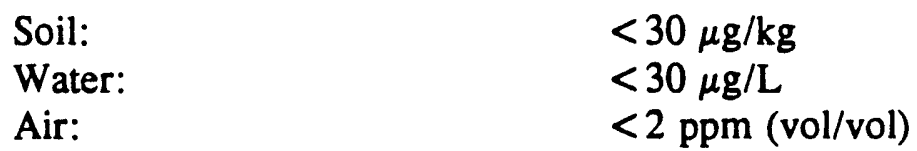

All analyses are performed using industry-accepted methods or manufacturers' instructions. A Quality Assurance Plan has been developed for the CSL, including quality control procedures for radiological and GC analyses. The data generated by the CSL are Level 1. 
Following screening, the data are reviewed for presence of radionuclides and VOCs. If a sample has levels significantly above background, the sample is sent to the laboratory for various analyses such as the TCL list and the full suite of radiological analyses. This does not preclude sending samples in which no hazardous constituents were detected by field screening.

\section{COMMENT 5:}

Page A2-2. Explain "...defined locations."

\section{RESPONSE:}

"Defined locrtions" refers to locations of anomalously high surface scan count rates. Anomalously high count rates are identified by two methods: 1) preparing a contour map or track map (color coded to count rates) of the data to identify peaks, and/or 2) by sorting the data to identify readings where high scan count rates (above threshold) but low dose rates (below threshold) are encountered. For this task, thresholds are set at 18,000 counts per minute (cpm) and $100 \mathrm{uR} / \mathrm{hr}$. These thresholds are roughly 3 to 4 times the background radiation levels measured during the walkover survey.

The walkover survey consists of a surface scan and discrete measurements using a cone shield at the locations of anomalously high surface scan count rates. The cone shield is used to segregate readings resulting from ground contamination from air scatter radiation which may influence the surface scan measurements. The same type detector (Nal) used in the surface scan is used with the cone shield.

\section{COMMENT 6:}

Page A2-3. There was no Figure A2-1 included in this submittal.

The instructions for the walkover survey could not be evaluated since Project Procedure 1209 was not included for review.

\section{RESPONSE:}

All missing figures and/or tables are included in Revision 1 of the RI Plan (August 1989).

Project Procedure 1220, Initial Site Survey, is included in Attachment B, Part B - RCRA Specific Comments, Response to Specific Comment 6. 


\section{COMMENT 7:}

Page A2-5. Procedures for the USRADS system could not be evaluated since they were not included in this submittal.

\section{RESPONSE:}

The procedures used in the USRADS survey are included in Attachment B, Part B - RCRA Specific Comments, Response to Specific Comment 7.

\section{COMMENT 8:}

Page A2-7. There was no Figure A2-2 included in this submittal.

\section{RESPONSE:}

All missing figures and/or tables are included in the August 1989 revision.

\section{COMMENT 9:}

Page A2-9. How was the grid for the EM-31 survey determined?

The section on $x$-ray fluorescence (XRF) is somewhat misleading. More than minimal sample preparation is required for meaningful results; samples should be dried and ground to a fine powder. For best results, calibration should be performed using standards in the matrix to be analyzed; non-homogeneity of soils on the site would cause serious accuracy problems. The detection limits of the instrument are such that its use is limited to samples with high concentrations of some metals.

\section{RESPONSE:}

The EM-31 survey grid was oriented perpendicular to the suspected trenches in SWSA 2. Since the SWSA 2 trenches probably ran east-west by the ORNL grid system, it was convenient to orient the grid system for the EM-31 and magnetometer surveys for all the areas surveyed parallel to the existing ORNL grid system. The grid spacing has been reduced to $3.0 \mathrm{~m}(10 \mathrm{ft})$ for better coverage.

After careful consideration of the circumstances which are known to affect its performance, XRF is not currently being used in the WAG 1 investigation. However, XRF is a useful technique for some situations. 
Many of its capabilities and limitations have recently been documented allowing effective application to situations where it is of benefit. Therefore, XRF will continue to be considered if laboratory analyses of samples from an area show sufficiently high concentrations of metals to make the use of XRF effective.

Depending on the matrix being analyzed, XRF measurements may be made in situ or on minimally processed samples. Calibrating with matrix samples from the location to be sampled generally allows detection limits around $100 \mathrm{ppm}$ for many metals with no sample preparation, provided the in situ soil is finer than an 80 mesh sieve. Minimal sample preparation such as air drying and sieving the samples through 100 - or 200 -mesh sieve improves detection limits provided the same techniques were used in calibration.

While XRF could conceivably be applied in several ways (e.g., for field screening of split spoon samples to guide sample selection for laboratory analysis), its limitations restrict its use during Phase I. There is too great a risk that it could provide false negatives. If areas of high concentrations of metal contaminants are delineated using the traditional methods, XRF may be considered during Phase II to further characterize such zones.

\section{COMMENT 10:}

Page A2-10. "The water table is believed to be..." This should be a known factor by now and not an assumption.

\section{RESPONSE:}

The water table is shallow enough to intersect the building basements in the area south of Central Avenue.

\section{COMMENT 11:}

Page A3-1. There was no Figure A3-1 included in this submittal.

\section{RESPONSE:}

All missing figures and/or tables are included in Revision 1 of the RI Plan (August 1989).

\section{COMMENT 12:}

Page A3-3. What is the purpose of sampling gas from the top of the well bore? This will only give some idea of what gas(es) are volatizing from the top of the water column, not the entire water column or the surrounding soil. 


\section{RESPONSE:}

Headspace analysis is a screening tool which is used to rapidly identify areas of possible organic contamination. However, because of the limitations of the method and the potential for false negatives, the method alone is not used to eliminate areas from consideration.

Due to the extensive network of piezometers throughout WAG 1 (roughly 100), this technique can be particularly informative as a gross screening tool. Headspace gas sampling is not intended to look at the entire water column. Actual sampling of the well and samples of soil adjacent to the well would be needed to identify layering of contaminants.

\section{COMMENT 13:}

Page A3-3. Piezometers are used primarily for water level measurements and are not usually constructed in the same manner as monitoring wells that will be used to collect groundwater samples. What data supports the premise that piezometers at this facility have been constructed and maintained adequately to be used for sampling purposes?

\section{RESPONSE:}

The piezometers are of PVC construction and were installed using procedures to avoid crosscontamination of holes. The extensive network of piezometers throughout WAG 1 provides an excellent opportunity to obtain water quality data, provided the piezometers are adequate for Phase I sampling purposes and the limitations of the data are understood. PVC piezometers that are of good construction and have been properly developed may be adequate for sampling many analytes, including radioisotopes. Data concerning analytes which may be influenced by well construction, such as VOCs, are viewed in that context.

It is not cost effective to install new RCRA-standard teflon or stainless steel monitoring wells until the scoping opportunities afforded by the existing piezometer network have been utilized. RCRA-standard wells will be installed during Phase II at data-critical locations.

The specifications used for piezometer installation are available and were reviewed. Piezometers were also evaluated in the field. Forty-eight piezometers selected for sampling were upgraded, as required, and developed in accordance with Martin Marietta Energy System Procedure ESP 600. As a minimum, upgrading consisted of installing guard posts, locking well caps and new concrete pads to protect the annular and surface seals of the piezometers.

\section{COMMENT 14:}

Page A3-4. Assuming that compliance wells have been adequately developed may lead to erroneous data. This should be confirmed in the field. 


\section{RESPONSE:}

The compliance wells were installed and developed using procedures and specifications designed to meet EPA requirements then in effect for water quality wells. Documentation is available and was reviewed as necessary to confirm the quality of the wells. No compliance wells were identified which require development.

\section{COMMENT 15:}

Page A3-6. Wells should be developed until turbidity is reduced and pH, temperature, and specific conductivity stabili:e.

ESP-600 and ESP-900 referenced here were not included for review; therefore, decon procedures, etc., could not be evaluated.

\section{RESPONSE:}

Wells are developed in accordance with Martin Marietta Energy Systems Procedure ESP 600, which requires that wells be developed until turbidity is reduced and $\mathrm{pH}$, temperature, and specific conductivity stabilize. ESP-900 is included in Attachment B, Part B - RCRA Specific Comments, Response to Specific Comment 20.

\section{COMMENT 16:}

Page A3-7. Piezometer data gathered in 1986 being used-is more recent data available? Also, piezometers are generally used for collecting water level measurement only. Why were these used to collect water samples when monitoring wells should have been available? Samples for dissolved metals should also be taken for comparison purposes. The fact that the samples taken from the piezometers were so turbid supports the contention that the piezometers were installed for the collection of water levels, not for collecting water quality samples.

\section{RESPONSE:}

Piezometer data, other than water levels, more recent than 1986 are not available. As part of the Phase I investigation, 48 piezometers have been sampled for TCL, TAL, radiological constituents, and TDS. The samples were also analyzed for total and dissolved concentrations of metals and radiologic constituents. The piezometers were installed throughout WAG 1 as a prelude to any water quality well installation, whereas the majority of the water quality wells were installed at the WAG perimeter to evaluate transport pathways off-WAG. Screening type analyses of groundwater samples collected from piezometers can be used to intelligently site any additionally needed water quality wells. Useful water quality data can be obtained from properly constructed and developed piezometers provided the limitations of the data are known. 
Please refer to the Part B-RCRA Specific Comments, Response for Specific Comment 13, for further information.

Samples of both dissolved (filtered) and total (unfiltered) metals are being collected for comparison. In addition, a set of piezometer samples will be analyzed for "Total Recoverable Metals", which will provide an indication of the metallic contamination sorbed onto native clay and silt particles present in the water sample. These results will provide an understanding of the influences of turbidity.

\section{COMMENT 17:}

Page A3-8. What is the purpose of collecting groundwater samples during high and low water tables?

\section{RESPONSE:}

Sampling during the seasonal high and low water tables, as well as during storm events during high and low water table conditions, is designed to represent times when the contaminated materials are in maximum and minimum contact, respectively, with the groundwater. Since the majority of contaminant transport in WAG 1 has been hypothesized to be associated with storm events, it is necessary to define potential pathways during both water table conditions.

\section{COMMENT 18:}

Page A3-9. Attachments A-2 and A-3 alluded to in this table were not included in this submittal.

\section{RESPONSE:}

All missing figures and/or tables were included in Revision 1 of the RI Plan (August 1989).

\section{COMMENT 19:}

Page A3-10. Lowering the pump intake to within a few inches of the well bottom may stir up sediment or silt. What steps will be taken to alleviate this problem?

If samples are collected for filtered dissolved metals samples, then samples should also be collected for total metals samples. 


\section{RESPONSE:}

In wells with dedicated sampling pumps, samples are collected with the pump at its present depth. In wells sampled using a bladder pump decontaminated between wells, the pump intake is lowered to within several inches of the bottom of the screen. Prior to sampling, each well is purged in accordance with EPA protocol. If the well was constructed with a sump and properly developed, excessive sediments are typically not a problem. For those wells without sufficient sumps, care is taken to minimize stirring up excessive sediment.

Non-filtered and filtered samples are being collected for total and dissolved metals analyses, respectively.

\section{COMMENT 20:}

Page A3-10. Procedure ESP-900 was not included for review; therefore, decon procedures could not be evaluated.

\section{RESPONSE:}

Sampling equipment decontamination practices are based on standard EPA methods in use for the RCRA and CERCLA programs, as well as radiation-specific procedures in use at ORNL.

Equipment rinseate samples are collected during field sampling to ensure that decon practices are effective. ESP-900 is included in Attachment B, Part B - RCRA Specific Comments, Response to Specific Comment 20.

\section{COMMENT 21:}

Page A3-12. What factors will determine which four week period water levels will be collected?

\section{RESPONSE:}

Monthly water levels are being collected at approximately 160 locations throughout the WAG 1 area. Water level measurements will be collected at each of these stations for up to one year. In addition to monthly measurements, continuous water level monitors will be installed at 22 of those locations. The starting point of the 4-week cycle was a function of the availability of field personnel.

\section{COMMENT 22:}

Page A3-13. Please define "...unknown geophysical logging." 


\section{RESPONSE:}

When the RI Plan was written, data were not available concerning the type of geophysical logging performed on the coreholes previously installed at the site. The word "unknown" should be deleted.

\section{COMMENT 23:}

Page A3-14. Figure A3-2 was illegible.

\section{RESPONSE:}

The corrected figure is included in Attachment B, Part B - RCRA Specific Comments, Response to Specific Comment 23.

\section{COMMENT 24:}

Page A4-16. The exact permeability testing procedures were not included for review.

\section{RESPONSE:}

In situ tests for hydraulic conductivity will be used. Specific field procedures will be developed using EPA methods (e.g., SW-846 Method 9100) where appropriate.

\section{COMMENT 25:}

Page A4-3. Figure A4-1 was not included in this submittal.

\section{RESPONSE:}

All missing figures and/or tables are included in Revision 1 of the RI Plan (August 1989).

\section{COMMENT 26:}

Page A4-5. What factors determined these transects? 


\section{RESPONSE:}

The transects were devised only to provide sampling points across the floodplain. A radiologic walkover survey was executed to verify that no significant radiologically contaminated spots were missed. As a result, one additional transect was sampled.

\section{COMMENT 27:}

Page A4-6. Section B9.2.1 of the QAPP was not included for review; therefore, the "rationale" referenced here could not be evaluated.

The ESD SOP also recommends using a Pyrex container when multiple aliquots are taken.

\section{RESPONSE:}

The Field Sampling Plan, Revision 1, moved information contained in Section B9.2.1 to Section A10.2.1. The full suite of TCL compounds and TAL metals, plus tritium, gross alpha, gross beta, gamma spec, and specific isotopes will be analyzed. Martin Marietta Energy Systems Procedure No. 308-1, "Composite Procedures," (MMES 1987), recommends a stainless steel bowl for compositing multiple aliquots.

\section{COMMENT 28:}

Page A5-4. The DOE report referenced here was not included; therefore, the "Additional specific guidance" could not be reviewed.

\section{RESPONSE:}

This comment is no longer relevant to Revision 1 of the RI Plan. Surface water sampling equipment has been selected using standard EPA guidance (e.g., Superfund Compendium of Field Operations Methods or RCRA Facility Investigation Guidance).

\section{COMMENT 29:}

Page A6-1. Figure A6-1 was not included in this submittal.

\section{RESPONSE:}

All missing figures and/or tables are included in Revision 1 of the RI Plan (August 1989). 


\section{COMMENT 30:}

Page A6-4. The methodology used to determine these number of soil samples per area size was not explained in the text.

\section{RESPONSE:}

The number of soil samples is based on a professional evaluation of waste/unit characteristics, release mechanisms, and environmental setting. Sample collection in Phase I is biased toward determining the nature and general magnitude of releases from SWMUs. During Phase II, the overall extent of these releases will be determined. Thus, specific criteria for sample density per unit area are not relevant to Phase I. Criteria will be developed for operable unit-specific work plans for Phase II.

\section{COMMENT 31:}

Page A6-7. The DOE report referenced here was not included; therefore, procedures for soil sampling could not be fully evaluated.

\section{RESPONSE:}

This comment is no longer applicable. Soil sampling methods are taken from standard EPA protocols provided in the RCRA Facility Investigation Guidance, the Superfund Compendium of Field Operations Methods, and ORNL's ESPs.

\section{COMMENT 32:}

Page A6-9. The DOE report referenced here was not included for review.

\section{RESPONSE:}

This comment is no longer applicable. Drilling and sampling methods are taken from standard EPA protocols provided in the RCRA Facility Investigations Guidance, the Superfund Compendium of Field Operations Methods, and ORNL's ESPs.

\section{COMMENT 33:}

Page A7-1. Method ESP-301-1 referenced here was not included and could not be evaluated for adequacy. 


\section{RESPONSE:}

Method ESP 301-1 is included in Attachment B, Part B - RCRA Specific Comments, Response to Specific Comment 33.

\section{COMMENT 34:}

Page A7-2. Method ESP-303 referenced here was not included for review.

\section{RESPONSE:}

Methods ESP 303-4 and ESP 303-6 are included in Attachment B, Part B - RCRA Specific Comments, Response to Specific Comment 34.

\section{COMMENT 35:}

\section{Page A7-3. Why aren't the same water quality analyses being performed each quarter?}

\section{RESPONSE:}

Water samples were collected from each of the background monitoring wells, selected WAG 1 (i.e., not background) monitoring wells and upgraded piezometers in the fall of 1990 and analyzed for Appendix IX constituents and an appropriate suite of radionuclides. At the same time, the remaining WAG 1 monitoring wells and upgraded piezometers were sampled for TCL analyses. It is understood that the RCRA regulations require analyses for Appendix IX constituents only once. The subsequent list of analytes may be limited to contaminants identified through Appendix IX sampling or from knowledge of site conditions and materials that may be present. Based on these considerations, samples are currently analyzed for the TCL, an appropriate suite of radionuclides, and general water quality parameters such as total suspended solids and alkalinity.

\section{COMMENT 36:}

Page A8-1. The field logbook procedures were not included for review.

\section{RESPONSE:}

Project Procedure 1631, Logbook Protocol, is included as Attachment B, Part B - RCRA Specific Comments, Response to Specific Comment 36. 
COMMENT 37:

Page A8-2. Photograph procedures were not included for review.

\section{RESPONSE:}

Project Procedure 1110.1, Field Photography and Control, is included as Attachment B, Part B RCRA Specific Comments, Response to Specific Comment 37.

\section{COMMENT 38:}

Page A9-1. Project Procedure 1604 was not included for review.

\section{RESPONSE:}

This has been replaced by Project Procedure 1603, Sample Information Management System, which is included as Attachment B, Part B - RCRA Specific Comments, Response to Specific Comment 38.

\section{COMMENT 39:}

Page A9-2. Table A9-1--Water samples for metals analyses should be footnoted, or some other notation should be made, that mercury has a holding time of 28 days rather than 6 months.

\section{RESPONSE:}

This has been corrected.

\section{COMMENT 40:}

Page A9-3. $\mathrm{NaOH}$ is added to samples for cyanide analysis to raise the $\mathrm{pH}$ to greater than (>) 12 .

RESPONSE:

Agree.

\section{COMMENT 41:}

Page A10-1. The ORNL RI/FS ES\&H Plan was not included for review. 


\section{RESPONSE:}

This document was provided in Revision 1 of the RI Plan (August 1989).

\section{COMMENT 42:}

Page A10-4. Attachment A-3 was not included in this submittal.

\section{RESPONSE:}

All missing attachments were included in Revision 1 of the RI Plan (August 1989).

\section{COMMENT 43:}

Page A10-5. Define "...with reasonable confidence...."

\section{RESPONSE:}

If it is determined through initial sampling of a given area that mercury is not present, a decision to discontinue mercury analyses in a given area is influenced by a number of factors, including:

- Likelihood of mercury contamination based on history of spills in the area.

- Previous measurements of mercury contamination in the area.

- Potential for mercury to have been transported to the area from a known spill or leak site.

- Number of samples taken in the area and found to be free of mercury contamination.

Statistical methods are used where appropriate. 


\section{REFERENCES}

Ashwood (1988): T. L. Ashwood, Flow in the Pipeline Trenches in Main Plant Area (WAG 1): A Status Report on FY 1988 Activities, ORNL/RAP/LTR-88/54, Oak Ridge National Laboratory, 1988.

Autrey et al. (1989): J. W. Autrey et al., Sampling and Analysis of the Inactive Waste Storage Tank Contents at ORNL, ORNL/RAP-53, Oak Ridge National Laboratory, 1989.

Autrey et al. (1990a): J. W. Autrey et al., Sampling and Analysis of the Inactive Waste Storage Tank Contents at ORNL, ORNL/ER-13, Oak Ridge National Laboratory, 1990.

Autrey et al. (1990b): J. W. Autrey et al., Sampling and Analysis of the Inactive Waste Tanks TH2, WC-1, and WC-15, ORNL/ER-19, Oak Ridge National Laboratory, 1990.

BNI (1988): Bechtel National Inc., Team, Quality Assurance Plan for the Oak Ridge National Laboratory Remedial Investigation/Feasibility Study, ORNL/RAP/Sub-87/99053/13, Oak Ridge, Tennessee, 1988.

Cerling and Huff (1986): T. E. Cerling and D. D. Huff, 1985 White Oak Creek and Tributaries Streambed Contaminant Survey, ORNL/RAP/LTR-86/47, Oak Ridge National Laboratory, 1986.

Cerling and Spalding (1981): T. E. Cerling and B. P. Spalding, Areal Distribution of Co-60, Cs-137, and Sr-90 in Streambed Gravels of White Oak Creek Watershed, Oak Ridge, Tennessee, ORNL/TM-7318, Oak Ridge National Laboratory, 1981.

Cutshall (1985): N. H. Cutshall, Intralaboratory memo to D. D. Huff on Mini-Survey of Fifth Creek Sediments, Oak Ridge National Laboratory, 1985.

Daniels (1989): K. Daniels, Oak Ridge National Laboratory, personal communication to J. T. Kitchings, BNI Team, on Sediments from 7500 Bridge Area, June 1989.

EPA (1988a): U. S. Environmental Protection Agency, Superfund Exposure Assessment Manual, EPA/540/1-88/001, OSWER Directive 9285.5-1, Washington, D.C., 1988

EPA (1988b): U.S. Environmental Protection Agency, Guidance for Condusting Remedial Investigations and Feasibility Studies under CERCLA, OSWER 9355.3-01, Washington, D.C., 1988.

EPA (1989): U.S. Environmental Protection Agency, Risk Assessment Guidance for Superfund Volumes I and II, EPA/540/1-89/002, Washington, D.C., 1989.

Geo-Centers, Inc. (1982): Geo-Centers, Inc., Ground-penetrating Radar-Proof of Principle Test, ORNL Internal Report GC-TR-82-299, Oak Ridge National Laboratory, 1982. 
Grimsby (1986): H. J. Grimsby, Identification of Low-Level Waste Line Leak Sites at Oak Ridge National Laboratory, ORNL/RAP/LTR-86/8, Oak Ridge National Laboratory, 1986.

Hall (1989): D. Hall, personal communication, Volumes and Treatment Costs for Contaminated Groundwater - FY 1989, 1989.

Huang et al. (1984a): S. F. Huang, et al., Preliminary Radiological Survey of the Gunite Waste Tanks in the South Tank Farm at Oak Ridge National Laboratory, ORNL/CF-84/206, Oak Ridge National Laboratory, 1984.

Ketelle et al. (1986): R. H. Ketelle, et al., 1986 Groundwater System Characterization, ORNL/RAP/LTR-86/75, Oak Ridge National Laboratory, 1986.

Lee and Ketelle (1988): R. R. Lee and R. H. Ketelle, Subsurface Geology of the Chickamauga Group at Oak Ridge National Laboratory, ORNL/TM-10749, Oak Ridge National Laboratory, 1988.

McMaster (1963): W. M. McMaster, Geologic Map of the Oak Ridge Reservation, Tennessee, ORNL/TM-713, Oak Ridge National Laboratory, 1963.

Melroy (1986): L. A. Melroy, Wet Weather Dye Tracer Study, ORNL/RAP/86-42, Oak Ridge National Laboratory, 1986.

MMES (1986): Martin Marietta Energy Systems, Inc., Environmental Surveillance of the Oak Ridge Reservation and Surrounding Environs During 1985, ORNL/TM-6271, Oak Ridge National Laboratory, 1986.

MMES (1987): Martin Marietta Energy Systems, Inc., Environmental Surveillance of the U.S. Department of Energy Oak Ridge Reservation and Surrounding Environs During 1986, ES/ESH1/V1, Oak Ridge National Laboratory, 1987.

MMES (1988a): Martin Marietta Energy Systems, Inc., Environmental Surveillance Procedures Quality Control Program (Draft), ESH/Sub/87-21706/1, Oak Ridge National Laboratory, 1988.

MMES (1989): Martin Marietta Energy Systems, Inc., Environmental Surveillance of the Oak Ridge Reservation and Surrounding Environs During 1988 (Draft), Oak Ridge National Laboratory, 1989.

Moore (1989): G. K. Moore, Groundwater Parameters and Flow Systems near Oak Ridge National Laboratory, ORNL/TM-11368, Oak Ridge National Laboratory, 1989.

Morrison and Cerling (1987): S. J. Morrison and T. E. Cerling, Survey of Metal, Radionuclide and Organic Contamination at 20 Waste Area Groups (WAGs), ORNL Facilities, Oak Ridge, Tennessee, ORNL/RAP/Sub-87/27463/1, Oak Ridge National Laboratory, 1987.

Oakes T. W., 1987 (See MMES 1987). 
Oakes (1983): T. W. Oakes, Intralaboratory memo to J. F. Wing on Mercury in Soil and Sediment at ORNL, Mercury Contaminated Soil-3503, 3592, 4501 (SWMUs 1.1, 1.2, 1.3), Oak Ridge National Laboratory, June 9, 1983.

Oakes (1985): T. W. Oakes, Intralaboratory memo to B. J. Davis on 3019 and 3028 Area Soil Sampling Results, Oak Ridge National Laboratory, May 3, 1985.

Oakes and Shank (1977): T. W. Oakes and K. E. Shank, Subsurface Investigation of the Energy Systems Research Laboratory Site at ORNL, ORNL/TM-5695, Oak Ridge National Laboratory, 1977.

Rogers et al. (1989): Rogers et al., Oak Ridge Reservation Environmental Report for 1988, Vol. 1: Narrative, Summary, and Conclusions, ES/ESH-8/V1, Martin Marietta Energy Systems, Inc., May 1989.

Sears (1987): M. B. Sears, Preliminary Assessment of the Radiological Impact for Individual Waste Management Areas at the Oak Ridge National Laboratory: Status Report, ORNL/TM-10566, Oak Ridge National Laboratory, 1987.

Stockdale (1951): P. B. Stockdale, Geologic Conditions at the Oak Ridge National Laboratory (X-10) Area Relevant to the Disposal of Radioactive Waste, ORO-58, U.S. Atomic Energy Commission, Oak Ridge, Tennessee, 1951.

Taylor (1989): F. G. Taylor, Jr., Mercury Assessment for Water and Sediment in Oak Ridge National Laboratory Streams, ORNL/M-713, Oak Ridge National Laboratory, 1986.

Williams, Clark, and Crutcher (1987): J. K. Williams, C. Clark, Jr., and J. W. Crutcher, Radiological and RCRA Hazardous Waste Scoping Survey of the Storage Pad Southwest of Bldg. 3503, ORNL/RAP-4, Oak Ridge National Laboratory, 1987. 
Attachment A, CERCLA Specific Comment Response 9

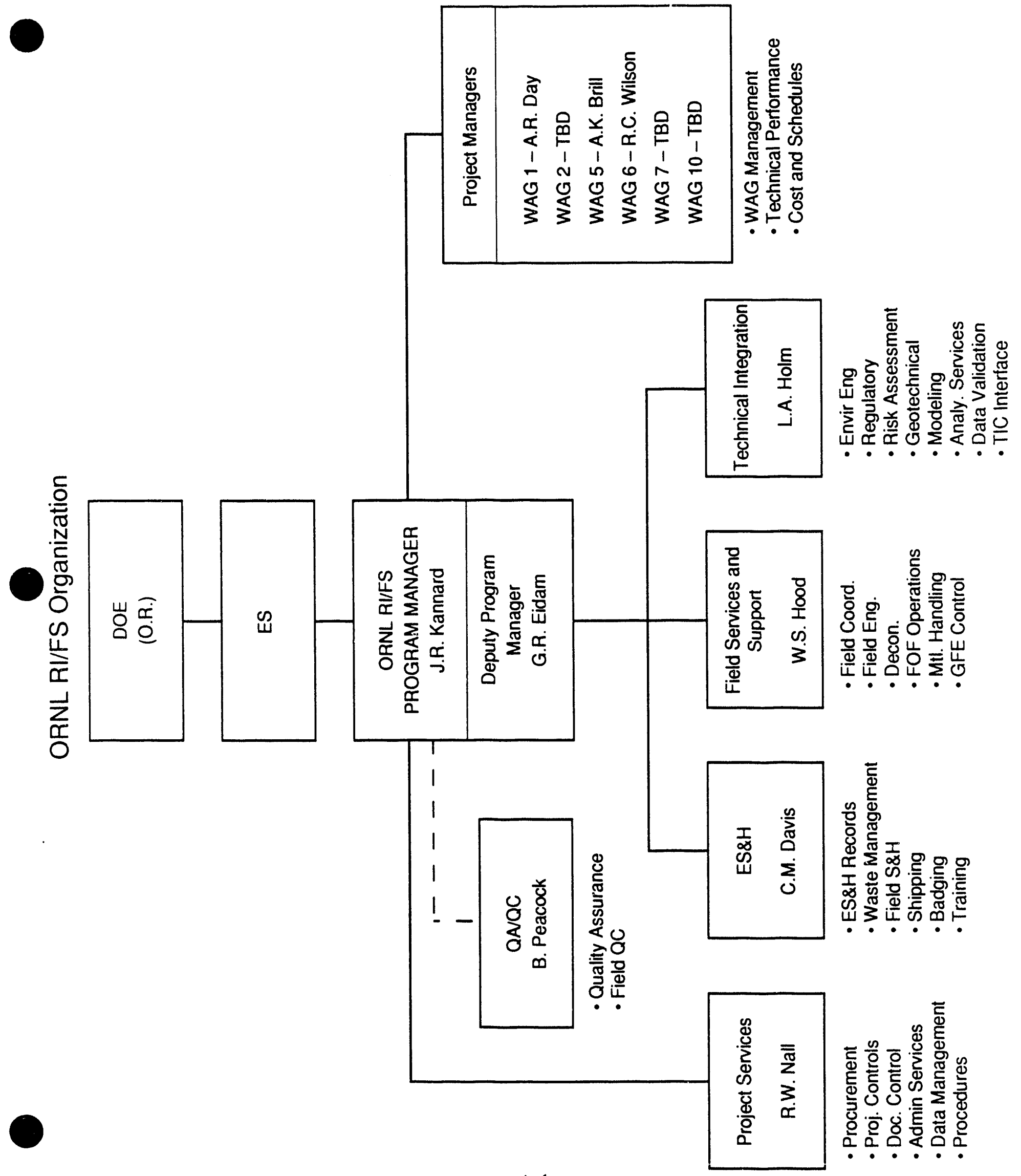




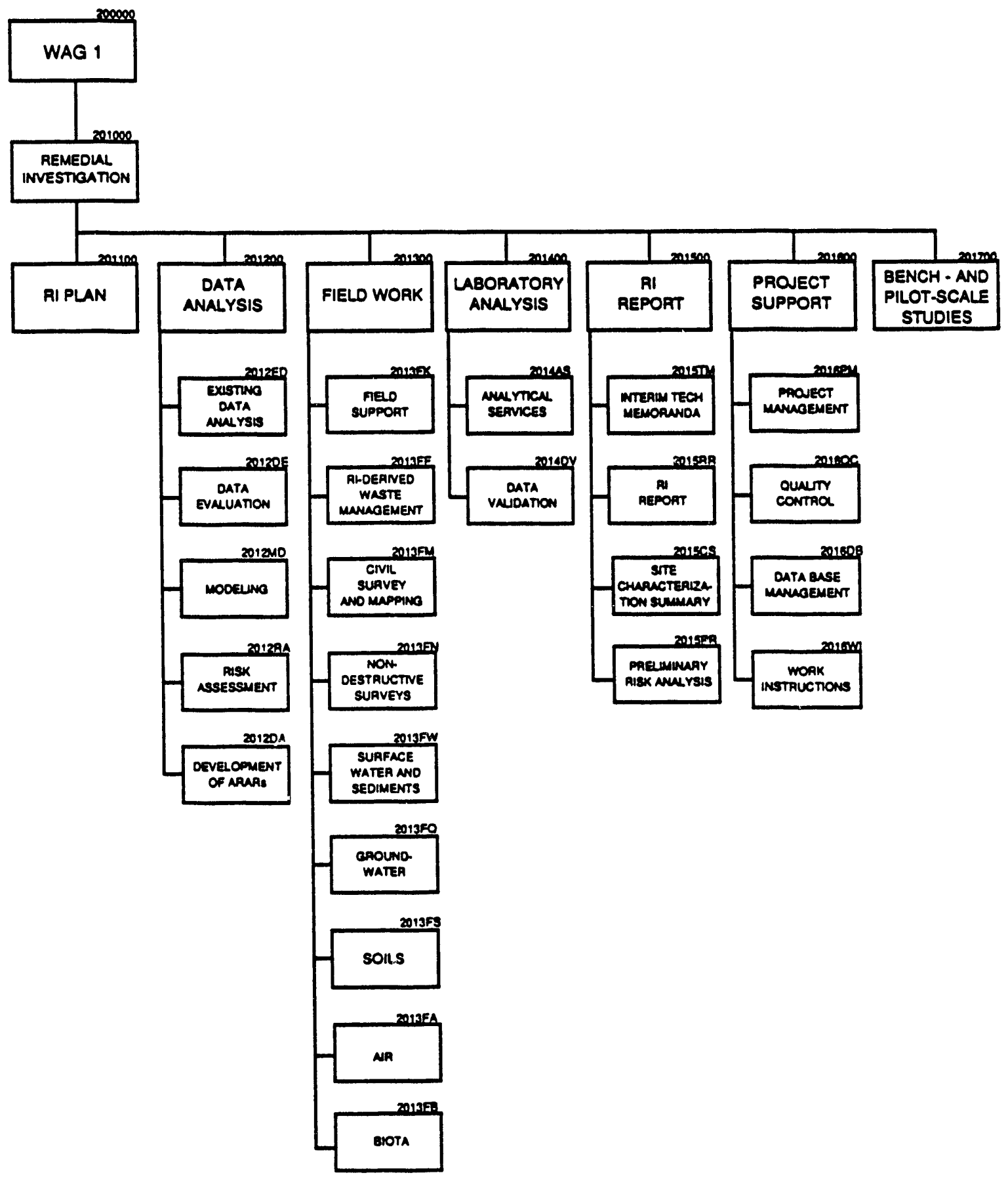

Work breakdown structure for WAG 1. 
Attachment A, CERCLA Specific Comment Response 26

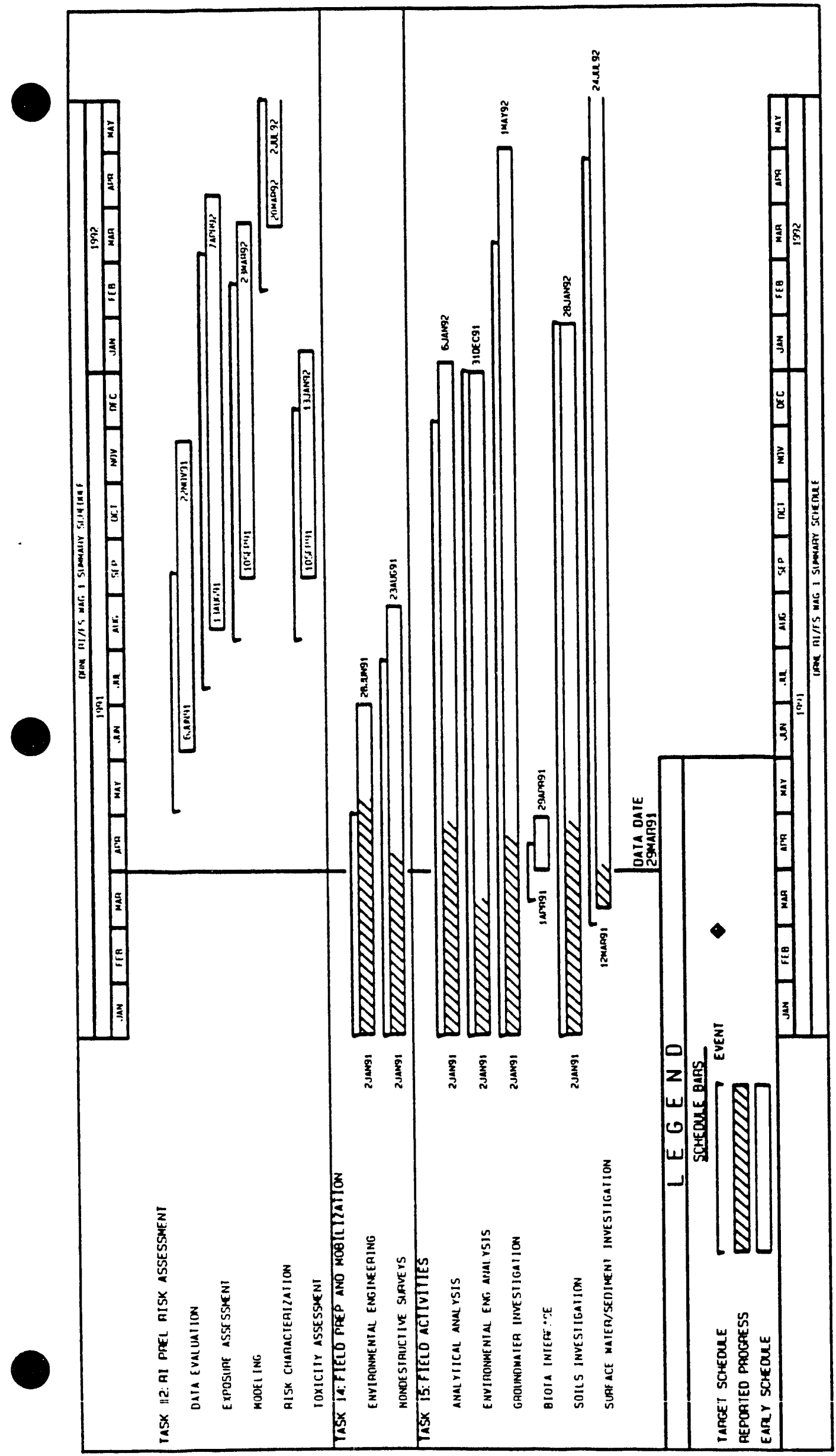


1985 concentrations of radionuclides in surface streams and tap water

\begin{tabular}{|c|c|c|c|c|c|c|}
\hline & & & ${ }^{60} \mathrm{Co}$ & ${ }^{137} \mathrm{Cs}$ & ${ }^{3} \mathrm{H}$ & ${ }^{90} \mathrm{Sr}$ \\
\hline Location ${ }^{a}$ & samples & Value & $\left(10^{-9} \mu \mathrm{Ci} / \mathrm{mL}\right)^{b}$ & $\left(10^{-9} \mu \mathrm{Ci} / \mathrm{mL}\right)^{b}$ & $\left(10^{6} \mu \mathrm{Ci} / \mathrm{mL}\right)^{\mathrm{b}}$ & $\left(10^{-9} \mu \mathrm{Ci} / \mathrm{mL}\right)^{b}$ \\
\hline $\begin{array}{l}\text { Melton Hill } \\
\text { Dam (W1) }\end{array}$ & $4^{c}$ & $\begin{array}{l}\text { Max } \\
\text { Min } \\
\text { Av }\end{array}$ & $\begin{array}{l}<0.27 \\
<0.27 \\
<0.27\end{array}$ & $\begin{array}{l}2.1 \\
0.27 \\
1.1\end{array}$ & $\begin{array}{l}5.1 \\
0.0 \\
2.5\end{array}$ & $\begin{array}{l}1.4 \\
0.081 \\
0.79\end{array}$ \\
\hline $\begin{array}{l}\text { ORGDP process } \\
\text { water (W20) }\end{array}$ & 4 & $\begin{array}{l}\text { Max } \\
\text { Min } \\
\text { Av }\end{array}$ & $\begin{array}{l}0.22 \\
0.11 \\
0.14\end{array}$ & $\begin{array}{l}0.32 \\
0.081 \\
0.17\end{array}$ & $\begin{array}{l}5.3 \\
0.38 \\
2.5\end{array}$ & $\begin{array}{l}3.2^{\mathrm{d}} \\
0.46 \\
1.9\end{array}$ \\
\hline $\begin{array}{l}\text { Kingston } \\
\text { (W45) }\end{array}$ & 4 & $\begin{array}{l}\text { Max } \\
\text { Min } \\
\text { Av }\end{array}$ & $\begin{array}{l}0.16 \\
0.054 \\
0.099\end{array}$ & $\begin{array}{l}0.081 \\
0.054 \\
0.072\end{array}$ & $\begin{array}{l}0.48 \\
0.0 \\
0.12\end{array}$ & $\begin{array}{l}3.2 \\
0.16 \\
1.2\end{array}$ \\
\hline $\begin{array}{l}\text { ORNL tap } \\
\text { water }\end{array}$ & $\vdots$ & $\begin{array}{l}\text { Max } \\
\text { Min } \\
\text { Av }\end{array}$ & $\begin{array}{l}0.11 \\
0.081 \\
0.099\end{array}$ & $\begin{array}{l}0.1 \\
0.081 \\
0.090\end{array}$ & $\begin{array}{l}0.0 \\
0.0 \\
0.0\end{array}$ & $\begin{array}{l}1.1 \\
0.027 \\
0.42\end{array}$ \\
\hline $\begin{array}{l}\text { Confluence of } \\
\text { White Oak Creek } \\
\text { and Clinch River } \\
\text { (W2) }\end{array}$ & 52 & $\begin{array}{l}\text { Max } \\
\text { Min } \\
\mathrm{Av}\end{array}$ & $\begin{array}{l}170 \\
<0.81 \\
<10\end{array}$ & $\begin{array}{l}1500 \\
<1.6 \\
<46\end{array}$ & $\begin{array}{c}350 \\
5.4 \\
62\end{array}$ & $\begin{array}{c}350 \\
1.4 \\
52\end{array}$ \\
\hline $\begin{array}{l}\text { White Oak } \\
\text { Dam (W3) }\end{array}$ & 52 & $\begin{array}{l}\text { Max } \\
\text { Min } \\
\text { Av }\end{array}$ & $\begin{array}{c}1900 \\
5.0 \\
63\end{array}$ & $\begin{array}{c}650 \\
6.9 \\
42\end{array}$ & $\begin{array}{r}780 \\
83 \\
350\end{array}$ & $\begin{array}{r}3500 \\
110 \\
300\end{array}$ \\
\hline
\end{tabular}

asee Fig. 3.5.

${ }^{\mathrm{b}} \mu \mathrm{Ci}=10^{-6} \mathrm{Ci}=3.7 \times 10^{-4} \mathrm{~d} / \mathrm{s}$.

'Except for ${ }^{3} \mathrm{H}$-only 2 samples.

${ }^{d}$ Sample had a high (about 100\%) counting uncertainty which also affected the average value.

Source: Martin Marietta Energy Systems, Inc., Environmental Surveillance of the Oak Ridge Reservation and Surrounding Environs During 1985, ORNL/TM-6271, Oak Ridge National Laboratory,
1986. 
Attachment A, CERCLA Specific Comment

Response 37

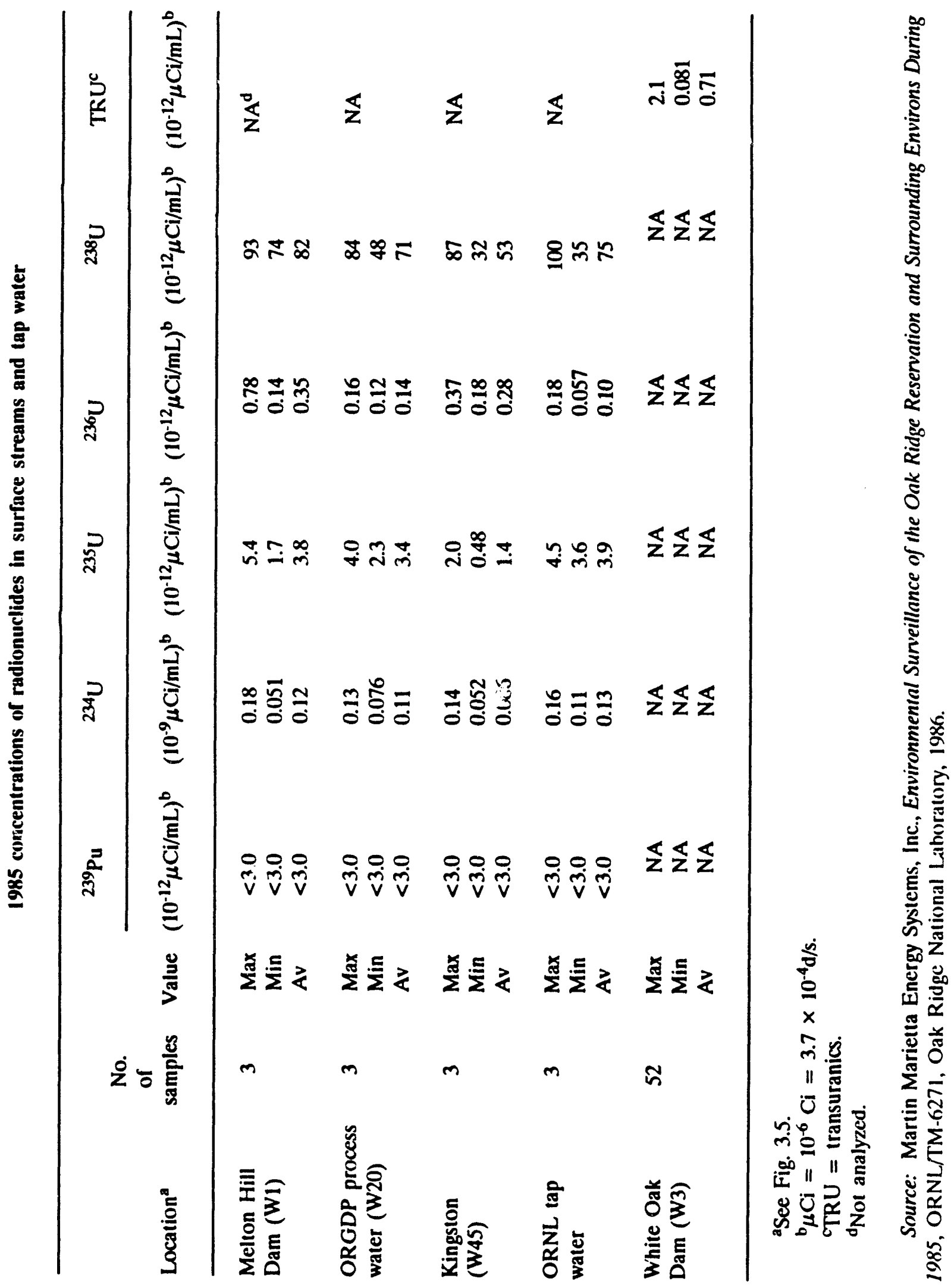


Attachment A, CERCLA Specific Comment Response 45

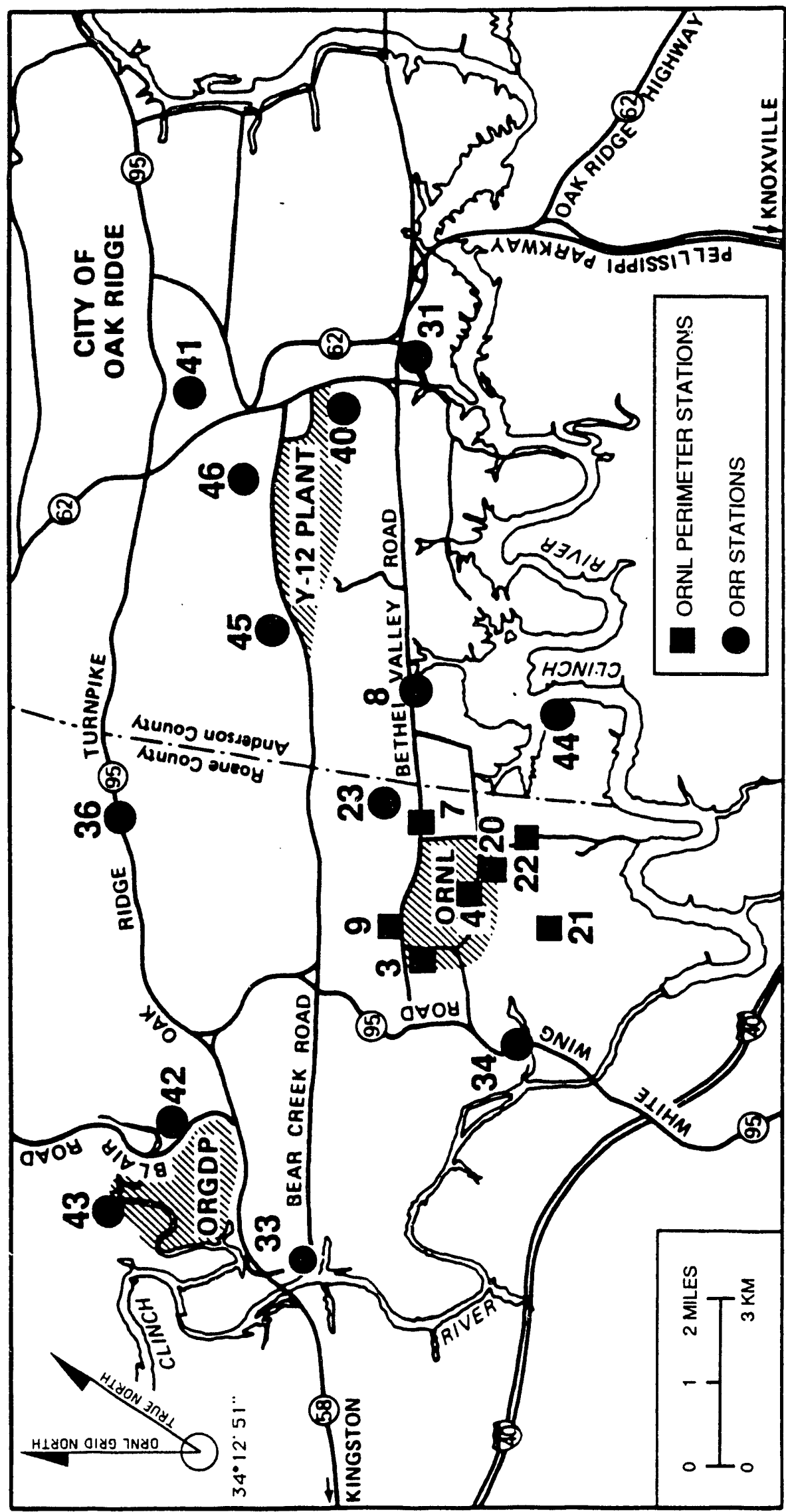

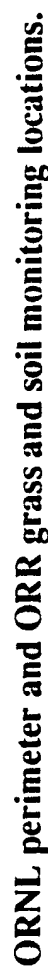


Attachment A, CERCLA Specific Comment Response 45

1988 concentrations of radionuclides in grass

\begin{tabular}{lllllll}
\hline \multirow{2}{*}{ Location } & & \multicolumn{4}{c}{ Concentration (pCi/g ash wt) } \\
\cline { 5 - 7 } & Radionuclides & $\begin{array}{l}\text { Number of } \\
\text { samples }\end{array}$ & Max & Min & Av & $\begin{array}{l}\text { Standard } \\
\text { error }^{\mathrm{c}}\end{array}$ \\
\hline ORNL & ${ }^{60} \mathrm{Co}$ & 28 & 0.11 & -0.31 & 0.012 & 0.0057 \\
perimeter & ${ }^{137} \mathrm{Cs}$ & 28 & 2.2 & -0.090 & 0.28 & 0.13 \\
stations & ${ }^{238} \mathrm{Pu}$ & 28 & 0.0030 & -0.0030 & -0.00010 & 0.00025 \\
& ${ }^{239} \mathrm{Pu}$ & 28 & 0.022 & -0.0043 & 0.0010 & 0.0010 \\
& Total Sr & 28 & 22 & 0.019 & 2.2 & 1.0 \\
& ${ }^{234} \mathrm{U}$ & 28 & 0.10 & 0.0073 & 0.030 & 0.0044 \\
& ${ }^{235} \mathrm{U}$ & 28 & 0.011 & -0.0015 & 0.0028 & 0.00056 \\
& ${ }^{238} \mathrm{U}$ & 28 & 0.089 & 0.0027 & 0.019 & 0.0035 \\
Oak Ridge & ${ }^{60} \mathrm{Co}$ & 52 & 0.087 & -0.074 & 0.011 & 0.0041 \\
Reservation & ${ }^{137} \mathrm{Cs}$ & 52 & 0.11 & -0.040 & 0.015 & 0.0044 \\
stations & ${ }^{238} \mathrm{Pu}$ & 52 & 0.0035 & -0.0024 & 0.00024 & 0.00017 \\
& ${ }^{239} \mathrm{Pu}$ & 52 & 0.0022 & -0.010 & -0.0018 & 0.00032 \\
& ${ }^{23}$ & 0.51 & -0.11 & 0.16 & 0.019 \\
& ${ }^{234} \mathrm{U}$ & 52 & 0.15 & 0.0068 & 0.033 & 0.0045 \\
& ${ }^{235} \mathrm{U}$ & 52 & 0.013 & -0.00054 & 0.0031 & 0.00043 \\
& ${ }^{238} \mathrm{U}$ & 52 & 0.14 & 0.002 & 0.019 & 0.0043 \\
\hline
\end{tabular}

asee map attachment to this response.

bSome radionuclides are reported without regard to lower limits of detection. This practice, approved by DOE and EPA, can result in values below zero (after correcting for background).

'Standard deviation about the average.

${ }^{\mathrm{d}}$ Total radioactive strontium $\left({ }^{89} \mathrm{Sr}+{ }^{90} \mathrm{Sr}\right)$. 
Attachment A, CERCLA Specific Comment Response 45

1988 radionuclide concentrations from grasses sampled at ORNL perimeter stations

\begin{tabular}{|c|c|c|c|c|c|}
\hline \multirow[b]{2}{*}{ Location $^{\mathbf{a}}$} & \multirow[b]{2}{*}{$\begin{array}{l}\text { Number of } \\
\text { samples }\end{array}$} & \multicolumn{4}{|c|}{$\begin{array}{l}\text { Concentration } \\
\text { (pCi/g ash wi) }\end{array}$} \\
\hline & & $\operatorname{Max}$ & Min & Av & $\begin{array}{l}\text { Standard } \\
\text { error }^{b}\end{array}$ \\
\hline \multicolumn{6}{|l|}{${ }^{60} \mathrm{Co}$} \\
\hline 3 & 4 & 0.0073 & -0.027 & -0.0092 & 0.0083 \\
\hline 4 & 4 & 0.063 & 0.019 & 0.040 & 0.011 \\
\hline 7 & 4 & 0.041 & -0.031 & -0.0044 & 0.016 \\
\hline 9 & 4 & 0.11 & 0.0090 & 0.042 & 0.022 \\
\hline 20 & 4 & 0.021 & -0.021 & -0.00044 & 0.010 \\
\hline 21 & 4 & 0.025 & 0.0021 & 0.011 & 0.0050 \\
\hline 22 & 4 & 0.030 & -0.016 & 0.0063 & 0.011 \\
\hline $\begin{array}{l}\text { Network } \\
\text { summary }\end{array}$ & 28 & 0.11 & -0.031 & 0.012 & 0.0057 \\
\hline \multicolumn{6}{|l|}{${ }^{137} \mathrm{Cs}$} \\
\hline 3 & 4 & 0.064 & 0.022 & 0.044 & 0.0093 \\
\hline 4 & 4 & 2.2 & 1.2 & 1.8 & 0.24 \\
\hline 7 & 4 & 0.069 & -0.0017 & 0.037 & 0.015 \\
\hline 9 & 4 & 0.077 & -0.090 & 0.021 & 0.038 \\
\hline 20 & 4 & 0.043 & -0.0036 & 0.013 & 0.010 \\
\hline 21 & 4 & 0.013 & -0.012 & 0.0024 & 0.0056 \\
\hline 22 & 4 & 0.046 & -0.0055 & 0.011 & 0.012 \\
\hline $\begin{array}{l}\text { Network } \\
\text { summary }\end{array}$ & 28 & 2.2 & -0.090 & 0.28 & 0.13 \\
\hline \multicolumn{6}{|l|}{${ }^{238} \mathrm{Pu}$} \\
\hline 3 & 4 & 0.0011 & -0.0030 & -0.0011 & 0.00087 \\
\hline 4 & 4 & 0.0030 & 0.00027 & 0.0018 & 0.00059 \\
\hline 7 & 4 & -0.000076 & -0.0019 & -0.00061 & 0.00043 \\
\hline 9 & 4 & 0.0012 & -0.0011 & -0.00020 & 0.00053 \\
\hline 20 & 4 & 0.0014 & -0.0010 & -0.00016 & 0.00055 \\
\hline 21 & 4 & 0.00043 & -0.00015 & 0.000099 & 0.00013 \\
\hline 22 & 4 & 0.00081 & -0.0018 & -0.00058 & 0.00054 \\
\hline $\begin{array}{l}\text { Network } \\
\text { summary }\end{array}$ & 28 & 0.0030 & -0.0030 & -0.00010 & 0.00025 \\
\hline
\end{tabular}


Attachment A, CERCLA Specific Comment Response 45

\begin{tabular}{|c|c|c|c|c|c|}
\hline \multirow[b]{2}{*}{ Location $^{\mathrm{a}}$} & \multirow[b]{2}{*}{$\begin{array}{l}\text { Number of } \\
\text { samples }\end{array}$} & \multicolumn{4}{|c|}{$\begin{array}{l}\text { Concentration } \\
\text { (pCi/g ash wt) }\end{array}$} \\
\hline & & $\operatorname{Max}$ & Min & Av & $\begin{array}{c}\text { Standard } \\
\text { error }\end{array}$ \\
\hline \multicolumn{6}{|l|}{${ }^{239} \mathrm{Pu}$} \\
\hline 3 & 4 & 0.00027 & -0.00089 & -0.00042 & 0.00025 \\
\hline 4 & 4 & 0.022 & 0.0016 & 0.011 & 0.0043 \\
\hline 7 & 4 & 0.00062 & -0.0043 & -0.0017 & 0.0011 \\
\hline 9 & 4 & 0.0054 & -0.0021 & 0.00019 & 0.0018 \\
\hline 20 & 4 & 0.00024 & -0.00078 & -0.00018 & 0.00022 \\
\hline 21 & 4 & -0.00014 & -0.0026 & -0.0014 & 0.00068 \\
\hline 22 & 4 & 0.0025 & -0.0021 & -0.00037 & 0.00098 \\
\hline $\begin{array}{l}\text { Network } \\
\text { summary }\end{array}$ & 28 & 0.022 & -0.0043 & 0.0010 & 0.0010 \\
\hline \multicolumn{6}{|l|}{ Total $\mathrm{Sr}^{\mathrm{C}}$} \\
\hline 3 & 4 & 0.32 & 0.089 & 0.17 & 0.053 \\
\hline 4 & 4 & 22 & 8.9 & 14 & 3.2 \\
\hline 7 & 4 & 0.22 & 0.092 & 0.13 & 0.031 \\
\hline 9 & 4 & 0.35 & 0.019 & 0.22 & 0.076 \\
\hline 20 & 4 & 0.12 & 0.046 & 0.084 & 0.016 \\
\hline 21 & 4 & 0.25 & 0.14 & 0.20 & 0.024 \\
\hline 22 & 4 & 0.23 & 0.095 & 0.15 & 0.029 \\
\hline $\begin{array}{l}\text { Network } \\
\text { summary }\end{array}$ & 28 & 22 & 0.019 & 2.2 & 1.0 \\
\hline \multicolumn{6}{|l|}{${ }^{234} \mathrm{U}$} \\
\hline 3 & 4 & 0.059 & 0.010 & 0.024 & 0.012 \\
\hline 4 & 4 & 0.051 & 0.019 & 0.037 & 0.0067 \\
\hline 7 & 4 & 0.10 & 0.025 & 0.055 & 0.017 \\
\hline 9 & 4 & 0.030 & 0.0073 & 0.016 & 0.0050 \\
\hline 20 & 4 & 0.068 & 0.030 & 0.050 & 0.0095 \\
\hline 21 & 4 & 0.035 & 0.0097 & 0.020 & 0.0058 \\
\hline 22 & 4 & 0.015 & 0.0078 & 0.011 & 0.0015 \\
\hline $\begin{array}{l}\text { Network } \\
\text { summary }\end{array}$ & 28 & 0.10 & 0.0073 & 0.030 & 0.0044 \\
\hline
\end{tabular}


Attachment A, CERCLA Specific Comment Response 45

\begin{tabular}{cccccc}
\hline & & \multicolumn{5}{c}{$\begin{array}{c}\text { Concentration } \\
\text { (pCi/g ash wt) }\end{array}$} \\
\cline { 3 - 6 } Location $^{\mathrm{a}}$ & $\begin{array}{c}\text { Number of } \\
\text { samples }\end{array}$ & Max & Min & $\begin{array}{c}\text { Standard } \\
\text { error }^{\mathrm{b}}\end{array}$ \\
\hline${ }^{235} \mathrm{U}$ & & & & \\
3 & 4 & 0.011 & 0.00041 & 0.0034 & 0.0025 \\
4 & 4 & 0.0049 & -0.00011 & 0.0024 & 0.0012 \\
7 & 4 & 0.0070 & 0.0041 & 0.0057 & 0.00067 \\
9 & 4 & 0.0022 & -0.00027 & 0.0010 & 0.00051 \\
20 & 4 & 0.0070 & 0.0035 & 0.0057 & 0.00085 \\
21 & 4 & 0.0030 & 0.00070 & 0.0014 & 0.00052 \\
22 & 4 & 0.0012 & -0.0015 & 0.000041 & 0.00056 \\
Network & & & & & \\
summary & 28 & 0.011 & -0.0015 & 0.0028 & 0.00056 \\
$238 \mathrm{U}$ & & & & & \\
3 & & & & & \\
4 & 4 & 0.032 & 0.0051 & 0.013 & 0.0064 \\
7 & 4 & 0.041 & 0.013 & 0.021 & 0.0066 \\
9 & 4 & 0.089 & 0.010 & 0.041 & 0.017 \\
20 & 4 & 0.025 & 0.0041 & 0.014 & 0.0045 \\
21 & 4 & 0.046 & 0.013 & 0.029 & 0.0091 \\
22 & 4 & 0.020 & 0.0054 & 0.011 & 0.0033 \\
Network & 4 & 0.0076 & 0.0027 & 0.0051 & 0.0010 \\
summary & 28 & 0.089 & 0.0027 & 0.019 & 0.0035 \\
\hline
\end{tabular}

${ }^{a}$ Location map included in attachments to this response.

${ }^{b}$ Standard deviation about the average.

cTotal radioactive strontium $\left({ }^{89} \mathrm{Sr}+{ }^{90} \mathrm{Sr}\right)$. 
Inventories of major contaminants at the Main Plant Area (WAG 1) SWMUs

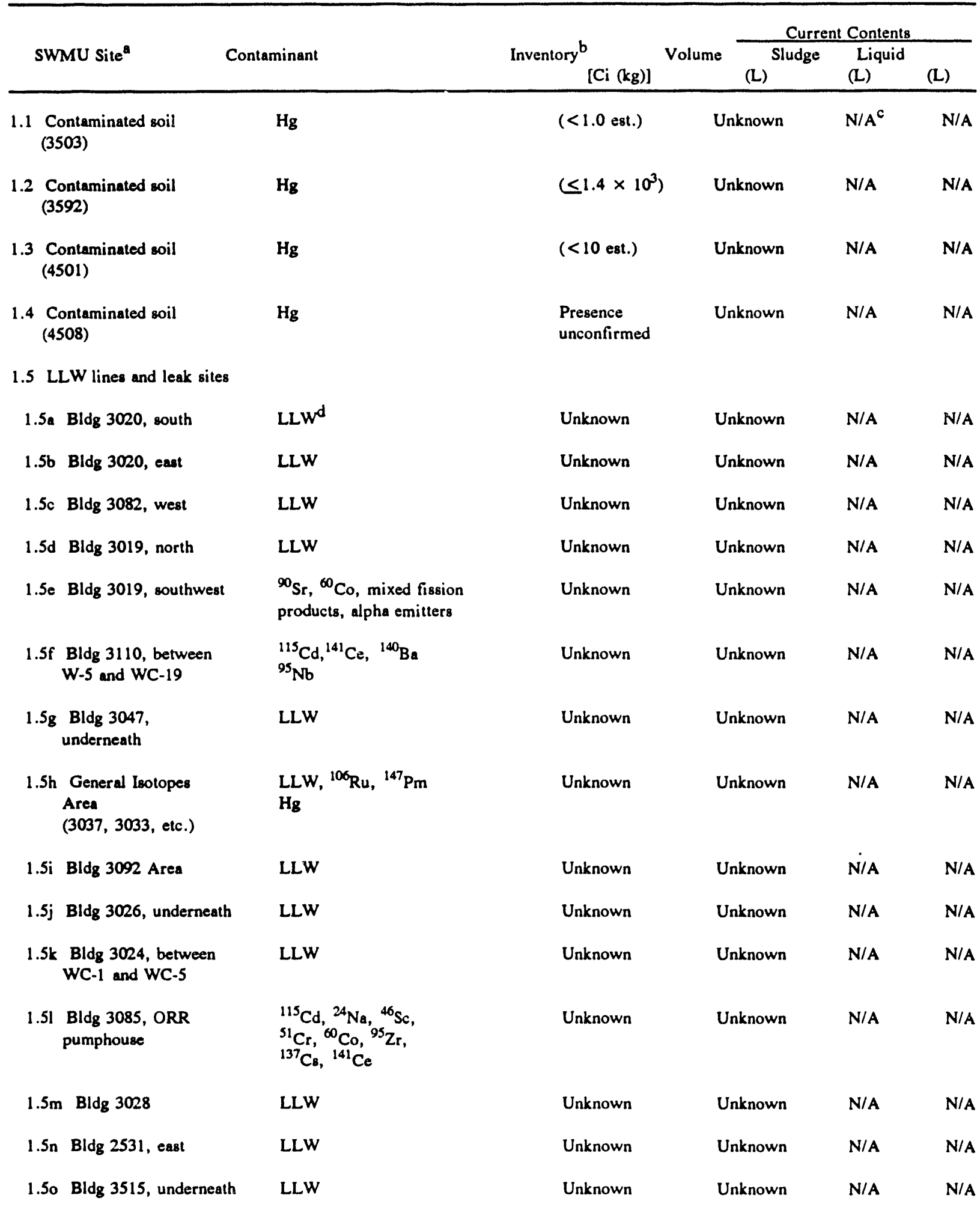




\begin{tabular}{|c|c|c|c|c|c|c|}
\hline & & & & Cur & rent Conten & \\
\hline & WMU Site ${ }^{a}$ & Contaminant & $\begin{array}{l}\text { Inventory } \\
{[\mathrm{Ci}(\mathrm{kg})]}\end{array}$ & $\begin{array}{l}\text { Volume } \\
\text { (L) }\end{array}$ & $\begin{array}{l}\text { Sludge } \\
\text { (L) }\end{array}$ & $\begin{array}{l}\text { Liquid } \\
\text { (L) }\end{array}$ \\
\hline $1.5 p$ & p Bldg 3525 , to a sump & LLW & Unknown & Unknown & N/A & N/A \\
\hline $1.5 q$ & q Bldg 3550, underneath & LLW & Unknown & Unknown & N/A & N/A \\
\hline $1.5 \mathrm{r}$ & ir Bldg 3500, sewer & LLW & Unknown & Unknown & N/A & $\mathrm{N} / \mathrm{A}$ \\
\hline $1.5 \mathrm{~s}$ & $\begin{array}{l}\text { is Abandoned line, Central } \\
\text { Avenue }\end{array}$ & LLW & Unknown & Unknown & N/A & N/A \\
\hline $1.5 \mathrm{t}$ & it Bldg 4508, north & LLW & Unknown & Unknown & N/A & N/A \\
\hline $1.5 \mathrm{u}$ & iu Bldg 3518, west & ${ }^{90} \mathrm{Sr},{ }^{137} \mathrm{Cs}$ & Unknown & Unknown & N/A & N/A \\
\hline $1.5 v$ & iv Northwest of SWSA 1 & LLW & Unknown & Unknown & N/A & N/A \\
\hline $1.5 \mathrm{w}$ & $\begin{array}{l}\text { iw Bldg 3503, ground } \\
\text { contamination }\end{array}$ & LLW & Unknown & Unknown & N/A & N/A \\
\hline $\begin{aligned} 1.6 \mathrm{C} \\
\mathrm{a} \\
\text { in }\end{aligned}$ & $\begin{array}{l}\text { Contaminated surfaces } \\
\text { and soil from } 1959 \text { explosion } \\
\text { in Bldg. } 3019 \text { cell }\end{array}$ & ${ }^{239} \mathrm{Pu}$ & $<4.7 \times 10^{2}$ & Unknown & N/A & N/A \\
\hline $1.7 \mathrm{C}$ & $\begin{array}{l}\text { Contamination at base of } \\
3019 \text { stack }\end{array}$ & $\begin{array}{l}{ }^{60} \mathrm{Co},{ }^{137} \mathrm{Cs},{ }^{244} \mathrm{Cm} \\
{ }^{241} \mathrm{Am},{ }^{228} \mathrm{Pu},{ }^{239} \mathrm{Pu}\end{array}$ & $\begin{array}{l}\text { Presence } \\
\text { unconfirmed }\end{array}$ & Unknown & N/A & $N / A$ \\
\hline $1.8 \mathrm{c}$ & $\begin{array}{l}\text { Graphite reactor storage } \\
\text { canal overflow }(3001 / 3019)\end{array}$ & Unidentified & $\begin{array}{l}\text { Presence } \\
\text { unconfirmed }\end{array}$ & Unknown & Unknown & $N / A$ \\
\hline $1.9 \mathrm{C}$ & $\begin{array}{l}\text { ORRR decay tank } \\
\text { rupture site (3087) }\end{array}$ & $\begin{array}{l}\text { Neutron activation } \\
\text { products }\end{array}$ & $\begin{array}{l}\text { Presence } \\
\text { unconfirmed }\end{array}$ & Unknown & Unknown & N/A \\
\hline $1.10 \mathrm{~s}$ & Storage pads $(3503,3504)$ & ${ }^{233} \mathrm{U},{ }^{239} \mathrm{Pu}$ & $<1.0 \times 10^{-1}$ est. & Unknown & Unknown & Unknown \\
\hline $1.11 \mathrm{I}$ & $\begin{array}{l}\text { Decommissioned waste } \\
\text { holding basin ( } 3512 \text { ) }\end{array}$ & Unidentified & $<1.0 \times 10^{1}$ est. & $113,550^{e}$ & Unknown & Unknown \\
\hline $1.12 \mathrm{~V}$ & Waste holding basin (3513) & $\begin{array}{l}\mathrm{PCBs} \\
{ }_{90}^{90} \mathrm{Sr} \\
{ }^{137} \mathrm{Cs} \\
{ }^{239} \mathrm{Pu}\end{array}$ & $\begin{array}{l}\text { Unknown } \\
3.0 \times 10^{1} \\
2.0 \times 10^{2} \\
5.0\end{array}$ & $7,115,800^{f}$ & 832,700 & $6,056,000$ \\
\hline $1.13 \mathrm{E}$ & Equalization basin (3524) & $\begin{array}{l}{ }^{90} \mathrm{Sr} \\
{ }^{137} \mathrm{Cs} \\
\text { Th, U, TRU }\end{array}$ & $\begin{array}{l}3.0 \times 10^{1} \\
1.0 \times 10^{2} \\
1.1 \times 10^{1}\end{array}$ & $3,785,000$ & Unknown & Variable \\
\hline $1.14 \mathrm{P}$ & Process waste pond (3539) & Unidentified & $<1.0 \times 10^{1}$ est. & 567,750 & Unknown & Variable \\
\hline $1.15 \mathrm{P}$ & Process waste pond (3540) & Unidentified & $<1.0 \times 10^{1}$ est. & 567,750 & Unknown & Variable \\
\hline $\begin{array}{r}1.16 \mathrm{E} \\
\mathrm{p}\end{array}$ & $\begin{array}{l}\text { East sewage aeration } \\
\text { pond }(2543)\end{array}$ & Unidentified & $<1.0 \times 10^{1}$ est. & $3,785,000$ & Unknown & Unknown \\
\hline $1.17 \mathrm{p}$ & $\begin{array}{l}\text { West sewage aeration } \\
\text { pond }(2544)\end{array}$ & Unidentified & $<1.0 \times 10^{1}$ est. & $3,785,000$ & Unknown & Variable \\
\hline
\end{tabular}


Attachment A, CERCLA Specific Comment Response 49

\begin{tabular}{|c|c|c|c|c|c|}
\hline \multirow[b]{2}{*}{ SWMU Site ${ }^{a}$} & \multirow[b]{2}{*}{ Contaminant } & \multirow[b]{2}{*}{$\begin{array}{l}\text { Inventory } \\
{[\mathrm{Ci}(\mathrm{kg})]}\end{array}$} & \multicolumn{3}{|c|}{ Current Contents } \\
\hline & & & $\begin{array}{l}\text { Volume } \\
\text { (L) }\end{array}$ & $\begin{array}{l}\text { Sludge } \\
\text { (L) }\end{array}$ & $\begin{array}{l}\text { Liquid } \\
\text { (L) }\end{array}$ \\
\hline 1.19 LITR pond (3085W) & $\begin{array}{l}{ }^{137} \mathrm{Cs} \\
{ }^{90} \mathrm{Sr} \\
{ }^{239} \mathrm{Pu}\end{array}$ & $\begin{array}{c}20 \times 10^{-3} \\
1 \times 10^{-3} \\
0.1 \times 10^{-3}\end{array}$ & $68,130^{e}$ & Unknown & N/A \\
\hline 1.20 Filter pit (3517) & ${ }^{90} \mathrm{Sr},{ }^{137} \mathrm{Cs}$ & Unknown & Unknown & Unknown & Unknown \\
\hline 1.21 FPDL LLW transfer line & Unidentified & $<1.0 \times 10^{3}$ est. & Unknown & Unknown & Unknown \\
\hline $\begin{array}{l}1.22 \text { Isotopes ductwork } \\
\text { filter house ( } 3110)\end{array}$ & Unidentified & Unknown & Unknown & Unknown & Unknown \\
\hline 1.23a Inactive tanks (W-1 $)^{g}$ & $\begin{array}{l}{ }^{90} \mathrm{Sr} \\
{ }^{137} \mathrm{Cs} \\
{ }^{152} \mathrm{Eu} \\
{ }^{154} \mathrm{Eu} \\
\text { TRU }\end{array}$ & $\begin{array}{l}9.0 \times 10^{-2} \\
2.0 \times 10^{-2} \\
3.0 \times 10^{-2} \\
5.0 \times 10^{-4} \\
7.0 \times 10^{-4}\end{array}$ & 18,168 & Unknown & 3,785 \\
\hline 1.23b Inactive tank (W-2) & $\begin{array}{l}{ }^{90} \mathrm{Sr} \\
{ }^{60} \mathrm{Co} \\
{ }^{137} \mathrm{Cs} \\
{ }^{152} \mathrm{Eu} \\
{ }^{154} \mathrm{Eu} \\
{ }^{155_{\mathrm{EU}}} \\
{ }^{233} \mathrm{U} \\
{ }^{235} \mathrm{U} \\
\text { TRU }\end{array}$ & $\begin{array}{l}1.0 \times 10^{1} \\
4.0 \times 10^{-2} \\
1.0 \times 10^{1} \\
5.0 \\
8.0 \times 10^{-1} \\
3.0 \times 10^{-2} \\
8.0 \times 10^{-3} \\
3.0 \times 10^{-4} \\
7.0\end{array}$ & 18,168 & 1,893 & 3,028 \\
\hline \multirow[t]{2}{*}{ 1.24a Inactive tank (W-3) } & $\begin{array}{l}{ }^{90} \mathrm{Sr} \\
{ }^{137} \mathrm{Cs} \\
{ }^{233} \mathrm{U} \\
{ }^{238} \mathrm{U}\end{array}$ & $\begin{array}{l}3.0 \times 10^{1} \\
1.0 \times 10^{3} \\
1.0 \times 10^{-2} \\
2.0 \times 10^{-3}\end{array}$ & 160,863 & 15,897 & 84,027 \\
\hline & TRU & $2.0 \times 10^{2}$ & & & \\
\hline $1.24 b$ Inactive tank $(W-4)$ & $\begin{array}{l}{ }^{90} \mathrm{Sr} \\
{ }^{137} \mathrm{Cs} \\
{ }^{233} \mathrm{U} \\
{ }^{235} \mathrm{U} \\
{ }^{228} \mathrm{U} \\
\mathrm{TRU}\end{array}$ & $\begin{array}{l}1.0 \times 10^{2} \\
1.0 \times 10^{2} \\
2.0 \\
8.0 \times 10^{-2} \\
2.0 \\
4.2\end{array}$ & 160,863 & 21,953 & 43,960 \\
\hline 1.25a Inactive tank (W-13) & $\begin{array}{l}{ }^{90} \mathrm{Sr} \\
{ }^{60} \mathrm{Co} \\
{ }^{137} \mathrm{Cs} \\
{ }^{154} \mathrm{Eu} \\
{ }^{203} \mathrm{U} \\
{ }^{235} \mathrm{U} \\
{ }^{238} \mathrm{U} \\
\text { TRU }\end{array}$ & $\begin{array}{l}3.0 \times 10^{2} \\
1.0 \times 10^{-2} \\
3.0 \times 10^{1} \\
1.0 \times 10^{-1} \\
2.0 \times 10^{-4} \\
2.0 \times 10^{-5} \\
2.0 \times 10^{-1} \\
4.3 \times 10^{-2}\end{array}$ & 7,570 & Unknown & 1,703 \\
\hline
\end{tabular}




\begin{tabular}{|c|c|c|c|c|c|}
\hline \multirow[b]{2}{*}{ SWMU Site ${ }^{a}$} & \multirow[b]{2}{*}{ Contaminant } & \multirow[b]{2}{*}{$\begin{array}{l}\text { Inventory }{ }^{b} \\
{[\mathrm{Ci}(\mathrm{kg})]}\end{array}$} & \multicolumn{3}{|c|}{ Current Contents } \\
\hline & & & $\begin{array}{c}\begin{array}{c}\text { Volume } \\
\text { (L) }\end{array} \\
\end{array}$ & $\begin{array}{c}\text { Sludge } \\
\text { (L) }\end{array}$ & $\begin{array}{c}\text { Liquid } \\
(\mathrm{L})\end{array}$ \\
\hline $1.25 \mathrm{~b}$ Inactive tank (W-14) & $\begin{array}{l}{ }^{90} \mathrm{Sr} \\
{ }^{60} \mathrm{Co} \\
{ }^{137} \mathrm{Cs} \\
{ }^{233} \mathrm{U} \\
{ }^{238} \mathrm{U} \\
\text { TRU }\end{array}$ & $\begin{array}{l}8.0 \\
3.0 \times 10^{-2} \\
6.0 \\
2.0 \times 10^{-4} \\
3.0 \times 10^{-5} \\
6.0 \times 10^{-4}\end{array}$ & 7,570 & Unknown & 4,542 \\
\hline $1.25 \mathrm{c}$ Inactive tank (W-15) & Unidentified & Unknown & 7,570 & Unknown & Unknown \\
\hline 1.26a Inactive tank (W-5) & $\begin{array}{l}{ }^{90} \mathrm{Sr} \\
{ }_{137} \mathrm{Cs} \\
{ }^{232} \mathrm{Th}, \mathrm{U}, \mathrm{TRU}\end{array}$ & $\begin{array}{r}300 \\
20 \\
10\end{array}$ & 643,450 & 22,710 & Unknown \\
\hline 1.26b Inactive tank (W-6) & $\begin{array}{l}{ }^{90} \mathrm{Sr} \\
{ }^{137} \mathrm{Cs} \\
{ }^{232} \mathrm{Th}, \mathrm{U}, \mathrm{TRU}\end{array}$ & $\begin{array}{r}2,000 \\
150 \\
40\end{array}$ & 643,450 & 56,775 & Unknown \\
\hline 1.26c Inactive tank (W-7) & $\begin{array}{l}{ }^{90} \mathrm{Sr} \\
{ }^{137} \mathrm{Cs} \\
\mathrm{Th}^{232}, \mathrm{U}, \mathrm{TRU}\end{array}$ & $\begin{array}{r}2,000 \\
150 \\
40\end{array}$ & 643,450 & Minimal & Unknown \\
\hline $1.26 \mathrm{~d}$ Inactive tank (W-8) & $\begin{array}{l}{ }^{90} \mathrm{Sr} \\
{ }^{137} \mathrm{Cs} \\
{ }^{232} \mathrm{Th}, \mathrm{U}, \mathrm{TRU}\end{array}$ & $\begin{array}{r}2,000 \\
150 \\
40\end{array}$ & 643,450 & 3,785 & Unknown \\
\hline 1.26e Inactive tank (W-9) & $\begin{array}{l}{ }^{90} \mathrm{Sr} \\
{ }^{137} \mathrm{Cs} \\
{ }^{232} \mathrm{Th}, \mathrm{U}, \mathrm{TRU}\end{array}$ & $\begin{array}{r}2,000 \\
150 \\
40\end{array}$ & 643,450 & 11,355 & Unknown \\
\hline 1.26f Inactive tank (W-10) & $\begin{array}{l}{ }^{90} \mathrm{Sr} \\
{ }^{137} \mathrm{Cs} \\
{ }^{232} \mathrm{Th}, \mathrm{U}, \mathrm{TRU}\end{array}$ & $\begin{array}{r}2,000 \\
960 \\
330\end{array}$ & 643,450 & 151,400 & Unknown \\
\hline 1.27 Inactive $\operatorname{tank}(W-11)$ & ${ }^{90} \mathrm{Sr}$ & $\begin{array}{l}1.0 \times 10^{-3} \\
1.0 \times 10^{-3}\end{array}$ & 5,678 & 170 & 984 \\
\hline 1.28 Inactive tank (W-1A) & $\begin{array}{l}{ }^{90} \mathrm{Sr}_{\mathrm{r}}{ }^{137} \mathrm{Cs} \\
{ }^{233} \mathrm{U}, \mathrm{TRU}\end{array}$ & Unknown & 15,140 & Unknown & Unknown \\
\hline 1.29 Inactive tank (WC-1) & $\begin{array}{l}{ }^{90} \mathrm{Sr} \\
{ }^{137} \mathrm{Cs} \\
\text { TRU }\end{array}$ & $\begin{array}{l}<1.0 \times 10^{1} \text { est. } \\
<1.0 \times 10^{1} \text { est. } \\
<1.0 \times 10^{-1} \text { est. }\end{array}$ & 7,570 & Unknown & Unknown \\
\hline 1.30a Inactive tank (WC-15) & ${ }^{90} \mathrm{Sr},{ }^{137} \mathrm{Cs}, \mathrm{TRU}$ & $<1.0 \times 10^{-1}$ est. & 3,785 & Unknown. & Unknown \\
\hline 1.30b Inactive tank (WC-17) & $\begin{array}{l}{ }^{90} \mathrm{Sr} \\
{ }^{60} \mathrm{Co} \\
{ }^{137} \mathrm{Cs} \\
{ }^{154} \mathrm{Eu} \\
\text { TRU }\end{array}$ & $\begin{array}{l}2.2 \times 10^{-3} \\
2.2 \times 10^{-5} \\
5.2 \times 10^{-4} \\
1.1 \times 10^{-5} \\
1.2 \times 10^{-3}\end{array}$ & 3,785 & 303 & 3,596 \\
\hline
\end{tabular}




\begin{tabular}{|c|c|c|c|c|c|}
\hline \multirow[b]{2}{*}{ SWMU Site ${ }^{a}$} & \multirow[b]{2}{*}{ Contaminant } & \multirow[b]{2}{*}{$\begin{array}{l}\text { Inventory } \\
{[\mathrm{Ci}(\mathrm{kg})]^{\mathrm{b}}}\end{array}$} & \multicolumn{3}{|c|}{ Current Contents } \\
\hline & & & $\begin{array}{c}\text { Volume } \\
\text { (L) }\end{array}$ & $\begin{array}{r}\text { Sludge } \\
(\mathrm{L})\end{array}$ & $\begin{array}{c}\text { Liquid } \\
\text { (L) }\end{array}$ \\
\hline 1.31a Inactive tank (TH-1) & $\begin{array}{l}{ }^{90} \mathrm{Sr} \\
{ }^{60} \mathrm{Co} \\
{ }^{137} \mathrm{Cs} \\
{ }^{232} \mathrm{Th} \\
\text { TRU }\end{array}$ & $\begin{array}{l}6.0 \times 10^{-1} \\
1.0 \times 10^{-2} \\
5.0 \times 10^{-1} \\
3.0 \times 10^{-6} \\
2.0 \times 10^{-4}\end{array}$ & 9,463 & Unknown & 1,798 \\
\hline $1.31 \mathrm{~b}$ Inactive tank ( $\mathrm{TH}-2)$ & $\begin{array}{l}{ }^{90} \mathrm{Sr},{ }^{137} \mathrm{Cs}, \\
{ }^{202} \mathrm{Th}, \mathrm{TRU}\end{array}$ & Unknown & 9,084 & Unknown & Unknown \\
\hline $1.31 \mathrm{c}$ Inactive tank (TH-3) & $\begin{array}{l}{ }^{90} \mathrm{Sr} \\
{ }^{60} \mathrm{Co} \\
{ }^{137} \mathrm{Cs} \\
{ }^{232} \mathrm{Th} \\
\text { TRU }\end{array}$ & $\begin{array}{l}6.0 \times 10^{-1} \\
5.0 \times 10^{-4} \\
6.0 \times 10^{-1} \\
1.0 \times 10^{-6} \\
2.0 \times 10^{-4}\end{array}$ & 12,491 & Unknown & 2,650 \\
\hline 1.32 Inactive tank $(\mathrm{TH}-4)$ & $\begin{array}{l}{ }^{90} \mathrm{Sr} \\
{ }^{137} \mathrm{Cs} \\
{ }^{232} \mathrm{Th} \\
\text { TRU, U }\end{array}$ & $\begin{array}{l}6.0 \times 10^{-2} \\
5.0 \times 10^{-1} \\
1.0 \times 10^{-5} \\
8.5 \times 10^{-2}\end{array}$ & 53,993 & 20,818 & 37,093 \\
\hline 1.33 Active tank (2026) & Unidentified & Unknown & 1,893 & Unknown & Variable \\
\hline 1.34 Active tank (WC-2) & Unidentified & Unknown & 3,785 & Unknown & Variable \\
\hline 1.35 Active tank (WC-3) & Unidentified & Unknown & 3,785 & Unknown & Variable \\
\hline 1.36 Inactive tank (WC -4 ) & Unidentified & Unknown & 6,435 & Unknown & Unknown \\
\hline 1.37a Active tank (WC-5) & LLW & Unknown & 3,785 & Unknown & Variable \\
\hline 1.37b Active tank (WC-6) & LLW & Unknown & 18,925 & Unknown & Variable \\
\hline 1.37c Active tank (WC-8) & LLW & Unknown & 3,785 & Unknown & Variable \\
\hline 1.37d Active tank (WC-9) & LLW & Unknown & 8,100 & Unknown & Variable \\
\hline 1.38 Active tank (WC-7) & LLW & Unknown & 4,164 & Unknown & Variable \\
\hline 1.39a Active tank (WC-10) & LLW & Unknown & 8,706 & Unknown & Variable \\
\hline 1.39b Active tank (WC-11) & LLW & Unknown & 17,411 & Unknown & Variable \\
\hline 1.39c Active tank (WC-12) & LLW & Unknown & 3,785 & Unknown & Variable \\
\hline 1.39d Active tank (WC-13) & LLW & Unknown & 3,785 & Unknown & Variable \\
\hline $1.39 \mathrm{e}$ Active tank (WC-14) & LLW & Unknown & 3,785 & Unknown & Variable \\
\hline 1.40 Active tank (WC-19) & LLW & Unknown & 7,949 & Unknown & Variable \\
\hline 1.41 Active tank (W-12) & LLW & Unknown & 2,650 & Unknown & Variable \\
\hline 1.42a Active tank (W-16) & LLW & Unknown & 3,785 & Unknown & Variable \\
\hline 1.42b Active tank (W-17) & LLW & Unknown & 3,785 & Unknown & Variable \\
\hline
\end{tabular}




\begin{tabular}{|c|c|c|c|c|c|}
\hline \multirow[b]{2}{*}{ SWMU Site ${ }^{\mathrm{a}}$} & \multirow[b]{2}{*}{ Contaminant } & \multirow[b]{2}{*}{$\begin{array}{l}\text { Inventory } \\
{[\mathrm{Ci}(\mathrm{kg})]}\end{array}$} & \multicolumn{3}{|c|}{ Current Contents } \\
\hline & & & $\begin{array}{l}\text { Volume } \\
\text { (L) }\end{array}$ & $\begin{array}{l}\text { Sludge } \\
\text { (L) }\end{array}$ & $\begin{array}{l}\text { Liquid } \\
\text { (L) }\end{array}$ \\
\hline 1.42c Active tank (W-18) & LLW & Unknown & 3,785 & Unknown & Variable \\
\hline 1.46 SWSA 1 (2624) & $\begin{array}{l}{ }^{90} \mathrm{Sr}, \\
\text { Unidentified } \mathrm{Hz}^{\mathrm{h}}\end{array}$ & $\begin{array}{l}<4.0 \times 10^{3} \\
\text { Unknown }\end{array}$ & Unknown & N/A & N/A \\
\hline 1.47 SWSA 2 (4003) & Unidentified & $\begin{array}{l}\text { Presence } \\
\text { unconfirmed } \\
\text { Contents moved } \\
\text { to SWSA } 3 \\
\text { before } 1950\end{array}$ & Unknown & N/A & N/A \\
\hline $\begin{array}{l}1.54 \text { Waste oil storage tanks } \\
\text { (2525) (2 tanks) }\end{array}$ & Unidentified & Unknown & 3,785 & Unknown & Unknown \\
\hline 1.56a Inactive tank (W-19) & Nitric acid waste & Unknown & 8,516 & Empty & Empty \\
\hline 1.56b Inactive tank (W-20) & Nitric acid waste & Unknown & 8,516 & Empty & Empty \\
\hline $\begin{array}{l}1.58 \text { Former Waste Pile } \\
\text { Area }\end{array}$ & Construction debris & Unknown & N/A & N/A & N/A \\
\hline $\begin{array}{l}\text { 1.62 Waste Evaporator Facility } \\
(3506)\end{array}$ & $\mathrm{LLW},{ }^{137} \mathrm{CS},{ }^{90} \mathrm{Sr}$ & Unknown & N/A & N/A & N/A \\
\hline $\begin{array}{l}1.63 \text { Storage canal and } \\
\text { dissolver pit (3505) }\end{array}$ & LLW & Unknown & N/A & N/A & N/A \\
\hline
\end{tabular}

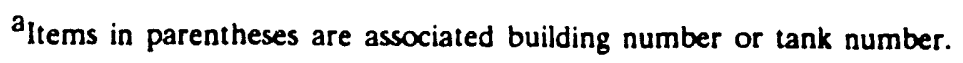

${ }^{b}$ Numbers in parentheses are measured in kilograms; all others are in curies.

${ }^{c} \mathrm{~N} / \mathrm{A}=$ Not Applicable.

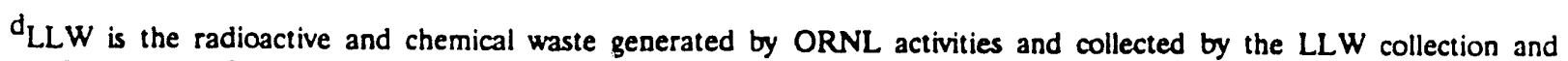
transfer system. No routine effort has been made to determine the composition of the waste stream. It has been estimated that the average activity is about $114 \mathrm{mCi} / \mathrm{L}$ and the major radionuclides present are ${ }^{90} \mathrm{Sr},{ }^{137} \mathrm{Cs},{ }^{60} \mathrm{Co}$, and various rare earths, with some plutonium, uranium, and TRU isotopes present.

e Impoundment has been filled in.

$\mathrm{f}_{\text {Liquid }}$ and sediments.

gInactive refers to tanks no longer receiving new waste additions; most are still storing liquid wastes and/or sludges. Active refers to tanks that are in use for waste collection and storage of newly generated wastes.

$h_{\mathrm{Hz}}$ refers to hazardous wastes.

$1 \mathrm{~Bq}=27.03 \mathrm{pCi}$

$1 \mathrm{Ci}=10^{12} \mathrm{pCi}$ 


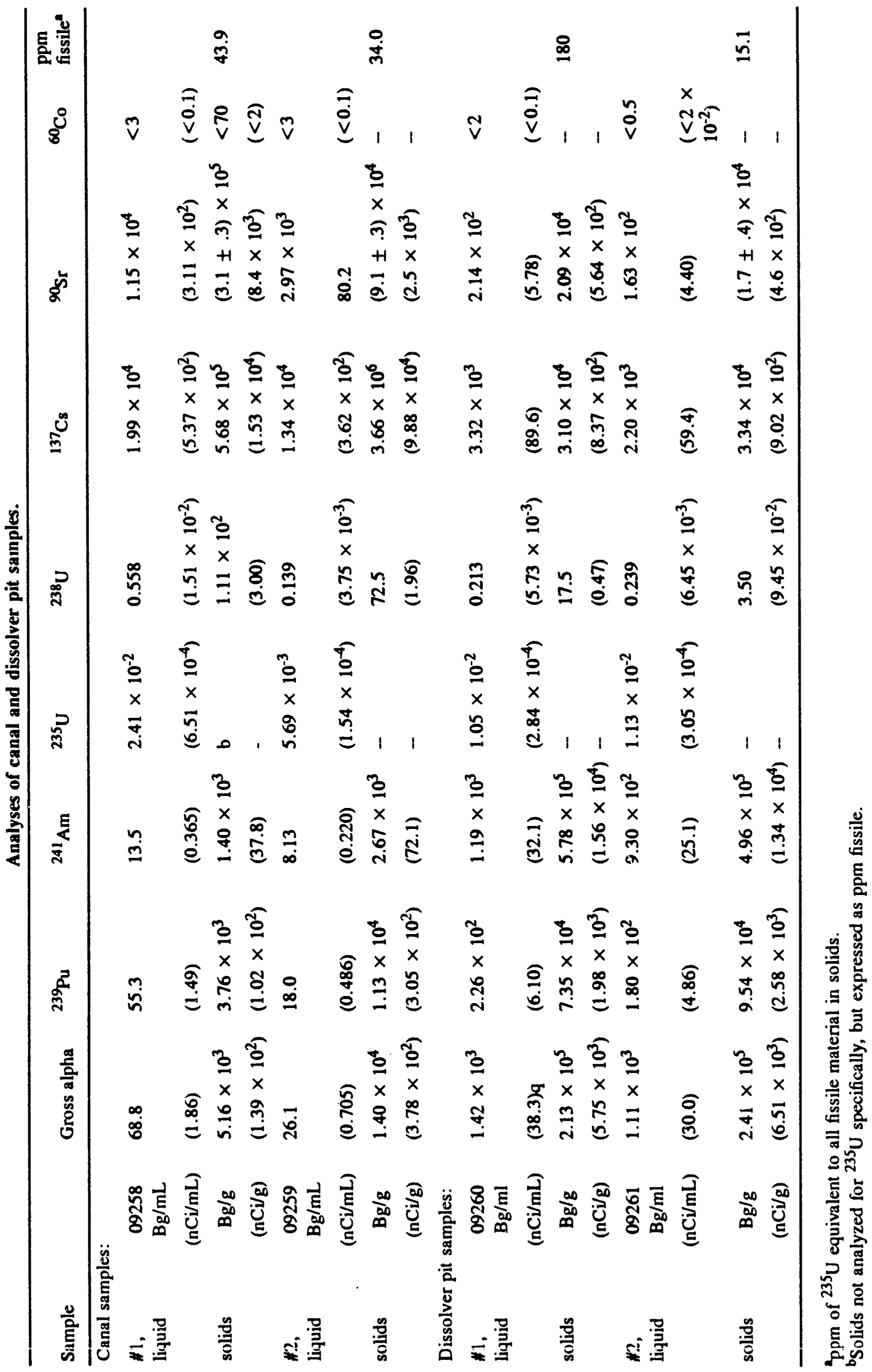




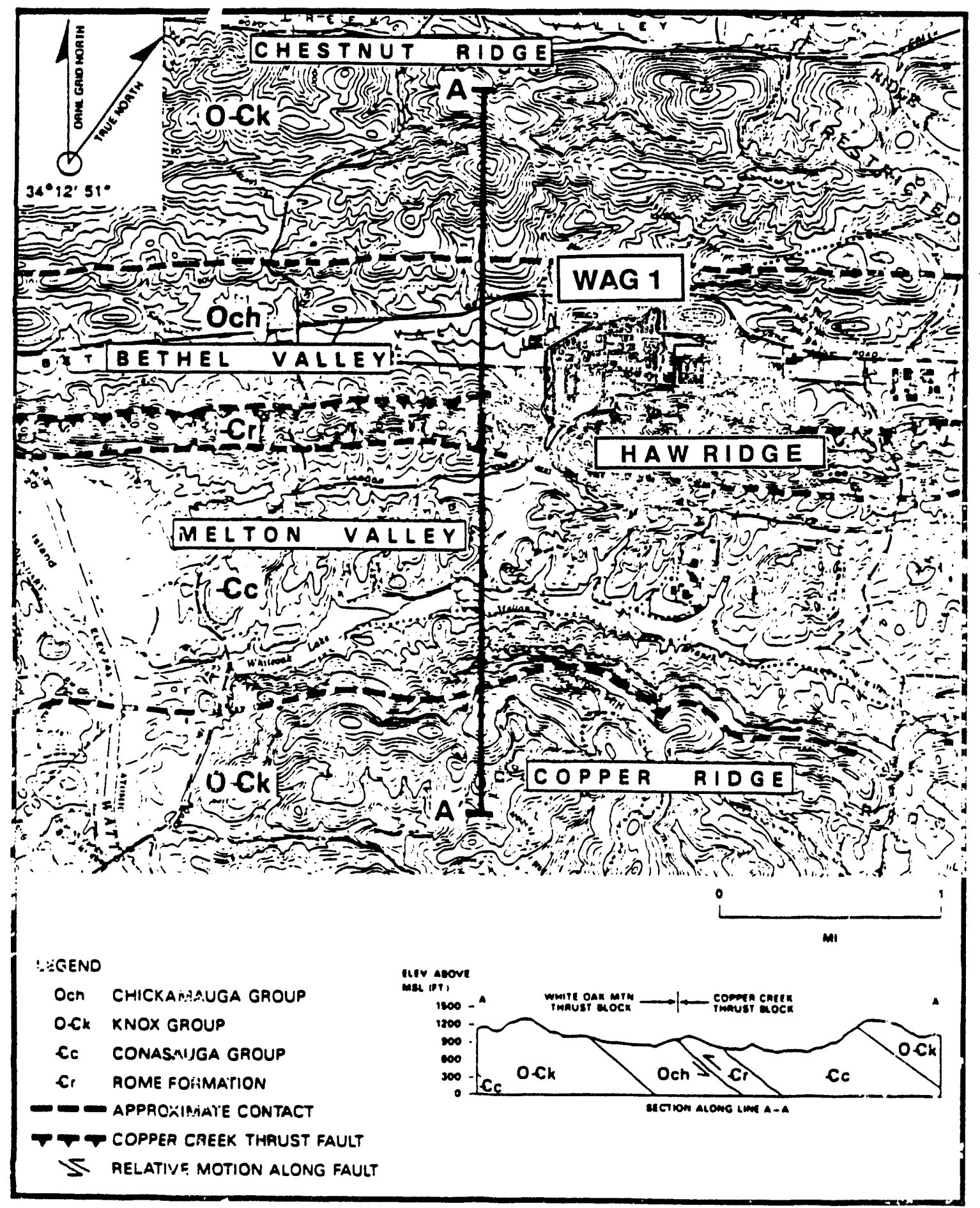

Geologic map of the ORNL area. Source: W. M. McMaster, Geologic Map of the Oak Ridge Reservation, Tennessee, ORNL/TM-713, Oak Ridge National Laboratory, 1963. 
Attachment A, CERCLA Specific Comment Response 66

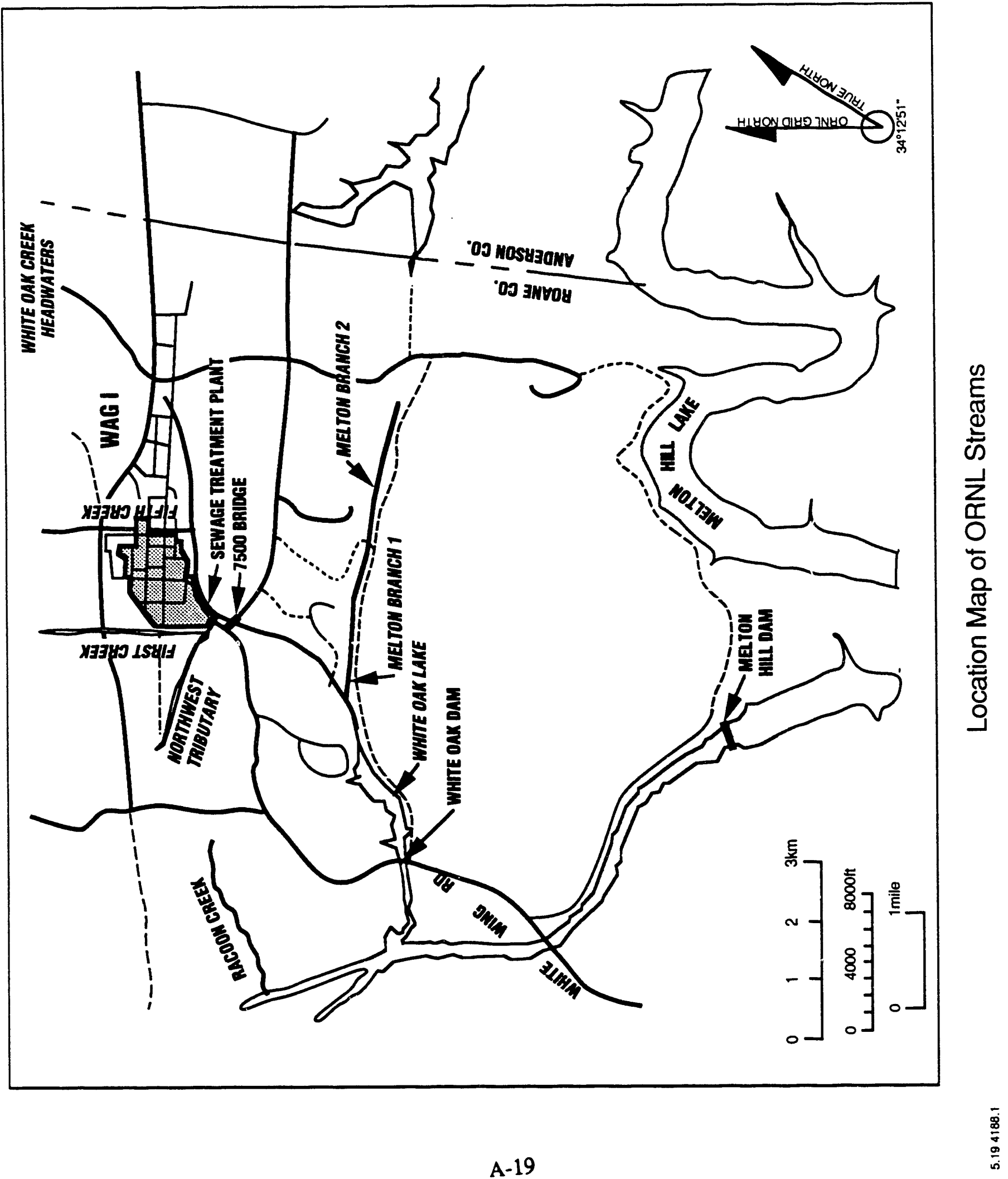




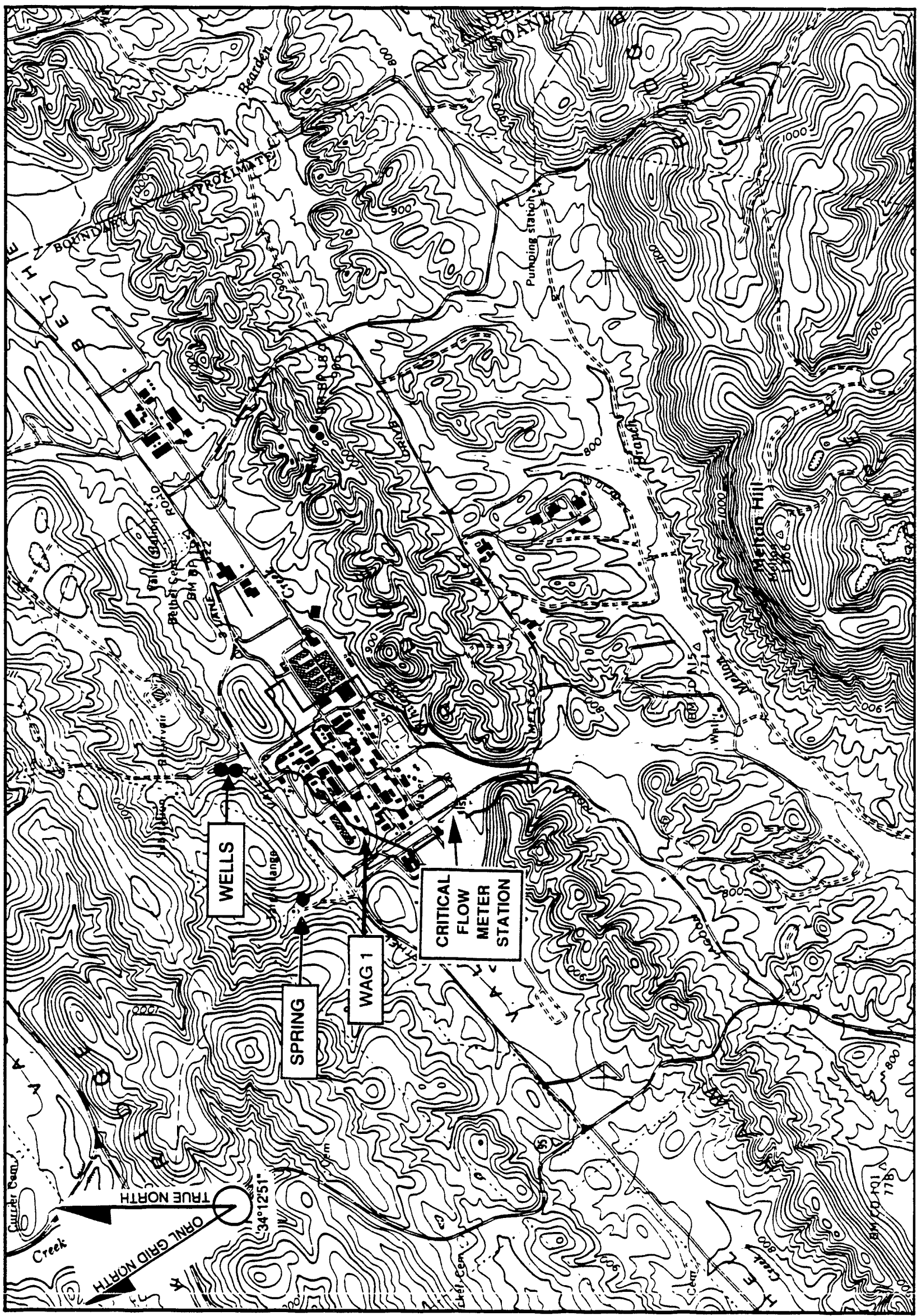

总]

ฮั

은

을

(1)

임

ธั

$\frac{1}{0}$

$\stackrel{0}{\mathcal{0}}$

㐫 
Attachment A, CERCLA Specific Comment Response 70

Radionuclide concentrations in surface water and NPDES stations around ORNL in 1989

Concentration

\begin{tabular}{|c|c|c|c|c|c|}
\hline \multirow[b]{2}{*}{ Radionuclide } & \multirow[b]{2}{*}{ No. of samples } & \multicolumn{4}{|c|}{$\begin{array}{l}\text { Concentration } \\
(\mathrm{pCi} / \mathrm{L})\end{array}$} \\
\hline & & $\operatorname{Max}$ & Min & Av & Std. error ${ }^{\mathrm{a}}$ \\
\hline \multicolumn{6}{|c|}{7500 bridge } \\
\hline${ }^{60} \mathrm{Co}$ & 12 & 54 & -24 & 13 & 6.1 \\
\hline${ }^{137} \mathrm{Cs}$ & 12 & 180 & -5.4 & 70 & 14 \\
\hline Total $\mathbf{S r}^{\mathbf{b}}$ & 12 & 240 & 49 & 88 & 16 \\
\hline${ }^{3} \mathbf{H}$ & 12 & 24000 & 2400 & 7700 & 1700 \\
\hline \multicolumn{6}{|c|}{ First Creek } \\
\hline${ }^{60} \mathrm{Co}$ & 12 & 57 & -7.6 & 8.4 & 5.3 \\
\hline${ }^{137} \mathrm{Cs}$ & 12 & 51 & -22 & 5.6 & 5.3 \\
\hline Total Sr ${ }^{b}$ & 12 & 510 & 190 & 310 & 31 \\
\hline \multicolumn{6}{|c|}{ Fifth Creek } \\
\hline${ }^{60} \mathrm{Co}$ & 12 & 51 & -14 & 10 & 4.8 \\
\hline${ }^{137} \mathrm{Cs}$ & 12 & 14 & -27 & -6.4 & 3.5 \\
\hline Total $\mathrm{Sr}^{\mathrm{b}}$ & 12 & 54 & 1.4 & 34 & 3.5 \\
\hline \multicolumn{6}{|c|}{1500 area (X03) } \\
\hline Gross alpha & 4 & 14 & 2.4 & 7.5 & 2.7 \\
\hline Gross beta & 4 & 22 & 0.0 & 12 & 5.1 \\
\hline \multicolumn{6}{|c|}{2000 area $(X 04)$} \\
\hline${ }^{60} \mathrm{Co}$ & 4 & 5.4 & -8.1 & -0.41 & 2.9 \\
\hline${ }^{137} \mathrm{Cs}$ & 4 & 35 & -19 & 0.068 & 12 \\
\hline Gross beta & 4 & 59 & 0.0 & 26 & 12 \\
\hline Total $\mathbf{S} \mathbf{r}^{\mathbf{b}}$ & 4 & 11 & -0.27 & 4.1 & 2.6 \\
\hline \multicolumn{6}{|c|}{190 ponds (X06) } \\
\hline${ }^{60} \mathrm{Co}$ & 4 & 22 & -24 & -3.6 & 9.5 \\
\hline${ }^{137} \mathrm{Cs}$ & 4 & 41 & -2.7 & 12 & 9.7 \\
\hline Gross alpha & 4 & 30 & -2.7 & 13 & 6.7 \\
\hline Gross beta & 4 & 46 & 32 & 38 & 2.9 \\
\hline \multicolumn{6}{|c|}{190 ponds, 1500 Area and 2000 Area (X06A) } \\
\hline${ }^{60} \mathrm{Co}$ & 8 & 24 & -32 & -4.0 & 6.2 \\
\hline${ }^{137} \mathrm{Cs}$ & 8 & 24 & -8.1 & 10 & 3.5 \\
\hline Gross alpha & 8 & 16 & -5.4 & 5.4 & 2.1 \\
\hline Gross beta & 8 & 81 & -11 & 27 & 11 \\
\hline Total $\mathbf{S r}^{\mathrm{b}}$ & 8 & 17 & 2.4 & 8.1 & 1.6 \\
\hline
\end{tabular}

aStandard error of the mean.

${ }^{b}$ Total radioactive $\mathrm{Sr}\left({ }^{89} \mathrm{Sr}+{ }^{90} \mathrm{Sr}\right)$. 
Attachment A, CERCLA Specific Comment Response 75

Concentration of mercury in sediment samples collected from WAG 1 in 1979 and 1983

\begin{tabular}{lcc}
$\begin{array}{l}\text { Sampling } \\
\text { location }\end{array}$ & $\begin{array}{c}\text { Concentration } \\
\text { (ppm) }\end{array}$ \\
\hline & 1979 & \\
P-6 & & 0.08 \\
T-8 & & 0.41 \\
T-10 & 1983 & 3.8 \\
& & \\
6-LT & & 2.9 \\
6-LM & & 2.0 \\
6-LB & 6.4 \\
6-RT & 9.5 \\
6-RM & 1.6 \\
6-RB & 2.8 \\
7-RT & 5.1 \\
7-RM & 18 \\
7-RB & 19 \\
7-LT & 8.1 \\
7-LB & 0.4 \\
8-RT & 2.5 \\
8-RM & 4.5 \\
8-RB & 1.4 \\
& \\
\hline & & \\
\hline
\end{tabular}

${ }^{a} R, M$, and $L$ indicate right, middle, and left of stream looking downstream. $T, M$ and $B$ indicate top, middle, and bottom of a 10 -in. core. 
Summary of parameters detected in groundwater from 42 piezometers in WAG 1 during scoping survey by Ketelle

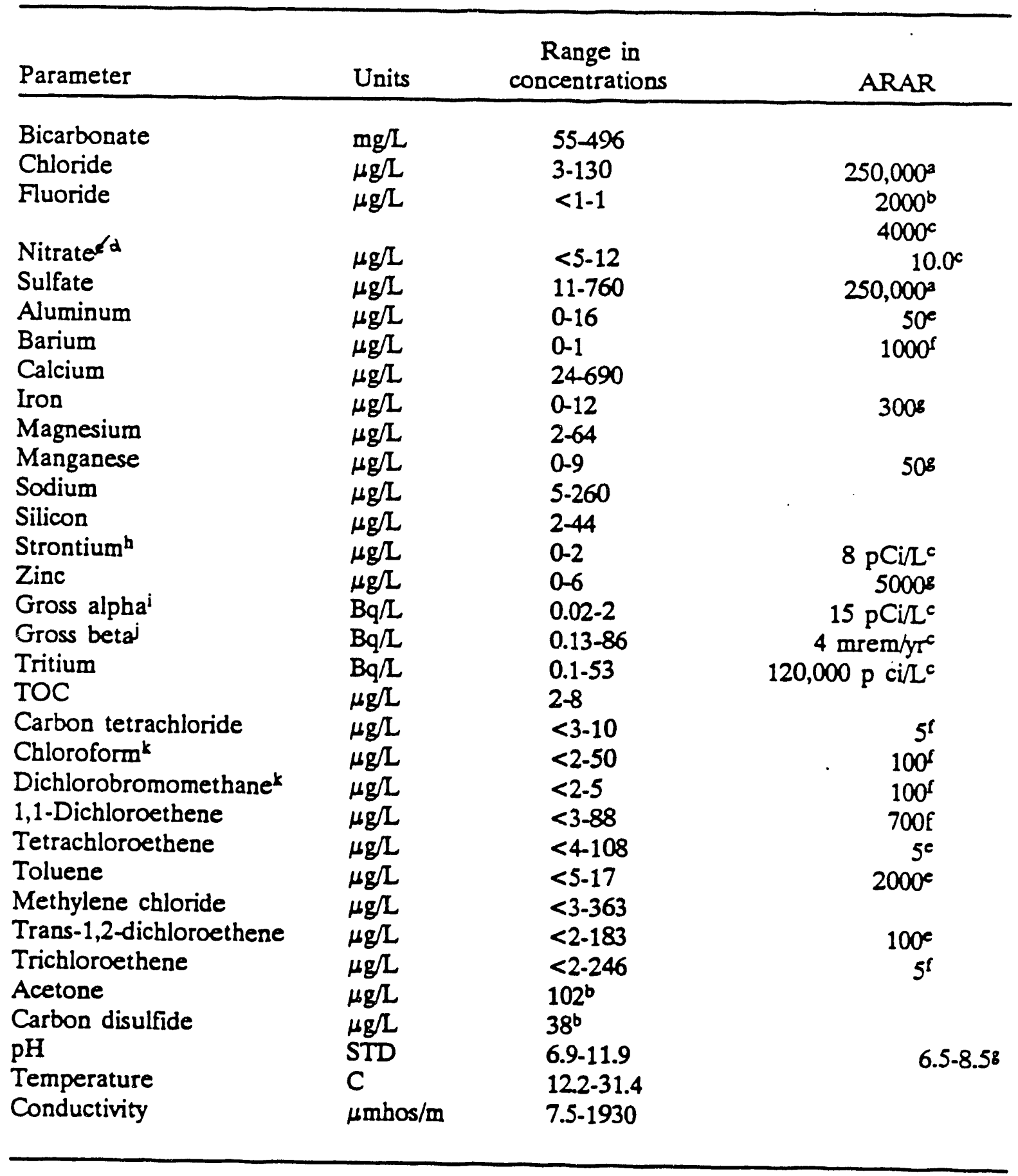

Tennessee Department of Health and Environment (TDHE). State Secondary Drinking Water Regulation. Chapter 1200-5-1, as amended November 10, 1988.

WTDHE State Water Quality Standard, MCL. Chapter 1200-4-3. 
TDHE. State Primary Drinking Water Standard, MCL Chapter 1200-5-1.

dValue is that for nitrate as nitrogen. MCL for nitrate is applicable to both community water systems and noncommunity water systems.

'Federal Register 54, 22062. Proposed Federal Secondary Drinking Water MCL. May $22,1989$.

'Code of Federal Regulations, Part 40, Subpart 141. National Primary Drinking Water Standard, MCL.

¿Code of Federal REgulations, Part 40, Subpart 141. National Secondary Drinking Water Standard, MCL.

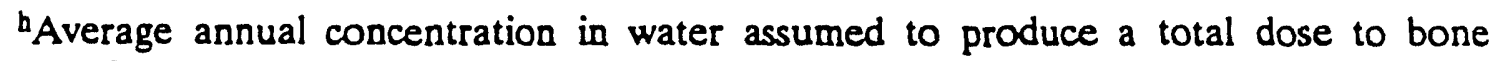
marrow of 4 mrem/yr.

Includes RAdium-226, but excludes Radon and Uranium

jChapter 1200-5-1.06 of the rules of the TDHE specifies that if two or more radionuclides are present, the sum of their annual dose equivalent to the total body or to any organ shall not exceed 4 mrem/yr. Guidance on calculation of average annual concentrations assumed to produce a total body dose of 4 mrem/yr is given.

Value is that for total trihalomethanes.

'Only one well sampled for these parameters.

Source: L. Voorhees, Oak Ridge National Laboratory, Letter to P. Bengel, BNI Team, BNI CCN 000233, Oak Ridge, Tenn., 1987. 


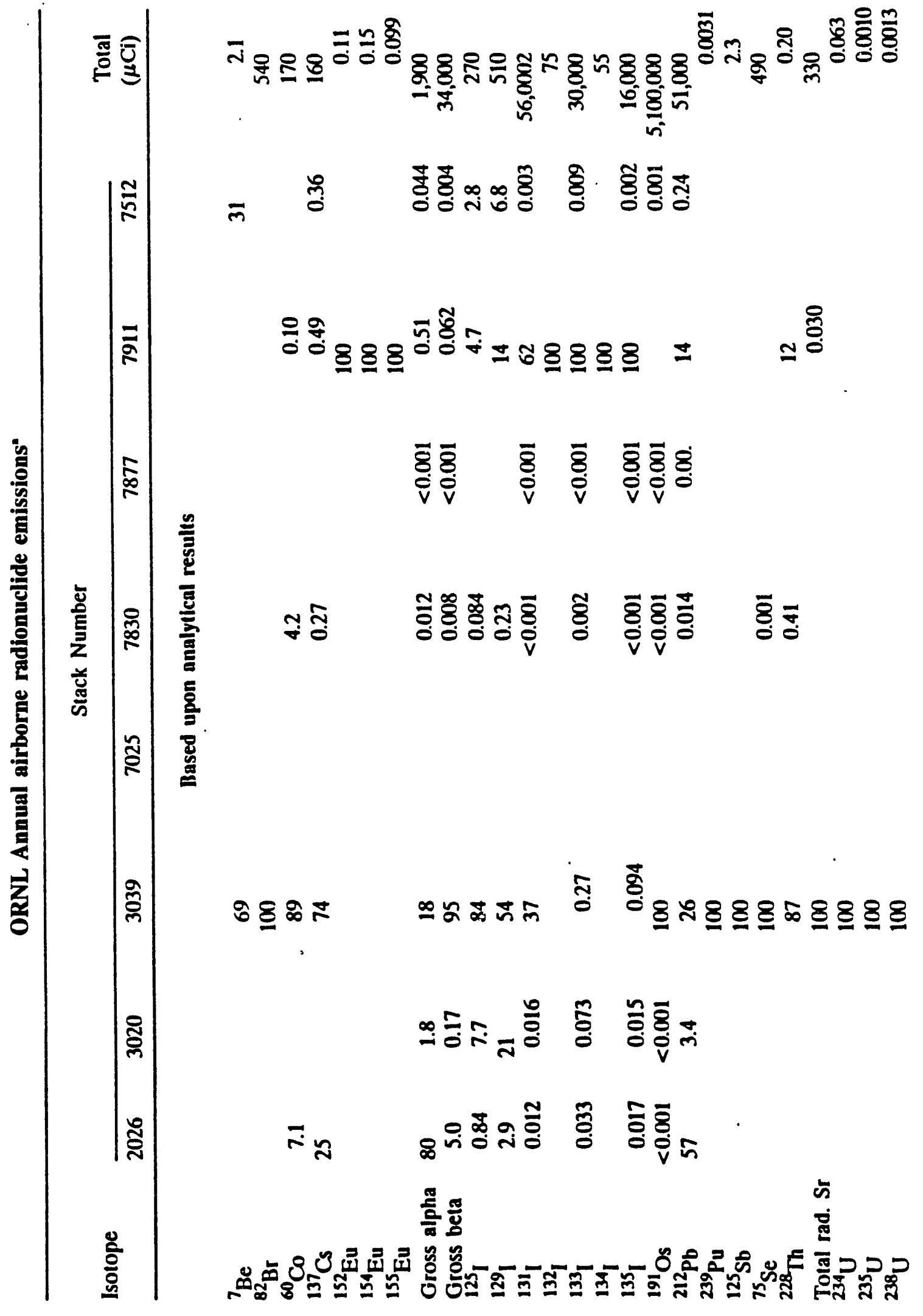


Attachment A, CERCLA Specific Comment Response 90

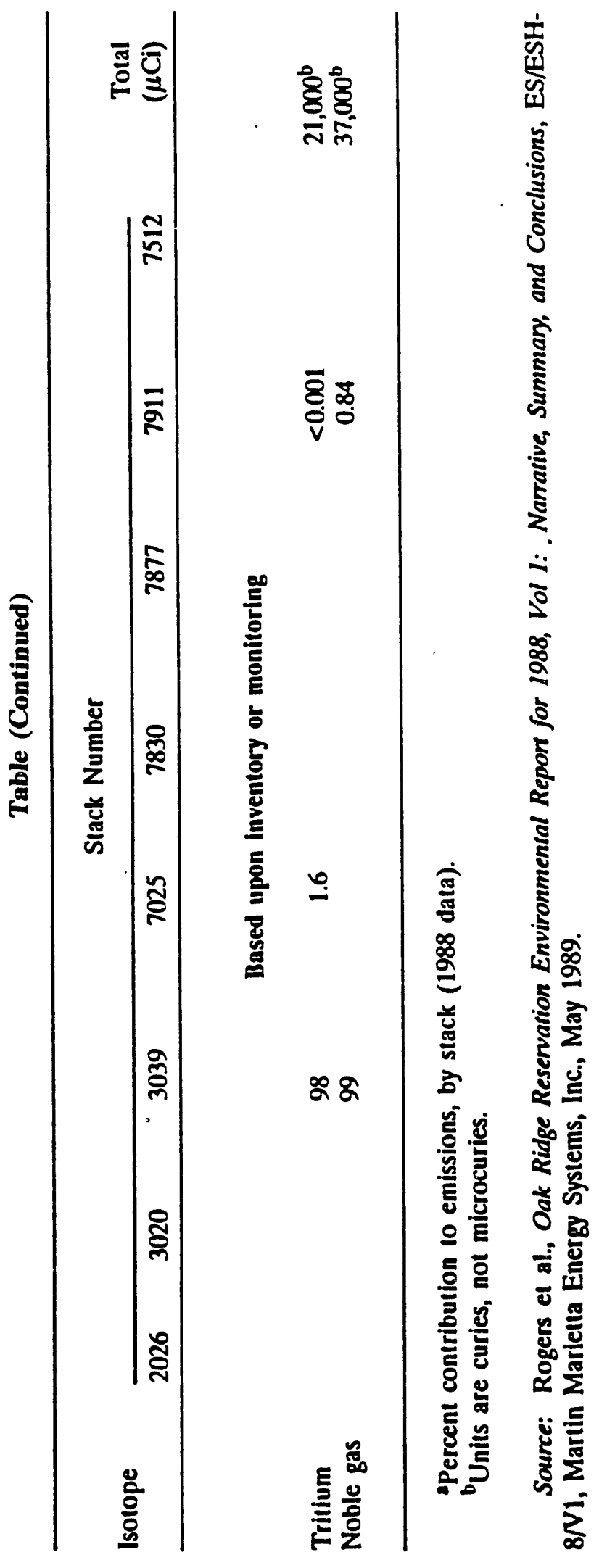


Attachment A, CERCLA Specific Comment Response 94

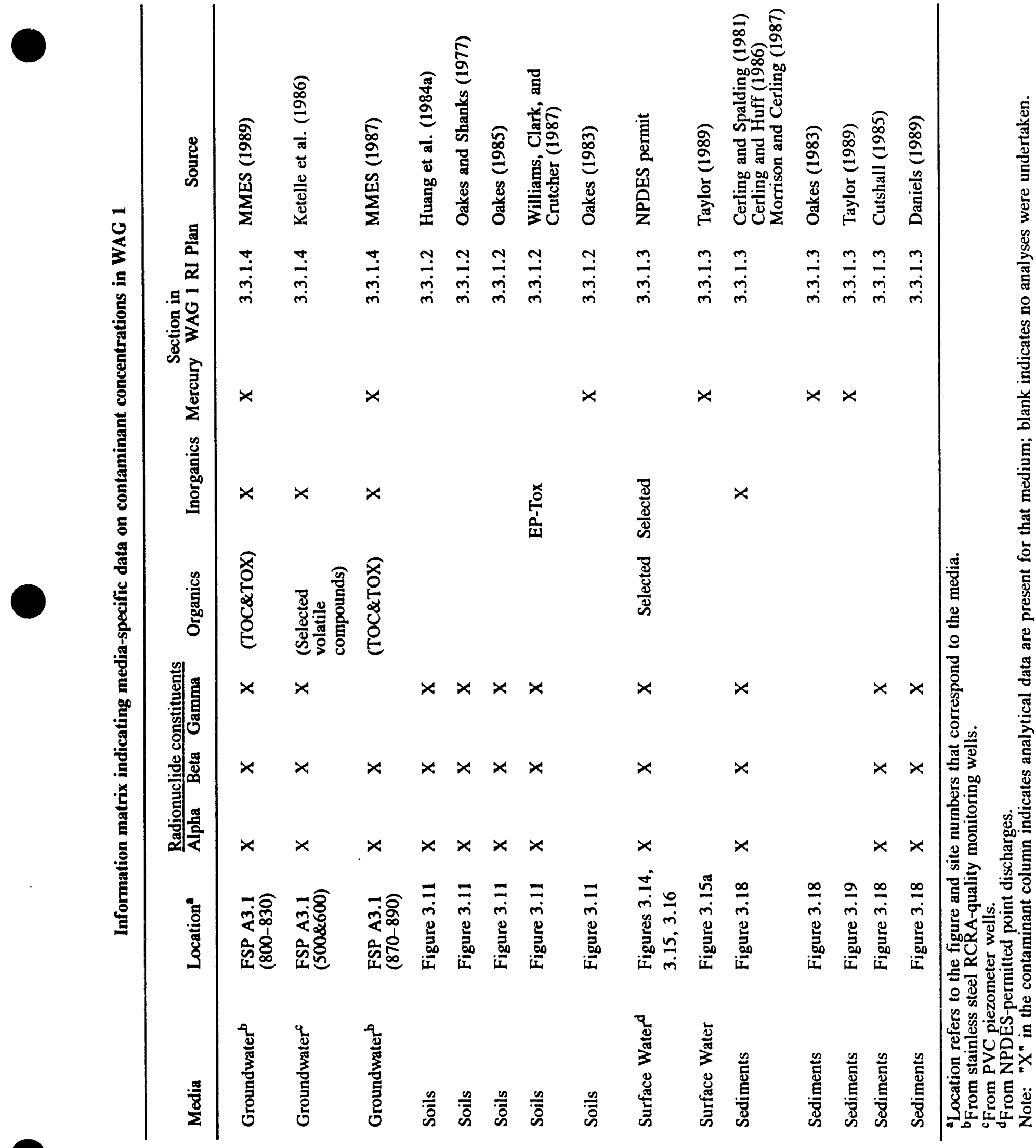


Attachment A, CERCLA Specific Comment Response 94

Maximum concentrations in sampled perimeter monitoring wells October-December 1988

\begin{tabular}{|c|c|c|c|}
\hline Parameter & Well & Concentration & Units \\
\hline Gross alpha & 812 & 8.6 & $\mathrm{~Bq} / \mathrm{L}$ \\
\hline Gross beta & 812 & 660 & $\mathrm{~Bq} / \mathrm{L}$ \\
\hline${ }^{60} \mathrm{Co}$ & 809 & 0.24 & $\mathrm{~Bq} / \mathrm{L}$ \\
\hline${ }^{137} \mathrm{Cs}$ & 807 & 0.22 & $\mathrm{Bg} / \mathrm{L}$ \\
\hline Sr (total radiation) & 812 & 280 & $\mathrm{~Bq} / \mathrm{L}$ \\
\hline $\mathrm{Ra}$ (total) & 813 & 0.09 & $\mathrm{~Bq} / \mathrm{L}$ \\
\hline Tritium & 830 & 2000 & $\mathrm{~Bq} / \mathrm{L}$ \\
\hline $\mathrm{Al}$ & 813,828 & 2.1 & $\mathrm{mg} / \mathrm{L}$ \\
\hline As & 811 & 0.05 & $\mathrm{mg} / \mathrm{L}$ \\
\hline $\mathrm{Be}$ & 807 & 0.003 & $\mathrm{mg} / \mathrm{L}$ \\
\hline B & 811 & 0.77 & $\mathrm{mg} / \mathrm{L}$ \\
\hline $\mathrm{Ca}$ & 830 & 200 & $\mathrm{mg} / \mathrm{L}$ \\
\hline $\mathrm{Cl}$ & 808 & 79 & $\mathrm{mg} / \mathrm{L}$ \\
\hline $\mathrm{Fe}$ & 809 & 14 & $\mathrm{mg} / \mathrm{L}$ \\
\hline $\mathrm{Mg}$ & 819 & 29 & $\mathrm{mg} / \mathrm{L}$ \\
\hline $\mathrm{Mn}$ & 830 & 6.5 & $\mathrm{mg} / \mathrm{L}$ \\
\hline $\mathrm{Ni}$ & 824 & 0.026 & $\mathrm{mg} / \mathrm{L}$ \\
\hline $\mathrm{Na}$ & 811 & 258 & $\mathrm{mg} / \mathrm{L}$ \\
\hline $\mathrm{Ti}$ & 828 & 0.056 & $\mathrm{mg} / \mathrm{L}$ \\
\hline $\mathrm{V}$ & 816 & 0.016 & $\mathrm{mg} / \mathrm{L}$ \\
\hline $\mathrm{Zn}$ & 813 & 0.37 & $\mathrm{mg} / \mathrm{L}$ \\
\hline Fecal coliform & 822,827 & 3 & $\mathrm{COL} / 100 \mathrm{~mL}$ \\
\hline Fluroide & 811 & 2.9 & $\mathrm{mg} / \mathrm{L}$ \\
\hline Nitrate & 828 & 2.2 & $\mathrm{mg} / \mathrm{L}$ \\
\hline Sulfate (as $\mathrm{SO}_{4}$ ) & 830 & 250 & $\mathrm{mg} / \mathrm{L}$ \\
\hline TOC & 809 & 4.4 & $\mathrm{mg} / \mathrm{L}$ \\
\hline Organic halides (total) & 825 & 0.16 & $\mathrm{mg} / \mathrm{L}$ \\
\hline $\mathrm{pH}(\min )$ & 827 & 6.7 & STD units \\
\hline$(\max )$ & 811 & 9.2 & STD units \\
\hline Temperature & 823 & $29-31$ & degrees $\mathrm{C}$ \\
\hline
\end{tabular}


Attachment A, CERCLA Specific Comment Response 94

Concentrations of parameters in wells around 3539-40 analyzed in $1985-1986$

\begin{tabular}{|c|c|c|c|c|c|c|}
\hline \multirow[b]{2}{*}{ Radionuclides } & \multirow{2}{*}{$\begin{array}{l}\text { Number of } \\
\text { samples }\end{array}$} & \multicolumn{3}{|c|}{ Concentrations } & \multirow[b]{2}{*}{$95 \% \mathrm{CC}^{\mathrm{a}}$} & \multirow[b]{2}{*}{ ARAR } \\
\hline & & $\operatorname{Max}$ & Min & Av & & \\
\hline 2,4,5-TP Silvex & 14 & $<0.01$ & $<0.01$ & $<0.01$ & 0 & $0.01^{b}$ \\
\hline 2,4-D & 14 & 0.06 & $<0.01$ & $<0.014$ & 0 & $0.1^{b}$ \\
\hline $\mathrm{Ag}$ & 14 & $<0.005$ & $<0.005$ & $<0.005$ & 0 & $0.05^{b}$ \\
\hline As & 14 & $<0.01$ & $<0.01$ & $<0.01$ & 0 & $0.05^{b}$ \\
\hline $\mathrm{Ba}$ & 14 & $<1.0$ & $<1.0$ & $<1.0$ & 0 & $1.0^{\mathrm{b}}$ \\
\hline $\mathrm{Cd}$ & 14 & $<0.002$ & $<0.002$ & $<0.002$ & 0 & $0.010^{\mathrm{b}}$ \\
\hline $\mathrm{Cl}$ & 14 & 17 & 5.2 & 8.2 & 1.7 & $250^{c}$ \\
\hline $\mathrm{Cr}^{\mathrm{d}}$ & 14 & 0.032 & $<0.02$ & $<0.021$ & 0.0017 & $0.05^{b}$ \\
\hline Endrin & 14 & $<0.0002$ & $<0.0002$ & $<0.0002$ & 0 & $0.0002^{b}$ \\
\hline F & 14 & $<1.0$ & $<1.0$ & $<1.0$ & 0 & $\begin{array}{l}2000^{e} \\
4000^{f}\end{array}$ \\
\hline $\mathrm{Fe}$ & 14 & 5.9 & 0.052 & 1.8 & 0.84 & $300^{c}$ \\
\hline Fecal coliform 8 & 14 & 0 & 0 & 0 & 0 & $\begin{array}{l}1000^{\mathrm{e}} \\
1.0^{\mathrm{f}}\end{array}$ \\
\hline Gross alpha ${ }^{\mathrm{b}, \mathrm{i}}$ & 14 & 0.52 & 0.03 & 0.23 & 0.0023 & $15 \mathrm{pCi} / \mathrm{L}^{\mathrm{f}}$ \\
\hline Gross beta ${ }^{h, j}$ & 14 & 2.0 & 0.081 & 0.74 & 0.01 & $4 \mathrm{mrem} / \mathrm{yr}^{\mathrm{f}}$ \\
\hline $\mathrm{Hg}$ & 14 & $<0.0001$ & $<0.0001$ & $<0.000$ & 10.0 & $0.002^{b}$ \\
\hline Lindane & 14 & $<0.002$ & $<0.002$ & $<0.002$ & 0 & $0.004^{b}$ \\
\hline Methoxychlor & 14 & $<0.008$ & $<0.008$ & $<0.008$ & 0 & $0.01^{b}$ \\
\hline $\mathrm{Mn}$ & 14 & 10 & 0.01 & 4.4 & 2.0 & $50^{c}$ \\
\hline $\mathrm{Na}$ & 14 & 220 & 4.8 & 26 & 31 & \\
\hline $\mathrm{NO}_{3}{ }^{\mathrm{k}}$ & 14 & $<5.0$ & $<5.0$ & $<5.0$ & 0 & $10^{f}$ \\
\hline $\mathrm{Pb}$ & 14 & 1.2 & 0.020 & 0.10 & 0.17 & $0.05^{b}$ \\
\hline $\mathrm{pH}^{\prime}$ & 98 & 13 & 6.5 & 7.6 & 0.29 & $6-9^{e}$ \\
\hline Phenols & 14 & 0.003 & $<0.001$ & $<0.002$ & 0.0004 & \\
\hline $\mathbf{R a}(\text { total })^{\mathbf{h}, \mathbf{m}}$ & 14 & 0.17 & 0.011 & 0.03 & 0.0007 & $5 \mathrm{pCi} / \mathrm{L}^{\mathrm{f}}$ \\
\hline $\mathrm{Se}$ & 14 & $<0.005$ & $<0.005$ & $<0.005$ & 0 & $0.01^{b}$ \\
\hline $\mathrm{SO}_{4}$ & 14 & 250 & $<5.0$ & $<6.5$ & 39 & $250^{\mathrm{c}}$ \\
\hline Specific conductance ${ }^{n}$ & 98 & 1.0 & 0.01 & 0.38 & 0.044 & \\
\hline Temperature $^{o}$ & 98 & 20 & 13 & 16 & 0.26 & \\
\hline Total organic carbon & 56 & 23 & 1.6 & 5.1 & 1.4 & \\
\hline Total organic halides & 56 & 0.093 & $<0.005$ & $<0.005$ & 0 & \\
\hline Toxaphene & 4 & $<0.005$ & $<0.005$ & $<0.005$ & 0 & $0.005^{b}$ \\
\hline
\end{tabular}

a5\% confidence coefficient (CC) about the average.

${ }^{b}$ Code of Federal Regulations, Part 40, Subpart 141, National Primary Drinking Water Maximum Concentration Limits (MCL).

'Tennessee Department of Health and Environment (TDHE), State Secondary Drinking Water Regulation, Chapter 1200-5-1, as amended November 10, 1988.

${ }^{\mathrm{M}} \mathrm{MCL}$ listed is that for total chromium, but is based on the toxicity value for chromium VI.

oTDHE, State Water Quality Standard MCL, Chapter 1200-4-3. 


\section{Attachment A, CERCLA Specific Comment}

Response 94

fTDHE, State Primary Drinking Water MCL, Chapter 1200-5-1, as amended November 10, 1988.

UUnits are colonies per 100 milliliters.

bunits are becquerels per liter.

${ }^{i}$ Includes ${ }^{226} \mathrm{Ra}$, but excludes radon and uranium.

jChapter 122-5-1.06 of the rules of the TDHE specifies that if two or more radionuclides are present, the sum of their annual dose equivalent to the total body or to any organ shall not exceed $4 \mathrm{mrem} /$ year. Guidance on calculation of average annual concentrations assumed to produce a total body dose of $4 \mathrm{mrem} / \mathrm{year}$ is given.

kValue is that for nitrate as nitrogen. MCL for nitrate is applicable to both community water systems and noncommunity water systems.

'Value in pH units.

${ }^{\mathrm{m}}$ Combined ${ }^{226} \mathrm{Ra}$ and ${ }^{228} \mathrm{Ra}$.

${ }^{n}$ Units are micromhos per centimeter.

${ }^{\circ}$ Units are degrees Celsius.

Source: T. W. Oakes et al., Environmental Surveillance of the U.S. Department of Energy Oak Ridge Reservation and Surrounding Environs During 1986, ES/ESH-1/V1, Martin Marietta Energy Systems, Inc., Oak Ridge National Laboratory, 1987. 


\section{Attachment A, CERCLA Specific Comment Response 94}

Concentrations of parameters in wells around 3524 analyzed in 1985-1986

\begin{tabular}{|c|c|c|c|c|c|c|}
\hline \multirow[b]{2}{*}{ Radionuclides } & \multirow{2}{*}{$\begin{array}{l}\text { Number of } \\
\text { samples }\end{array}$} & \multicolumn{4}{|c|}{ Concentrations } & \multirow[b]{2}{*}{ ARAR } \\
\hline & & $\operatorname{Max}$ & Min & Av & $95 \% \mathrm{CC}^{\mathrm{a}}$ & \\
\hline 2,4,5-TP Silvex & 10 & $<0.01$ & $<0.01$ & $<0.01$ & 0 & $0.01^{\mathrm{b}}$ \\
\hline $2,4-D$ & 10 & 0.01 & $<0.01$ & $<0.01$ & 0 & $0.1^{b}$ \\
\hline $\mathrm{Ag}$ & 10 & $<0.005$ & $<0.005$ & $<0.005$ & 0 & $0.05^{b}$ \\
\hline As & 10 & $<0.01$ & $<0.01$ & $<0.01$ & 0 & $0.05^{b}$ \\
\hline $\mathrm{Ba}$ & 10 & $<1.0$ & $<1.0$ & $<1.0$ & 0 & $1.0^{\mathrm{b}}$ \\
\hline $\mathrm{Cd}$ & 10 & $<0.002$ & $<0.002$ & $<0.002$ & 0 & $0.010^{\mathrm{b}}$ \\
\hline $\mathrm{Cl}$ & 10 & 11 & 4.7 & 7.0 & 1.3 & $250^{c}$ \\
\hline $\mathrm{Cr}^{\mathrm{d}}$ & 10 & $<0.02$ & $<0.02$ & $<0.020$ & 0 & $0.05^{b}$ \\
\hline Endrin & 10 & $<0.0002$ & $<0.0002$ & $<0.0002$ & 0 & $0.0002^{b}$ \\
\hline $\mathbf{F}$ & 10 & $<1.0$ & $<1.0$ & $<1.0$ & 0 & $\begin{array}{l}2000^{e} \\
4000^{f}\end{array}$ \\
\hline $\mathrm{Fe}$ & 10 & 1.5 & 0.080 & 0.46 & 0.3 & $300^{c}$ \\
\hline Fecal coliform ${ }^{g}$ & 10 & 14 & 0 & 1.4 & 2.22 & $\begin{array}{l}1000^{e} \\
1.0^{f}\end{array}$ \\
\hline Gross alpha ${ }^{h, i}$ & 10 & 52 & 0.011 & 7.8 & 0.29 & $15 \mathrm{pCi} / \mathrm{L}^{\mathrm{f}}$ \\
\hline Gross beta $^{h, j}$ & 10 & 220 & .30 & 52 & 1.4 & $4 \mathrm{mrem} / \mathrm{yr}^{\mathrm{f}}$ \\
\hline $\mathrm{Hg}$ & 10 & $<0.0001$ & $<0.0001$ & $<0.0001$ & 0 & $0.002^{b}$ \\
\hline Lindane & 10 & $<0.002$ & $<0.002$ & $<0.0020$ & 0 & $0.004^{b}$ \\
\hline Methoxychlor & 10 & $<0.01$ & $<0.01$ & $<0.01$ & 0 & $0.1^{\mathrm{b}}$ \\
\hline $\mathrm{Mn}$ & 10 & 4.0 & 0.07 & 1.3 & 1.0 & $50^{c}$ \\
\hline $\mathrm{Na}$ & 10 & 30 & 14 & 20 & 3.0 & \\
\hline Nok & 10 & $<5.0$ & $<5.0$ & $<5.0$ & 0 & $10^{f}$ \\
\hline $\mathrm{Pb}$ & 10 & 0.040 & $<0.02$ & $<0.022$ & 0.028 & $0.05^{b}$ \\
\hline $\mathrm{pH}^{1}$ & 126 & 8.2 & 7.2 & 7.5 & 0.05 & $6-9^{e}$ \\
\hline Phenols & 10 & 0.002 & $<0.001$ & $<0.0013$ & 0.0 & \\
\hline $\mathrm{Ra}(\text { total })^{\mathrm{h}, \mathrm{m}}$ & 10 & 0.037 & $<0.011$ & $<0.015$ & 0.0002 & $5 \mathrm{pCi} / \mathrm{L}^{\mathrm{f}}$ \\
\hline Se & 10 & $<0.005$ & $<0.005$ & $<0.005$ & 0.0 & $0.01^{b}$ \\
\hline $\mathrm{SO}_{4}$ & 10 & 100 & 19 & 52 & 21 & $250^{c}$ \\
\hline Specific conductance ${ }^{n}$ & 70 & 0.49 & 0.03 & 0.23 & 0.02 & \\
\hline Temperature $^{\circ}$ & 70 & 22 & 8.8 & 16 & 0.78 & \\
\hline Total organic carbon & 40 & 3.8 & 1.1 & 2.4 & 0.22 & \\
\hline Total organic halides & 40 & 0.07 & 0.01 & 0.03 & 0.0 & \\
\hline Toxaphene & 10 & $<0.005$ & $<0.005$ & $<0.005$ & 0 & $0.005^{t}$ \\
\hline
\end{tabular}

95\% confidence coefficient (CC) about the average.

${ }^{b}$ Code of Federal Regulations, Part 40, Subpart 141. National Primary Drinking Water Maximum Concentration Limits (MCL).

'Tennessee Department of Health and Environment (TDHE). State Secondary Drinking Water Regulation, Chapter 1200-5-1, as ammended November 10, 1988. 
Attachment A, CERCLA Specific Comme Response 94

${ }^{\mathrm{d}} \mathrm{MCL}$ listed is that for total chromium, but is based on the toxicity value for chromium VI

'TDHE. State Water Quality Standard MCL. Chapter 1200-4-3.

f TDHE, State Primary Drinking Water MCL, Chapter 1200-5-1, as amended November 10, 1988.

BUnits are colonies per 100 milliliters.

hUnits are becquerels per liter.

includes ${ }^{226} \mathrm{Ra}$ but excludes radon and uranium.

${ }^{k}$ Value is that for nitrate as nitrogen. MCL for nitrate is applicable to both community water systems and non-community water systems.

jChapter 122-5-1.06 of the rules of the TDHE specifies that if two or more radionuclides are present, the sum of their annual dose equivalent to the total body or to any organ shall not exceed $4 \mathrm{mrem} / \mathrm{year}$. Guidance on calculation of average annual concentrations assumed to produce a total body dose of 4 mrem/year is given.

'Value in $\mathrm{pH}$ units.

${ }^{m}$ Combined ${ }^{226} \mathrm{Ra}$ and ${ }^{228} \mathrm{Ra}$.

nUnits are micromhos per centimeter.

'Units are degrees Celsius.

Source: T. W. Oakes et al., Environmental Surveillance of the U.S. Department of Energy Oak Ridge Reservation and Surrounding Environs 1986, ES/ESH-1/V1, Oak Ridge National Laboratory. 
Attachm',nt A, CERCLA Specific Comment Response 94

Radionuclide, Fe, and Mn concentrations in stream gravels within WAG I

Sample Month Year North East $\quad \mathrm{Mn}^{\mathrm{a}} \quad \mathrm{Fe}^{\mathrm{a}} \quad{ }^{60} \mathrm{Co}^{\mathrm{b}} \quad{ }^{137} \mathrm{Cs}^{\mathrm{b}} \quad{ }^{90} \mathrm{Sr}^{\mathrm{b}}$

\begin{tabular}{|c|c|c|c|c|c|c|c|c|c|}
\hline \multicolumn{10}{|c|}{ White Oak Creek } \\
\hline 1 & 10 & 78 & 21360 & 32545 & $\cdot 1220$ & 770 & 0.0 & 0.0 & 0.9 \\
\hline 2 & 10 & 78 & 21365 & 32435 & 910 & 800 & 0.0 & 0.0 & 0.2 \\
\hline 3 & 10 & 78 & 21365 & 32335 & 2040 & 1010 & 0.0 & 0.3 & 0.1 \\
\hline 4 & 10 & 78 & 21365 & 32235 & 1910 & 995 & 0.0 & 0.0 & 0.4 \\
\hline 5 & 10 & 78 & 21370 & 32135 & 770 & 665 & 0.0 & 1.7 & 0.1 \\
\hline 6 & 10 & 78 & 21370 & 32020 & 600 & 565 & 0.0 & 0.0 & 0.4 \\
\hline 7 & 10 & 78 & 21370 & 31910 & 750 & 540 & 0.0 & 0.0 & 0.0 \\
\hline 8 & 10 & 78 & 21370 & 31795 & 780 & 635 & 0.0 & 0.0 & 0.5 \\
\hline 9 & 10 & 78 & 21345 & 31685 & 1130 & 945 & 1.3 & 13.0 & 3.3 \\
\hline 10 & 10 & 78 & 21285 & 31590 & 570 & 560 & 0.0 & 8.5 & 0.5 \\
\hline 11 & 10 & 78 & 21225 & 31495 & 1350 & 745 & 1.6 & 10.8 & 1.0 \\
\hline 12 & 10 & 78 & 21165 & 31365 & 780 & 460 & 0.0 & 6.2 & 1.2 \\
\hline 13 & 10 & 78 & 21160 & 31285 & 920 & 690 & 1.0 & 4.4 & 1.9 \\
\hline 14 & 10 & 78 & 21170 & 31270 & 422 & 1120 & 0.0 & 15.4 & 22.4 \\
\hline 15 & 10 & 78 & 21155 & 31165 & 1300 & 775 & 3.1 & 7.6 & 2.8 \\
\hline 16 & 10 & 78 & 21155 & 31065 & 630 & 720 & 0.0 & 52.4 & 3.2 \\
\hline 17 & 10 & 78 & 21160 & 30945 & 1030 & 890 & 13.3 & 558.0 & 5.3 \\
\hline 18 & 10 & 78 & 21175 & 30960 & 476 & 440 & 25.9 & 72.5 & 18.2 \\
\hline 19 & 10 & 78 & 21165 & 30845 & 465 & 610 & 17.6 & 2300.0 & 4.5 \\
\hline 20 & 10 & 78 & 21175 & 30835 & 128 & 355 & 370.0 & 1190.0 & 2.5 \\
\hline 21 & 10 & 78 & 21140 & 30710 & 595 & 790 & 116.3 & 1690.0 & 9.7 \\
\hline 22 & 10 & 78 & 21135 & 30630 & 389 & 755 & 127.0 & 1200.0 & 9.3 \\
\hline 23 & 10 & 78 & 21125 & 30530 & 1050 & 970 & 147.0 & 1470.0 & 6.8 \\
\hline 24 & 10 & 78 & 21115 & 30415 & 605 & 895 & 112.0 & 3470.0 & 9.7 \\
\hline 25 & 10 & 78 & 21035 & 30345 & 695 & 615 & 94.0 & 2370.0 & 4.5 \\
\hline 26 & 10 & 78 & 20945 & 30260 & 585 & 535 & 98.0 & 2620.0 & 3.9 \\
\hline 27 & 10 & 78 & 20865 & 30190 & 685 & 790 & 110.0 & 1500.0 & 8.9 \\
\hline 28 & 10 & 78 & 20785 & 30120 & 420 & 810 & 186.0 & 4720.0 & 8.4 \\
\hline 29 & 10 & 78 & 20705 & 30045 & 420 & 765 & 164.0 & 6280.0 & 6.5 \\
\hline 30 & 10 & 78 & 20725 & 30045 & 605 & 1000 & 130.0 & 3030.0 & 18.2 \\
\hline 31 & 10 & 78 & 20620 & 29965 & 485 & 1000 & 93.4 & 3110.0 & 6.7 \\
\hline 32 & 10 & 78 & 20535 & 29890 & 380 & 1150 & 195.0 & 23200.0 & 17.2 \\
\hline 33 & 10 & 78 & 20460 & 29810 & 465 & 1080 & 190.0 & 6990.0 & 12.6 \\
\hline
\end{tabular}

First Creek

$\begin{array}{llcccccccc}400 & 2 & 79 & 21135 & 29625 & 775 & 1610 & 0.0 & 8.4 & 1.5 \\ 401 & 2 & 79 & 21250 & 29620 & 545 & 1310 & 0.0 & 5.0 & 2.2 \\ 402 & 2 & 79 & 21380 & 29620 & 805 & 815 & 0.0 & 0.0 & 1.8 \\ 403 & 2 & 79 & 21505 & 29620 & 670 & 1110 & 0.0 & 0.7 & 1.1 \\ 404 & 2 & 79 & 21630 & 29625 & 860 & 890 & 0.0 & 0.4 & 1.6\end{array}$


Attachment A, CERCLA Specific Comment Response 94

(continued)

\begin{tabular}{lccccccccc}
\hline Sample & Month & Year & North & East & $\mathrm{Mn}^{\mathrm{a}}$ & $\mathrm{Fe}^{\mathrm{a}}$ & ${ }^{60} \mathrm{Co}^{\mathrm{b}}$ & ${ }^{137} \mathrm{Cs}^{\mathrm{b}}$ & ${ }^{90} \mathrm{Sr}^{\mathrm{b}}$ \\
\hline 405 & 2 & 79 & 21785 & 29620 & 430 & 670 & 0.0 & 1.3 & 1.1 \\
406 & 2 & 79 & 21915 & 29620 & 1370 & 1160 & 0.0 & 0.0 & 2.0 \\
407 & 2 & 79 & 22090 & 29620 & 600 & 1450 & 0.0 & 0.4 & 0.0 \\
408 & 2 & 79 & 22255 & 29630 & 540 & 690 & 0.0 & 1.4 & 1.0 \\
409 & 2 & 79 & 22420 & 29620 & 640 & 1410 & 0.0 & 0.9 & 1.6 \\
410 & 2 & 79 & 22575 & 29620 & 300 & 1100 & 0.0 & 0.2 & 1.2 \\
411 & 2 & 79 & 22735 & 29630 & 1380 & 1580 & 0.0 & 1.1 & 1.3 \\
412 & 2 & 79 & 22880 & 29555 & 1060 & 910 & 0.0 & 0.9 & 1.2 \\
383 & 2 & 79 & 20805 & 29565 & 1030 & 1350 & 0.0 & 132.0 & 2.4 \\
384 & 2 & 79 & 20915 & 29570 & 795 & 1020 & 0.0 & 86.7 & 1.9 \\
385 & 2 & 79 & 21020 & 29600 & 675 & 1270 & 0.0 & 272.0 & 5.5 \\
& 2 & & & & & & & & \\
\hline
\end{tabular}

${ }^{a} \mathrm{Mn}$ and $\mathrm{Fe}$ units are $\mu \mathrm{g} / \mathrm{g}$ of gravel.

${ }^{b}$ Radioactivity units are in disintegrations per minute per gram of gravel $(B q=60 \mathrm{dpm})$.

${ }^{c}$ WOC samples upstream of its junction with Northwest Tributary/First Creek.

dSource Cerling and Spalding (1981) 


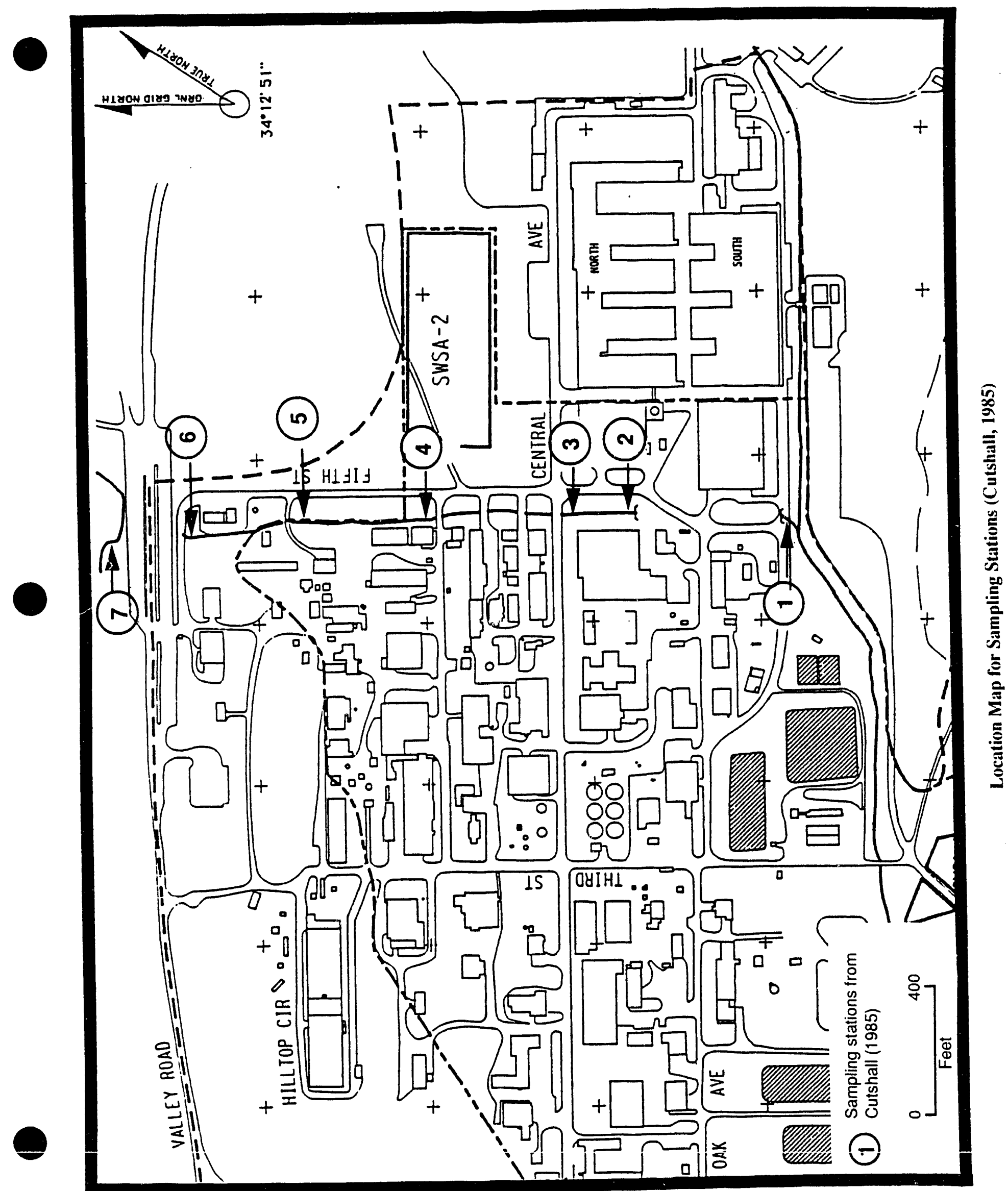


Attachment A, CERCLA Specific Comment Response 98 .

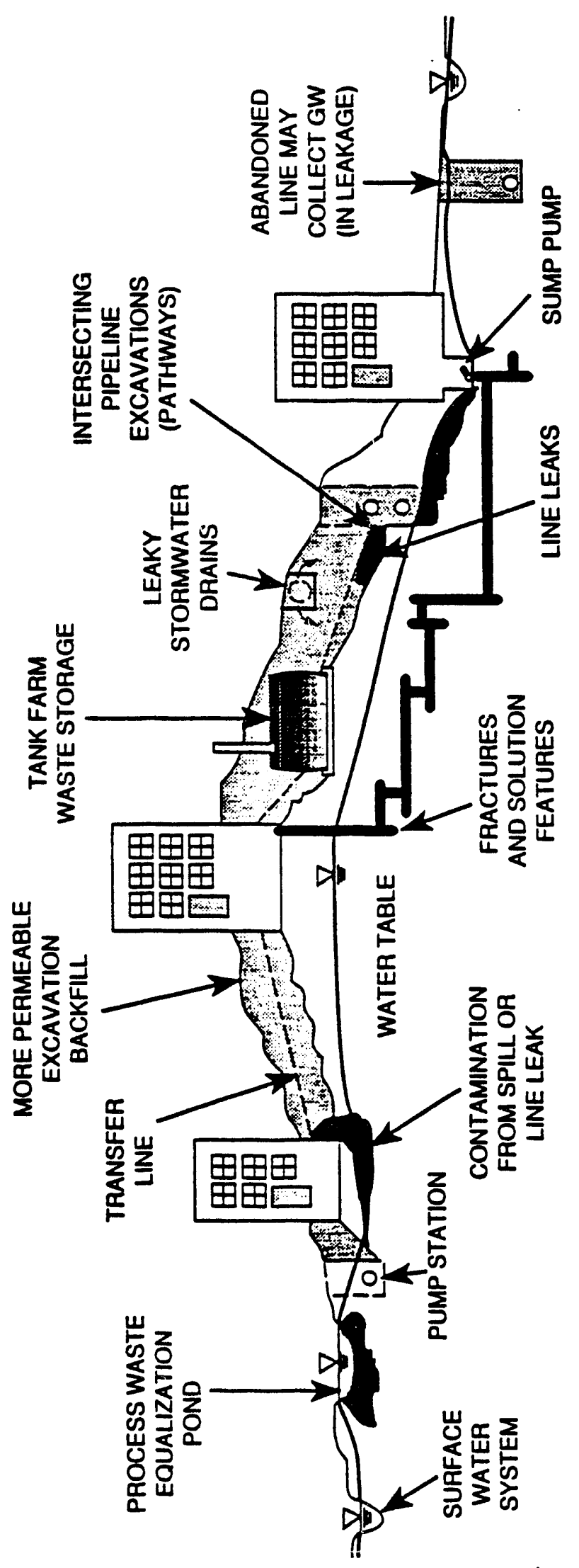

है 


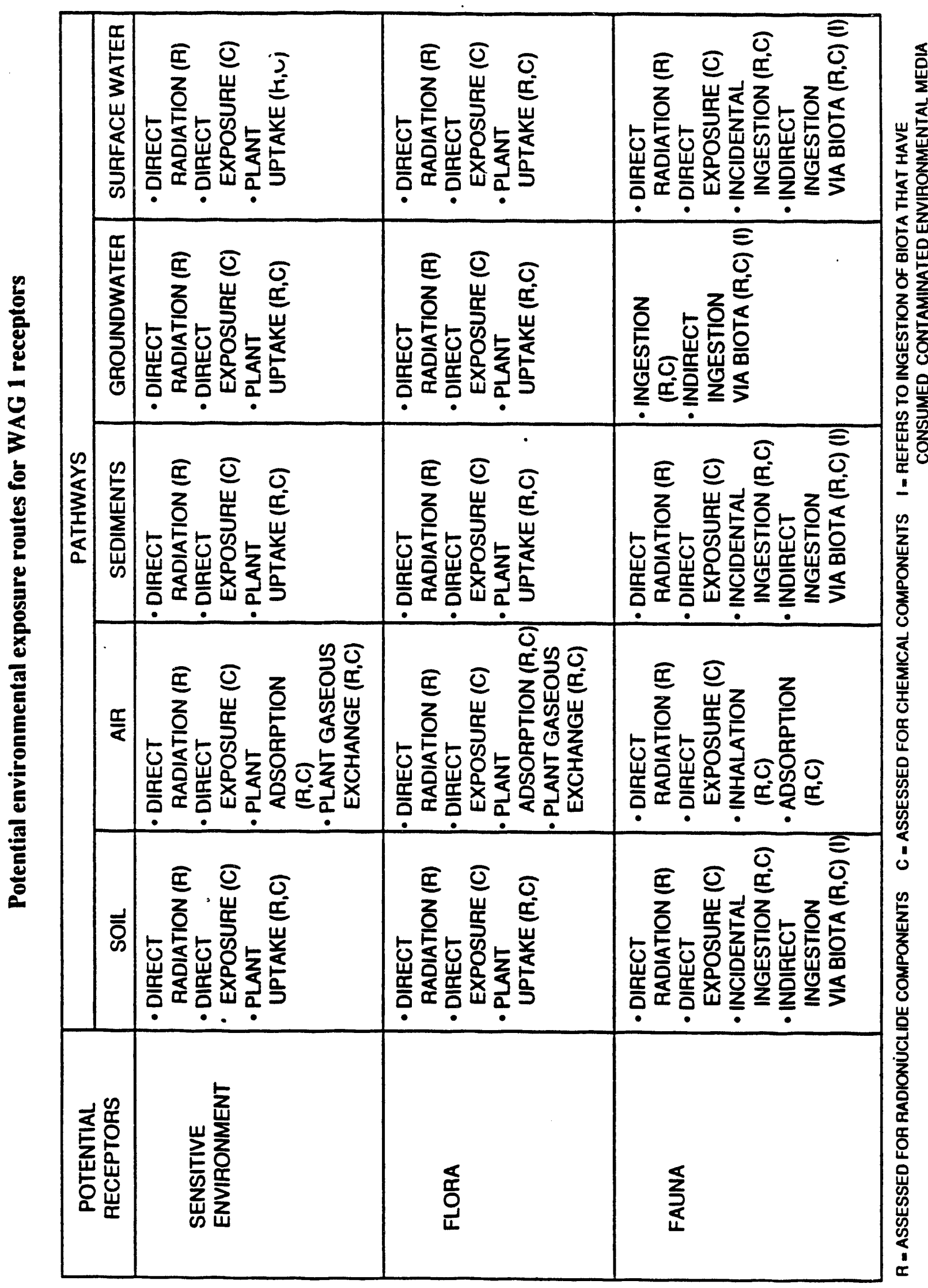




\section{A8. REFERENCE SAMPLING}

\section{A8.1 OBJECTIVES}

The objective for reference (background) sampling is to determine the levels of chemical and radiological constituents in the various environmental media at ORNL that have not been influenced by ORNL operations or waste disposal activities.

The Main Plant, WAG 1 , is underlain by limestone, siltstone, and shales of the Chickamauga Group. These rocks weather to clays containing variable amounts of chert. These clays consist primarily of a mixture of the minerals kaolinite and illite, but significant smectite content may be found locally.

\section{A8.2 LOCATION, FREQUENCY, AND ANALYSES}

\section{A8.2.1 Surface Water}

To provide an estimate of surface stream water quality unaffected by inputs from the waste units, samples will be collected from three locations within Bethel Valley. The locations are at the headwaters of the major streams that drain the ORNL plant area.

Surface water reference samples will be collected at locations indicated in Fig. A8.1 on First Creek, Fifth Creek, and WOC. One surface water sample will be collected from each creek during each of two events (one storm flow and one base flow) timed to occur during the high and the low of the regional stream hydrograph. Additional samples will be collected on the same schedule dictated for the WAG 1 surface water sampling effort.

\section{A8.2.2 Sediments}

Stream sediments will be collected at the locations indicated in Fig. A8.1 on a onetime basis. Samples will be collected from the stream channel according to Method Number ESP-303-5, "Subsurface Soil Sampling with Shelby Tubes" (MMES, 1988a), to a depth of 18 in. Samples will be composited prior to placement in sample containers except for the volatile organics, which will be collected directly from the shelby tube. Each sediment sample will be analyzed for TCL/TAL analytes, gross alpha, gross beta, and appropriate radionuclides.

In addition to the soil borings, data from the remote soil sampling locations that serve as part of the annual environmental surveillance program will be used to provide background radiation levels. 


\section{A8.2.3 Groundwater}

To evaluate reference conditions, groundwater samples from both the soil and bedrock flow systems, where both flow systems exist, should be collected. Existing wells capable of supplying baseline groundwater chemistry samples will be used.

An estimated 15 or 16 monitoring wells are needed to provide a reasonable estimate (95 percent confidence) of the natural variability of water quality parameters for the groundwater system around ORNL in Bethel Valley (Moore, 1989). Seven wells are to be installed to the west and west of the ORNL facilities in Bethel Valley (Fig. A8.1). Four wells will be located in WAG 17 (Fig. A8.2), three wells will be located in the WAG 3 locale (Fig. A8.3) and, three wells will be located within the WAG 1 Main Plant Area (Fig. A8.4). The six wells in WAGs 1 and 3 have already been installed. The four upgradient wells in WAG 17 need to be installed, as do the seven wells labeled BV01 through BV07 shown in Fig. A8.1. The three wells in WAG 1 have been sampled by ORNL for two quarters, but the three wells in WAG 3 have yet to be developed. Three additional prospective upgradient wells in WAG 1 were rejected because they showed elevated concentrations of various contaminants (i.,e., Well 815 showed the presence of cyanides; Well 816 had the only detectable concentration of beryllium, as well as elevated radium; Well 828 had the highest aluminum concentrations, as well as a high nitrate concentration).

Wells will be installed to conform to RCRA specifications using 2-in. stainless steel screen and riser pipe. Screen will be set at depths to sample the water table in the uppermost saturated zone. Samples will be collected semiannually to correspond to seasonal high and low water level periods as noted from local well hydrographs. Two distinct sampling events will be undertaken during each period. This schedule will enable the program to meet the intent of 40 CFR 264.98 (d) and 264.98 (f). Samples from the high and low water table sampling events will be analyzed for TCL/TAL constituents, gross alpha and gross beta, and an appropriate suite of radionuclides (including uranium isotopes. thorium isotopes, radium isotopes, potassium-40, tritium, and carbon-14).

Groundwater well installation, development, and sampling will be performed in accordance with Method Numbers ESP-600, "Groundwater Sampling Procedures: Well Installation, Development and Abandonment," and appropriate ESP-302, "Groundwater Sampling Procedures" (MMES, 1988a).

\section{A8.2.4 Soils}

To provide an estimate of reference concentrations of constituents unaffected by ORNL operations, reference samples will be collected from nine locations in Bethel Valley.

Reference soil samples in Bethel Valley will be collected during installation of nine groundwater monitoring wells: four in WAG 17, and the seven locations east and west of the ORNL facilities, numbered BV01 through BV07 (Fig. A8.1). 
Attachment A, CERCLA Specific Comment Response 143

Soil samples are routinely collected from remote stations as part of the environmental surveillance program at ORNL. Data from these sites will be used for radiological background for the environmental evaluation at WAG 1.

\section{A8.2.5 Vegetation}

As part of the environmental surveillance program at ORNL, vegetation samples are collected annually from remote monitoring stations. These data will be used as background for the environmental evaluation at WAG 1.

\section{A8.3 QUALITY CONTROL}

Appropriate QC samples (including duplicates, trip blanks, and equipment blanks) will be collected and submitted during each sampling event to conform with Method Number ESP-400, "Field Quality Control" (MMES, 1988a). Due to the importance of reference data, all analyses will receive Level III Contract Laboratory Program (CLP) data validation, which will include complete CLP data validation packages. 


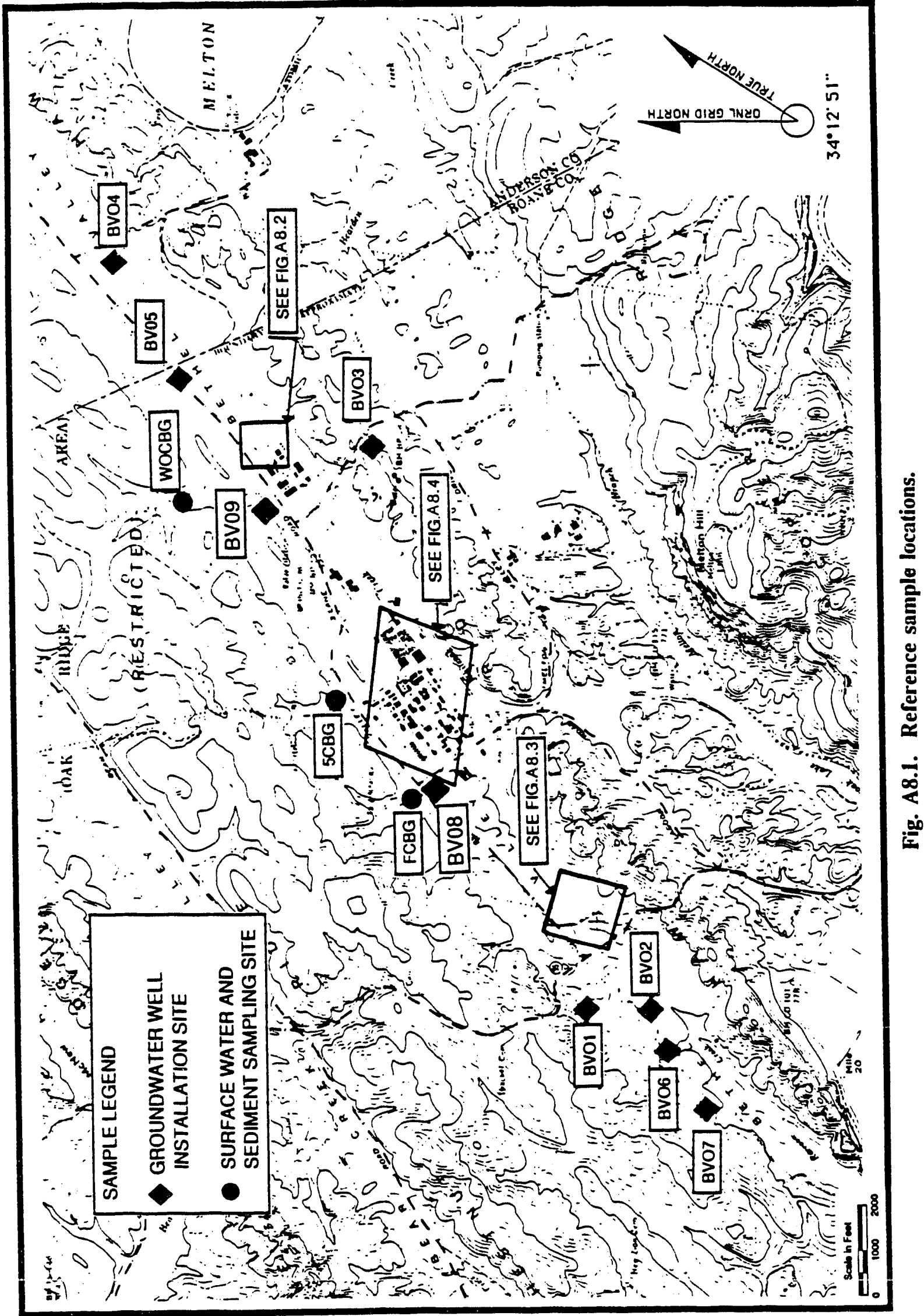




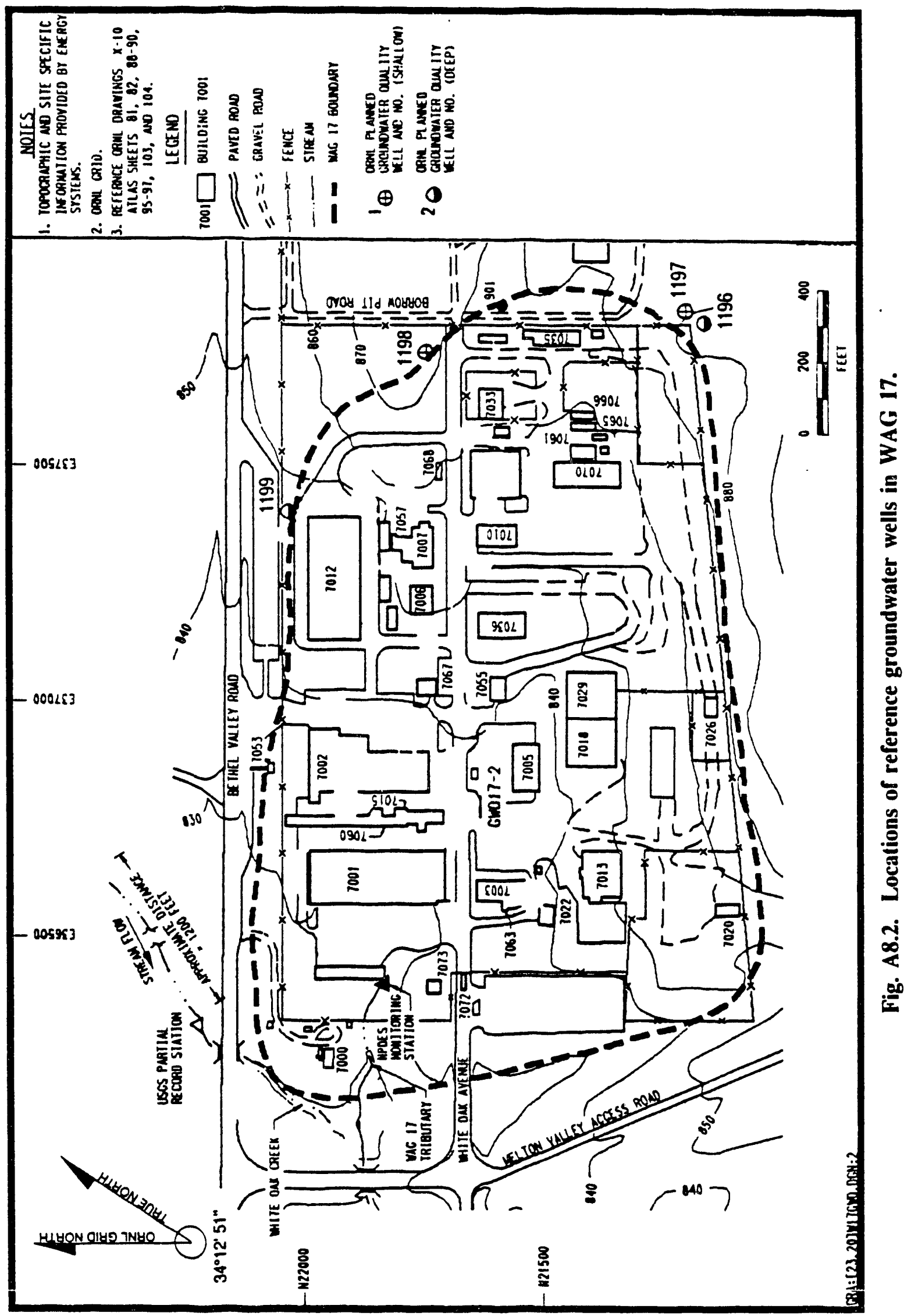


Attachment A, CERCLA Specific Comment Response 143

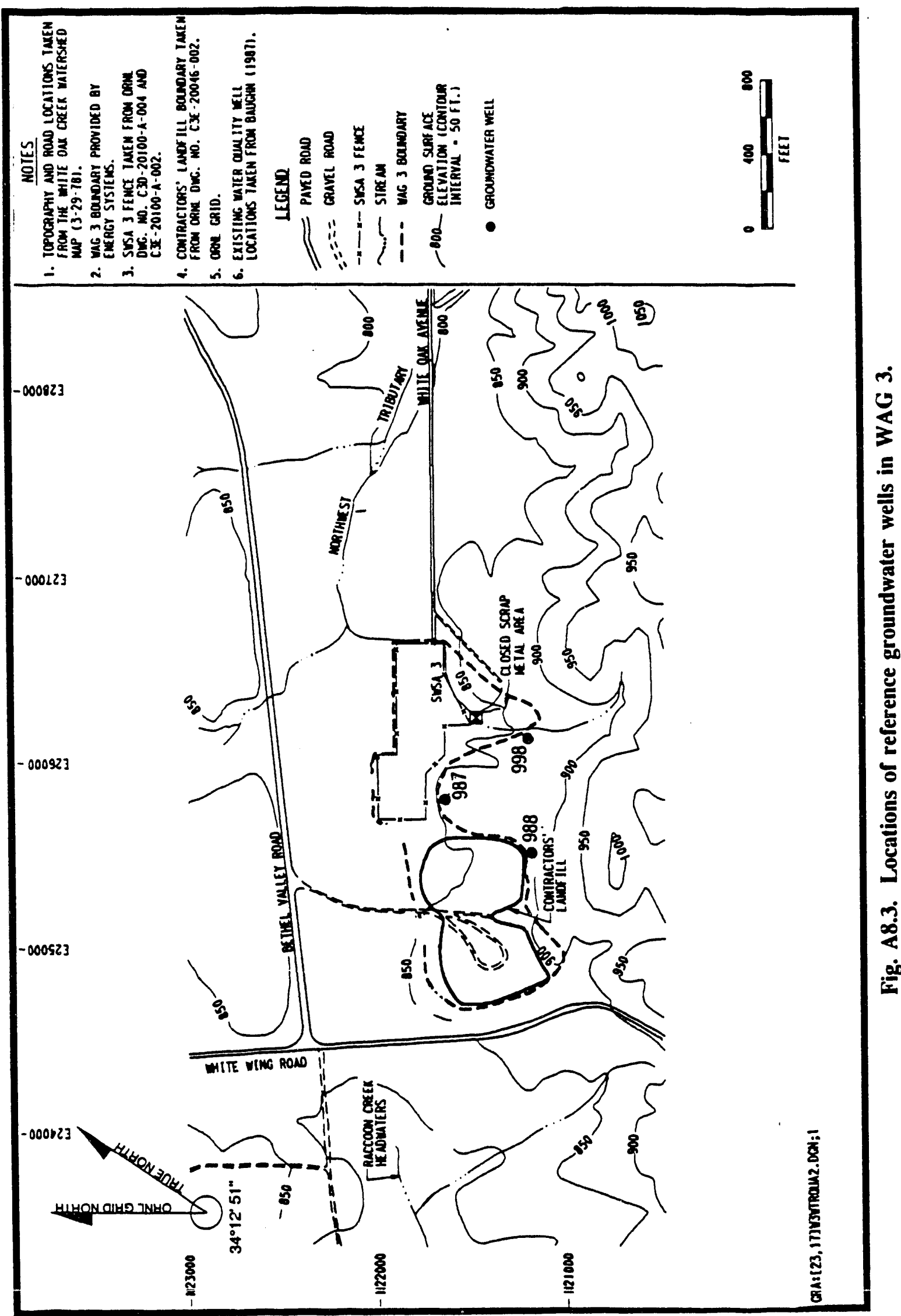

A-43 
Attachment A, CERCLA Specific Comment Response 143

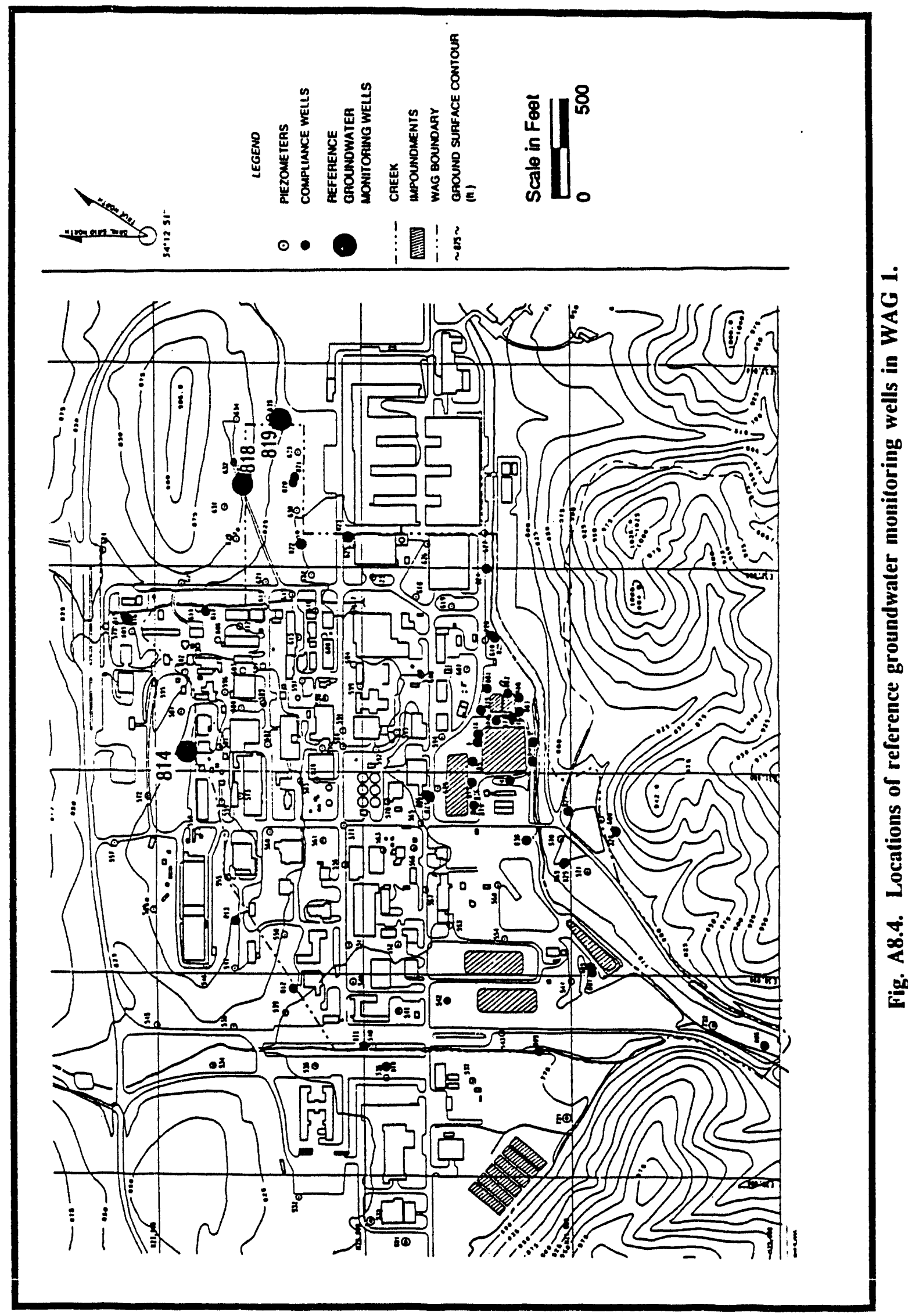


Attachment B, Part A - RCRA General Comments Response 6

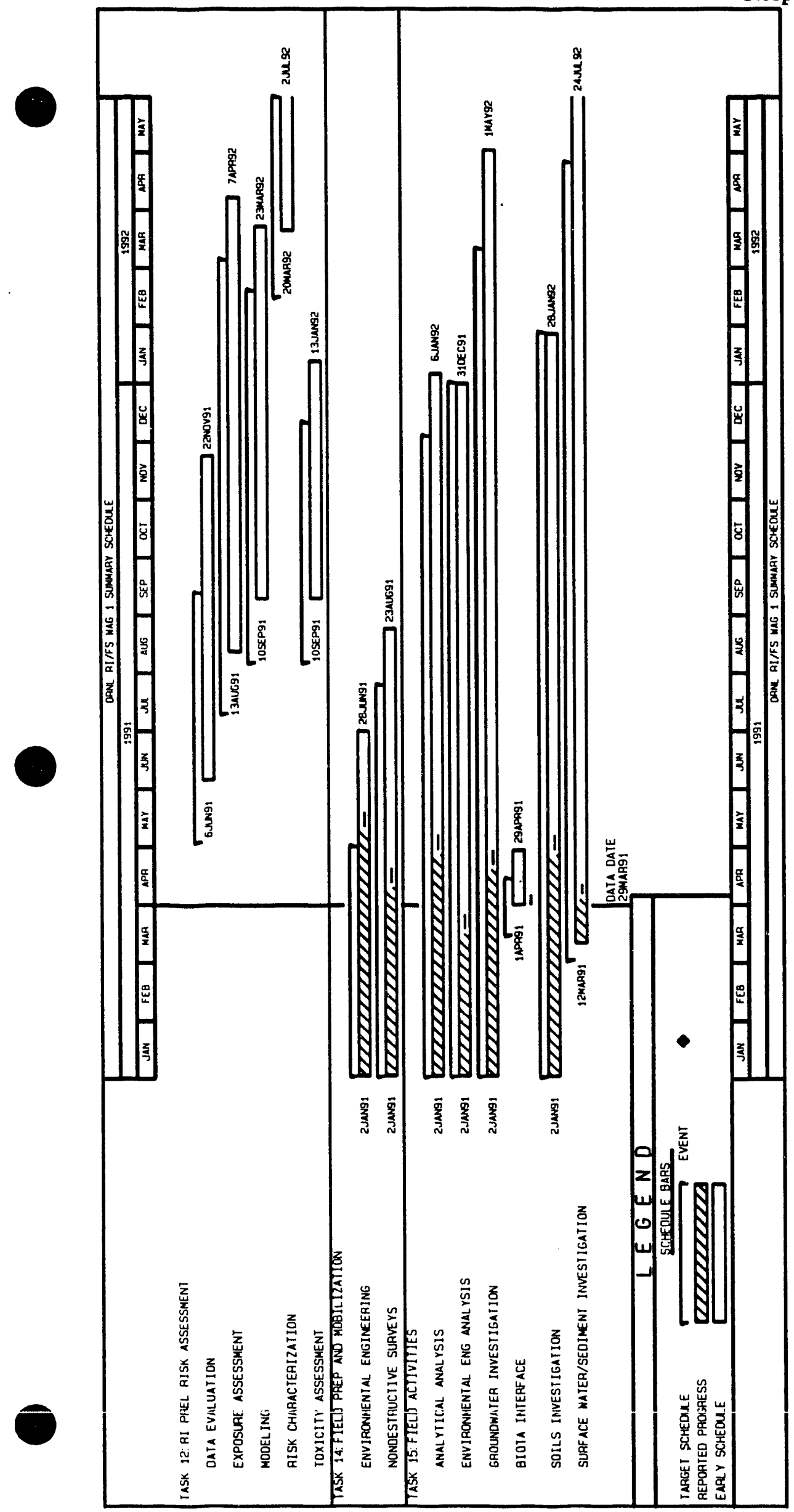


Attachment B, Part A - RCRA Specific Comments

Response 20

1986 PIEZOMETER SCOPING SURVEY

\begin{tabular}{|c|c|c|c|c|c|c|c|}
\hline 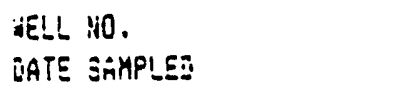 & & $\begin{array}{c}542 \\
0 i-15-j j\end{array}$ & $\begin{array}{c}944 \\
14-15-36\end{array}$ & $\begin{array}{c}548 \\
07-14-30\end{array}$ & $\begin{array}{c}550 \\
07-14-a b\end{array}$ & $\begin{array}{c}354 \\
i-i 4-86\end{array}$ & $\begin{array}{c}560 \\
4-22-86\end{array}$ \\
\hline PARAMETER & UNITS & & & & & & \\
\hline SIC̈́rRBOHATE & ME/L & 245 & $\$ 96$ & 305 & 245 & 270 & 215 \\
\hline CHLORIDE & UG/RL & $2 !$ & 16 & 99 & 9 & 15 & 8 \\
\hline FLUORISE & US/ML & 41 & 1 & 11 & 11 & il & 1 \\
\hline MLTRATE & UG/AL & 15 & $<5$ & 15 & $b$ & & $<5$ \\
\hline SULFATE & UG/ML & $2 b$ & 610 & 32 & 93 & 22 & 18 \\
\hline ALUMINUM & $U E / \mathrm{KL}$ & 6 & 0 & $1)$ & 5 & $<0$ & 1 \\
\hline BAR!L! & UE/ML & 1) & 0 & 0 & 0 & 0 & 0 \\
\hline CALCIUA & UG/ML & 200 & $6 j 0$ & 130 & 460 & 100 & 24 \\
\hline IROH & U6/ML & 3 & 1 & 0 & 3 & 1) & 0 \\
\hline MAGHESIUM & $106 / \mathrm{mL}$ & 11 & 64 & 28 & 14 & 8 & 5 \\
\hline MANEANESE & UG/AL & 2 & 1 & 0 & 1 & 0 & 0 \\
\hline sediun & UG/RL & $! 5$ & 260 & 14 & 8 & $<25$ & 5 \\
\hline S!L!COH & US/AL & 11 & 7 & $b$ & 7 & 4 & $?$ \\
\hline STRONTIUA & UG/AL & 0 & 2 & 0 & 1 & 0 & D) \\
\hline ZINC & UG/AL & 0 & 1) & 1) & 0 & & $1)$ \\
\hline GROSE ILFHA & $30 / L$ & 0.424 & 0.562 & 0.244 & 0.517 & -9.003 & 0.0246 \\
\hline GROSS SETA & $B Q / L$ & $1.528^{\prime}$ & 1.117 & 0.58 & 1.585 & 10.136 & 0.1265 \\
\hline iñlTiljn & $30 / L$ & 28.257 & 0.165 & !5t.665 & $105.808^{\circ}$ & 1.307 & 10.1728 \\
\hline & UE/ML & I & 4 & 2 & 2 & 3 & 2 \\
\hline CAGROON TETRACHLCRIDE & U6/L & $\langle 3$ & $<3$ & $<3$ & $\langle z$ & $<3$ & 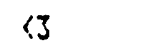 \\
\hline CHLOKOFORH & U6/L & 6 & $<2$ & $<2$ & 2 & (2 & 14 \\
\hline DICHLQROBROMOMETHAHE & $106 / \mathrm{L}$ & $<2$ & $<2$ & $<2$ & $\lfloor 2$ & $<2$ & $<2$ \\
\hline 1,!-D!CHLOROETHENE & $U 6 / L$ & $<3$ & (3 & $\langle J$ & $<3$ & 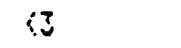 & $<3$ \\
\hline TETRACHLORUETHENE & U6/L & 28 & $<4$ & $<4$ & $<4$ & $<4$ & $<4$ \\
\hline TOLUEYY & $166 / L$ & $<5$ & $<5$ & $(5$ & is & EST $<5.000$ & EST $<S$ \\
\hline METHYLENE CHLORIDE & $46 / L$ & i3 & 13 & $\langle 3$ & $<3$ & $<3$ & $<3$ \\
\hline TRANS-i, 2 -DICHLORCETHEME & $46 / L$ & 2 & $<2$ & 10 & $<2$ & 32 & 12 \\
\hline TRICHLOROETHEME & U6/L & $<2$ & 42 & 40 & 4 & $<2$ & i2 \\
\hline ACETDNE & UG/L & NT & NT & HT & MT & NT & NT \\
\hline CDISELLFIDE & UE $\mathrm{iL}$ & NT & NT & NT & NT & MT & NT \\
\hline $9 H$ & STO & 7.8 & 7.3 & 7.4 & 3.3 & 7.6 & 7.1 \\
\hline TEMPERATURE & $c$ & 25.1 & 15.4 & 25.4 & $20 . j$ & 15.6 & 19.4 \\
\hline CONDUCTIYITY & UHHOS & 240 & 1630 & 500 & 320 & 180 & 40 \\
\hline
\end{tabular}




\begin{tabular}{|c|c|c|c|c|c|c|c|}
\hline $\begin{array}{l}\text { WELLL MO. } \\
\text { JATE SAMFLED }\end{array}$ & & $\begin{array}{c}612 \\
b-1) 4-36\end{array}$ & $\begin{array}{c}613 \\
4-22-36\end{array}$ & $\begin{array}{l}514 \\
5-145-36\end{array}$ & $\begin{array}{c}515 \\
7-09-96\end{array}$ & $\begin{array}{l}5 ! 6 \\
5-29-96\end{array}$ & $\begin{array}{c}317 \\
7-199-36\end{array}$ \\
\hline PAARRAMETER & UNITS & & & & & & \\
\hline BICARBOMATE & HG/L & 244 & 157 & 195 & 2.56 & 191 & 263 \\
\hline CHLQRIDE & U6/FL & 3 & 21 & 23 & 130 & 5 & 32 \\
\hline FLUORIDE & UG/ML & $\ll 1$ & $<1$ & $\longleftrightarrow 1$ & 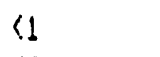 & $<1$ & $<1$ \\
\hline NITRATE & U6/AL & $<5$ & 12 & $\langle 5$ & 15 & $\langle 5$ & $<5$ \\
\hline SULFATE & UG/Ah & 38 & 85 & 130 & 55 & 120 & 37 \\
\hline ALUHINUM & UE/ML & 2 & 3 & 1 & 3 & 2 & 0 \\
\hline GARIUK & UG/ML & 0 & 0 & 0 & 0 & 0 & 1) \\
\hline CALCIUH & U6/ML & 55 & 600 & 110 & 440 & 260 & 100 \\
\hline IRON & UG/AL & 0 & 0 & 2 & 5 & 1 & 0 \\
\hline MAGNESIUH & UG/AL & 8 & 13 & 15 & 19 & 13 & $2 !$ \\
\hline MANGANESE & UG/FL & 1 & 5 & 1 & 1 & 1 & 0 \\
\hline soorun & UE/HL & 11 & 29 & 20 & NT & 20 & 16 \\
\hline SILICON & $U G / \mathrm{ML}$ & 8 & 6 & 7 & 8 & 8 & 7 \\
\hline STRCNTIUM & U6/ $/ \mathrm{HL}$ & 0 & 1 & 0 & 1 & 1 & 0 \\
\hline I!HC & $U 6 / \mathrm{ML}$ & $b$ & $<0$ & 1 & 1 & $<0$ & 1) \\
\hline GROSS ALPHA & $80 / \mathrm{L}$ & 1.88 & 0.206 & 0.074 & $<.020$ & 0.135 & 0.072 \\
\hline GROSS BETA & 9Q/L & 2.459 & 4.14 & $0.587^{\circ}$ & 9.425 & 0.887 & 4.802 \\
\hline TRITIUK & $B O / L$ & 531.532 & $1.4 j 1$ & 0.37 & 490.936 & 1.801 & 423.603 \\
\hline & UG/HL & 4 & 2 & 3 & 4 & 4 & 2 \\
\hline CARBON TETRACHLORIDE & U6/L & $<3$ & $<3$ & $<3$ & 3 & (3) & $\langle 3$ \\
\hline CHLDROFORH & $U G / L$ & $<2$ & $<2$ & $<2$ & $<2$ & 2 & $\langle 2$ \\
\hline DICHLGROBROMOHETHANE & UGil & i? & $\langle 2$ & $<2$ & $<2$ & 12 & 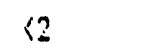 \\
\hline !, :-JICHLOKOETHENE & Ua/L & 88 & $<3$ & 《了 & $<3$ & $<3$ & i3 \\
\hline TETRACHLOROETHENE & $\sqrt{ } 6 / \mathrm{L}$ & jo & $<4$ & $<4$ & $<4$ & $\ll$ & $<4$ \\
\hline TOLUEHE & UE/L & EST 15 & EST 15 & EST $\angle S$ & ESTIS & EST & EST \\
\hline METHYYLENE CHLDRIDE & UE/L & 3 & 3 & 13 & 3 & 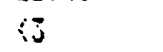 & 《3 \\
\hline TRANS-1, 2-DICHLOROETHENE & UG/L & $<2$ & 3 & 183 & $<2$ & $<2$ & $\langle 2$ \\
\hline TAICHLOROETHEME & U6/L & 19 & 3 & 7 & $<2$ & $\{2$ & $i \hat{z}$ \\
\hline ACETONE & UE/L & NT & NT & NT & NT & NT & NT \\
\hline CDISULFIDE & $106 / L$ & NT & NT & NT & NT & YTT & NT \\
\hline PH & STO & 8.3 & i.t & 7.8 & 7.5 & 8.1 & 7.2 \\
\hline TEAPSRATURE & c & 29.8 & 18.5 & 20.8 & 19.6 & 25.4 & 32.6 \\
\hline COMEUCTIVITY & UHHOS & 370 & 190 & 270 & 7.5 & 650 & 780 \\
\hline
\end{tabular}


WELL NO.

DATE SAMPIEŨ

\section{PARAXETEN̂}

BICARBONATE

CHLORIDE

FLUORIOE

NITRATE

SULFATE

ALUAINUH

BARIUH

CALCIUM

IRON

MAGNESIUM

MANGANESE

SOOIUN

SILICON

STRONT!liK

IiNC

GROSS ALPHA

SROSE BETA

TRITIUA

TOC

CARBON TETRACHLORIDE

CHLOROFORM

DICHLGROBROMOHETHANE

1.1-DICHLOROETHENE

TETRACHLOROETHENE

TOLUENE

METHYLENE CHLORIDE

TRANS-1, $\hat{\imath}$-I] ICHLOROETHEHE

TRICHLOROETHENE

ACETONE

CDISULFIDE

$\mathrm{PH}$

TEMPERATURE

COMDUCTIVITY

$$
\begin{array}{cccccc}
618 & 621 & 622 & 623 & 626 & 528 \\
94-30-36 & 4-30-86 & 5-05-86 & 5-15-96 & 5-29-86 & 7-198-36
\end{array}
$$

UNITS

\section{H6/L}

US/ML

UG/HL

UGIML

UG/ML

UG/HL

UG/ML

US/ML

UG/HL

UG/ML

UG/ML

UG/HL

UG/ML

U6/IIL

USS/ML

SO/L

$B Q / L$

$30 / L$

UG/ML

$U 6 / L$

UG/L

UE/L

$116 / L$

U6/L

UG/L

UG/L

UG/L

UG/L

UG/L

UG/L

STD

$c$

JMHOS

$\begin{array}{rrrr} & 396 & & 364 \\ & 39 & & 12 \\ 11 & & <1 & \\ & & <5 & \\ & 24 & & 65 \\ & 2 & 10 & \\ & 0 & & 0 \\ 510 & & 91 \\ 2 & & 0 \\ & & \\ & & & 43 \\ & 1 & & 0 \\ & 26 & & 39 \\ 10 & & 6 \\ 1 & & 2\end{array}$

$<0$

$$
0.222
$$

0.678

i). 255

$<0$

0.145

0.296

0.271

< 5

$<2$

$<2$

13

4

EST<5

$<2$
NT
NT

$\begin{array}{rrrr} & 7 & \langle 3 & \\ \langle 2 & & \langle 2 & \\ \langle 2 & & <2 & \\ \text { NT } & & \text { NT } & \\ \text { NT } & & \text { NT } & \\ & & & \\ & & & \\ & 18.7 & & 7.6 \\ & & 23.1 \\ & 410 & & 410\end{array}$

37

$<5$

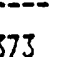

8

$-\cdots$

8

$$
41
$$

$<5$

$70<5$
2

$---$

\section{9}

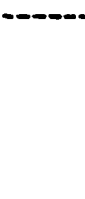

09

41

$1<$

$8<5$

410

2

260

2

14

?

28

28
7

1

0.144

0.623

$0.32 !$

?

i3

$<2$

$<2$

$<3$

4

EST $<5$

(3)

$<2$

$<2$

NT

NT

7.1

7.1

22.1
410

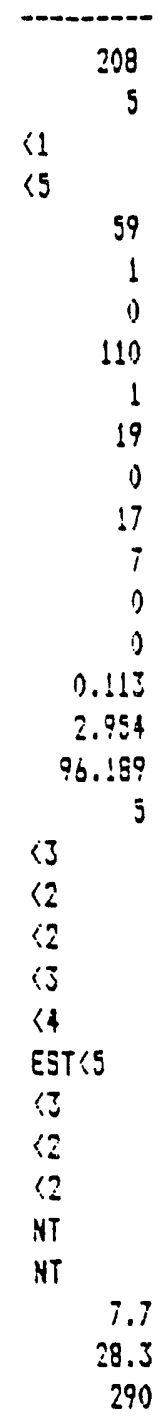


Attachment B, Part B - RCRA Specific Comments Response 7

f. The construction area between buildings 4508 and 4500 South;

g. The equipment storage area by building 3544 and

$h$. The fenced area surrounding buildings $3019,3001,3136$ and 3100 .

\subsection{QUALITY CONTROL}

\subsection{TRACK MAPS}

The survey track map is the map generated by the USRADS program. The track map shows the position of the SR's used and the path taken by the surveyor. Any areas above the selected threshold are highlighted on the track map. Track maps for the surveys performed are included in Appendix $C$.

\subsection{INSTRUMENTATION}

The instrumentation used for radiation surveys was returned each day prior to surveying to be source checked. Source checks were performed at the Bechtel FoF by Walt Keen using a depleted uranium source. Copies of the source check records are on file at the Bechtel FOF and the Chemrad office.

The instrumentation was calibrated on a three month frequency by the ORNI calibration facility. Calibration data is included in Appendix A.

The EM-31 was calibrated prior to use by the manufacturer and was field calibrated by the technicians using the operating manual.

\subsection{BACKGROUND}

Background readings were taken on a daily basis at the Bechtel FoF prior to surveying and were recorded in the field logs. The instruments were allowed to stabilize for approximately five minutes prior to recording the readings. The background readings are included in Appendix $B$.

\subsection{THRESHOLD CUTOFF DETERMINATION}

CPM: Selected ten grids around Hilltop circle where local area background is relatively low. The mean count rate for the grids was determined along with the standard deviation. The threshold cutoff ras then calculated at 17,832 by multiplying the background plus one standard deviation by three.

UR/HR: The threshold cutoff for dose was determined to be $86 \mathrm{UR} / \mathrm{HR}$ by taking the mean from the FOF background readings plus two standard deviations. 
Attachment B, Part B - RCRA Specific Comments Response 7

\subsection{APPENDICES}

Appendix A: Calibration Data

Appendix B: Background Readings

Appendix C: Summary Grids

Appendix D: EM-31 Data 
Attachment B, Part A - RCRA Specific Comments Response 20

\begin{tabular}{|c|c|c|c|c|c|c|c|}
\hline $\begin{array}{l}\text { HELL HO. } \\
\text { JATE SAHPLED }\end{array}$ & & $\begin{array}{r}561 \\
4-29\end{array}$ & $\begin{array}{l}96 j \\
7-9-96\end{array}$ & $\begin{array}{c}565 \\
5-27-36\end{array}$ & $\begin{array}{c}567 \\
i-1 b-d b\end{array}$ & $\begin{array}{c}568 \\
4-10-86\end{array}$ & $\begin{array}{c}570 \\
7-! 6-36\end{array}$ \\
\hline PARAMETER & UNITS & & & & & & \\
\hline DICARBOHATE & 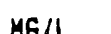 & 316 & 159 & 82 & 0 & 294 & 389 \\
\hline CHLOR!DE & UG/ML & $\begin{array}{r}610 \\
15\end{array}$ & 23 & 110 & 27 & $\begin{array}{r}479 \\
19\end{array}$ & $\begin{array}{r}289 \\
7\end{array}$ \\
\hline FLUORIDE & $116 / \mathrm{HL}$ & $\ll 1$ & 4 & $\langle 1$ & $<1$ & 11 & 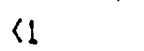 \\
\hline NITRATE & UG/ML & $<5$ & $<5$ & 15 & $<5$ & $<5$ & $<5$ \\
\hline SULFATE & UG/ML & 760 & 74 & 50 & 43 & 170 & 21 \\
\hline ALUMINUA & UG/FL & 0 & 4 & 2 & 16 & 0 & $!$ \\
\hline BARIUN & UG/ML & 0 & 0 & 0 & 1) & 0 & 0 \\
\hline CALCiUn & US/ML & 240 & 340 & 48 & 690 & 140 & 180 \\
\hline IRON & U6/ML & 0 & 1 & 1 & 4 & 0 & 2 \\
\hline MAGHESIUY & $U 6 / M L$ & 40 & 29 & 2 & 16 & 32 & 16 \\
\hline MANGANESE & $U S / \mathrm{HL}$ & 0 & 1 & 0 & 0 & 0 & 0 \\
\hline SOoluh & WGint & 57 & 19 & 81 & 26 & 25 & 7 \\
\hline SILICON & UG/ML & $b$ & 5 & 4 & 14 & $\cdot 7$ & 6 \\
\hline STRONTIUK & U6/ML & 1 & $!$ & 0 & & 0 & 0 \\
\hline ZIHC & U6/ML & 0 & 0 & $\langle 1)$ & 0 & $<0$ & 0 \\
\hline SROSS ALPHA & $B Q / L$ & 0.141 & 0.338 & 0.071 & 0.131 & 0.049 & $<.910$ \\
\hline SROSS SETA & $B Q / L$ & 2.281 & 2.9 & 0.379 &. .043 & 0.155 & 1.j?1 \\
\hline iRITIUH & $B Q / L$ & 0.222 & 125.046 & 0.173 &, 7.714 & 2.787 & 115.427 \\
\hline TOC & UG/ML & 4 & $j$ & 8 & 3 & 3 & 2 \\
\hline CARBON TETRACHLORIDE & $U G / L$ & $\langle 3$ & $\langle 3$ & $\langle 3$ & $<3$ & 《3 & $\langle 3$ \\
\hline CHLOROFORH & US/L & $<2$ & $<2$ & $<2$ & $<2$ & $\langle 2$ & 3 \\
\hline DICHLOROBROMOHETHANE & U6/L & $<2$ & $\langle 2$ & $\langle 2$ & $<2$ & $\lfloor 2$ & 12 \\
\hline 1.L-DICHLOROETHENE & $U S / L$ & $<3$ & $<3$ & i3 & $\langle 3$ & $\langle 3$ & $\langle 3$ \\
\hline TETRACHL OROETHENE & $U 6 / L$ & $<4$ & $<4$ & $\langle 4$ & $<4$ & $\langle 4$ & $<4$ \\
\hline TOLUF:AE & U6/L & 17 & EST<S & EST $<5$ & EST $<5$ & EST & EST $<5$ \\
\hline METHYYLENE CHLORIDE & UG/L & $\langle j$ & $\langle 3$ & $<3$ & $\langle ?$ & 13 & 17 \\
\hline TRHNS-1,2-OICHLOROETHENE & UE/L & $\langle 2$ & $<2$ & $<2$ & $\langle 2$ & 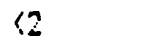 & 12 \\
\hline TRICHLOROETHENE & U6/L & $\langle 2$ & $<2$ & 7 & $<2$ & $\checkmark 2$ & $\langle\hat{\imath}$ \\
\hline ACETONE & U6/L & NT & NT & NT & NT & NT & NT \\
\hline CDISULFIDE & US/L & NT & NT & NT & NT & NT & NT \\
\hline PH & STO & 7.3 & 7.9 & 8.2 & 11.9 & 7.5 & 7.4 \\
\hline TEXPERATURE & C & 25 & 31.4 & 25.3 & 28.1 & 14.2 & 20.5 \\
\hline CONDUCTIVITY & UAHOS & 760 & 180 & 280 & 1930 & 370 & 210 \\
\hline
\end{tabular}


Attachment B, Part A - RCRA Specific Comments Response 20

WEIL NO.

CATE GAMPLED

PARAMETER

BICATBONATE

CHLORIDE

FLUOR!DE

MITRATE

SULFATE

ALUAINUM

BARIUM

CALCIUH

IRON

MAGNESIUH

MANGANESE

SODIUM

SILICON

STRONTIUKA

ZINC

GROSS ALPHA

GROSS BETA

TRITIUA

TOC

CARBON TETRACHLORIDE

C.HLOROFORH

DICHLOROBROMOHETHANE

1.1-DICHLOROETHENE

TETRACHLOROETHENE

TOLUENE

METHYLENE CHLORIDE

TRAMS-1,2-DICHLOROETHEME

TRICHLOROETHENE

ACETONE

CDISULFIDE

$\mathrm{PH}$

TEAPERATURE

CONDUCTIVITY

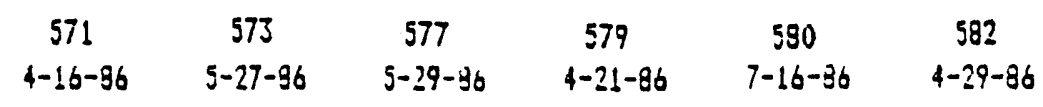

UNITS

146/L

UGiML

IJE/ML

U6/ HL

UG/ML

U6/ML

UG/ML

U6/ML

UG/ML

UG/FL

UG/ML

US/ML

UE/ML

UG/RL

U6/ ML

$B Q / L$

BQ/L

8Q/L

UE/ML

U6/

U6/L

Uล/L

U6/L

U $/ L$

U6/L

UGiL

UG/L

UG/L

U6/L

U6/L

STD

$C$

IMHOS
0.1246

4.4306

0.2057

0.046

(3)

12

$<2$

(3)

44

EST

$<3$

$<2$

(2)

NT

NT

7.5

12.2

390

\begin{tabular}{|c|c|c|}
\hline 388 & & 29 \\
\hline$b$ & & 35 \\
\hline 《1 & 《I & \\
\hline & & 4 \\
\hline $\begin{array}{r}110 \\
7\end{array}$ & & 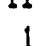 \\
\hline 0 & & 0 \\
\hline 340 & & 70 \\
\hline 12 & & 0 \\
\hline 19 & & 3 \\
\hline 2 & & 0 \\
\hline 56 & & 12 \\
\hline 10 & & 5 \\
\hline 1 & & 0 \\
\hline 0 & <0 & \\
\hline
\end{tabular}

$<0$

0.101

1.238

0.189

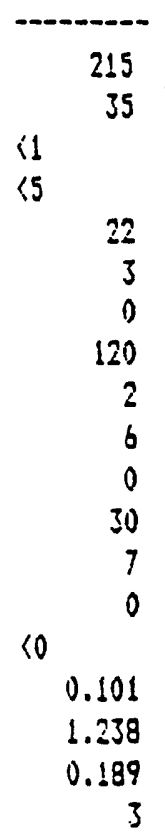

<3

42

$<2$

13

$<4$

EST $<5$

(3)

$<2$

$<2$

NT

NT
0.391

0.23

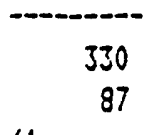

$<1$

$<5$

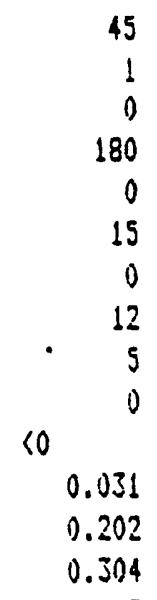

i3

$<2$

$<2$

i3

$\measuredangle 4$

EST $<5$

(3)

(2

$<2$

NT

NT

8.4

8.3

25.7

110
23.3

190

$\begin{array}{rrrr} & 289 & & 154 \\ & 4 & & 5 \\ 15 & & 11 & \\ & & <5 & \\ & 26 & & 41 \\ & 2 & <0 & \\ & 0 & & 0 \\ & 450 & & 78 \\ & 4 & & 1 \\ & 15 & & 7 \\ & 0 & & 1 \\ & 21 & & 12 \\ & 6 & & 2 \\ \text { HT } & & & 0 \\ & & & \\ & & & 1\end{array}$

$<0.472$

$14.966 \quad 18.9660$

$57.714 \quad 0.2301$

$\begin{array}{llll} & 4 & & 2 \\ <2 & & <3 & \\ <2 & & & 2\end{array}$

$<3$

$<4$

<3

44

EST $<5 \quad$ EST $<5$

33

$<2$

$(2$

NT

NT

7
16.7
660

$<2$

NT

NT

$\begin{array}{rr}7.8 & 7.7 \\ 19.9 & 2 b \\ 160 & 70\end{array}$




\begin{tabular}{|c|c|c|c|c|c|c|c|}
\hline $\begin{array}{l}\text { DELL NO. } \\
\text { JAIE BAMPLED }\end{array}$ & & $\begin{array}{c}590 \\
4-21-86\end{array}$ & $\begin{array}{c}593 \\
4-31-36\end{array}$ & $\begin{array}{c}594 \\
4-22-2 b\end{array}$ & $\begin{array}{c}596 \\
4-21-36\end{array}$ & $\begin{array}{c}.597 \\
4-? 2-36\end{array}$ & $\begin{array}{c}599 \\
4-22-96\end{array}$ \\
\hline PARAMETEẼ̂ & UNITS & & & & & & \\
\hline BICÄRBONATE & MGiL & 174 & 164 & 55 & 162 & 122 & 251 \\
\hline CHLCRIDE & U6/ML & $! 3$ & 11 & 13 & 17 & 10 & 45 \\
\hline FLUOR!aE & UE/ML & $<1$ & $\ll 1$ & $〈 1$ & $\langle!$ & 1 & $<1$ \\
\hline NITRATE & $U E / M L$ & $<5$ & $<5$ & $<5$ & $<5$ & $<5$ & $<5$ \\
\hline SULFATE & $U E / M L$ & 30 & 24 & 38 & 20 & 29 & 54 \\
\hline ÁLUAINUM & J6inL & 1 & 11 & 0 & 8 & 3 & 1 \\
\hline BARILH & UG/ML & 0 & 1 & ) & 0 & 1) & 0 \\
\hline CalciUn & UE/AL & 120 & 120 & 94 & 95 & 110 & 140 \\
\hline IRON & U6/ML & 1 & 11 & 0 & 4 & 3 & 1 \\
\hline MAGHESIUH & UG/AL & 10 & 6 & 6 & 10 & 8 & 24 \\
\hline MANGANESE & UE/AL & 0 & 9 & 3 & 1 & 1 & ) \\
\hline SCDIUH & U6iKL & 10 & 8 & 7 & $1 !$ & 10) & 11 \\
\hline SiL!CON & U6/ML & 4 & 10 & 4 & 12 & 5 & b \\
\hline STRONTIUA & U6/KL & 0 & 0 & 0 & 0 & 0 & 1 \\
\hline ZIHC & U6/ $/ \mathrm{LL}$ & 0 & 1) & $\ll 0$ & 0 & 0 & $<0$ \\
\hline EROSS ALPHA & $\mathrm{BO} / \mathrm{L}$ & 2.141 & 0.0631 & 0.0791 & 0.062 & 0.163 & 0.112 \\
\hline GROSS SETA & BQIL & 85.925 & 0.2004 & 0.6205 & 0.223 & 6.303 & 0.414 \\
\hline TRITIUH & $B Q / L$ & 3.56 & 0.148 & $2.121:$ & 0.156 & 0.214 & D. .444 \\
\hline TOC & UG/AL & 3 & 3 & 4 & 1 & 4 & 2 \\
\hline CARBON TETRACHLDRIDE & UG/L & $\langle 3$ & 3 & ij & 10 & $\langle 3$ & 13 \\
\hline CHLOROFORM & $46 / L$ & 8 & 4 & $<2$ & 44 & 50 & $<2$ \\
\hline DICHLOROBROMOMETHANE & U6 $\mathrm{h}$ & $\vdots 2$ & $<2$ & $<2$ & 12 & 5 & $<2$ \\
\hline 1.1-DICHLOROETHENE & UG/L & $<3$ & $\langle 3$ & $<3$ & $<3$ & $\langle j$ & $<3$ \\
\hline TETRACHLGROETHENE & UG/L & 108 & $<t$ & 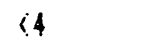 & $<4$ & $<4$ & $<4$ \\
\hline TOLLENE & U6/L & EST & EST<S & EST<S & ESTIS & EST $<5$ & EST $\angle S$ \\
\hline AETHYLENE CHLGRIDE & $46 / \mathrm{L}$ & 13 & $\langle 3$ & 5 & (j) & 17 & 3 \\
\hline IRAHS-1,2-DICHLOROETHENE & UGiL & 7 & 12 & $\langle 2$ & $\langle 2$ & $<2$ & 42 \\
\hline TRICHLOROETHEME & $U 6 / L$ & 71 & i2 & 62 & 7 & 42 & $<2$ \\
\hline ACETONE & IJGil & NT & NT & NT & AT & NT & NT \\
\hline GDISULFIÖE & US/L & NT & HT & NT & NT & NT & NT \\
\hline PH & 5To & 7.3 & 7.1 & 6.9 & 7.2 & 7.3 & 7.4 \\
\hline IEMFERATURE & c & 16.5 & 16.3 & $! 7.7$ & 22.9 & 15 & 14.1 \\
\hline CCNDUCTIYITY & UHHOS & 120 & 90 & 140 & 140 & 50 & 230 \\
\hline
\end{tabular}


'AEL! MO.

DATE JAMPLED

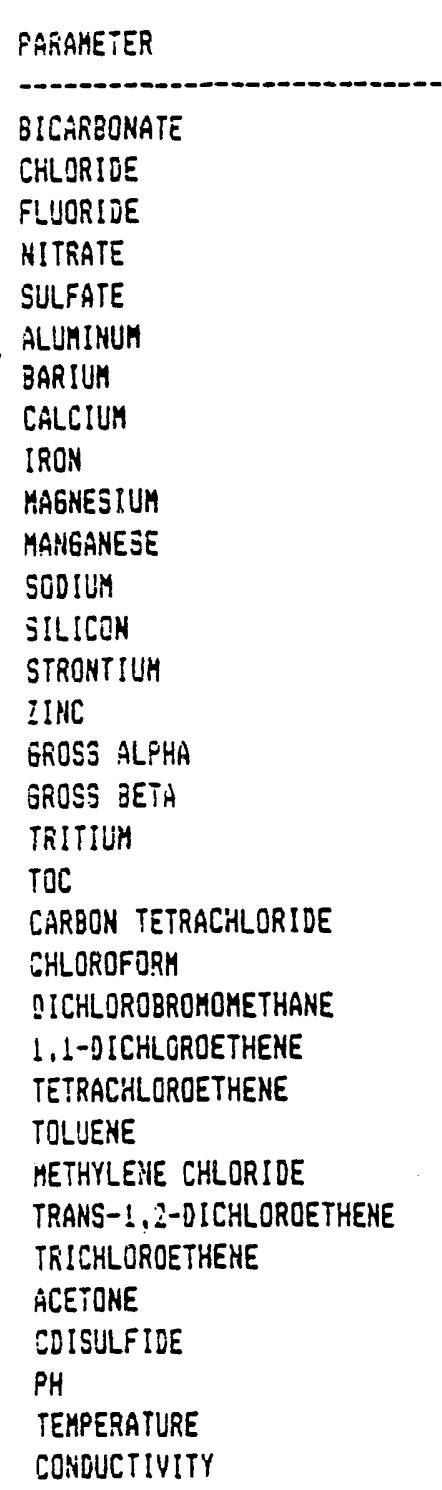

$$
\begin{array}{cccccc}
600 & 502 & 604 & 508 & 510 & 511 \\
10-15-36 & 4-50-36 & 4-29-86 & 5-59-36 & 5-11-86 & 4-30-96
\end{array}
$$

UNITS

ME/L

UG/AL

UGIAL

UG/HL

UG/ML

UG/ML

UG/ML

UG/XL

UG/ML

UESIAL

UG/AL

U6/ML

U6/ML

U6/AL

UE/ML

BQ/L

BQ/L

$30 / L$

U6/ML

UGiL

$U 6 / L$

UG/L

UG/L

UG/L

UG/L

U6/L

UG/L

UG/L

$U G / L$

U6/L

STD

c

UMHOS

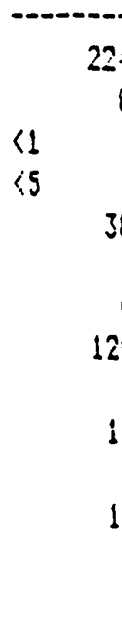

(0)

0.129

1.129

52.205

NT

$<5$$$
45
$$$$
<5
$$$$
\text { <5 }
$$$$
<5
$$

i5

$<4$

$$
5.4
$$

$\begin{array}{rr} & 172 \\ & 30 \\ 15 & \\ & \\ & 50 \\ & 1 \\ & 0 \\ & 79 \\ & 1 \\ & 14 \\ & 0 \\ & 25 \\ 9 & \\ & 1 \\ 0.119 & \\ 0.298 \\ 0.354\end{array}$

i1

22

4

$$
300
$$

1

10

4

16

8

$\begin{array}{rr}0 & 0 \\ 0.11 & 0.119 \\ 0.294 & 0.298 \\ 0.526 & 0.354 \\ 3 & 2\end{array}$

$\begin{array}{llll} & <3 & & 13 \\ & 3.4 & <2\end{array}$

12

3

$<3$

38
7.4

15.5

280

\section{ESTIS EST $\angle 5$}

$<3$

$<2$

12

NT

HT

8.2

20.2

360

$\begin{array}{rr} & 261 \\ 4 & 24\end{array}$

$<5$

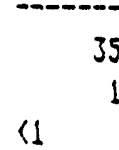

is

10

13

i1

339

11

87

1

170

2

19

2

25

7

0

$0.0694 \quad 0.126 \quad 0.193$

2.0817 .

1.701

1.775

$20.7836 \cdot 75.842$

0.485

$<3 \quad<3$

$<2 \quad<2$

$12 \quad \vdots 2$

29

$14<4$

EST $<5$

$<3$

$<2$

12

NT

8.1
25

130

NT

3.2
27.1
300

8.2

20.3

7.2

21.5

660 
Attachment B, Part A - RCRA Specific Comments Response 58

TITIE :

DOCUMENT NUMBER:

REVISION NUMBER:

DATE:

\section{SAMPIE ARCHIVING}

1605

A

$07 / 08 / 88$

PROJECT: ORNL RI/FS

JOB NO.: 19118

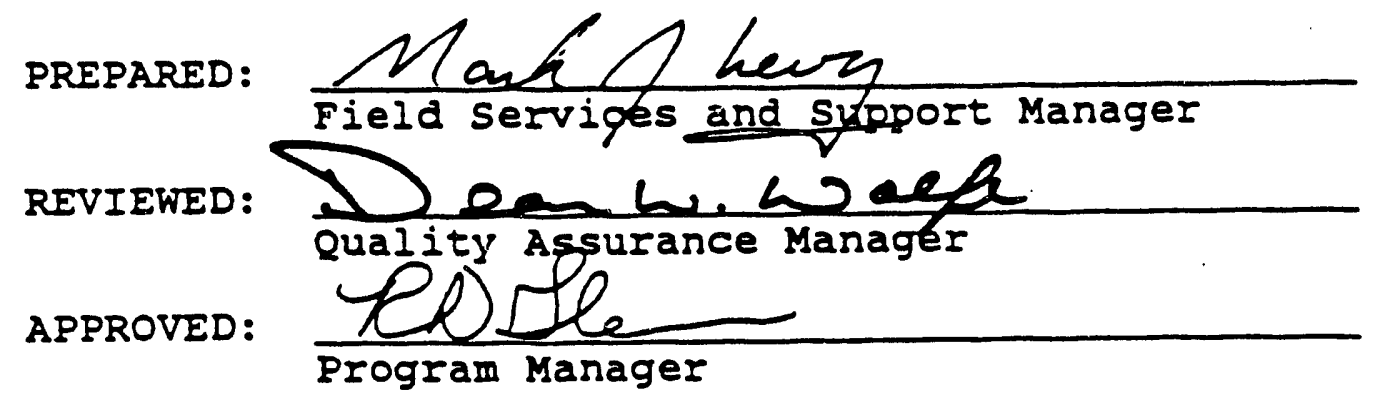

$7-7 \cdot 88$ Date $\frac{7-1 / 88}{\text { Date }}$ $\frac{7 / 11 / 88}{\text { Date }}$ 
Attachment B, Part A - RCRA Specific Comments Response 58

$\begin{aligned} 19118 \text { PP: } & 1605 \\ \text { Rev: } & \text { A } \\ \text { Date: } & 07 / 08 / 88 \\ \text { Page: } & 1 \text { of } 3\end{aligned}$

\section{SAMIPIE ARCHIVING}

\subsection{PURPOSE}

This procedure defines the protocols for executing sample storage at the designated $\mathrm{K}-25$ repository. The QA objective is to maintain sample integrity and chain of custody for 100 percent of the samples stored and to provide for efficient sample retrieval when necessary.

\subsection{SCOPE}

This procedure applies to all ORNI RI/FS project sample archiving.

\subsection{DEFINITIONS}

None.

\subsection{PROCEDURE}

4.1 GENERAL

The Sample and Analytical Laboratory (SAL) Coordinator will track the placement of all samples into either: 1) interim storage at the Field operations Facility (FOF), 2) storage after field screening at the FOF, or 3) storage after laboratory analysis at the remote analytical laboratory. on at least a quarterly basis, or more frequently if sample volume dictates, the SAI Coordinator will determine what samples have been in storage for a minimum of six months. The SAL Coordinator will then issue a sample Archiving Notification (SAN) form listing those samples six months or older to the FSS Manager, respective WAG managers, and to the Analytical Laboratory Subcontractor (AIS) to alert them of the upcoming archiving event. Within seven days, the WAG managers will return the SAN form to the SAL Coordinator, appropriately noting those samples listed on the SAN form which should not be archived.

In the event that the AIS's radioactive materials inventory approaches their regulatory limit, the AIS will notify the SAI coordinator of the need to initiate an archiving event or identify those samples less than 6 months old that would be potential candidates for the next archiving event. 
Attachment B, Part A - RCRA Specific Comments Response 58

$\begin{aligned} 19118 \text { PP: } & 1605 \\ \text { Rev: } & \text { A } \\ \text { Date: } & 07 / 08 / 88 \\ \text { Page: } & 2 \text { of } 3\end{aligned}$

\subsection{PACRAGING}

For those subject samples in which custody has not been transferred to the AIS, the SAL Coordinator will be responsible for packaging similar sample types from each WAG into appropriate storage containers. The SAL Coordinator, working in concert with the FHS Supervisor, will determine the appropriate container to be used. Each storage container will have a bar code label (ID number): and, using the Sample Information Management system (SIMS) sample archiving module (PP 1603), the ID for each storage container and the ID for each sample placed therein will be recorded. The container will be packed, placarded, and labeled for transportation in accordance with PP 1260, "Shipment of Hazardous Materials." The SAI Coordinator will direct the ALS to similarly package their subject samples for archiving. For those samples at the FOF, the FSS Manager will be responsible for the packaging of the samples, and the FHS supervisor will be responsible for the technical portion of the shipping consistent with PP 1260.

\subsection{SHIPPING}

After the SAL Coordinator has the sample shipment ready, including that portion from the analytical laboratory, the FSS Manager will be notified. The Energy systems counterpart will subseguently be notified, and a delivery time at the $k-25$ facility will be arranged. The samples will be shipped in accordance with PP 1260. Custody transfer will be documented as described by PP 1603, "Sample Information Management System." Upon arrival at the $\mathrm{K}-25$ facility, the vehicle and shipping containers will be surveyed and certified by the FHs supervisor. The shipment will then be escorted to the appropriate location in accordance with Energy Systems requirements.

\subsection{ARCHIVING}

Within the K-25 storage facility, each WAG will have a designated storage area. The FSS Manager, in concert with the SAI Coordinator, will develop the storage area plan. prior to shipment, after the type and number of the storage container has been determined, the FSS Manager will develop a detailed plan for the placement of the containers. Containers will be placed in accordance with that plan, and the actual location within the storage area will be recorded using the SIMS sample archiving module, noting such details as row and shelf designation. In addition, a logbook entry shall be made describing the placement

PP1605.WP 
Attachment B, Part A - RCRA Specific Comments Response 58

$$
\begin{aligned}
19118 \text { PP: } & 1605 \\
\text { Rev: } & \text { A } \\
\text { Date: } & 07 / 08 / 88 \\
\text { Page: } & 3 \text { of } 3
\end{aligned}
$$

of each container, using a sketch, if appropriate, made by the Archiving Team Leader.

\subsection{UPLOADING BAR CODE READERS TO COMIPUTERS}

The information collected by the hand-held bar code reader must be uploaded to RI/FS project PCs in accordance with PP 1603, "Sample Information Management System."

\subsection{RESPONSIBILITIES}

The Program Manager is responsible for ensuring overall compliance with this procedure.

The WAG manager is responsible for deciding what samples require archiving and when they should be archived.

The SAL Coordinator is responsible for identification of potential samples for archiving, issuing the SAN form to the WAG managers, assimilation and packaging of the appropriate samples, coordination with the ALS, shipment, and chain of custody requirements.

The FSS Manager is responsible for coordinating/managing packaging and shipment activities and access to the $\mathrm{K}-25$ facility.

The FHS supervisor is responsible for survey of shipments, completion of shipping papers, technical review of outgoing shipments, and review of ALs shipping procedures for compliance with project procedures.

\subsection{REFERENCES}

None.

7.0 ATTACRMENTS

None.

PP1605.WP 
TITIE:

DOCUMENT NUMBER: $\quad 1220$

REVISION NUMBER: A

DATE :
INITIAL SITE SURVEY

$06 / 30 / 88$

PROJECT: ORNI RI/FS

JOB NO.: 19118

PREPARED:

REVIEWED:

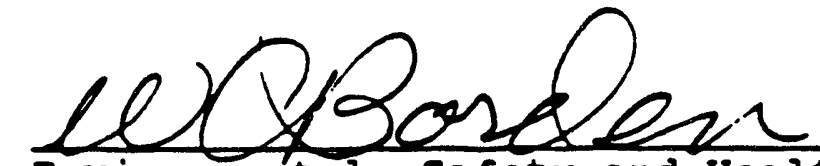
Environmental, Safety and Health Manager APPROVED: $7 / 1 / 80$ $7 / 1 / 82$ $\frac{7 / 1 / 88}{\text { Date }}$ 
Attachment B, Part B - RCRA Specific Comments Response 6

$$
\begin{aligned}
19118 \text { PP: } & 1220 \\
\text { Rev: } & \text { A } \\
\text { Date: } & 06 / 30 / 88 \\
\text { Page: } & 1 \text { of } 7
\end{aligned}
$$

\section{INITIAI SURVEY}

\subsection{ZURPOSE}

This procedure establishes the requirements for conducting initial surveys of potentially hazardous sites.

\subsection{SCOPE}

This procedure applies to 11 BNI and BNI subcontractor personnel who work on the ORNL reservation. This procedure applies only to those actions necessary to conduct initial surveys. Continuous, routine monitoring of work areas and the surrounding environment. is addressed by $P P$ 1255, "Work Area and Environmental Monitoring."

\subsection{DEFINITIONS}

Initial survey - Primary evaluation of a specified site, WAG, or other logical area for the detection of safety and health hazards. The initial survey consists of the preliminary evaluation and the entry survey.

\subsection{PROCEDURE}

Identification of chemical, biological, and physical hazards are conducted in accordance with 29 CFR 1910.120, "Hazardous Waste Operations and Emergency Response; Interim Final Rule" (Reference 1). Investigations of hazardous conditions are consistent with the type of operations planned. The ES\&H staff shall initially assess site hazards in three basic types of surveys:

- Initial survey - Prior to entry by RI/FS personnel other than those responsible for the entry survey

- Characterization - Remedial investigation characterization surveys

- Pre-work - Prior to RI/FS work in an area, especially activities that might change site conditions and/or present additional or difierent site hazards, such as drilling or digging

PP1220.WP 
Attachment B, Part B - RCRA Specific Comments Response 6

$\begin{aligned} 19118 \text { PP: } & 1220 \\ \text { Rev: } & \text { A } \\ \text { Date: } & 06 / 30 / 88 \\ \text { Page: } & 2 \text { of } 7\end{aligned}$

\subsection{PERSONAL PROTECTIVE EQUIPIENT AND ADMINISTRATIVE CONTROLS}

Personal protective equipment (PPE) requirements for initial surveys are determined based upon information obtained during data review and interviews as outlined in section 4.3. PPE levels as outlined in PP 1230, "Personal Protective Equipment," are used. In addition, escape devices (self-contained breathing apparatus of at least five-minute duration) shall be carried by entry survey personnel as required by 29 CFR 1910.120, "Hazardous Waste Operations and Emergency Response; Interin Final Rule" (Reference 1). Continuous monitoring for external radiological and other hazards is conducted in accordance with Paragraph 4.3 of this procedure. specialized hazardous chemical monitoring and use of specialized radiological dosimetry shall be used as conditions warrant or as determined by the FHS supervisor.

The minimum crew size for the conduct of entry survey activities shall consist of two individuals. Each pair of individuals shall be equipped with a reliable two-way radio or other communications device capable of contacting ORNL emergency personnel as outlined in PP 1240, "Emergency Response."

\subsection{INITIAL SURVEYS}

Initial surveys are conducted to examine existing records, inspect on-site conditions, investigate the presence of containers or obvious indicators of the presence of hazardous materials, measure ambient air or areas for the presence of toxic chemicals, or radiological hazards, and to allow safety and health personnel to become familiar with the site. Initial surveys must be conducted prior to the onset of any other field activities. Initial surveys must be conducted by qualified ES\&H personnel as determined by the ES\&H Manager in a minimum of three phases: interviews/record reviews, perimeter and on-site visual observations, and hazardous material surveys.

\subsubsection{Preliminary Evaluation}

The first phase of initial surveys is the review of existing records and interviews of appropriate ORNL health and safety personnel. ES\&H personnel review existing RI Plans which contain summary information concerning the hazardous materials expected to be present in the area and any other relevant information discovered since RI Plan development. ES\&H personnel also interview appropriate ORNL health and safety personnel to ask questions concerning unusual hazards that may be

RP1220.พT 
Attachment B, Part B - RCRA Specific Comments Response 6

$\begin{aligned} 19118 \text { PP: } & 1220 \\ \text { ReV: } & \text { A } \\ \text { Date: } & 06 / 30 / 88 \\ \text { Page: } & 3 \text { of } 7\end{aligned}$

present in the area to be surveyed. These interviews will be coordinated by the ES\&H Manager with the Energy Systems ES\&H Coordinator. Information gathered by record reviews and interviews will be discussed thoroughly by the Es\&H Manager for designee) or qualified ES\&H personnel prior to any physicil surveys that will take place. These pre-job briefings will be mandatory on all initial site surveys.

\subsubsection{Visual Observations}

The second phase of the initial survey consists of perimeter and on-site visual observations. Es\&H personnel must obtain a site map prior to conducting the visual observation phise of the initial survey. Visual observations or discrepancies between site conditions and existing maps should be noted in logbooks and on the drawing. ES\&H personnel must note the minimum following conditions as may be applicable:

- Containers and their condition (e.g.. tanks, pits, wells, drums, basins, ponds, piping, conduits); containers are not to be opened

- Abandoned equipment or structures

- Old markings, signs, labels, placards

- Biologic indicators (e.g., dead animals, plants)

- Unusual conditions (e.g., clouds, discolored liquids, oil slicks, vapors, stained soils, debris, disturbed soil)

- Odors (usually reason to leave the site)

- other unusual conditions

\subsubsection{Hazardous Chemical Surveys}

Qualified ES\&H personnel bill monitor suspected areas, at a minimum, for the following hazardous conditions/substances:

- Total organics with a portable organic vapor analyzer

- Oxygen levels

- Combustible gas

PP1220,WP 
Attachment B, Part B - RCRA Specific Comments Response 6

$\begin{aligned} 19118 \text { PP: } & 1220 \\ \text { Rev: } & \text { A } \\ \text { Date: } & 06 / 30 / 88 \\ \text { Page: } & 4 \text { of } 7\end{aligned}$

- Suspected volatile or gaseous inorganics with direct reading instruments

- Windspeed, temperature, humidity, and wind direction (as appropriate)

The hazardous chemical survey shall be undertaken as required by 29 CFR 1910.120 to identify conditions that may cause death or serious harm and to identify and quantify airborne levels of hazardous materials. The procedures are similar to those of a radiological walkover survey discussed below, with the addition of the instruments listed above.

Results of hazardous chenical surveys will be documented using forms provided in PP 1270.1, "Air surveillance of Radiological and Chemical Contaminants." As conditions warrant, samples of soil, water, or other environmental media may be collected on a limited basis to further define site hazards.

\subsubsection{Radiological Surveys}

ES\&H personnel should scan accessible areas for exposure rates and make contamination measurements (fixed and removable) of any suspicious areas. The radiological surveys will be performed in accordance with PP 1280.5, "Dose Rate Measurements," and PP 1280.1, "Methods for Performing Radiological Contamination surveys." As conditions warrant, samples of soil, water, or other environmental media may be collected on a limited basis to further define site hazards.

\subsection{CHARACTERIZATION SURVEYS}

The characterization surveys referred to herein are those area surveys specified by the RI Plan and performed primarily to gather data for the RI. The information collected will be used to prioritize sampling and as an indication of cortaminant migration.

\subsubsection{Radiation Walkover Survey}

A radiation walkover survey is the measurement of the radiation levels throughout an area for the purpose of estimating the areal extent of surficial soil contamination and locating radiation anomalies which may indicate the presence of surficial or subsurface contamination. The following are measured during the walkover survey:

PP1220.WP 
Attachment B, Part B - RCRA Specific Comments Response 6

$\begin{aligned} 19118 \text { PP: } & 1220 \\ \text { Rev: } & \text { A } \\ \text { Date: } & 06 / 30 / 88 \\ \text { Page: } & 5 \text { of } 7\end{aligned}$

- Gamma radiation from the soil

- Beta radiation at the soil surface

- Exposure rate at 1 meter above the ground

The instrument/probe combinations which may be used for a walkover survey are listed below categorized by use. Preferred combinations are marked with an asterisk (*). The other combinations would be substituted whenever the chosen combination becomes ineffective due to saturation or high background.

- Gamma radiation from soil, listed in order of decreasing sensitivity:

- Count rate meter with shielded gamma scintillation probe

- Count rate meter with gamma scintillation probe

- Count rate meter with low-range compensated GM probe

- Count rate meter with high-range compensated GM probe

- Ionization chamber meter

NOTE: A variation of the count rate meter with gamma scintiliation probe which may be used is the Ultrasonic Ranging and Detection System (USRADS).

- Exposure rate, listed in order of decreasing sensitivity:

- *Pressurized ion chamber

- Count rate meter with gamma scintillation probe

- Count rate meter with low-range compensated GM probe

- Count rate meter with high-range compensated GM probe

- Ionization chamber survey meter

- Surface beta levels, listed in order of decreasing sensitivity:

- Count rate meter with shielded pancake GM probe.

- Count rate meter with unshielded pancake GM probe.

- Count rate meter with end window GM probe.

Prior to the radiation walkover survey but following the preentry survey, a civil survey team will establish a grid covering the entire area, typically a square grid with 100-ft spacing. Each grid intersect point will be marked with a stake and

PP1220.WP 
Attachment B, Part B - RCRA Specific Comments Response 6

$$
\begin{aligned}
19118 \text { IP: } & 1220 \\
\text { ReV: } & \text { A } \\
\text { Date: } & 06 / 30 / 88 \\
\text { Page: } & 6 \text { of } 7
\end{aligned}
$$

coordinates. Where the grid intersect points are obstructed (e.g., by a building), additional stakes will be placed in convenient locations; i.e., at building corners.

The walkover survey will be performed under the following conditions:

- The survey team shall consist of at least two people.

- The area to be surveyed should be subdivided into sections which can easily be surveyed in a day. These sections should be groups of grid squares (i.e., $100 \times 100-f t$ areas with grid stakes at each corner for a 100-ft grid).

- The pre-entry survey conducted by ES\&H personnel must be reviewed to determine if a Hazardous work Permit (HWP) (PP 1235) is required, the PPE required, the general conditions of the site, and aid in selection of the survey instruments.

- Site maps, with the survey grid, should be prepared to aid in data collection.

- Before each day's use and periodically during the day, survey instruments must be source checked for proper operation and current calibration.

- Instruments to be used for measuring the gamma radiation levels from soil will be configured to allow the probe (detector) to be positioned at a uniform distance from the surface (as close as practical, usually three to four inches). This is accomplished for gamma scintillation probes by attaching a cord to the probe so that the operator can suspend the probe over the surface to be surveyed.

- When scanning, the probe should not be moved across the area at speeds greater than two feet per second. Faster speeds may cause small spots of elevated radiation levels to be missed.

- If the USRADS is used, it shall set up according to the instructions provided with the system and its use directly supervised by a trained individual.

- The measurements at fixed points of the exposure rate and surface beta should be made at the grid points associated with the areas scanned, and at locations within that area

PP1220.WP 
Attachment B, Part B - RCRA Specific Comments Response 6

$$
\begin{aligned}
19118 \text { PP: } & 1220 \\
\text { Rev: } & \text { A } \\
\text { Date: } & 06 / 30 / 88 \\
\text { Page: } & 7 \text { of } 7
\end{aligned}
$$

which demonstrated elevated radiation levels. Measurements of the radiation levels from soil should be made at the same locations.

\subsection{PRE-WORR SURVEY}

The pre-work survey is essentially the same as the pre-entry survey, except for the following:

- It occurs after the pre-entry survey

- It generally covers a smaller area

- The protective equipment requirements are better defined

- It collects data that will be used in controlling a specific type of work through issuance of an HWP, if required

- It identifies data that may be of concern to ES\&H personnel doing pre-work briefings, if required

\subsection{RESPONSIBILITIES}

The Program is responsible for ensuring overall compliance with this procedure.

The ES\&H Manager is responsible for ensuring compliance with this procedure and for assigning personnel to perform dosimetry program support functions.

The FHS Supervisor is responsible for field implementation of this procedure.

\subsection{REFERENCES}

(1) U.S. Code of Federal Requlations, 29 CFR 1910.120, "Hazardous Waste Operations and Emergency Response; Interim Final Rule," December 19, 1986.

\subsection{ATTACTREENTS}

None. 
FINAL REPORT Response 7

BECHTEL ID NUMBER:

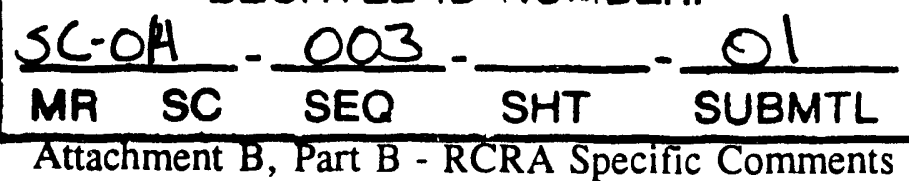

USRADS SURVEY OF WASTE AREA GROUPING 1

WORK RELEASE $X-17$

\subsection{INTRODUCTION}

This report documents the ultrasonic Ranging and Data system (USRADS) walkover survey of the Oak Ridge National Laboratory (ORNL), Waste Area Grouping 1 (WAG-1). The survey was initiated under subcontract \#19118-SC-014, work release X-17. The walkover survey began on March 26, 1990 añd was terminated on July 10, 1990. During this time frame, 177 surveys were completed. The actual time spent in the field surveying was 69 days.

Three distinct field survey measurements were collected and documented. One hundred and sixty-seven ground surface contamination and area dose rate measurements were collected simultaneously during a one time walkover survey. Ten electromagnetic ground conductivity measurements were taken separately at selected locations within the WAG-1 boundary. summary statistical data for the survey are listed in Table 1 along with the production rates for the project.

Table 1. Survey summary statistics.

\begin{tabular}{|c|c|c|c|c|}
\hline MONTH & SURVEYS DAYS & NUMBER OF SURVEYS & \multicolumn{2}{|c|}{ GRIDS } \\
\hline March & 5 & \multicolumn{2}{|l|}{11} & 11 \\
\hline April & $\begin{array}{l}16 \text { (Rad) } \\
4.5(E M-31)\end{array}$ & \multicolumn{2}{|c|}{$\begin{array}{l}46(\operatorname{Rad}) \\
8(E M-31)\end{array}$} & ${ }^{34} 5.5$ \\
\hline May & $19(\operatorname{Rad}))$ & \multicolumn{2}{|c|}{ 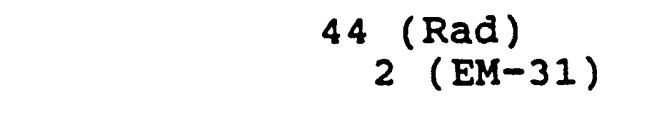 } & 44 \\
\hline June & 17.5 & \multicolumn{2}{|l|}{51} & 51.5 \\
\hline July & 6 & \multicolumn{2}{|l|}{15} & 12 \\
\hline TOTAL & $\begin{array}{l}63.5(\operatorname{Rad}) \\
5.5(\mathrm{EM}-31)\end{array}$ & \multicolumn{2}{|c|}{$\begin{array}{l}167(\operatorname{Rad}) \\
10(\mathrm{EM}-31)\end{array}$} & $\begin{array}{r}156 \\
5.5\end{array}$ \\
\hline PRODUC & $\begin{array}{r}N \operatorname{RATES}(\operatorname{Rad}): \\
(\operatorname{EM}-31):\end{array}$ & $\begin{array}{c}2.6 \mathrm{SRV} / \mathrm{DAY} \\
1.8 \mathrm{SRV} / \mathrm{DAY}\end{array}$ & 2.5 & $\begin{array}{l}\text { GRIDS/DAY } \\
1 \text { GRIJ/DAY }\end{array}$ \\
\hline
\end{tabular}


Due to the large quantity of data, representing several computer printouts per survey, three volumes were required to contain all the data. All the computer data plots can be found in Appendix $C$, Summary Grids. The survey data is divided into 23 summary Grids. The Summary Grids can be located by the tabs on the side of each volume. A site map showing all the summary Gridis can be found at the front of Appendix $C$.

Each Summary Grids contains a composite track map with the appropriate coordinates and grid identification numbers. A list of findings relative to each survey can be found in the front of each Summary Grid along with a listing of the survey, coordinates of each survey and the dates the surveys were performed. The electromagnetic ground conductivity survey data is compiled separately in Appendix D.

\subsection{DESCRIPTION OF THE SURVEY SITE}

The WAG-1 site includes the major portion of the ORNL Main Plant area. The area identified for the USRADS surveys encompassed approximately 170 acres inside and outside of the main plant area. The general boundaries of the walkover survey are: Bethel valley Road on the north: White Oak Creek on the south; First Creek on the west; and the limits of the main security fence on the erist.

A 200 by 200 foot survey grid was established within the boundaries of the ORNL site prior to initiation of the USRADS surveys. The 170 acre site is approximately equivalent to 187 of the 200 by 200 foot grid blocks.

\subsection{USRADS AND SURVEY INSTRUMENT CONFIGURATION}

\subsection{DESCRIPTION OF THE USRADS SYSTEM}

The USRADS is specifically designed to automatically correlate signal input data with the geographical location of that data during walkover type surveys. The USRADS incorporates three technologies: radio frequency (RF) communications, ultrasonics and microcomputers. $R F$ is utilized for system timing and data transfer. Propagation time of an ultrasonic signal is used to measure distance. Microcomputers are used to collect data, calculate distances, data display, data storage, data reduction, report preparation, and data transfer into existing electronic databases. See Figure 1 for basic system intercommunications.

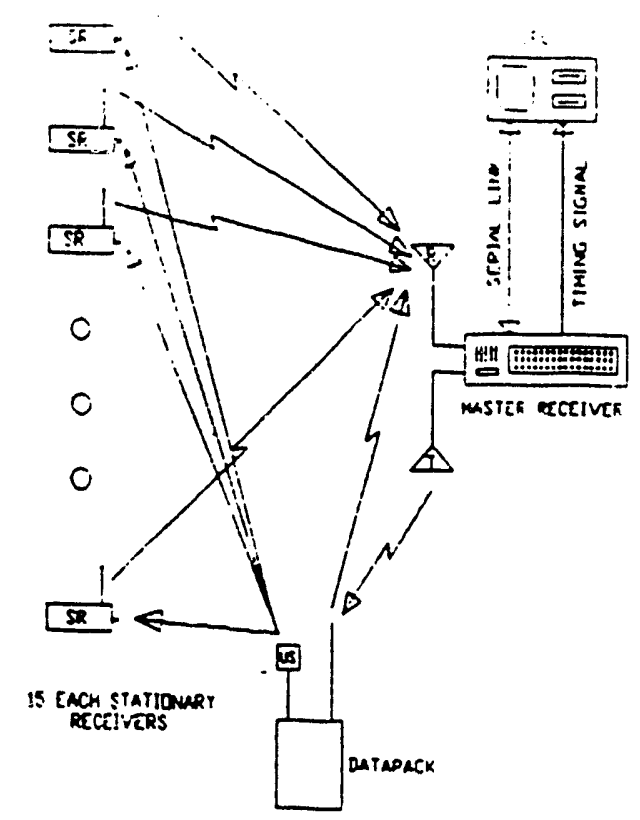

Figure 1 SYSTEM INTERCONNECTIONS 
Attachment B, Part B - RCRA Specific Comments Response 7

\subsubsection{Basic Methodology}

In USRADS, a unique ultrasonic signal is emitted from the surveyor's location each second. At precisely the same time, an RF transmission is sent to the microcomputer location. since the RF transmission is essentially instantaneous in its speed of travel when compared to the speed of sound, the RF transmission is used as the start time for the ultrasonic signal. Each stationary Receiver (SR) has a RF transmitter, as well as, an ultrasonic receiver.

When the SR identifies the unique ultrasonic signal, the SR transmits a RF signal to the microcomputer which is used as the stop signal of the ultrasonic transmission and, therefore, establishes the length of time it took for the sound to that SR. The microcomputer then computes the distance between the surveyor and that SR. As each SR responds to the ultrasonic signal, corresponding stop signals are sent and distances calculated. From this information, the location of the surveyor is established each second.

To accomplish the necessary correlation between the surveyor's location and the data signal input (radiation instrument signal or EM- 31 signal), the RF start signal is coded with the data collected from the last second. This automatically correlates the measurement with the proper location. As each one-second position is determined in the microcomputer, a dot is plotted on the microcomputer screen in correct relationship to the SRs that have been placed within the survey area. The data for that one-second time period is also posted at the bottom of the computer screen. The plotted position remains on the computer screen while the data collected is replaced each second to conserve screen space for the plotting of the track of the surveyor. At any time during the survey, the surveyor may look at the track line to determine if any areas have been missed, and, if they have, the surveyor may return to those areas and obtain the necessary coverage of the areas. In this manner, the surveyor can concentrate on obtaining full coverage of the survey area in a minimum amount of time.

When proper survey coverage has been accomplished, the surveyor may then run the data reduction routines on the microcomputer. several different software packages are resident so that the surveyor may review survey coverage and identify anomalies or other points of interest.

\subsection{SURVEY INSTRUMENTATION}

\subsubsection{Near Surface Gamma Detection Survey Instrument}

Near surface gamma detection surveys were conducted using a Victoreen Thyac model III, serial number 055088. The detector for this instrument is a 1 1/2-inch sodium iodide (NaI) scintillation crystal. 
The Thyac 3 is attached to the surveyor's backpack for ease of handling. The output signal of the Thyac 3 is directly interfaced to the USRADS e $q u$ i $p$ e $n t$. Therefore, the input signal to the USRADS is the same signal as that generated by the instrument detector. The sodium iodide de $t$ e c tor is electronically coupled to victoreen Thyac 3 count rate meter by coaxial cable. The detector is suspended from the surveyor's backpack by an attached boom. (See Figure 2.).

This arrangement

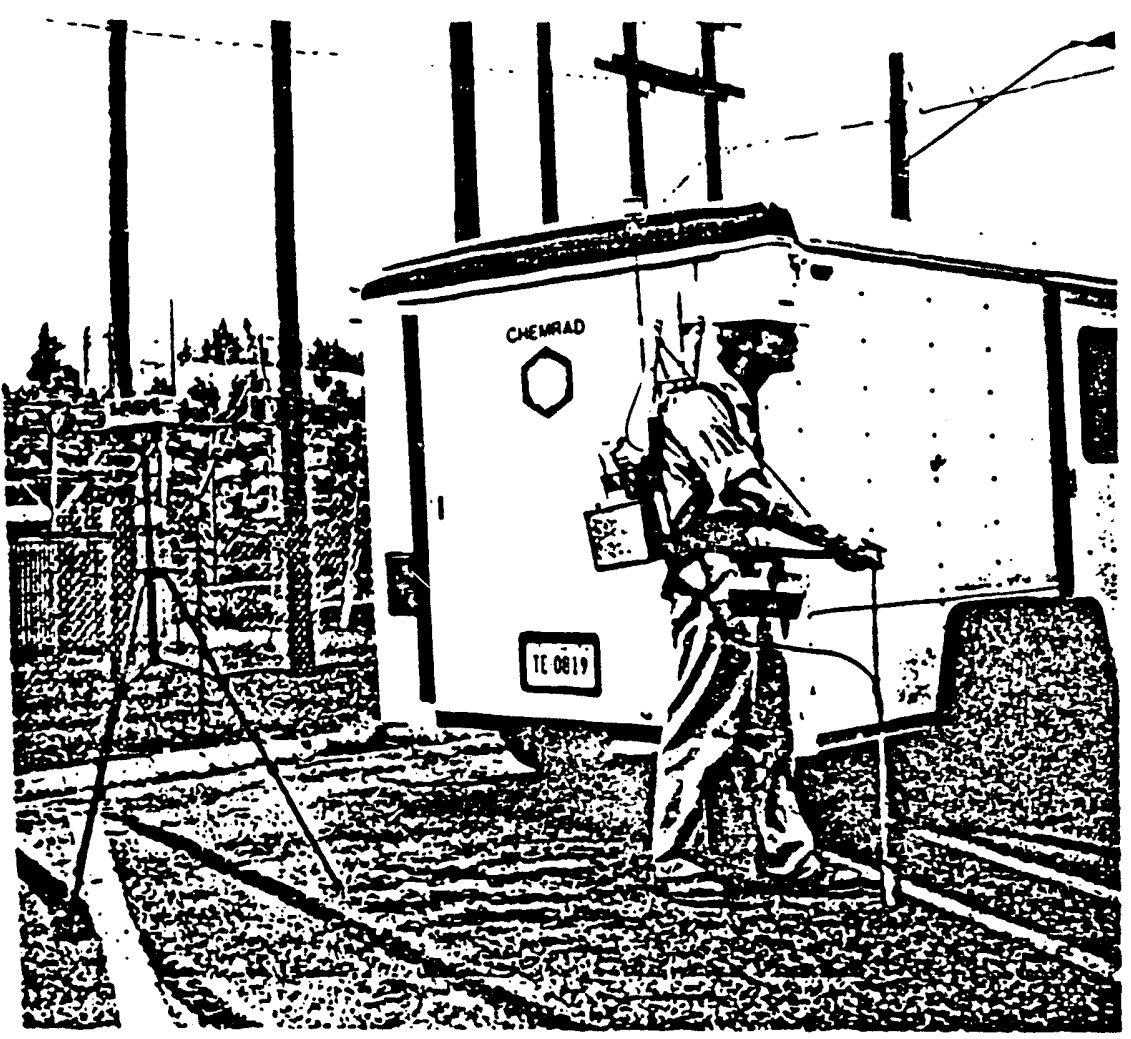

FIgure 2 SURVEY INSTRUMENTATION ARRANGEMENT.

enables the surveyor

to walk without holding onto the survey meter, swing the detector across the parallel intersects, and maintain approximately 15 centimeters between the detector and the ground. The output signal of the Thyac 3 is directly connected the USRADS electronics through a connector port on the Datapack face plate. Therefore, the counts per minute data recorded by the USRADS is the same signal generated by the detector.

\subsubsection{Gamma Exposure Rate Survey Instrument}

Gamma exposure rate surveys were conducted using a victoreen model 450-P pressurized ionization chamber, serial number 791 , with an optical interface. The Model 450-P was connected to the USRADS equipment through the optical interface. The optical interface reads the output signal of the ionization chamber and sends the signal to the USRADS via a RS-232 connection on the USRADS Datapack face plate. In this manner, the signal input to the USRADS is the same as that generated by the Model 450-P. The pressurized ion chamber was attached to the boom that suspended the NaI detector from the surveyors backpack (about one meter from ground surface). This configuration provided consistent geometry throughout the entire survey. 


\subsubsection{Electromagnetic Ground Conductivity Survey Instrument.}

The electromagnetic ground conductivity surveys were conducted using a Geonics Model EM-31-DL ground conductivity meter, serial number 07004 . The EM-31 was interfaced to the USRADS equipment through a cable connected to the RS-232 port on the Datapack front face plate. This configuration enabled the USRADS equipment to record both the quadrature phase and in-phase components simultaneously. The quadrature phase component gives the ground conductivity measurements in millimhos per meter. The in-phase component is used primarily in the EM-31 for calibration purposes. However, this component is significantly more sensitive to large metal objects and, therefore, very useful when looking for underground metal objects. It is important to note that the graphic representations of the in-phase survey data are unitless and the scaling factors on the graphics are an indication of the intensity of the measurement.

\subsection{SURVEY RESULTS}

\subsection{GROUND SURFACE CONTAMINATION AND DCSE RATE SURVEYS}

\subsubsection{Survey Methodology}

The ground surface contamination survey was performed by swinging the NaI detector across the surveyors path of travel in a slow motion, at a distance of approximately 15 centimeters from the ground surface. The width of the detector swing was approximately one meter. The surveyor walked transects back and forth across the survey area approximately 1-1/2 meters center-to-center. The rate of travel by the surveyor was approximately one meter per second.

\subsubsection{Explanation of Data}

The radiation surveys are divided into summary Grids (SG). Each SG is composed of a 600 by 600 foot segment site grid map, corresponding to nine 200 by 200 foot grid blocks. The SGs are arranged in 5 rows and are numbered sequentially, starting with SG 1 at the northwest corner of the site and ending with SG 23 at the south center of the map (See Figure 3 and front of Appendix C.)

Each SG contains a composite track map, survey location data, survey findings and a key to the obstacles encountered during each survey. The surveys are arranged according to the grid blocks in which they were performed. The computer plots of the survey data include track maps frr both CPM and $u R / h r$ survey; two dimensional contour and three dimensional plots when instrument data is above the threshold cutoff. In cases where the survey data is below the threshold cutoff, or when just a few data points are highlighted, statistical summary data is included.

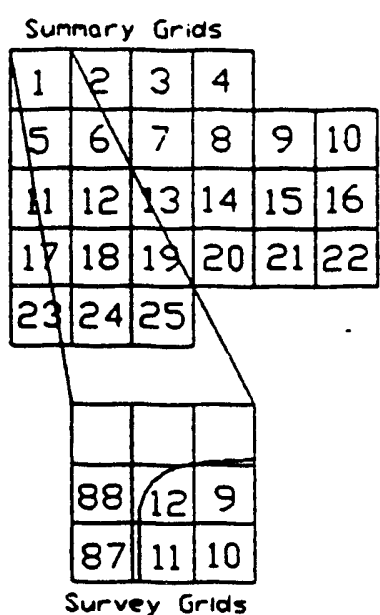

Figure 3 GRID NUMBERING SYSTEM 


\subsubsection{Problems Encountered During the Survey}

The problems encountered during surveying included environmental, site and equipment problems.

Environmental problems include rain, high wind and extreme temperatures. Rain and wind delayed surveys and affected equipment operation. Extreme temperatures created temperature differentials in the equipment which affected performance. Two days worked in July during extreme heat affected surveyors by forcing work times to be limited to 20 minutes with 20 minutes of rest. Total lost work time to environmental problems was 3.5 days.

Site problems encountered included steam leaks, air leaks and construction that created high frequency noise that affected equipment operation. A total of 8.5 grids were unable to be surveyed due to steam leaks. Another 3 grids were delayed in being surveyed due to noise sources in those areas. Actions taken to lessen the effects of high noise included repositioning stationary receivers or the boundaries of the survey area.

The steam plant supervisor was contacted in regards to the steam leaks to determine a schedule for the repair of the leaks. During the time surveys were being performed, approximately 3 leaks were repaired with the other leaks scheduled to be repaired in the future. Another problem encountered was the proximity of buildings and other obstructions in the survey area making setups and surveys difficult. Actions taken to solve this problem included overlapping survey grids or doing multiple setups to cover an area.

Equipment problems encountered included broken or loose wiring, weak or dead batteries and computer problems. Most equipment problems were corrected in the field to minimize time lost for surveying. The embedded computer in the datapack failing was the only equipment problem causing a full day to be lost. Total time lost due to equipment problems was approximately 2 days.

\subsection{PROBLEM AREAS}

Several problem areas were encountered on site that prevented surveys in those areas. These areas included areas between buildings and between buildings and fences. The following areas were not surveyed:

a. The area between building 2500 (Firehouse) and the outside fence:

b. The area between buildings 2525 and 2547;

c. The area between building 2506 and the contaminated pit to the south of the building;

d. The area between buildings 2525 and 2506;

e. The area surrounded by buildings $3550,3546,3500$ and 3606 ; 
Attachment B, Part B - RCRA Specific Comments Response 20

Method No.: ESP-900

Revision No.: 0

Date: August 27, 1988

Page 1 of 20

MARTIN MARIETTA ENERGY SYSTEMS, INC.

ENVIRONMENTAL SURVEILLANCE PROCEDURES

SUBJECT: CLEANING AND DECONTAMINATING SAMPLE CONTAINERS AND SAMPLING DEVICES

I. Scope and Application:

This procedure establishes methodologies for cleaning and decontaminating sample containers and sampling devices.

II. References:

A. Engineering Support Branch Standard Operating Procedures and Quality Assurance Manual, U.S. Environmental Protection Agency, Athens, GA, 1986.

B. Federal Regisier, Volume 44, 40 CFR Part 136. "Guidelines Establishing Test Procedures for the Analysis of Pollutants Under the Clean Water Act."

C. Test Methods for Evaluating Solid Waste, SW-846, 2nd Edition, U.S. Environmental Protection Agency, Washington, D.C., 1982.

III. Summary of Method:

Sampling containers used by field sampling teams may be obtained precleaned from commercial supplier, supplied by the supporting analytical laboratory, or prepared by the field team. Sampling devices must be cleaned prior to being used in the field to prevent potential contamination of a sample. Sampling devices must be cleaned and decontaminated between samples to prevent cross-contamination and must be decontaminated at the close of the sampling event prior to being taken off-site.

An acceptable aiternative to cleaning and decontaminating sampling devices is the use of items cleaned or sterilized by the manufacturer that are discarded after use. Care must be exercised to ensure such previously cleaned or sterilized items do not retain residues of chemical or radioactive sterilizing agents that might interfere with analytical techniques.

IV. Comments:

The cleaning materials referred to in this procedure are defined in the following paragraphs. 
Attachment B, Part B - RCRA Specific Comments Response 20

Method No.: ESP-900

Revision No.: 0

Date: August 27, 1988

Page: 2 of 20

MARTIN MARIETTA ENERGY SYSTEMS, INC.

ENVIRONMENTAL SURVEILLANCE PROCEDURES

\section{A. Definitions}

1. Sample containers include, but are not limited to, the following:

$\begin{array}{ll}0 & \text { Jars } \\ 0 & \text { Vials } \\ 0 & \text { Jugs } \\ 0 & \text { Air sampling tubes } \\ 0 & \text { Air sampling bags } \\ 0 & \text { Soil gas tubes } \\ 0 & \text { Soil sampling tubes } \\ 0 & \text { Air sampling filters. }\end{array}$

2. The laboratory detergent must be a standard brand of phosphatefree laboratory detergent such as Alquinox, Liquinox, or the equivalent.

3. The nitric acid solution (10 percent) is made from reagentgrade nitric acid and deionized or organic-free water.

4. The standard cleaning solvent will be pesticide-grade isopropanol. The use of any solvent other than pesticide-grade isopropanol for equipment cleaning purposes must be justified and approved by the responsible project personnel and will be documented in logbooks. The laboratory must be informed as well.

5. Tap water may be used from an approved municipal water treatment system. The use of an untreated potable water supply is not an accept ble substitute for tap water.

6. Deionized water is defined as tap water that has been treated by passing through a standard deionizing resin column. The deionized water should contain no heavy metals or other inorganic compounds (i.e., at or above analytical detection limits) as defined by a standard Inductively Coupled Argon Plasma Spectrophotometer (ICP) scan.

7. Organic-free water is defined as tap water that has been treated with activated carbon and deionizing units or water from a Milli-Q system (or equivalent). 
Attachment B, Part B - RCRA Specific Comments

Response 20

Method No.: ESP-900

Revision No.: 0

Date: August 27, 1988

Page: 3 of 20

MARTIN MARIETTA ENERGY SYSTEMS, INC.

ENVIRONMENTAL SURVEILLANCE PROCEDURES

B. General

During cleaning operations, the substitution of a higher grade water (i.e., deionized or organic-free water for tap water) is permitten and need not be noted as a variation.

The brushes used to clean equipment as outlined in the various sections of this procedure must not be of the wire-wrapped type.

The solvents, nitric acid solution, laboratory detergent, and rinse waters used to clean equipment must not be reused, except as specifically permitted.

V. Required Equipment and Apparatus:

Cleaning materials and equipment are outlined in Attachments 1 and 2.

VI. Safety

Safety glasses or goggles, gloves, and laboratory coat or apron will be worn during cleaning operations. Solvent rinsing will be conducted under a fume hood or in the open (never in a closed room). No eating, smoking, drinking, chewing, or hand to mouth contact will be permitted during cleaning operations.

VII. Procedure:

A. Select appropriate cleaning procedure from Attachments.

B. Segregation of Used Field Equipment:

Field equipment or reusable sample containers needing cleaning must not be stored with clean equipment, sample tubing, or sample containers. Field equipment, reusable sample containers, disposable sample containers, and sample tubing that are not used may not be replaced in storage without being recleaned if these materials are transported to a facility or study site where contamination or suspected contamination was present.

C. Storage of Cleaned Field Equipment and Sample Coniainers:

Previously cleaned sample containers and field equipment that are cleaned using the procedures outlined in the attachments are stored 
Attachment B, Part B - RCRA Specific Comments Response 20

Method No.: ESP-900

Revision No.: 0

Date: August 27, 1988

Page: 4 of 20

MARTIN MARIETTA ENERGY SYSTEMS, INC.

ENVIRONMENTAL SURVEILLANCE PROCEDURES

in a contaminant-free environment. Sample containers and field equipment are stored separately from all other equipment and supplies and from each other.

D. Transporting Used Sample Containers off-Site:

Sample containers that contain a sample, regardless of the assumed or known level of hazard associated with that sample, must have all exterior surfaces decontaminated. For sample containers used in areas other than a controlled access area, a wipedown with disposable rags or toweling, or rinse with deionized water followed by drying with disposable rags or toweling, will suffice. Any visible dirt, water droplets, stains, or other extraneous materials must be removed. For containers used in controlled access areas, a more rigorous cleaning and/or radiation monitoring may be required. Refer to the project specific work plan for details.

\section{Contamination Control:}

The solvent used to implement the cleaning procedures outlined in this method will be collected and disposed of by allowing to evaporate under a fume hood or be containerized and disposed per ESP-1000. Similarly, spent acids will be collected and disposed of by ESP-1000. These procedures apply whether cleaning procedures take place in the washroom or in the field.

IX. QA/OC:

A. General

This section outlines guidelines for spicific quality control procedures to monitor the effectiveness of cieaning procedures given in the attachments. Refer to the appropriate project work plans for more detail as necessary.

B. Rinse Water

The quality of the deionized and organic-free water used may be monitored by collecting samples in standard precleaned, sample containers and submitting them to the laboratory for a standard ICP scan. Organic-free water should be submitted for low level pesticide, herbicide, extractable, or purgeable compounds analyses as appropriate. 
Attachment B, Part B - RCRA Specific Comments Response 20

Method No.: ESP-900

Revision No.: 0

Date: August 27, 1988

Page: 5 of 20

MARTIN MARIETTA EÑERGY SYSTEMS, INC.

ENVIRONMENTAL SURVEILLANCE PROCEDURES

\section{Equipment Cleaning}

The effectiveness of the equipment cleaning procedures is monitored by submitting to the laboratory rinse water for low level analys is of the parameters of interest. An attempt should be made to select different pieces of equipment for this procedure, each time equipment is washed, so that a representative sampling (approximately 10 percent) of all equipment is obtained over the length of the project.
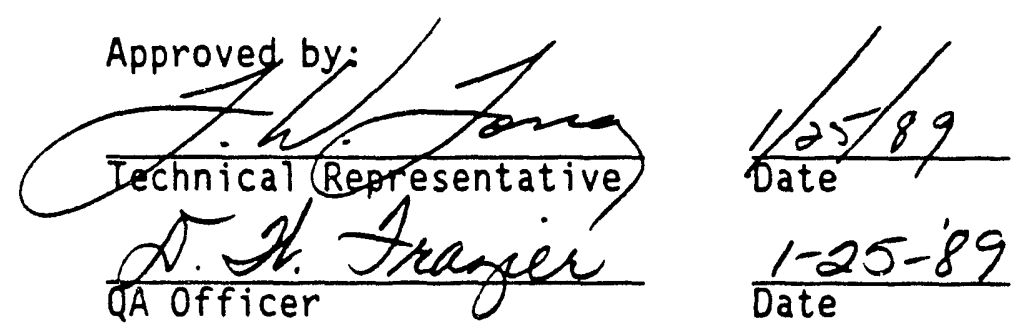
Method No.: ESP-900

Revision No.: 0

Date: August 27, 1988

Page: 6 of 20

MARTIN MARIETTA ENERGY SYSTEMS, INC.

ENVIRONMENTAL SURVEILLANCE PROCEDURES

\section{ATTACHMENT 1}

\section{Preparation of Disposable Sample Containers}

\section{A. General}

Disposable sample containers will be stored in their original packing containers. When packages of uncapped sample containers are opened, they will be stored to prevent contamination. Specific precleaning instructions for disposable sample containers are given in the following sections. These instructions apply to disposable sample containers whether they are purchased from an outside source or are precleaned by laboratory personnel.

\section{B. Cleaning of Sample Containers That Are Recommended For Use in the Analysis of:}

Acidity/Alkalinity

BOD (Biological oxygen Demand)

Chloride

COD (Chemical Oxygen Demand)

Color

Conductivity

Cyanide

Extractable Organics
Hardness

Mercury

Metals

$0 i l$ \& Grease Sulfide

pH

Phenols

Phosphate

Silica

Sulfate

Sulfite
Settleable Residue/Suspended Solids

TOC (Total Organic Carbon)

Turbidity

1. Wash bottles and jars, Tefion liners, and caps in hot tap water and laboratory detergent.

2. Rinse three times with tap water. 
Method No.: ESP-900

Revision No.: 0

Date: August 27, 1988

Page: 7 of 20

MARTIN MARIETTA ENERGY SYSTEMS, INC.

ENVIRONMENTAL SURVEILLANCE PROCEDURES

3. Rinse with nitric acid solution. ${ }^{1}$

4. Rinse three times with deionized water.

5. Rinse bottles, jars, and liners (not caps) with solvent. 1

6. Oven iry bottles, jars, and liners $\left(125^{\circ} \mathrm{C}\right.$. recommended) Allow to cool.

7. Place liners in caps and cap containers.

8. Store containers in contaminant-free area.

C. Cleaning of sample containers that are recommended for use in the analyses of:

COD

TOC

Nitrate-Nitrite

Trihalomethanes

Purgeable (Volatile) Organics

1. Wash containers, septa or liners, and closures in hot tap water with laboratory grade non-phosphate detergent.

2. Rinse three times with tap water.

3. Rinse three times with deionized water.

4. Oven dry containers, septa or liners, and closures.

5. Remove containers, septa, and closures from oven.

6. Place liners in closures, Teflon side down, and place on containers. - Attendant to wear gloves and containers cannot be removed from preparation room until sealed.

ISome vendors of precleaned, disposable sample bottles use pesticide grade methylene chloride to solvent rinse sample containers. Also some of these vendors use $1: 1$ reagent grade nitric acid to rinse sample containers. For the purposes of cleaning sample containers as out'ined in Section $B$ of this Attachment, both of these deviations from the definitions contained in Section $A$ of this procedure are permitted. 
Attachment B, Part B - RCRA Specific Comments Response 20

Method No.: ESP-900

Revision No.: 0

Date: August 27, 1988

Page: 8 of 20

MARTIN MARIETTA ENERGY SYSTEMS, INC.

ENVIRONMENTAL SURVEILLANCE PROCEDURES

D. Cleaning of sample containers that are recommended for use in the analysis of:

$\begin{array}{ll}\text { BOD } & \text { Extractable Organics } \\ \text { COD } & \text { Phenols } \\ 0 i 1 \text { and Grease } & \text { TOC }\end{array}$

1. Wash containers, closures, and teflon liners in hot tap water with laboratory grade non-phosphate detergent.

2. Rinse three times with tap water.

3. Rinse one time with $1: 1$ nitric acid.

4. Rinse three times with deionized water.

5. Air dry in contaminant-free environment.

6. Place liners in closures and place closures on containers.

II. Emergency Disposable Sample Container Cleaning

New jars may be used to collect samples in an emergency. These containers would also be acceptable on an emergency basis for the collection of water samples for extractable and pesticide organic analyses as well as metal analyses. These jars cannot be used for the collection of water samples for purgeable organic analyses.

The rubber sealing ring should not be in contact with the jar and aluminum foil should be used, if possible, between the jar and the sealing ring, If possible, the jar and aluminum foil should be rinsed with solvent 2 and allowed to air dry before use. Several empty bottles and lids should be submitted to the laboratory as blanks for quality control purposes.

2 In these circumstances pesticide grade solvents such as petroleum, ether, hexane, methanol or isopropyl alcohol may be used. 
Method No.: ESP-900

Revision No.: 0

Date: August 27, 1988

Page: 9 of 20

MARTIN MARIETTA ENERGY SYSTEMS, INC.

ENVIRONMENTAL SURVEILLANCE PROCEDURES

\section{ATTACHMENT 2}

I. Cleaning Procedures for Teflon or Glass Field Sampling Equipment Used for the Collection of Samples for Trace Organic Compounds and/or Metals Analyses $^{3}$

1. Equipment will be washed thoroughly with laboratory detergent and hot water using a brush $i 0$ remove any particulate matter or surface film.

2. The equipment will be rinsed thoroughly with hot tap water. 3. Rinse equipment with at least a 10 percent nitric acid
solution.

4. Rinse equipment thoroughly with tap water.

5. Rinse equipment thoroughly with deionized water.

6. Rinse equipment twice with solvent and allow to air dry for at least 24 .hours.

7. Wrap equipment with aluminum foil to prevent contamination during storage and/or transport to the field.

3 When this sampling equipment is used to collect samples that contain oil, grease or other hard to remove materials, it may be necessary to rinse the equipment several times with pesticide-grade acetone or hexane to remove the materials before proceeding with step 1 . In extreme cases, it may be necessary to steam clean the field equipment before proceeding with Step 1. If the field equipment cannot be cleaned utilizing these procedures, it should be discarded.

4 Small and awkward equipment such as vacuum bottle inserts and well bailers may be soaked in the nitric acid solution instead of being rinsed with it. Fresh nitric acid solution should be prepared for each cleaning session. 
Attachment B, Part B - RCRA Specitıc Comments Response 20

Method No.: ESP-900

Revision No.: 0

Date: August 27, 1988

Page: 10 of 20

MARTIN MARIETTA ENERGY SYSTEMS, INC.

ENVIRONMENTAL SURYEILLANCE PROCEDURES

8. Rinse the Teflon or glass sampling equipment thoroughly with tap water in the field as soon as possible after use.

II. Cleaning Procedures for Stainless Steel or Metal Sampling Equipment Used for the Collection of Samples for Trace Organic Compounds and/or Metals Analyses $^{5}$

1. Wash equipment thoroughly with 1aboratory detergent and hot water using a brush to remove any particulate matter or surface film.

2. Rinse equipment thoroughly with hot tap water.

3. Rinse equipment thoroughly with deionized water.

4. Rinse equipment twice with solvent and allow to air dry for at least 24 hours.

5. Wrap equipment wiih aluminum foil to prevent contamination during storage and/or transport to the field.

6. Rinse the stainless steel or metal sampling equipment thoroughly with tap water in the field as soon as possible after use.

5 When this sampling equipment is used to collect samples that contain oil, grease or other hard to remove materials, it may be necessary to rinse the equipment several times with pesticide grade acetone or hexane to remove the materials before proceeding with Step 1 . In extreme cases, when equipment is painted, badly rusted, or coated with materials that are difficult to remove, it may be necessary to steam clean, wire brush, or sandblast equipment before proceeding with Step 1. Any stainless steel sampling equipment that cannot be cleaned using these procedures should be discarded. 
Attachment B, Part B - RCRA Specific Comments Response 20

\author{
Method No.: ESP-900 \\ Revision No.: 0 \\ Date: August 27, 1988 \\ Page: 11 of 20
}

MARTIN MARIETTA ENERGY SYSTEMS, INC.

ENVIRONMENTAL SURVEILLANCE PROCEDURES

\title{
III. Cleaning Procedures for Automatic Wastewater Sampling Equipment
}

\section{A. General}

Automatic samples will be cleaned as follows:

1. The exterior and accessible interior (excluding the waterproof timing mecharism) portions of automatic samplers will be washed with laboratory cietergent and rinsed with tap water.

2. The face of the timing case mechanism will be cleaned with a clean damp cloth.

3. All tubing (sample intake and pump tubing) will be discarded after use.

4. New precleaned, silastic pump tubing (see Section IV. A.) will be installed.

5. When utilizing the samplers for collecting samples for metals and/or organic compounds analyses, the metal distributor tubes should not be used; only glass or silastic pump tubing should be used for this purpose.

B. Automatic Sampler Headers

1. Disassemble header and using a bottle brush, wash with hot water and phosphate free laboratory detergent.

2. Rinse thoroughly with deionized water.

3. Reassemble header, let dry thoroughly and wrap with aluminum foil. 
Attachment B, Part B - RCRA Specific Comments Response 20

Method No.: ESP-900

Revision No.: 0

Date: August 27, 1988

Page: 12 of 20

MARTIN MARIETTA ENERGY SYSTEMS, INC.

\section{ENVIRONMENTAL SURVEILLANCE PROCEDURES}

\section{Reusable Glass Composite Sample Containers ${ }^{6}$}

1. Wash containers thoroughiy with hot tap water and laboratory detergent, using a bottle brush to remove particulate matter and surface film.

2. Rinse containers thoroughly with hot tap water.

3. Rinse containers with at least 10 percent nitric acid.

4. Rinse containers thoroughly with tap water.

5. Rinse containers thoroughly with deionized water.

6. Rinse twice with solvent and allow to air dry for at least 24 hours.

7. Cap with aluminum foil or Tefion film.

8. After using, rinse with tap water in the field, seal with aluminum foil to keep the interior of the container wet, and return to the laboratory.

6 When these containers are used to collect samples that contain oil, greasi or other hard to remove materials, it may be necessary to rinse tile container. several times with pesticide grade acetone before proceeding with Step 1 . If these materials cannot be removed with acetone, the container should be discarded. Glass reusable composite containers used to collect samples at pesticide, herbicide, or other chemical manufacturing facilities that produce toxic or noxious compounds shall be disposed of "properly" (preferably at the facility) at the conclusion of sampling activities and shall not be returned for cleaning. Also, glass composite containers used to collect in-process wastewater samples at industrial facilities shall be discarded after sampling. Any bottles that have a visible film, scale, or discoloration remaining after this cleaning procedure shall also be discarded. 
Attachment B, Part B - RCRA Specific Comments Response 20

Method No.: ESP-900

Revision No.: 0

Dats: August 27, 1988

Page: 13 of 20

MARTIN MARIETTA ENERGY SYSTEMS, INC.

ENVIRONMENTAL SURVEILLANCE PROCEDURES

D. Plastic Reusable Composite Sample Containers ${ }^{7}$

1. Proceed with the cleaning procedures as outlined in III $C$ but omit Step 6.

E. Sequential Sample Bottles (Automatic Sampler Base for Sequential Mode)

1. Rinse with 10 percent nitric acid.

2. Rinse thoroughly with tap vater.

3. Wash using laboratory detergent, followed by tap and deionized water rinse.

4. Replace bottles in covered, automatic sampler base; cover with a) uminum foil for storage.

5. Rinse bottles in the field as soon as possible after using tap water.

F. Sequential Sample Bottles (Automatic Sampler Base for Sequential Mode) to be Used for Collecting Samples for Organic Compounds Analyses

1. Proceed as outlined in Steps 1-4 in Section III E.

2. Rinse twice with solvent and allow to air dry for at least 24 hours.

3. Replace in covered, automatic sampler base; cover with aluminum foil for sturage and mark the base as follows: "Cleaned for organic analyses."

7 Plastic reusable sample containers used to collect samples from facilities that produce toxic or noxious compounds or are used to collect in-process waste stream samples at industrial facilities will be disposed of properly (preferably at the facility) at the conclusion of the sampling artivities and will not be returned for cleaning. Any plastic composite sample containers that have a visible film, scale, or other discoloration remaining after this cleaning procedure will be discarded. 
Attachment B, Part B - RCRA Specific Comments

Response 20

Method No.: ESP-900

Revision No.: 0

Date: August 27, 1988

Page: 14 of 20

MARTIN MARIETTA ENERGY SYSTEMS, INC.

ENVIRONMENTAL SURVEILLANCE PROCEDURES

G. Bottle Siphons Used to Transfer Sample From Composite Container

1. Use a new siphon for each sampling location.

2. Use 3/8-inch Teflon tubing for samples collected for organic compounds analyses. The tubing should be rinsed with solvent and dried in the drying oven overnight before use. The ends of the siphon should be capped with aluminum foil and/or Teflon film for storage. The siphon should be flushed with sample thoroughly before use.

3. The 3/8-inch PVC tubing utilized for samples, other than those collected for organic compounds analyses, should be thoroughly flustied with sample before use.

H. Reusable Tefion Composite Mixer Rods

1. Follow procedure outlined in Section I:Steps 1-6.

2. Wrap rod in aluminum foil for storage.

IV. Cleaning Procedures for Sample Tubing

A. Silastic Rubber Pump Tubing Used in Automatic Samplers and Other Peristaltic Pumps

New cleaned tubing must be used for each automatic sampler set-up. The silastic rubber pump tubing need not be replaced in peristaltic pumps where the sample does not contact the tubing or where the pump is being used for purging purposes (i.e., not being used to collect samples).

The silastic tubing shall be cleaned as follows:

1. Flush tubing with hot tap water and phosphate-free laboratory detergent.

2. Rinse tubing thoroughly with hot tap water.

3. Rinse tubing with deionized water.

4. Install tubing in automatic sampler or peristaltic pump. 
Method No.: ESP-900

Revision No.: 0

Date: August 27, 1988

Page: 15 of 20

MARTIN MARIETTA ENERGY SYSTEMS, INC:

ENVIRONMENTAL SURVEILLANCE PROCEDURES

5. Cap both ends of tubing with aluminum foil.

B. Teflon Sample Tubing

Use only new Tefion tubing cleaned as follows for collection of samples for organic compounds analyses:

1. Teflon tubing may be precut in convenient lengths before cleaning to simplify handling.

2. rinse outside of tubing with solvent.

3. Flush interior of tubing with solvent.

4. Dry overnight in the drying oven.

5. Wrap tubing and cap ends with aluminum foil to prevent contamination during storage.

C. Polyvinyl chloride (PVC) Sample Tubing

1. Use only new tubing.

2. The tubing will be flushed with sample immediately before use to remove any residues from the manufacturing or extruding process.

3. Polyvinyl chloride tubing will be used selectively where organic compounds are not of concern.

4. Tubing should be stored in originil container and not removed from this container until needed.

D. Stainless Steel Tubing

1. Wash with laboratory detergent and hot water using a long, narrow, bottle brush.

2. Proceed with Steps 2-6 as outlined in Section II (footnote applies). 
Attachment B, Part B - RCRA Specific Comments Response 20

\author{
Method No.: ESP-900 \\ Revision No.: 0 \\ Date: August 27, 1988 \\ Page: 16 of 20
}

MARTIN MARIETTA ENERGY SYSTEMS, INC.

ENVIRONMENTAL SURVEILLANCE PROCEDURES

\title{
E. Glass Tubing
}

Use new glass tubing, precleaned as follows:

1. Rinse thoroughly with solvent.

2. Air dry for at least 24 hours.

3. Wrap tubing with aluminum foil to prevent contamination during storage.

4. Discard tubing after use.

V. Miscellaneous Equipment Cleaning Procedures

A. Well Sounders or Tapes Used to Measure Groundwater Levels ${ }^{8}$

1. Wash with laboratory detergent and tap water.

2. Rinse with tap water.

3. Rinse with deionized or organic-free water, as appropriate.

4. Equipment should be wrapped to prevent contamination during storage or transit.

B. Submersible Pumps and Hoses Used to Purge Groundwater We $175^{8}$

Proceed as outlined in Section V.A.

C. Portable Power Augers (Such as the Little Beaver)

1. The engine and power head should be cleaned with a power washer, steam jenny, or hand washed with a brush using detergent (does not have to be laboratory detergent but should not be a degreaser) to remove oil, grease, and hydrautic fluid from the exterior of the unit. These units should be rinsed thoroughly with tap water.

8 The same procedure appiies whether this equipment is cleaned in the washroom or in the field. 
Method No.: ESP-900

Revision No.: 0

Date: August 27, 1988

Page: 17 of 20

MARTIN MARIETTA ENERGY SYSTEMS, INC.

ENVIRONMENTAL SURVEILLANCE PROCEDURES

2. All auger flights and bits should be cleaned utilizing the procedures outlined in Section II (including footnote).

D. Miscellaneous Sampling and Flow Measuring Equipment

Miscellaneous flow measuring and sampling equipment shall be washed with laboratory detergent, rinsed with hot tap water, followed by a thorough deionized water rinse, and dried before being stored. This procedure is not used for any equipment utilized for the collection of samples for trace organic compounds or metals analyses.

E. Flow Meters, Field Analytical Equipment, and Other Field Instrumentation

The exterior of sealed, watertight equipment such as flow meters should be washed with a mild detergant (for example, liquid dishwashing detergent) and rinsed with tap water before storage. The interior of such equipment may be wiped with a damp cloth if necessary.

Other field instrumentation should be wiped with a clean, damp cloth; pH meter probes, conductivity probes, DO meter probes, etc. should be rinsed with deionized water before storage.

The desiccant in flow meters and other equipment should be checked and replaced if necessary each time the equipment is cleaned.

F. Ice Chests and Shipping Containers

All ice chests and reusable containers will be washed with laboratory detergent (interior and exterior) and rinsed with tap water and air dried before storage. In the event that an ice chest becomes severely contaminated, in the opinion of the field investigator, with concentrated waste or other toxic material, it shall be cleaned as thoroughly as possible, rendered unusable, and disposed of properly. 
Method No.: ESP-900

Revision No.: 0

Date: August 27, 1988

Page: 18 of 20

MARTIN MARIETTA ENERGY SYSTEMS, INC.

ENVIRONMENTAL SURVEILLANCE PROCEDURES

G. Pressure Field Filtration Apparatus 9 .

1. Proceed with steps 1 through 5 as outlined in Section I, assembling and applying pressure to the apparatus after each rinse step (water and acid) to drive rinse material through the porous glass filter holder in the bottom of the apparatus.

2. Assemble the apparatus and cap both the pressure inlet and sample discharge lines with aluminum foil to prevent contamination during storage.

H. Organic-Free Water Storage Containers

1. These containers will be used only for storing organic-free water.

2. New containers shall be prepared as outlined in Section III. C, Steps 1-5, then rinsed thoroughly with organic-free or Milli-Q water, filled with Milli-Q water and capped.

3. Used containers shall be capped with aluminum foil immediately after being used in the field.

4. The exterior of the container will be washed with laboratory detergent and rinsed with deionized water if necessary.

5. The interior of the container will be rinsed twice with solvent.

6. The interior of the container will be thoroughly rinsed with organic-free or Milli-Q water. The container will be filled with organic-free or Milli-Q water and capped with aluminum foil for storage.

9 The same procedure applies whether the pressure filtration apparatus is cleaned in the washroom or in the field. 
Method No.: ESP-900

Revision No.: 0

Date: August 27, 1988

Page: 19 of 20

MARTIN MARIETTA ENERGY SYSTEMS, INC.

ENVIRONMENTAL SURVEILLANCE PROCEDURES

\section{Field Equipment Cleaning Procedures}

\section{A. General}

Sufficient clean equipment should be transported to the field so that an entire study can be conducted without the need for field cleaning. However, this is not possible for some specialized items of field equipment (i.e., well drilling rigs, soil coring rigs, and other large pieces of field equipment). In addition, during particularly large-scale studies, it may not be practical or possible to transport to the field all of the cleaned field equipment required. The following procedures are to be utilized when equipment must be cleaned in the field.

B. Equipment Used for Environmental or Background Sample Collection Activities

For operations involving environmental or background samples, water quality sampling equipment (i.e., Kemmerers, buckets, Do dunkers, dredges, etc.) may be cleaned with ambient or deionized water between sampling locations. A brush may be used to remove deposits of material or sediment, if necessary. If deionized water is used, water samplers should be flushed with ambient water at the next sampling location before the sample is collected. It should be emphasized that these procedures cannot be used to clean equipment for the collection of samples for organic compounds or trace metals analyses.

Flow measuring equipment (i.e., weirs, staff gauges, velocity meters, and other stream gauging equipment) may be cleaned with tap water after use between measuring locations, if necessary.

C. Teflon, Stainless Steel or Metal Equipment Used to Collect Samples for Organic Compounds and Trace Metals Analyses

Note: Large soil boring or drill rigs should be cleaned as outlined in ESP-901 before boring or drilling operations.

1. Clean with tap water and laboratory detergent using a brush if necessary to remove particulate matter and surface films.

2. Rinse thoroughly with tap water. 
Attachment B, Part B - RCRA Specific Comments Response 20

Method No.: ESP-900

Revision No.: 0

Date: August 27, 1988

Page: 20 of 20

MARTIN MARIETTA ENERGY SYSTEMS, INC.

ENVIRONMENTAL SURVEILLANCE PROCEDURES

3. Rinse thoroughly with deionized or organic-free water.

4. Rinse twice with solvent.

5. Rinse thoroughly with organic-free water and allow to air dry as long as possible.

6. If organic-free water is not available, allow equipment to air dry as long as possible. Do not rinse with deionized or distilled water.

7. Wrap with aluminum foil (dull side in), if appropriate, to prevent contamination if equipment is going to be stored or transported. Large pieces of equipment (i.e., auger flights) may be wrapped in new Visqueen, or equivalent, for transport to the field. 


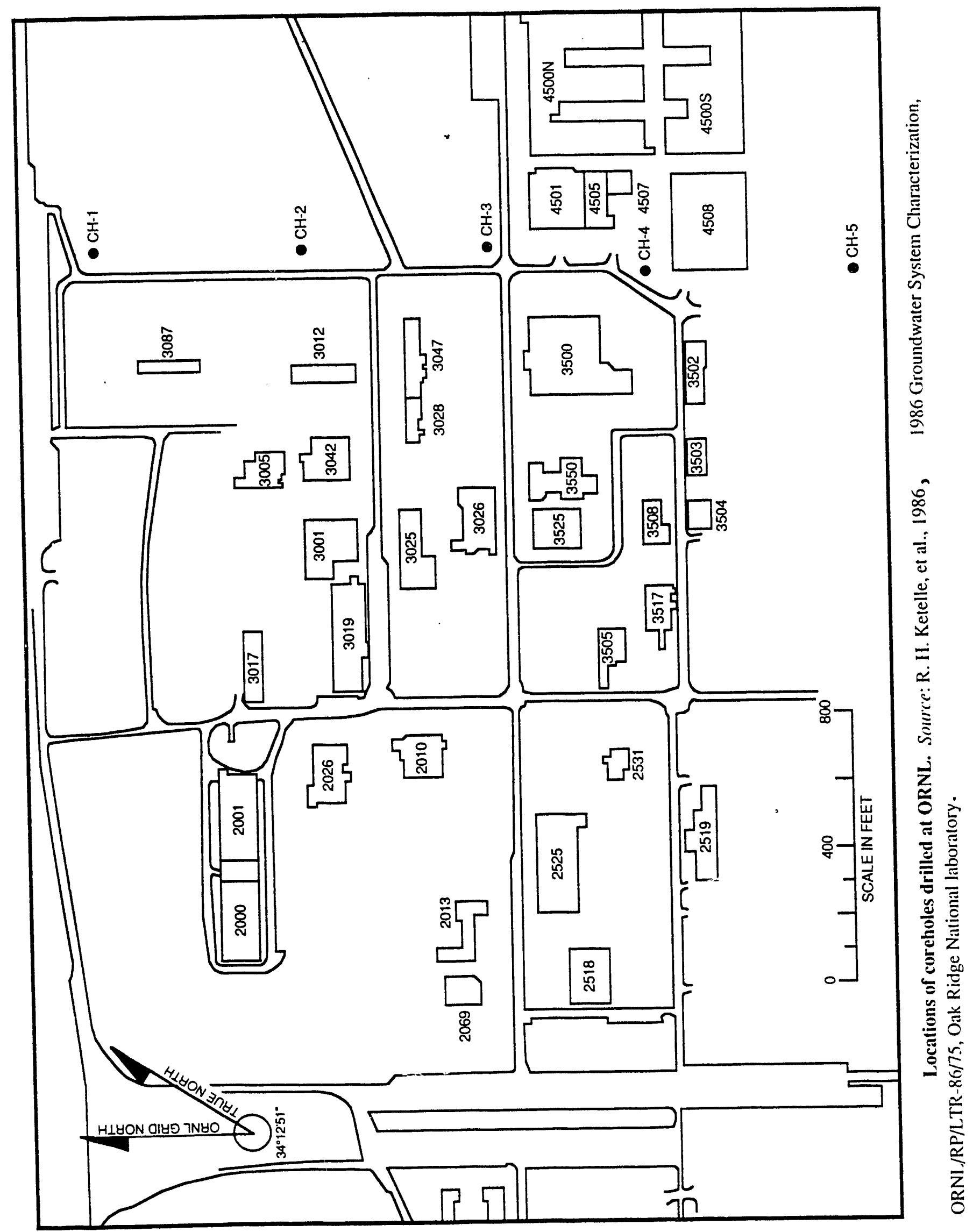


Attachment B, Part B - RCRA Specific Comments Response 33

Method Number: ESP 301-1

Revision Number: 0

Date: August 30, 1988

Page: 1 of 7

MARTIN MARIETTA ENERGY SYSTEMS, INC.

ENVIRONMENTAL SURVEILLANCE PROCEDURES

\section{SUBJECT: WATER SAMPLING USING A DIPPER}

\section{Scope and Application:}

This procedure describes the standard method and equipment for sampling of surface waters using a dipper. The dipper is useful in filling a sample bottle without contaminating the outside of the bottle. This makes it possible for the worker to avoid physical contact with the waste stream.

A pond sampler, or extended dipper allows sampling of streams, ponds, waste pits and lagoons as much as 15 feet from the bank or other secure footing for the sampling technician.

The samples may be used to obtain physical, chemical or radiological data. The resulting data may be qualitative or quantitative in nature and is appropriate for use in preliminary surveys as well as confirmatory sampling.

II. References:

A. A Compendium of Superfund Field Operations Methods, EPA/540/P$87 / 001$, U.S. Environmental Protection Agency, Washington, D.C., 1987.

B. Data Quality Objectives for Remedial Activities-Development Process, EPA/540/G-87/003, U.S. Environmental Protection Agency, Washington, D.C., 1987.

C. The Environmantal Survey Manual, DOE/EH-0053, Appendix E, Field Sampling Pro ocjls and Guidance, U.S. Department of Energy, 1987.

D. Engineering Support Branch Standard Operating Procedures and Quality Assurance Manual, U.S. Environmental Protection Agency, Athens, GA, 1986. 
Attachment B, Part B - RCRA Specific Comment Response 33

Method Number: ESP 301-1

Revision Number: 0

Date: August 30, 1988

Page: 2 of 7

MARTIN MARIETTA ENERGY SYSTEMS, INC.

ENVIRONMENTAL SURVEILLANCE PROCEDURES

E. Characterization of Hazardous Waste Sites - A Methods Manual: Volume II - Available Sampling Methods, 2nd Edition, EPA-600/4-84-076, U.j. Environmental Protection Agency, December, 1984.

III. Summary of Method:

The dipper is slowly dipped into the surface water and lifted out while avoiding splashing or otherwise disturbing the surface water. The sample is then carefully poured into a sample bottle.

IV. Comments:

This method can be used for most water sampling. When volatile organic analysis will be performed, extreme care must be taken to avoid disturbing or aerating the sample. Also, this method should not be used when a significant amount of material might remain on the dipper when pouring into the sample bottle (Such as heavy oil on the water surface).

V. Required Equipment and Apparatus:

A. Dipper - Container with handle; constructed of stainless steel, glass, teflon or other chemically inert material.

B. Pond Sampler - Also known as an extended dipper. The pond sampler may be assembled from the following equipment (See Figure 1):

1. Dipper: Container, cup or beaker similar to (A) above.

2. Telescoping Handle: Telescoping aluminum pole, 8 to 15 feet in length, with bolt holes (available at most hardware stores).

3. Clamp: "Varigrip" style clamp with bolt holes and bolts for attachment to telesccping pole (usually available from lab supply stores). 
Attachment B, Part B - RCRA Specific Comments Response 33

Method Number: ESP 301-1

Revision Number: 0

Date: August 30, 1988

Page: 3 of 7

MARTIN MARIETTA ENERGY SYSTEMS, INC.

ENVIRONMENTAL SURVEILLANCE PROCEDURES

VI. Safety:

It is Energy Systems policy to maintain an effective program for control of employee exposure to chemical, radiological, and physical stress which is consistent with the requirements of Martin Marietta Corporation, DOE, and OSHA established standards and requirements. All field personnel will be provided with appropriate protective clothing and safety equipment.

As a minimum, field personnel are required to wear steel capped safety shoes and safety glasses while performing this procedure. Refer to a site specific health-and safety plan for detailed health and safety procedures. This plan should be reviewed prior to beginning work.

VII. Procedures:

A. Use plastic sheet as ground cover for staging of equipment and/or materials as necessary to prevent equipment from coming in contact with potentially contaminated surfaces.

B. Select appropriate sample bottles (see ESP-701) and place them ready for use.

C. Submerge a clean dipper (or pond sampler) slowly into the water to avoid splashing or mixing.

D. Slowly lift the dipper from the water surface. Unless specified in the sampling plan, avoid floating materials.

E. Remove the cap from the sample bottle, and tilt the bottle slightly.

F. Pour the sample slowly from the dip'er down the inside of the sample bottle. Avoid splashing of the saipla. Assure that any suspended matter in the sample is transferred quantitatively to the sample bottle.

G. Leave adequate air space in the bottle to allow for expansion, except for VOA vials.

H. Label the bottle carefully, and clearly in accordance with ESP-500. Enter all information accurately, and check to be sure it is legible. 
Attachment B, Part B - RCRA Specific Comments Response 33

Method Number: ESP 301-1

Revision Number: 0

Date: August 30, 1988

Page: 4 of 7

MARTIN MARIETTA ENERGY SYSTEMS, INC.

ENVIRONMENTAL SURVEILLANCE PROCEDURES

I. Samples will be placed in containers defined according to the needs, and then, when appropriate, packed with ice in coolers as soon as practical. Packaging, labeling, and preparation for shipment will follow procedures as specified in ESP-800.

J. Complete field logbook and chain-of-custody forms in accordance with ESP -400 and ESP-500.

\section{Contamination Control}

Sampling tools and equipment will be protected from sources of contamination prior to sampling and decontaminated prior to and between sampling as specified in ESP-900. Liquids and materials from decontamination operations will be handled in accordance with ESP-1000. Sample containers will also be protected from sources of contamination. Sampling personnel shall wear chemical resistant gloves when handling the trier and soil samples. Gloves will be decontaminated or disposed of between samples.

IX. QA/OC

In addition to adhering to the specific requirements of this sampling protocol and any supplementary site specific procedures, the minimum $Q A \backslash Q C$ requirements for this sampling activity are the following.

\section{A. Control of Deviations}

When feasible, any departure from specified requirements will be justified and authorized prior to deviating from the requirements. Deviations shall be sufficiently documented to allow repetition of the activity as actually performed.

\section{B. OC Samples}

The number and types of $Q C$ samples including duplicate samples, field blanks, equipment blanks and trip blanks will be collected or prepared as specified in the governing plans and procedures. 
Attachment B, Part B - RCRA Specific Comments Response 33

Method Number: ESP 301-1

Revision Number: 0

Date: August 30,1988

Page: 5 of 7

MARTIN MARIETTA ENERGY SYSTEMS, INC.

ENVIRONMENTAL SURVEILLANCE PROCEDURES

\section{Verification}

Verification activities are required for the above practices including surveillance and periodic record audits. These activities will be documented and become part of the completed project records.

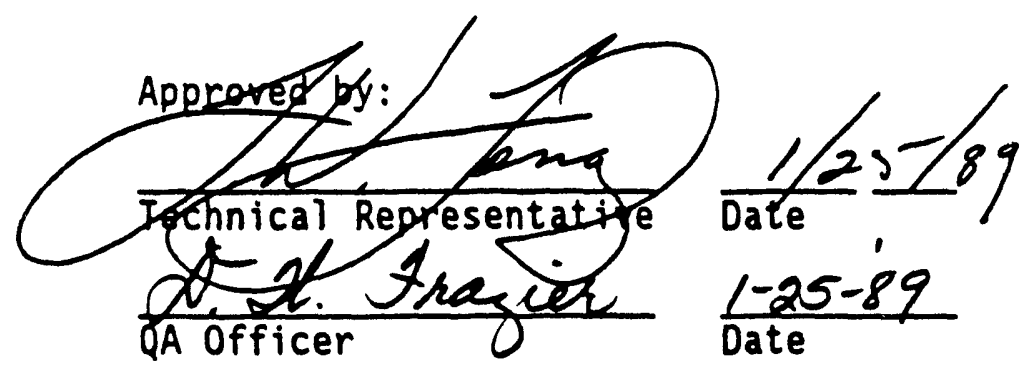


Attachment B, Part B - RCRA Specific Comments

Response 33

Method Number: ESP 301-1

Revision Number: 0

Date: August 30, 1988

Page: 6 of 7

MARTIN MARIETTA ENERGY SYSTEMS, INC.

ENVIRONMENTAL SURVEILLANCE PROCEDURES

\section{Attachment 1}

SUJBECT: WATER SAMPLING USING A DIPPER

Field Checklist

Dipper *

Pond Sampler, if appropriate

Logbook

Sample Bottles w/Lids

Safety Equipment, including steel toe shoes, gloves, and eye protection.

Ice/Cooler, as required

Health \& Safety Plan

Sampling and Analysis Plan

Appropriate Containers for Waste and Equipment.
Black Indelible Pen

Labels

Lab Wipes

Decontamination

Equipment**

Chain-of-Custody

forms

Custody seals, as required

Chemical Preservatives, as required

Plastic sheet

* Provide sufficient quantities to minimize field decontamination activities.

* See Section ESP-900 for decontamination of sampling devices. 
Attachment B, Part B - RCRA Specific Comments Response 33

Method Number: ESP 301-1

Revision Number: 0

Date: August 30, 1988

Page: 7 of 7

MARTIN MARIETTA ENERGY SYSTEMS, INC.

ENVIRONMENTAL SURVEILLANCE PROCEDURES
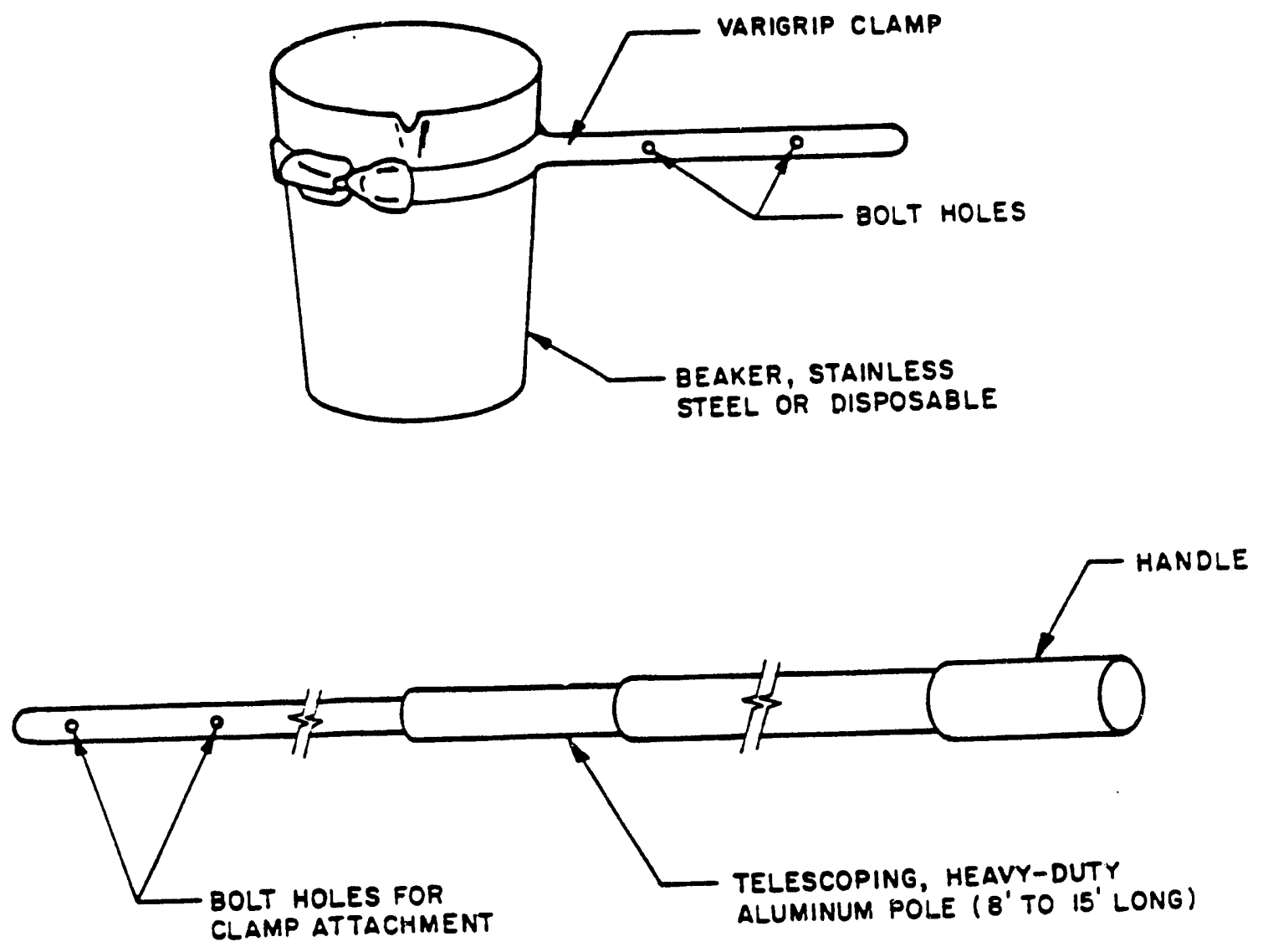

Flgure 1. Pond Sampler 
Attachment B, Part B - RCRA Specific Comments Response 34

Method Number: ESP-303-4

Revision No. : 0

Date: August 24, 1988

Page: 1 of 7

MARTIN MARIETTA ENERGY SYSTEMS, INC.

ENVIRONMENTAL SURVEILLANCE PROCEDURES

\section{SUBJECT: PENETRATION TEST AND SPLIT-BARREL SAMPLING}

\section{Scope and Application}

This procedure describes the collection of samples using a split-barrel sampler and the procedure to measure resistance to the penetration of the sampler.

II. References:

A. ASTM Standard Method D 1586-84, "Penetration Test and Split-Barrel Sampling of Soils".

B. A Compendium of Superfund Field Operations Methods, D.C., 1987 .

C. Data Quality Objectives for Remedial Response Activities, EPA/540/G$87 / 003$, U.S. Environmental Protection Agency, Washington, D.C., 1987.

D. Engineering Support Branch Standard Operating Procedures and Quality Assurance Manual, U.S. Environmental Protection Agency, Athens, GA, 1986.

\section{Summary of Method}

Drilling equipment is used to advance a borehole. A split-barrel sampler is attached to flush-joint steel drill rods and is driven into the bottom of the open borehole using a hammer and anvil. If samples are collected for chemical analysis, the -plit-barrel sampler will be made of $304 / 316$ stainless steel. The numser of hammer blows required to drive the sampler into the soil is recorded as a measure of resistance. The sampler then is retrieved and opened. The sample length and a description of the sample material is recorded in a logbook as describe in the sampling and analysis plan. An aliquot of the sample material is containerized.

\section{Comments}

This method provides best resuits in consolidated soils or competent material. 
Attachment B, Part B - RCRA Specific Comments Response 34

Method Number: ESP-303-4

Revision No. : 0

Date: August 24, 1988

Page: 2 of 7

\author{
MARTIN MARIETTA ENERGY SYSTEMS, INC.
}

ENVIRONMENTAL SURVEILLANCE PROCEDURES

Sampling for geotechnical analysis will follow reference A. Sampling for chemical analysis will require attention to cleaning and use of stainless steel samplers.

\title{
V. Required Equipment and Apparatus
}

A. Drilling Equipment - Any drilling equipment capable of providing a borehole at the required depth. Experience has shown the following types of equipment to be suitable:

o Hollow-stem flight augers.

- Drag, chopping, and fishtail bits used in conjunction with rotary or casing-advance drills.

- Roller-cone bits used in conjunction with rotary or casingadvance drills.

- Solid, bucket, and hand augers.

B. Sampling Rods - Flush-joint steel drill rods.

C. Split-Barrel Sampler - A split-barrel sampler constructed with the dimensions indicated in Figure 1. The sampler will have a hardened steel shoe. The sampler may be 1 ined. If samples are to be taken for chemical analysis, use $304 / 326$ stainless steel.

D. Drive-Weight Assembly

1. Hammer and Anvil - The hammer will be a solid, rigid, metallic mass weighing $140 \pm 21 \mathrm{~b}$ (for geotechnical samples) or $3001 \mathrm{~b}$. if conditions warrant. The hammer falls along a guide and strikes the anvil, making contact.

2. Hammer Drop System - Rope-csthaad, trip, semi-automatic, or automatic hammer drop systems may be used, providing the lifting apparatus will not cause sampler penetration while reengaging and lifting the hammer.

E. Sample catcher as specified in the sampling and analysis plan. 
Attachment B, Part B - RCRA Specific Comments Response 34

Method Number: ESP-303-4

Revision No. : 0

Date: August 24, 1988

Page: 3 of 7

MARTIN MARIETTA ENERGY SYSTEMS, INC.

ENVIRONMENTAL SURVEILLANCE PROCEDURES

\section{Safety}

It is Energy Systems policy to maintain an effective program for control of employee exposure to chemical, radiological, and physical stress which is consistent with the requirements of Martin Marietta Corporation, DOE, and OSHA established standards and requirements. All field personnel will be provided with appropriate protective clothing and safety equipment.

As a minimum, field personnel are required to wear steel capped safety shoes, hard hats, and safety glasses while performing this procedure. Refer to a site specific health and safety plan for detailed health and safety procedures. This plan should be reviewed prior to beginning work.

\section{Procedures}

A. Advance the borehole to the depth specified in the sampling and analysis plan.

B. Remove excess cuttings from the borehole.

C. Attach the sampier to the sampling rods and lower into the borehole. Do not allow the sampler to drop onto the soil or material to be sampled or penetrate the soil or material to any degree.

D. Position the hammer and anvil above the sampling rods.

E. Rest the dead weight of the sampler, sampling rod, hammer and anvil assembly on the bottom of the borehole.

F. Apply a series of seating blows.

G. Mark the drill rods in successive 6 -inch increments.

H. Drive the sampler into the soil with hammer blows. Apply the hammer blows by using one of the following methods: 
Attachment B, Part B - RCRA Specific Comments Response 34

Method Number: ESP-303-4

Revision No. : 0

Date: August 24, 1988

Page: 4 of 7

MARTIN MARIETTA ENERGY SYSTEMS, INC.

ENVIRONMENTAL SURVEILLANCE PROCEDURES

1. A trip, automatic, or semi-automatic hammer drop system which lifts the hammer and allows it to drop 30 inches unimpeded. If geotechnical samples are taken, allow a 30 inch drop.

2. A cathead to pull a rope attached to the hammer, provided the cathead is free of rust, 011 , or grease and has a 6 to 10 inch diameter.

I. Count the number of blows applied to each 6 -inch drill rod increment until one of the following occurs:

1. A total of 50 blows are applied in a single 6 -inch increment.

2. A total of 100 blows are applied.

3. 10 successive blows result in no advancement of the sampler.

4. The sampler is advanced 18 inches.

For other penetration tests different penetration criteria will be used.

J. Retrieve and open the sampler. Avoid contact of the sample with the hands of sampling personnel or with tools.

K. Observe and record the soil sample lithology or description of the sample material.

H. Samples will be placed in containers defined according to the analytical needs, then, when appropriate, packed with ice in coolers as soon as practical. Packaging, labeling, and prepiration for shipment will follow procedures as specified in ESP-800.

1. Complete field logbook and chain-of-custody forms in accordance with ESP-400 and ESP-500. Enter sample information in the logbook.

\section{Contamination Control}

Sampling tools and equipment will be protected from sources of contamination prior to sampling and decontaminated prior to and between sampling as specified in ESP-900. Liquids and materials from decontamination operations will be handled in accordance with ESP-1000. Sample containers will also be protected from sources of contamination. 
Attachment B, Part B - RCRA Specific Comments Response 34

Method Number: ESP-303-4

Revision No. : 0

Date: August 24, 1988

Page: 5 of 7

MARTIN MARIETTA ENERGY SYSTEMS, INC.

ENVIRONMENTAL SURVEILLANCE PROCEDURES

Sampling personnel will wear chemical resistant gloves when handling the sampling equipment and samples. Gloves will be decontaminated or disposed between samples.

IX. $Q A / O C$

In addition to adhering to the specific requirements of this sampling protocol and any supplementary site specific procedures, the minimum $Q A / Q C$ requirements for the this sampling activity are the following:

A. Control of Deviations

When feasible, any departure from specified requirements will be justified and authorized prior to deviating from the requirements. Deviation shall be sufficiently documented to all repetition of the activity as actually performed.

B. QC Samples

The number and types of $Q C$ samples including duplicate samples, field blanks, equipment blanks and trip blanks will be collected or prepared as specified in the governing plans and procedures.

C. Verification

Verification activities are required for the above practices including surveillance and periodic record audits. These activities will be documented and become part of the completed project records.
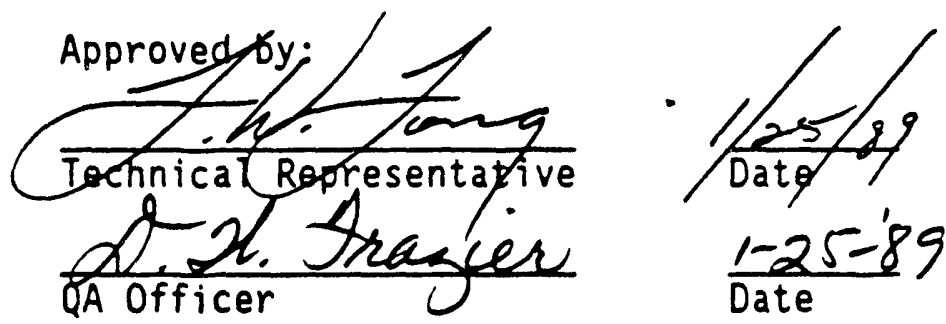
Attachment B, Part B - RCRA Specific Comments Response 34

Method Number: ESP-303-4

Revision No. : 0

Date: August 24, 1988

Page: 6 of 7

MARTIN MARIETTA ENERGY SYSTEMS, INC.

ENVIRONMENTAL SURVEILLANCE PROCEDURES

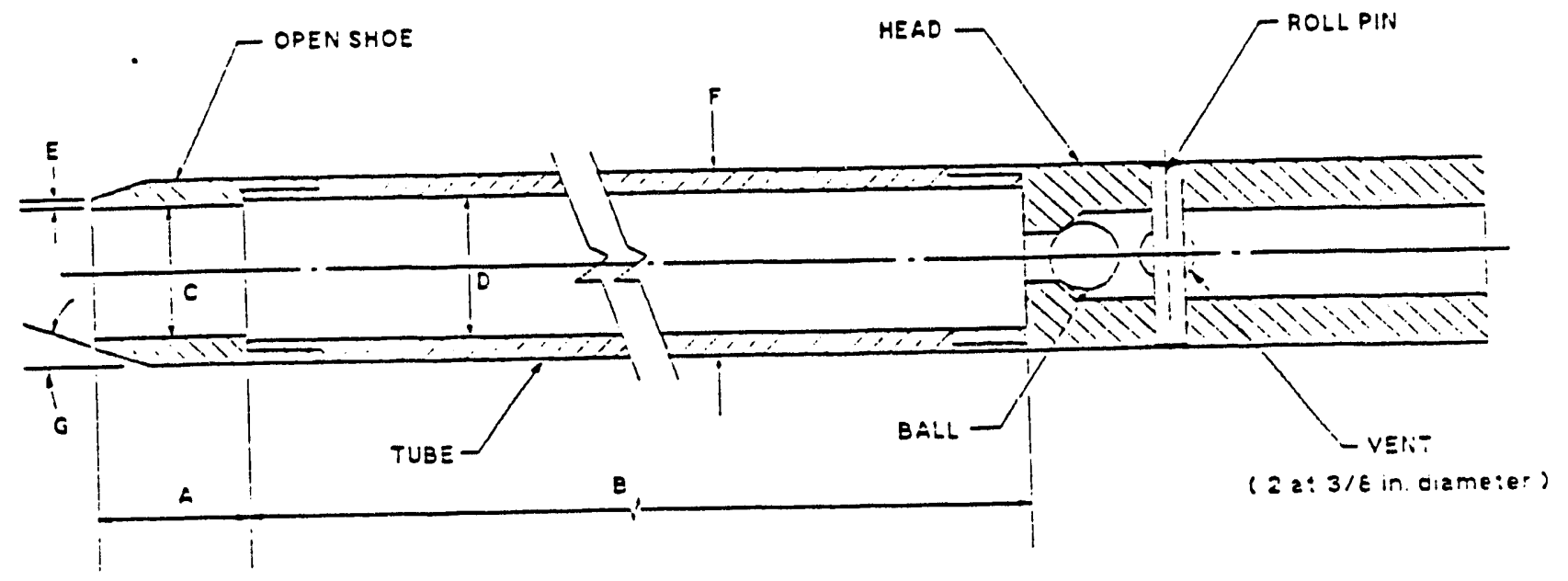

a. $1.0102 .0 \mathrm{ln}$

B. $18.01030 .0 \mathrm{ln}$.

c. $1.375: 0.005 \mathrm{ln}$.

$0 \cdot 9.50 \cdot 0.0 \leq \cdot 0.00 \mathrm{in}$

E. $0.90: 0.02 \mathrm{in}$

F $\cdot 2.00 \cdot 0.05 \cdot 0.00 \mathrm{in}$

$G \cdot 16.0: 023.0$ oegrees

Figure 1. Typical Split Barrel Sampler 
Attachment B, Part B - RCRA Specific Comments Response 34

Method Number: ESP-303-4

Revision No. : 0

Date: August 24, 1988

Page: 7 of 7

MARTIN MARIETTA ENERGY SYSTEMS, INC.

ENVIRONMENTAL SURVEILLANCE PROCEDURES

\section{ATTACHMENT I}

SUBJECT: PENETRATION TEST AND SPLIT-BARREL SAMPLING

$X$. Field Checklist

Spl it Barrel Sampler*

Logbook

Sample Containers

with lids

Safety Shoes

Gloves

Safety Glasses or Monogoggles

Hard Hats

Ice/Cooler, as required

Custody Seals or

Evidence Tape

Chain-of-Custody Forms
Black Indelible

Ink

Labels

Sampling and

Analysis Plan

Health and Safety Plan

Appropriate Containers for Waste and Waste Equipment

Decontamination Equipment**

Lab Wipes

Plastic Sheet

* Acquire sufficient quantity to minimize field decontamination requirements.

* See Section ESP-900 for decontamination sampling devices and ESP-901 for decontamination of heavy equipment. 
Attachment B, Part B - RCRA Specific Comments Response 34

Method No.: ESP $303-6$

Revision No.: 0

Date: August 31, 1988

Page: 1 of 10

MARTIN MARIETTA ENERGY SYSTEMS, INC.

ENVIRONMENTAL SURVEILLANCE PROCEDURES

SUBJECT: ROCK CORING AND SAMPLE COLLECTION

I. Scope and Application

This procedure describes methods to obtain and describe relatively undisturbed subsurface rock samples.

11. References

A. RCRA Ground-Water Monitoring Technical Enforcement Guidance Document, U.S. Environmenta1 Protection Agency, 1986.

B. Engineering Support Branch Standard Operating Procedures and Quality Assurance Manual, U.S. Environmental Protection Agency, Athens, GA, 1986.

C. A Compendium of Superfund Field Operation Methods, EPA/540/P-87/001, U.S. Environmental Protection Agency, Washington, D.C., 1987.

D. "Technical Memorandum No. 3-347," Waterways Equipment Station, Vicksburg, Mississippi, 1953.

E. "Sample Examination Manual," AAPG Methods in Exploration Series, 1981.

F. "Rock Color Chart," Geological Society of America, Bould̦er, Colorado, 1948.

G. "Diamond Core Drilling for Site Investigations," ASTM D2113, 1983.

\section{I:I. Summary of Method}

Rock coring is accomplished using different methods such as direct rotary drilling, where the borehole is drilled by rotating a bit and cuttings are removed by continuous circulation of a drilling fluid as the bit penetrates the formation and side-wall coring, which involves taking cores perpendicular to the borehole with specialized wireline equipment. core samples of the rock are collected and described. 
Attachment B, Part B - RCRA Specific Comments Response 34

Method No.: ESP 303-6

Revision No.: 0

Date: August 31, 1988

Page: 2 of 10

\author{
MARTIN MARIETTA ENERGY SYSTEMS, INC.
}

ENVIRONMENTAL SURVEILLANCE PROCEDURES

\title{
IV. Comments
}

The circulation fluid will be as specified in the sampling and analysis plan and would normally include air, potable water and drilling fluids. The description and classification of rock core samples will be accomplished only by geologists trained and experienced in the methods specified below. Sampling equipment used to take samples for chemical analysis will be cleaned and decontaminated prior to use.

V. Required Equipment and Apparatus

A. Drill rig

B. Core barrels

C. Longitudinally-split innertubes

D. Core bits

E. Reaming shells

F. Core lifters

G. Casings

H. Orit1 rods

I. Auxiliary equipment as required

J. Core boxes

VI. Safety

It is Energy Systems policy to maintain an effective program for control of employee exposure to chemical, radiological, and physical stress which is consistent with the requirements of Martin Marietta Corporation, DOE, and OSHA established standards and requirements. All field personnel will be provided with appropriate protective clothing and safety equipment.

As a $m$ ni num, field personnel are required :o wear steel capped safety shoes, nard hats, and safety glasses while performing this procedure. Refer to a site specific health and safety plan for detailed health and safety procedures. This plan should be reviewed prior to beginning work.

VII. Procedures

A. The borehole is advanced to the depth specified in the sampling and analysis plan and a rock core is obtained. 
Method No.: ESP 303-6

Revision No.: 0

Date: August 31, 1988

Page: 3 of 10

\section{MARTIN MARIETTA ENERGY SYSTEMS, INC.}

\section{ENVIRONMENTAL SURVEILLANCE PROCEDURES}

B. The site geologist will monitor core drilling efforts and take custody of the core when it is released from the coring device at the surface.

C. The site geologist will also identify and locate the drilling sites; determine the diameter of the borehole, depth coring begins and ends and total depth of the well; and provide an overview and supervision of the coring.

D. If a core is found that exceeds the permissible radiation levels established in the health and safety plan, the work will be halted and the situation evaluated. The following steps will be taken:

1. The hazardous work permit (HWP) will be upgraded and Energy Systems will be notified by the ES\&H Manager.

2. Personal protective equipment will be upgraded to the proper HWP.

3. Work will be resumed at the direction of the ES\&H Manager or designee.

4. The core will be labeled as radioactive and placed in a separate box.

5. The core will be disposed of as radioactive waste.

E. The site geologist logs the core and records pertinent information. The following information is recorded as a minimum:

Corehole number

Project ium.ier and name

Location

Geologist or engineer

Ground elevation

Drilling contractor

Casing type and diameter

Type of drilling fluid, if used

Rig reactions (chatter, etc.)

Depth to water encountered

Top and bottom of cored interval 
Method No.: ESP $303-6$

Revision No.: 0

Date: August 31, 1988

Page: 4 of 10

MARTIN MARIETTA ENERGY SYSTEMS, INC.

ENVIRONMENTAL SURVEILLANCE PROCEDURES

- Fluid gain or loss

0 Depth and percentage of fluid losses

- Core run length

- Coring rate in minutes per foot

o Percentage of recovery

- Core breakage due to discontinuities (natural fractures vs coring induced breaks)

- Total core breakage

- Number of breaks per foot

- Rock classification and lithology

$0 \quad$ Lithologic changes and boundaries

F. The site geologist should attempt to classify the rock core as recommended below.

1. Lithology and Texture: Lithologic description of the rock and its mineral composition (the geologic name, such as granite, basalt, or sanjstone, usually describes the rock's origin). Description of rock matrix including grain size, sorting, packing, cementation, etc. (i.e., interlocking, cemented, or laminated-foliated).

2. Color: The color descriptions conform to the Geological Society of America (GSA) Rock Color Chart (Ref. 6). Numerical Munsell Notation is acceptable. The major color is listed first with any accessory colors thereafter (e.g., clay, yellowbrown with occasional light-green mottles). If secondary or tertiary descriptions are used, the color designation follows each descriptor.

3. Hardness: Hardness is described using Mohs' scale of hartiess.

4. Weathering: Terms used to describe weathering are described below.

Descriptive Term

Fresh

\section{Defining Characteristics}

Rock is unstained. May be fractured, but discontinuities are not stained. 
Attachment B, Part B - RCRA Specific Comments

Response 34

Method No.: ESP 303-6

Revision No.: 0

Date: August 31, 1988

Page: 5 of 10

MARTIN MARIETTA ENERGY SYSTEMS, INC.

ENVIRONMENTAL SURVEILLANCE PROCEDURES

Slightly

Rock is unstained. Discontinuities shown some staining on the surfaces of rocks, but discoloration does not penetrate rock mass.

Moderate

Discontinuity surfaces are stained. Discoloration may extend into rock along discontinuity surfaces.

High

Individual rock fragments are thoroughly stained and can be crushed with pressure hammer. Discontinuity surfaces are thoroughly stained and may be crumbly.

Severe

Rock appears to consist of gravelsized fragments in a "soil" matrix. Individual fragments are thoroughly discolored and can be broken with fingers.

5. Grain Size: Term that describes fabric as fine-grained, medium-grained, or coarse-grained. The modified Wentworth scale is normally used.

6. Description of Bedding or of Joint or Fracture Spacing: Description should be according to the following:

Spacing

$<2$ in.

2 in. to $1 \mathrm{ft}$

$1 \mathrm{ft}$ to $3 \mathrm{ft}$

$3 \mathrm{ft}$ to $10 \mathrm{ft}$

$>10 \mathrm{ft}$

(after Deere, 1963)
Bedding or

Joints

Very close

Close

Moderately close

Wide

Very wide
Foliation

Very thin

Thin

Medium

Thick

Very thick

7. Discontinuity Descriptions: Terms that describe number, depth, and type of natural discontinuities. Also described are density, orientation, staining, planarity, alteration, joint or fracture fillings, and structural features. 
Method No.: ESP 303-6

Revision No.: : 0

Date: August 31, 1988

Page: 6 of 10

MARTIN MARIETTA ENERGY SYSTEMS, INC.

ENVIRONMENTAL SURVEILLANCE PROCEDURES

8. Stratigraphic Unit: Term used to assign local geological name, if appropriate, and to identify stratigraphic equivalents, if applicable.

G. The core logs should also include a complete visual description of the mineralogy of the rock, of any tests run in the bore hole, and/ or placement and construction details of piezometers, wells, and other rig monitoring devices.

H. The rock core is marked immediately after being extruded from the core barrel with reference lines and depths using permanent felt tip markers. It is marked with two lines along the long axis of the core, black line always on the right and red line on the left when the top of the core is away from you. The core is fitted together and measured. Any unrecovered interval is understood to be at the bottom unless otherwise determined.

1. Core samples must be placed into core boxes in the sequence of recovery, with the top of the core placed in the upper left hand corner of the box. At the bottom of each core run, spacer blocks must be placed to separate the runs. The spacer is indelibly labeled with the drilling depth to the bottom of the core run, regardless of how much core was actually recovered from the run.

J. Spacer blocks are also placed in the core box and labeled appropriately to indicate zones of core loss, if known. Where core samples are removed for laboratory testing, blocks equal in length to the core removed are placed in the box. If wooden core boxes are used, spacer blocks are nailed securely in place.

K. The core boxes for each boring are consecutive $y$ numbered from the top of the boring to the bottom. Core from onls o.le boring is to be placed in a core box.

L. Photograph records as specified in the sampling and analys is plan of core boxes are an essential part of the core logging and handling procedure and should be done whenever rock core is recovered.

M. When transporting boxed core, the box is moved only if the lid is closed and secured with tape or nails.

N. Packaging, labeling, and preparation for shipment will follow procedures as specified in ESP- 800 . 
Attachment B, Part B - RCRA Specific Comments Response 34

Method No.: ESP 303-6

Revision No.: : 0

Date: August 31, 1988

Page: 7 of 10

MARTIN MARIETTA ENERGY SYSTEMS, INC.

ENVIRONMENTAL SURVEILLANCE PROCEDURES

0. Complete field logbook and chain-of-custody forms in accordance with ESP-400 and ESP-500. Enter sample information in the logbook.

\section{Contamination Control}

Sampling tools and equipment will be protected from sources of contamination prior to sampling and decontaminated prior to and between sampling as specified in ESP-900. Liquids and materials from decontamination operations will be handled in accordance with ESP -1000 . Sample containers will also be protected from sources of contamination. Sampling personnel shall wear chemical resistant gloves when handling the sampling equipment and samples. Gloves will be decontaminated or disposed of between samples.

IX. $Q A / Q C$

In addition to adhering to the specific requirements of this sampling protocol and any supplementary site specific procedures, the minimum $Q A / Q C$ requirements for this sampling activity are the following.

\section{A. Control of Deviations}

When feasible, any departure from specified requirements will be justified and authorized prior to deviating from the requirements. Deviation shall be sufficiently documented to allow repetition of the activity as actually performed.

B. OC Samples

The number and types of $Q C$ samples including duplicate samples, field blanks, equipment blanks and trip blanks will be coliected or prepared as specified in the governing plans and procedure!. . 
Attachment B, Part B - RCRA Specific Comments Response 34

Method No.: ESP $303-6$

Revision No.: : 0

Date: August 31, 1988

Page: 8 of 10

\section{MARTIN MARIETTA ENERGY SYSTEMS, INC.}

\section{ENVIRONMENTAL SURVEILLANCE PROCEDURES}

\section{Verification}

Verification activities are required for the above practices including surveillance and periodic record audits. These activities will be documented and become part of the completed project records.

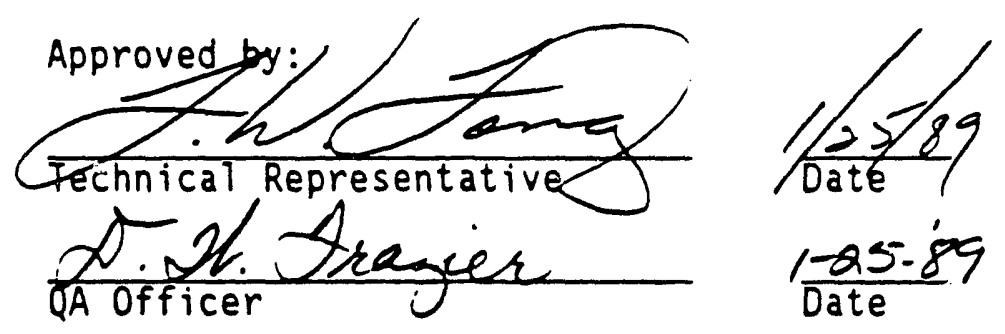


Attachment B, Part B - RCRA Specific Comments Response 34

Method No.: ESP 303-6

Revision No.: 0

Date: August 31, 1988

Page: 9 of 10

MARTIN MARIETTA ENERGY SYSTEMS, INC.

ENVIRONMENTAL SURVEILLANCE PROCEDURES

\section{ATTACHMENT I}

SUBJECT: ROCK CORING AND SAMPLE COLLECTION

$X$. Field Checklist

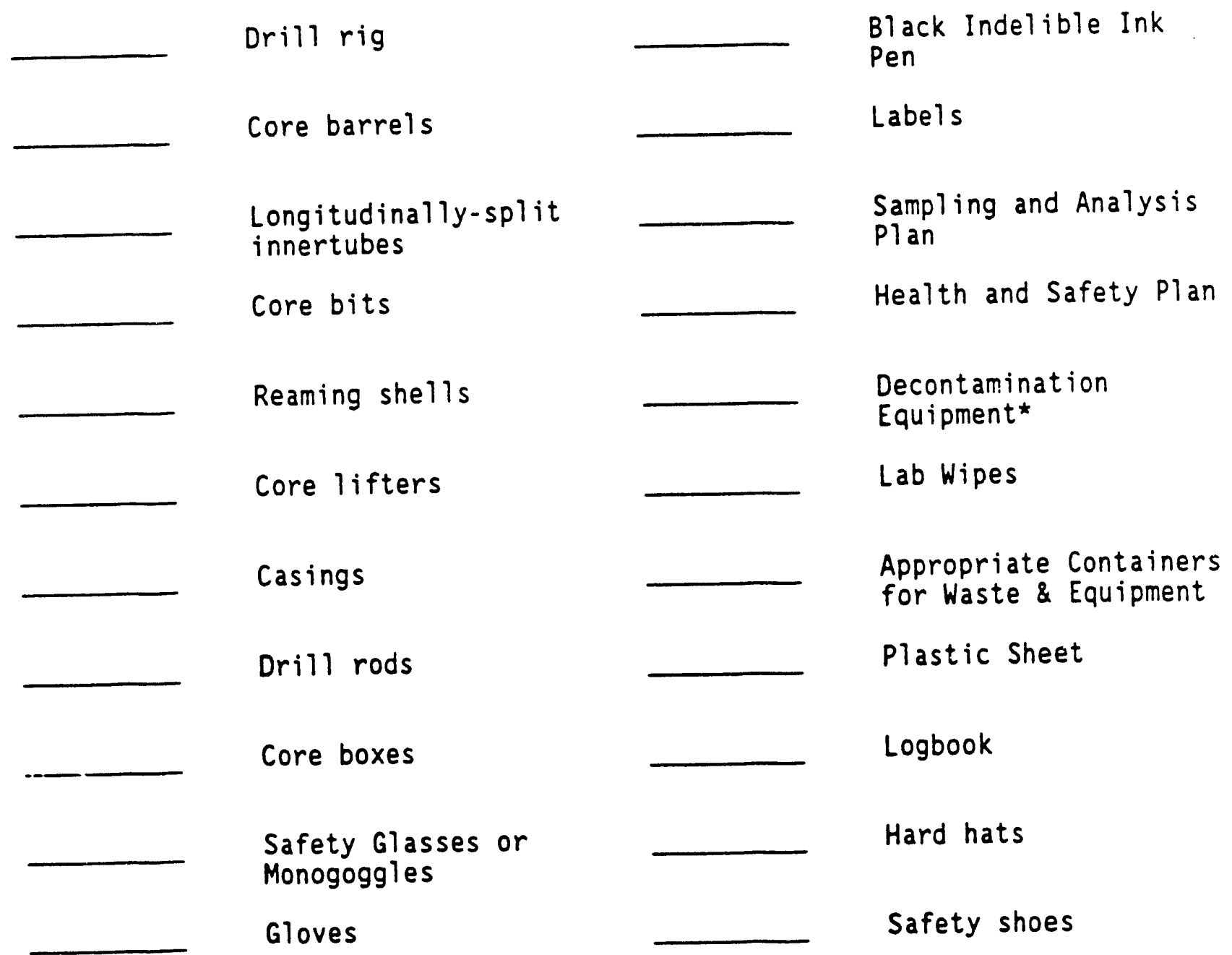


Attachment B, Part B - RCRA Specific Comments Response 34

\author{
Method No.: ESP 303-6 \\ Revision No.: 0 \\ Date: August 31, 1988 \\ Page: 10 of 10
}

MARTIN MARIETTA ENERGY SYSTEMS, INC.

ENVIRONMENTAL SURVEILLAYICE PROCEDURES

Ice/Cooler, as required

Custody seals, as

required

Chain-of-Custody forms

* See Section ESP-901 for decontamination procedures and equipment requirements. 
Attachment B, Part B - RCRA Specific Comments Response 36

ORNL RI/FS PROJECT JOB 19118

\section{PROJECT PROCEDURE REVISION ORDER}

1. PROCEDURE TO BE CHANGED:

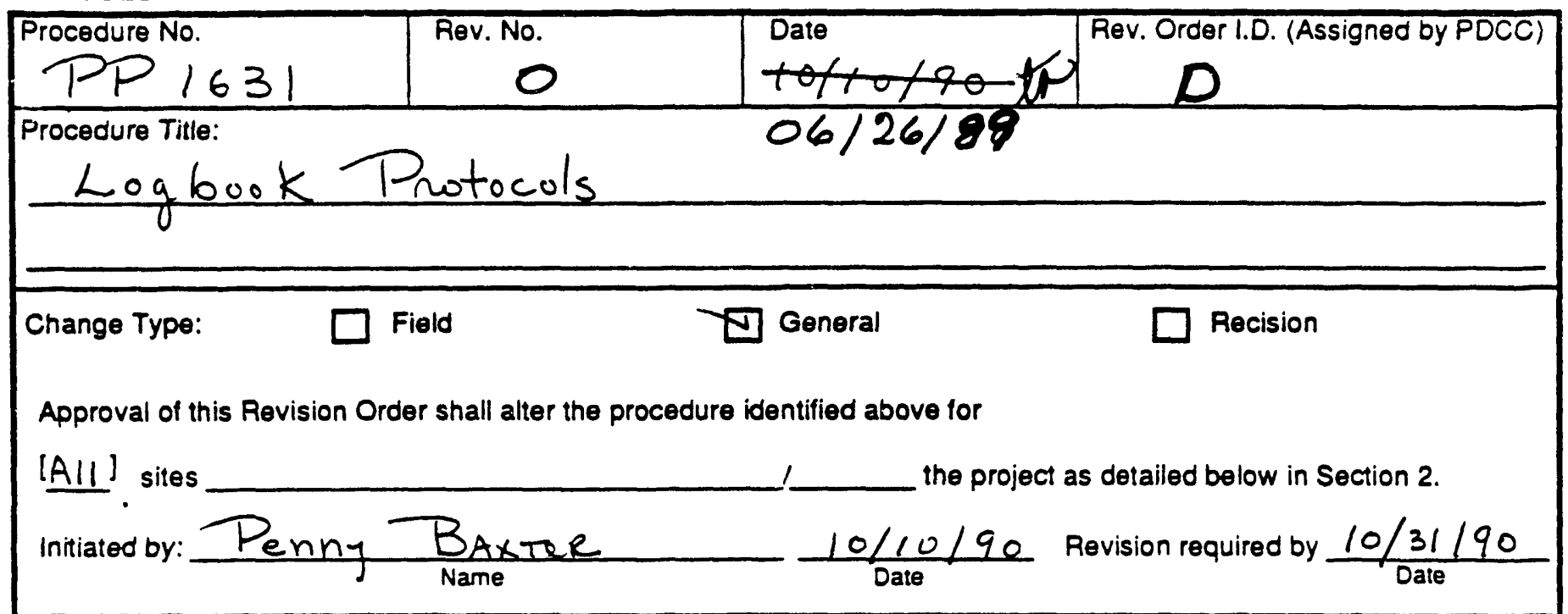

\section{DESCRIPTION OF CHANGE:}

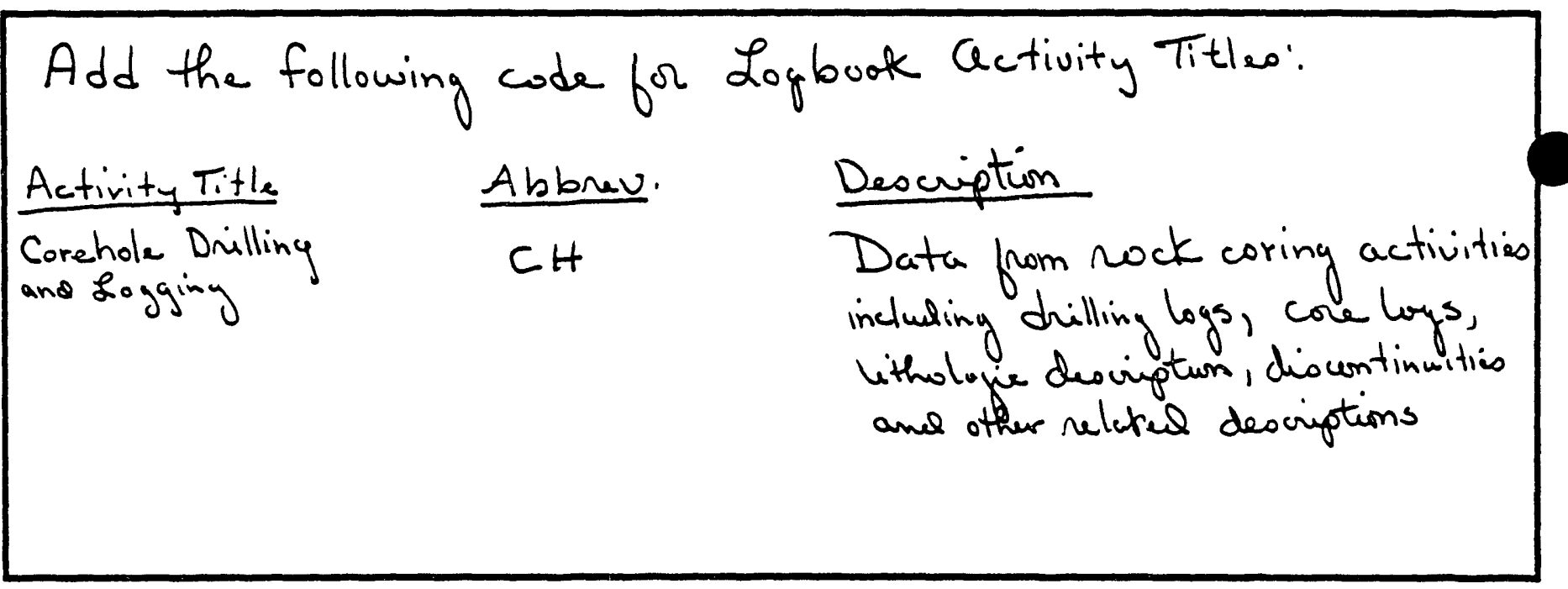

3.

$\bigotimes$ APPROVED

$\square$ MODIF!ED (as noted)

[] DISAPPROVED

\section{$\frac{10 / 10 / 90}{\text { Date }}$

4.

REVIEWED:

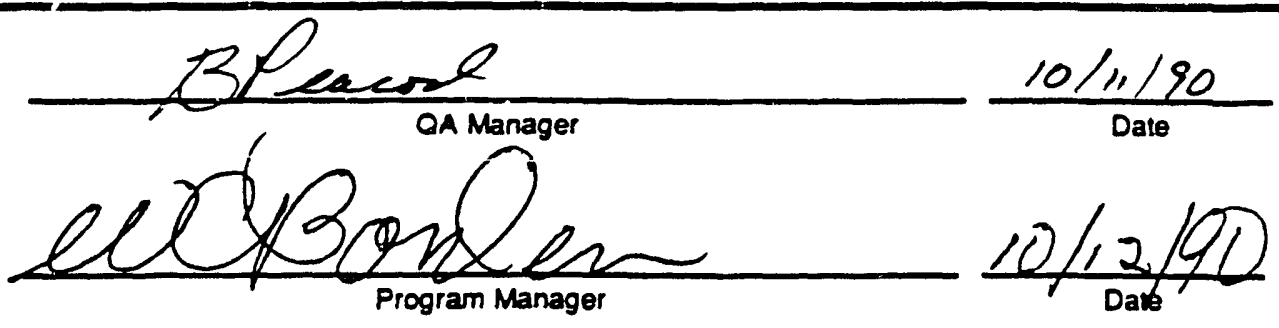

CONCURRED:

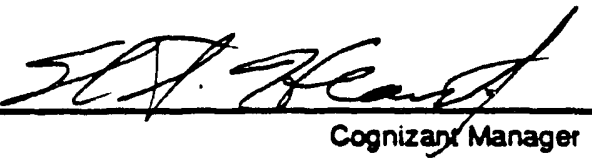


Attachment B, Part B - RCRA Specific Comments Response 36

ORNL RIFS PROJECT JOB 19118

\section{PROJECT PROCEDURE REVISION ORDER}

1. PROCEDURE TO Be ChANGED:

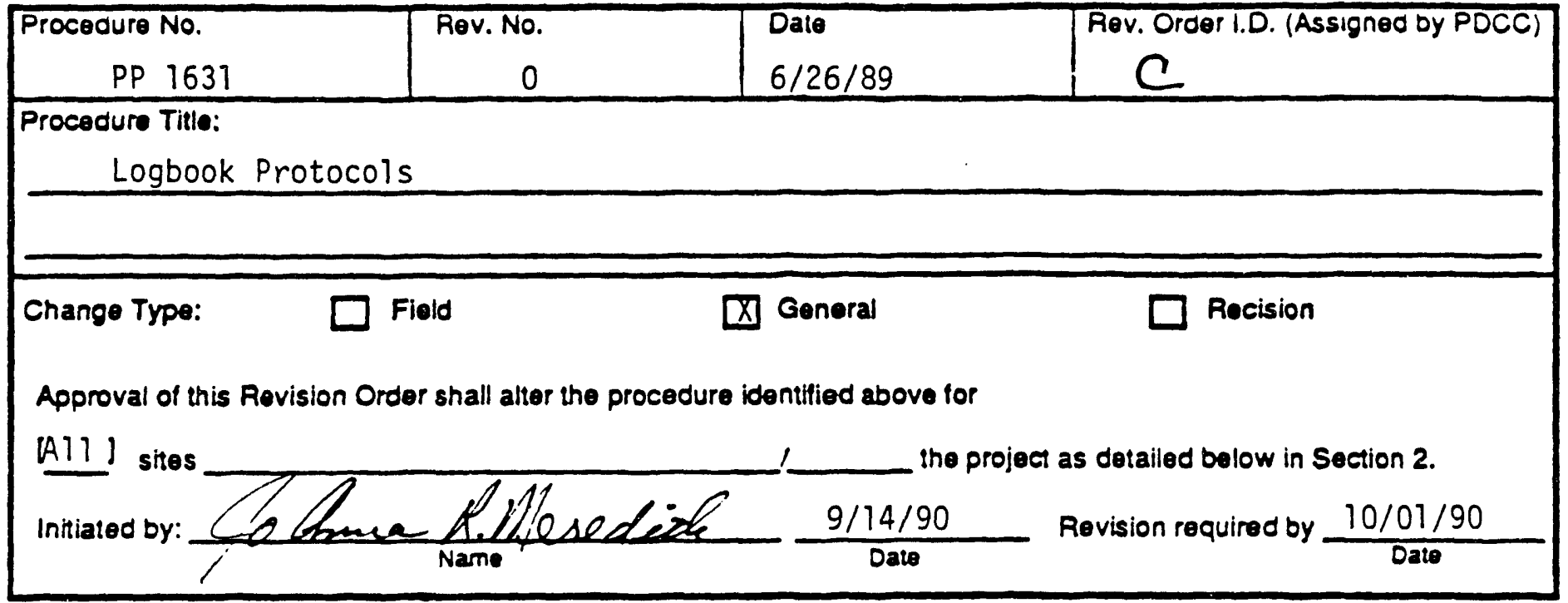

\section{DESCRIPTION OF CHANGE:}

Add the following code for Logbook Activity Titles:

Sample Transfer - ST - For record of sample sets or individual containers transferred to or from the interim storage facility.

Activity Title Abbrev.

Sample Transfer $\quad$-ST-

3.

X APPROVED

MODIFIED (as noted)

DISAPPROVED

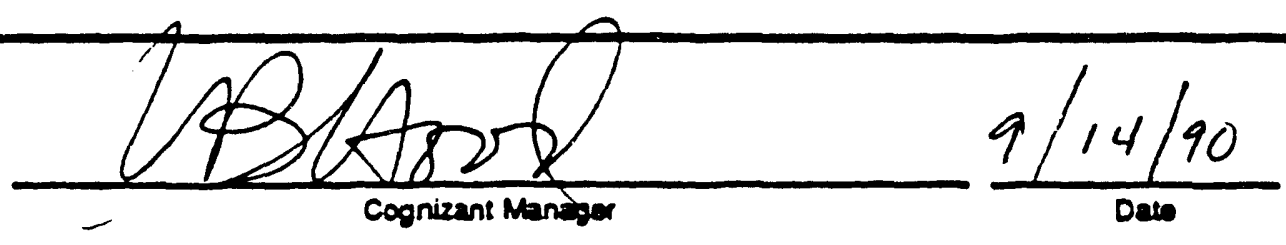

a.

REVIEWED:

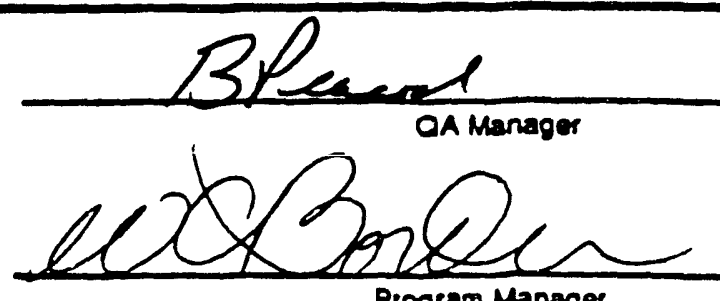

$\frac{9 / 20 / 90}{\text { Dat }}$

Program Managar

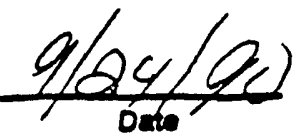


Attachment B, Part B - RCRA Specific Comments Response 36

\section{PROJECT PROCEDURE REVISION ORDER}

1. PROCEDURE tO BE CHANGED:

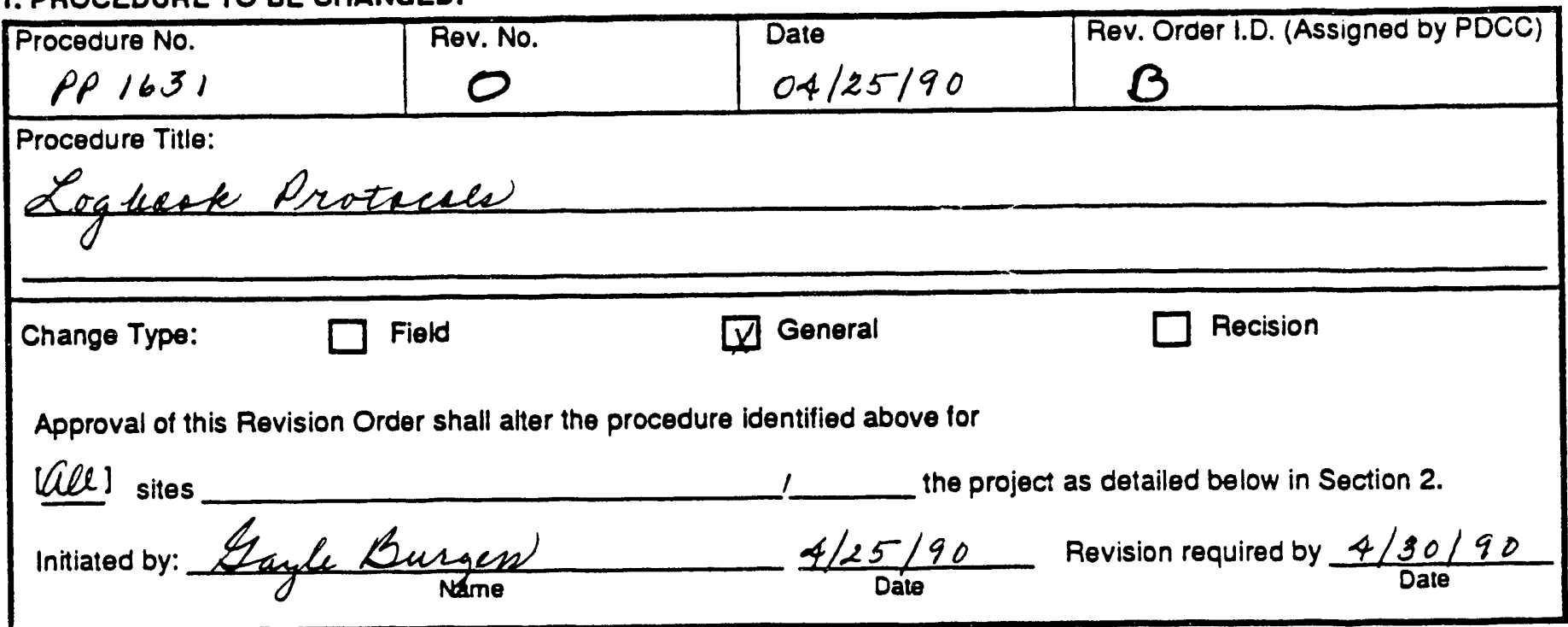

2. DESCRIPTION OF CHANGE:

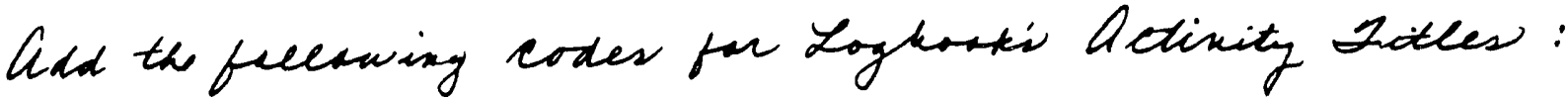

Tank Construetion Activiter - TC - Fier recesd of the desgin and seodifucestion of

undergenend etarege taxks to facieizate sub seguent arsperig.

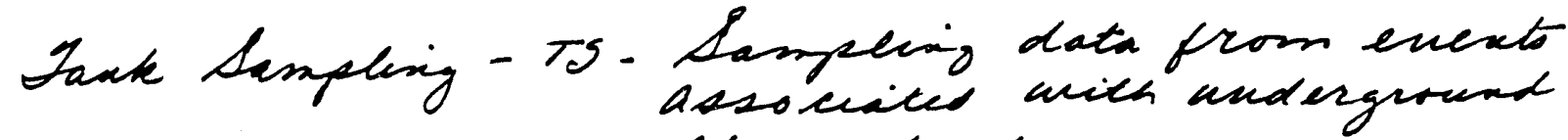
Atarge tanks.

(Continued)

3.

$\nabla$ APPROVED

MODIFIED (as noted)

DISAPPROVED
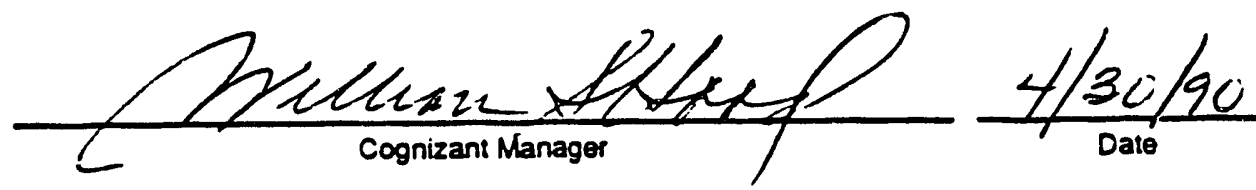

4.

REVIEWED:

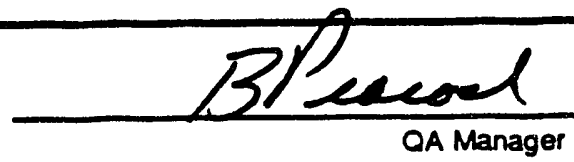

$5 / 2 / 80$

Date

CONCURRED: $M \hat{1}$

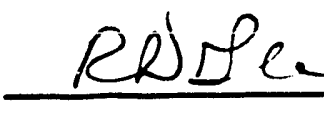

Program Manager

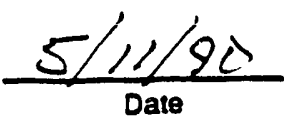


Attachment B, Part B - RCRA Specific Comments

Response 36

ORNL RIFFS PROJECT JOB 19118

\section{PROJECT PROCEDURE REVISION ORDER}

1. PROCEDURE TO BE CHANGED:

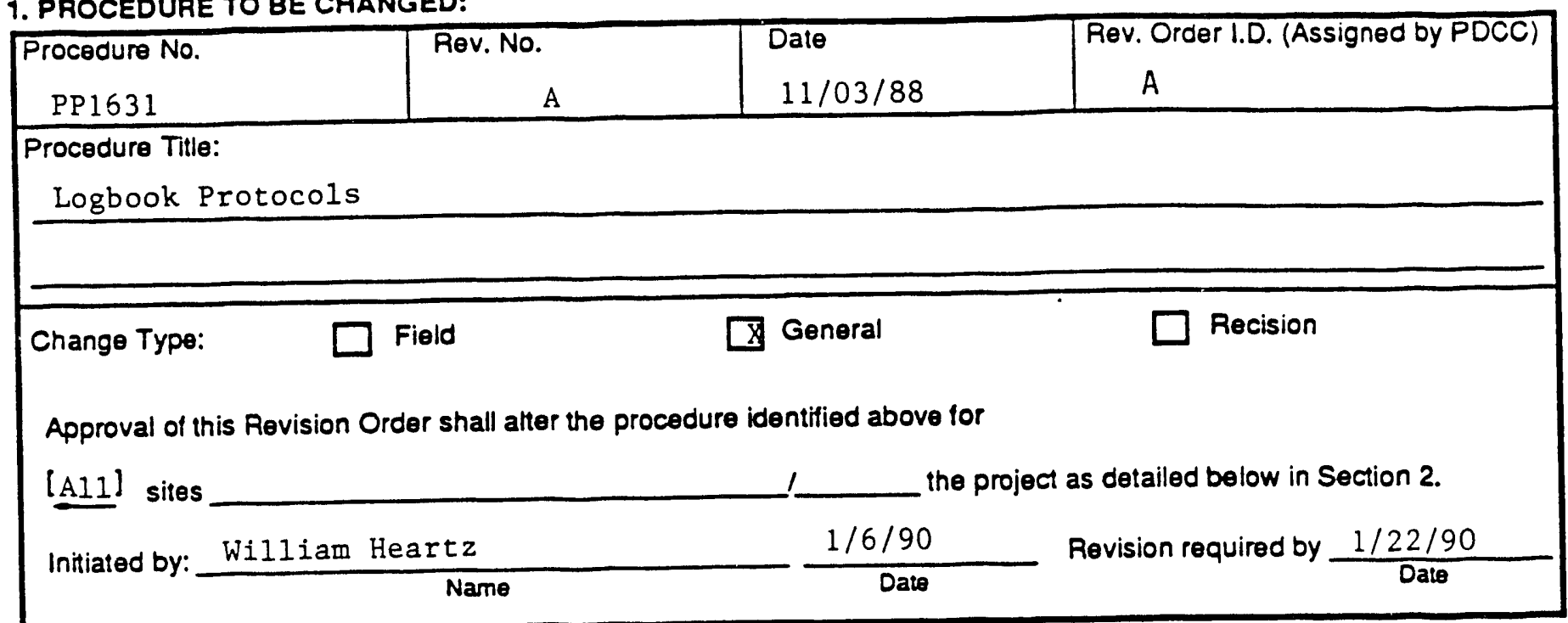

\section{DESCRIPTION OF CHANGE:}

See attached

3.

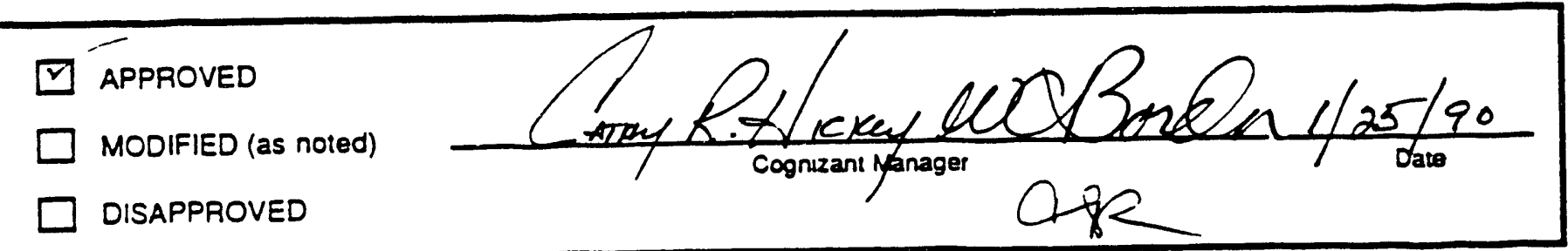

4.

REVIEWED:

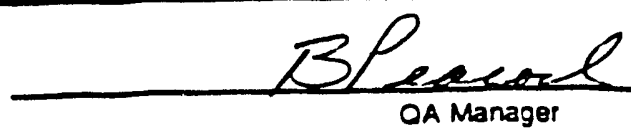

$\frac{1 / 26 / 90}{\text { Date }}$

CONCURRED:

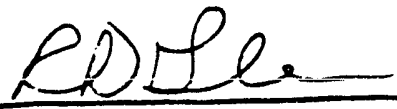

Program Manager

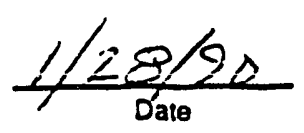


Attachment B, Part B - RCRA Specific Comments Response 36

\subsection{RESPONSIBILITIES}

Change 6.0 on Revision order, Revision A to: "Complete logbooks shall be submitted by the FSS Manager or designee to PDCC within one week of completion. In addition, the FSS Manager or designee shall submit a photocopy of each days logbook to PDCC within one week of the entry. Logbook copies shall be submitted to the FSS Manager or designee at the end of each day." 
Attachment B, Part B - RCRA Specific Comments Response 36

$\begin{aligned} \text { Laboratory Instruments - II - } & \text { Instrument data, calibration data, } \\ & \text { repair and maintenance records of } \\ & \text { Laboratory Equipment. }\end{aligned}$

Sample Receiving

Laboratory

Project Equipment
- SR - Record of sample screening, etc. media for analysis received from the field.

- IR - General laboratory activities

- PE - Instrument data, repair and maintenance of Health Physics equipment including general 
Page 2 of 6

\subsection{RESPONSIBIIITIES}

Add the following sentences to the third paragraph: "Completed logbooks shall be submitted by the FSS Manager or designee to PDCC within 1 week of completion. In addition, the FSS Manager or designee shall submit a xeroxed copy of each day's logbook entries to PDCC within 2 days of the entry."

Page 3 of 6

\subsection{DEFINITIONS}

\section{Iogbook.}

Replace the wording after "Iogbook" and before "Information Form" with the following: The person to whom a new logbook is first issued shall insure that the cover has been clearly marked (using indelible black magic marker), as shown on Attachment 1, with the following information:

- The words "ORNI RI/FS PROJECT LOGBOOK".

- The appropriate WAG Number. Logbooks which do not apply to a single specific WAG should be assigned WAG number 99.

- Logbook Activity Title. A short title which describes the contents of the logbook shall be used. The activity titles are designed to facilitate retrieval of data. All data recorded in a logbook shall be appropriate to the logbook activity title and shall be from the WAG shown on the cover of the logbook. The following is a list of potential logbook activity titles. Not all titles will be needed for all WAGs and additional titles may be added to this list. This procedure shall be revised in accordance with PPI104.1 when other titles are created in order to maintain standardization.

Activity Title

soil Borings

Abbrev.

SB

Well Installation and Development

WI

\section{Description}

Borehole, hand auger and surface soil sampling, geologic description and insitu testing data such as cone penetrometer and geophysical logging.

Data from well, piezometer and wellpoint installation including data from soil borings made as part of a well installation. 
Attachment B, Part B - RCRA Specific Comments Response 36

Groundwater

GW

sampling

Surface Water \&

SW

sediment Sampling

Soil Gas

Well Evaluation

Hydrologic Data

Geophysical

Investigations

Decontamination

Plugging and

Abandonment

Air Sampling

Biota Sampling

Environmental

Health

WE

HD
Data from groundwater sampling events.

Data from surface water and sediment sampling events.

Data from soil gas sampling activities.

Data from evalation of existing wells, such as well head description and head space gas monitoring and sampling.

Data from aquifer testing (pump, slug and packer testing), head measurements (when not part of sampling), surface water hydrology (weir studies), etc.

GI

Data from geophysical investigations such as seismic, electromagnetic and ground penetrating radar.

DC

logs of drilling and sampling equipment decontaminated at the decontamination facility including records of equipment blanks. Field decontamination notes and records of equipment blanks at drilling locations will be included in the sample Team Leader's logbook.

PA

Records of grouting of soil borings and abandonment of wells.

Data from air sampling events.

Sampling of biological specimens.

EH
All ES\&H field logbooks are
included in this category
for purposes of assigning a
consistent numbering system
to all RI/FS field logbooks. 
Attachment B, Part B - RCRA Specific Comments Response 36

Operations

Civil survey

Photographs
OP

CS

$\mathrm{PH}$
Logbook protocols for ES\&H logbooks are found in PP 1265 .

operations logbooks are generally assigned to supervisory and support personnel such as the Fss Manager and Field QC. They will frequently be non WAG specific.

Fieldbooks containing civil survey information pertaining to RI/FS sampling and data collection locations.

Photography logbook protocol is covered in PP 1110.1 . Photograph logbooks are mentioned herein for purposes of assigning consistent numbering to all RI/FS field logbooks.

- Date of first entry in this logbook. The person turning in a completely filled logbook will insure that the date of last entry in the logbook is also shown on the cover.

- Logbook Number. The logbook number shall be of the form:

WAG NO.-

Logbook Activity Title Abbreviation
-Unique Sequential Number

For example, the first logbook for WAG 1 soil borings would be numbered, 01-SB-001. The logbook number should also be lettered on the spine of the logbook if possible to facilitate retrieval from a bookcase.

Page 4 of 6 through 6 of 6 .

\subsection{PROCEDURE}

Add the following sentence to the second paragraph: "Each logbook shall contain a table of contents and a chronological record showing to whom the logbook was issued by date."

Change the word "collection" in the fift.h line of the third paragraph to "correction" and add the words "if necessary".

Add the following words to the second to the last sentence in the 
Attachment B, Part B - RCRA Specific Comments Response 36

third paragraph: "and 1 copy shall be submitted to the FSS Manager. A xeroxed copy of each day's logbook entries shall be submitted by the FSS Manager or designee to PDCC within 2 days of the entry."

Change the sixth paragraph to read: "For each activity the logbook entries will contain, if appropriate, the following:"

Add the following to the end of the paragraph entitled "Task Team Members and Equipment": "The equipment list and calibration information for ES\&H equipment may be entered solely on the ES\&H logbook."

Delete the paragraph entitled "Sample Request Documentation."

In the paragraph entitled "Recorded By (signature)", change the second sentence to read "The signature of the Field Team Leader will be recorded for each work shift's activities." 
Attachment B, Part B - RCRA Specific Comments

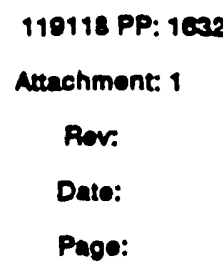

EXAMPLE LOGBOOK COVER

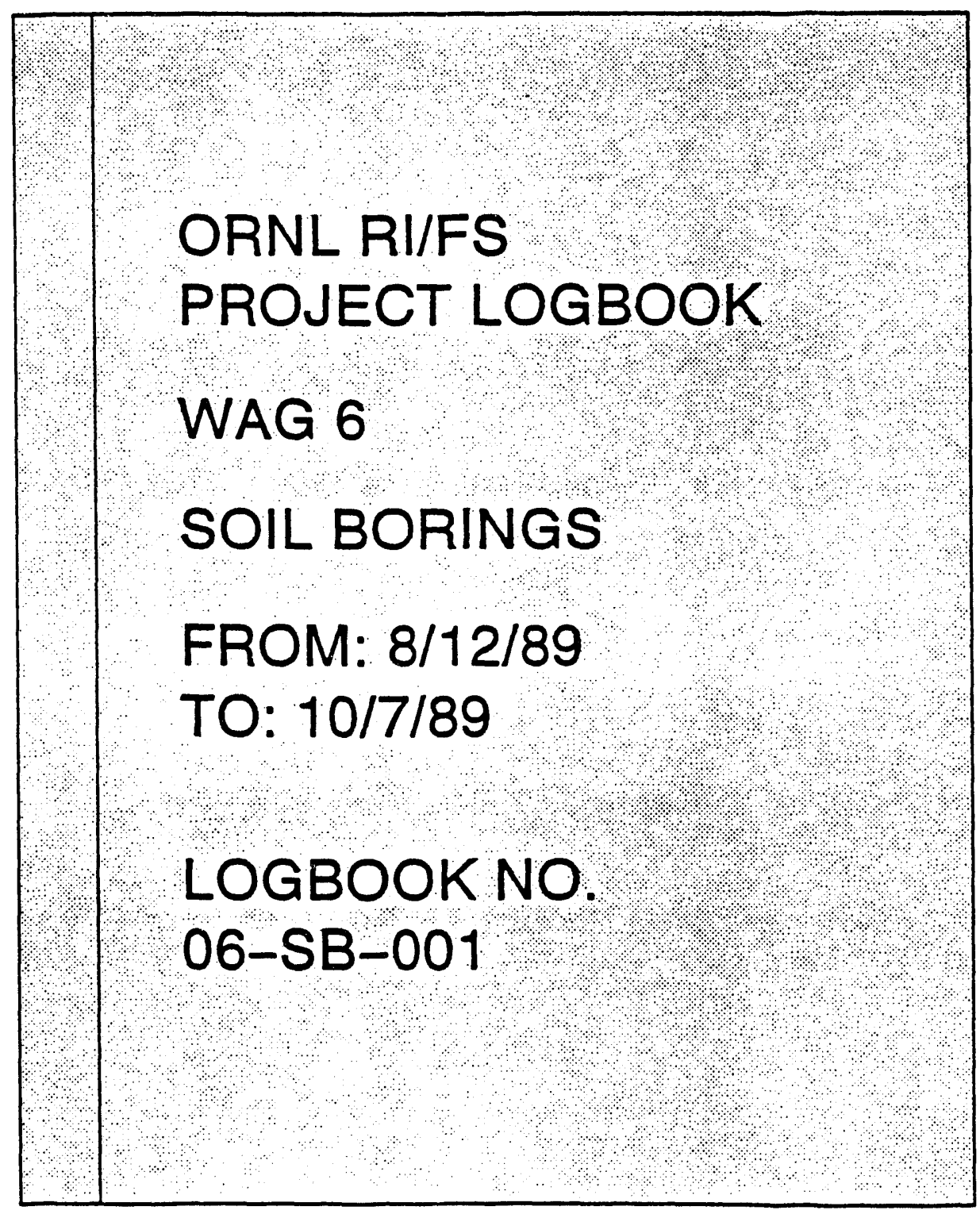


Attachment B, Part B - RCRA Specifi : Comments

Response 36

TITLE:

\section{LOGBOOK PROTOCOLS}

DOCUMENT NUMBER: 1631

REVISION NUMBER: 1

DATE:

03/05/91

PROJECT: ORNL RI/FS

JOB NO.: 19118
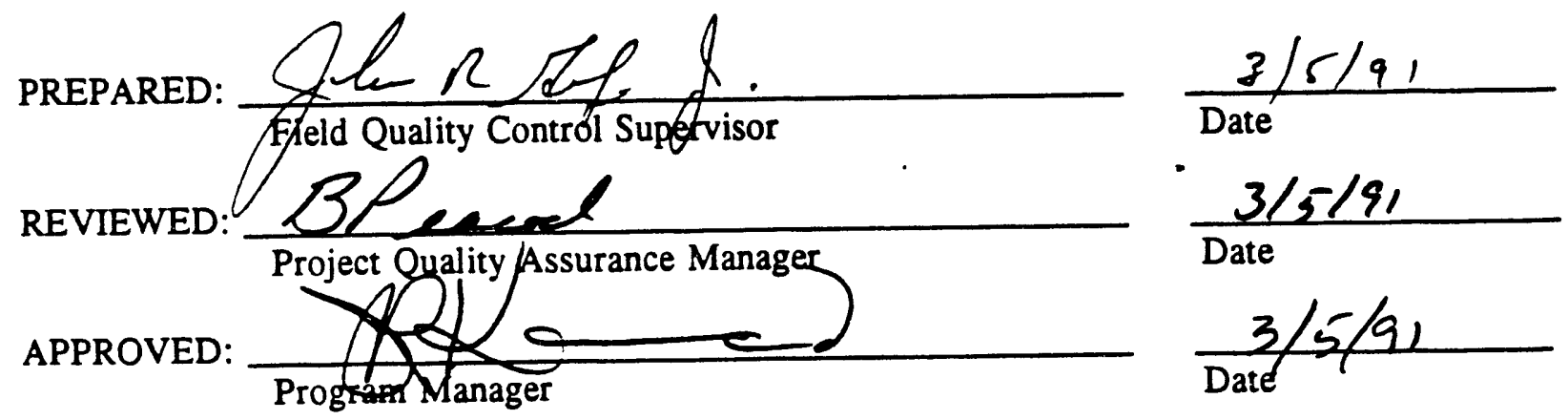
19118 PP: 1631

Rev: 1

Page: 1 of 7

\section{LOGBOOK PROTOCOLS}

\subsection{SCOPE AND APPLICATION}

This method describes the procedures for the identification, use, and control of logbooks and associated field data records for ORNL RI/FS activities. This protocol is applicable to all activities for which the use of field logbooks is specified.

\subsection{REFERENCES}

(1) U.S. Environmental Protection Agency, "A Compendium of Superfund Field Operations Methods, " EPA/540/P-87/001, December 1987.

(2) U.S. Department of Energy, "Environmental Survey Manual," Volume 4, August 1987.

(3) U.S. Environmental Protection Agency, Region IV, "Standard Operating Procedure Quality Assurance Manual," April 1986.

(4) Martin Marietta Energy Systems, Inc., "Environmental Surveillance Procedures Quality Control Program," ESH/Sub/87-!'1706/1 Draft, September 1988.

\subsection{APPLICABLE STANDARDS, REGULATIONS, AND GUIDELINES}

See above.

\subsection{SUMMARY OF METHOD}

Logbooks are used for recording pertinent ORNL RI/FS information, such as the following, or as otherwise described in applicable procedures:

- Documentation of field activities, including equipment used

- Field instrumentation readings

- Health and safety activities

- Laboratory activities

- Photographic references

- Sample chain-of-custody 
- Field calculations

- Significant events (e.g., telephone conversations, meetings, deliverables, correspondence)

\subsection{REOUIRED EOUIPMENT AND APPARATUS}

- Logbook with appropriate data forms. The logbook must be bound and have serialized pages. Data forms may be printed in the logbooks. The logbook will have water repellent pages.

- Indelible black ink pens.

\subsection{RESPONSIBILITIES}

The Program Manager is responsible for ensuring overall compliance with this procedure.

The applicable manager is responsible for determining, in accordance with applicable procedures, which team members will record information in field logbooks and for requesting the necessary logbooks.

The FSS Manager is responsible for the following:

- Obtaining necessary logbooks as requested by the applicable manager

- Maintaining a custody log to document custody of each logbook

- Safeguarding logbooks during the time they are not issued to a user

- Submitting a photocopy of each day's logbook entries to PDCC within one week of the entry

- Distributing additional photocopies of specific logbook entries as required

- Submitting completed logbooks to PDCC within one week of completion. If completed logbooks must be retained for reference (e.g., field survey logbooks), a memorandum will be submitted to PDCC that identifies the completed logbook and the interim custodian.

- Maintaining documentation (e.g., logs) pertaining to logbook issue, transmittal, etc.

The logbook user (field team leader, lead geologist or hydrogeologist, field sampler, field safety technician, laboratory technician designated by the applicable manager) is responsible for the following:

- Signing the logbook out from the custodian and returning it daily after use

PP1631.R1 
19118 PP: 1631

Rev: 1

Page: 3 of 7

- Recording pertinent data legibly, as it is obtained, in the logbook in accordance with applicable procedures and this protocol

- Protecting the logbook from physical damage or chemical or radiological contamination

- Maintaining strict possession of the logbook to preclude loss or tampering

- Providing to the logbook custodian at the end of each day, a photocopy of each page upon which data was entered or revised.

Applicable supervision (e.g., Assistant WAG Manager, FHS Manager, FSS Manager) is responsible for periodically reviewing data entries in each logbook used to record activities under their supervision or direction, as applicable, for accuracy, reasonableness, and consistency. These reviews should be performed weekly, or more often depending on activity levels, and shall be documented as follows:

- The designated reviewer completes the review form (Attachment 1) and submits it to the Field Clerk or other designated control point.

- The Field Clerk distributes the original review form to the logbook author, one copy to the Field QC Supervisor, and retains a copy of the form in a suspense file.

- The logbook author coordinates comments, as required, with reviewer, makes any necessary corrections in logbook, signs the original of the review form, and forwards the review form to the Field QC Supervisor.

- The Field QC Supervisor verifies that the necessary corrections have been incorporated into logbooks, and signs the review form. The Field QC Supervisor coordinates, as required, with original reviewer and the logbook author to resolve any concerns.

- The Field QC Supervisor returns the completed review form to Field Clerk for transmittal to PDCC. One copy of the completed form will be retained by the Field Clerk for reference, and one copy will be forwarded to the original reviewer to indicate completion.

The Field QC Supervisor is responsible for inspecting logbook entries to determine compliance with this procedure and to establish that the entries are of a quality to meet the needs and requirements of the RI/FS.

\subsection{REQUIRED PRACTICES}

\subsection{SAFETY}

Not applicable.

PP1631.R1 


\subsection{CONTAMINATION CONTROL}

To the extent possible, the logbook should be protected from contamination (e.g., by carrying it in a plastic bag or in an engineer's metal clipboard that closes over the logbook). Persons with contaminated gloves or protective clothing should not handle the logbook. Should the logbook become contaminated, it is decontaminated (dried, blotted) or placed in clear plastic bags so that entries may still be read. If the logbook is contaminated to such extent, as determined by the ES\&H Manager or designee, that it cannot be removed from the site, it will be hand-copied onto the appropriate data forms at the site. The circumstances warranting this action must be noted on the rewritten form.

\subsection{DEFINITIONS}

Logbook. A field notebook with consecutively numbered pages, which will be bound to prevent removal of pages. The logbook shall be identified as described below.

The cover shall be clearly marked (e.g., using indelible black magic marker), as shown on Attachment 2, with the following information:

- The words "ORNL RI/FS PROJECT LOGBOOK."

- The appropriate WAG number. Logbooks pertaining to underground storage tank activities shall be assigned WAG number 91 . Logbooks that do not apply to a single specific WAG shall be assigned WAG number 99.

- Logbook Activity Title. A short title that describes the content of the logbook shall be used. The activity titles are designed to facilitate retrieval of data. See Attachment 3 for a list of logbook activity titles. Not all titles will be needed for all WAGs. Additional titles may be added to this list by revision in accordance with PP 1114.1.

- Date of first entry in this logbook. The person turning in a completely filled logbook make sure that the date of last entry in the logbook is also shown on the cover.

- Logbook Number. The logbook number shall be of the form

$$
\begin{array}{ll}
\text { WAG No.- } & \begin{array}{l}
\text { Logbook Activity } \\
\text { Title Abbreviation }
\end{array} \\
& \text {-Unique Sequential } \\
\text { Number }
\end{array}
$$

For example, the first logbook for WAG 1 soil borings would be numbered 01-SB-001. The logbook number should also be lettered on the spine of the logbook, if possible, to facilitate retrieval from a bookcase.

Information Form. Produced data entry forms that list the data needs for a particular task or event. Information forms serve two purpooses:

PP1631.R1 
Rev: 1

Page: 5 of 7

- They act as reminders of specific data needs.

- They aid in data entry in the RI/FS data base.

Information forms include well completion diagrams, soils classification charts, and other basic data entry forms.

\subsection{PROCEDURE}

The field logbook serves as the primary record of field activities. Entries are made chronologically in sufficient detail to allow reconstruction of the events without reliance on memory.

The field logbook user is issued the appropriate logbook at the beginning of each day's activities by the assigned custodian. Each logbook shall contain a table of contents and a chronological record showing to whom the logbook was issued by date. In addition, the logbook number shall be entered at the top of each left-hand page to facilitate identification of individual photocopied pages.

All field descriptions and observations are recorded in the logbook using consecutive pages (do not skip pages). All entries recorded in a logbook shall be appropriate to the logbook activity title and shall be applicable to the WAG shown on the cover of the logbook. Observations are noted in indelible black ink; any entry errors are corrected by drawing one solid line through the incorrect entry which is then initialed by the logbook user along with a written explanation of the correction if necessary. At the end of each day, the logbook entries are photocopied by the user. The logbook and photocopy shall be submitted to the logbook custodian daily. The user may retain a copy for information. A photocopy of each day's logbook entries shall be submitted by the FSS Manager to PDCC within 1 week of the entry. Additional copies shall be distributed as required.

The field notes may be transcribed by the users onto clean information forms at the completion of each activity. The clean copy is used for data base entry. Both the logbook and the clean copy are submitted to, and checked for consistency by, appropriate supervision. To prevent the release of unverified data, all requests for information or data prior to data base entry must be routed to the WAG manager.

The logbook user is responsible for completing necessary reports detailing sampling errors or omissions.

For each activity, the logbook entries will contain, if appropriate, the following types of information:

Logbook Number. Enter the logbook number at the top of each left-hand page.

Name of Activity. Subsurface Soil Sampling, Groundwater Monitoring, Well Installation, etc.

Task Team Members and Equipment. Name all the members on the field team involved in the specified field activity(s). List equipment used by serial number or other identifiers; include 
19118 PP: 1631

Rev: 1

Page: 6 of 7

information on calibration. The equipment list and calibration information for ES\&H equipment may be entered solely on the ES\&H logbook.

Activity Location. Indicate location of sampling area as referred to in the Field Sampling Plan and as marked on the field stake placed by the civil survey (if appropriate).

Weather. Indicate general weather and precipitation conditions.

Methods. Indicate method/procedure numbers employed for activity.

Sample Number(s). Indicate the unique number(s) associated with the physical sample(s) along with the sample(s) or bar code label(s) collected, if applicable. Identify QC samples.

Sample Type and Volume(s). Indicate the media, the container type, the preservative, and the volume for each sample.

Sample Depth or Interval. Indicate the depth interval from which each sample is collected (e.g., $1.0 \mathrm{ft}-1.5 \mathrm{ft}$ ).

Time and Date. Record the time and date (time/dd/mm/yy) when the activity was performed (e.g., 0830/27/JUN/87). Time is expressed in the 24-h (military) notation, where the hours of $1 \mathrm{p} . \mathrm{m}$. to 11 p.m. are represented by 1300 to 2300 hours, where noon is 1200 , and midnight is 2400 . In recording the date, the day and year must be recorded using two numeric digits each. For the first nine days of the month, the numbers 01 through 09 should be used. This procedure should be followed whenever a date is recorded.

Analyses. Indicate the appropriate code for analyses to be performed on each sample, as specified in the Sample Event Initiation Request (SEIR).

Field Measurements. Indicate measurements and field instrument readings taken during activity.

Chain of Custody and Distribution. Indicate chain of custody control number for each sample collected and indicate to whom samples are distributed and the user.

References. If appropriate, indicate references such as maps or photographs employed in the activity.

Narrative (including time and location). Create a factual, chronological record of the team's activities throughout the day, including the time and location of each activity. Include descriptions of any general problems encountered and resolutions found. Provide the names and badge numbers of nonfield team personnel who visit the site, request changes in activity, impact the work schedule, request information, or observe team activities. Record any visual or otherwise sensed observations relevant to the activity, the waste source, or the sample itself.

PP1631.R1 
Attachment B, Part B - RCRA Specific Comments Response 36

19118 PP: 1631

Rev: 1

Page: 7 of 7

Data will be carefully recorded in appropriate units and in sufficient detail to allow any other worker to continue the data collection with the same degree of accuracy.

It should be emphasized that logbook entries are for data recording and chronologies of events. As a part of this, the logbook author must include observations and descriptive notations. However, care must be taken to be objective and record no opinions or subjective comments.

Recorded By (signature). Indicate the individual responsible for the entries contained on the form or in the logbooks. The dated signature of the logbook user will be recorded at the bottom of each page.

In addition, there are individual data needs tailored to separate tasks (e.g., soil logging, water sampling, well installation, etc.). These data needs are listed on data base information forms. All items listed on these forms must be addressed.

\subsection{ATTACHMENTS}

Attachment 1: Review form

Attachment 2: Logbook cover

Attachment 3: Logbook activity titles 
Attachment B, Part B - RCRA Specific Comments Response 36

19118 PP: 1631

Attachment: 1

Rev: 1

Date: $03 / 05 / 91$

Page: 1 of 1

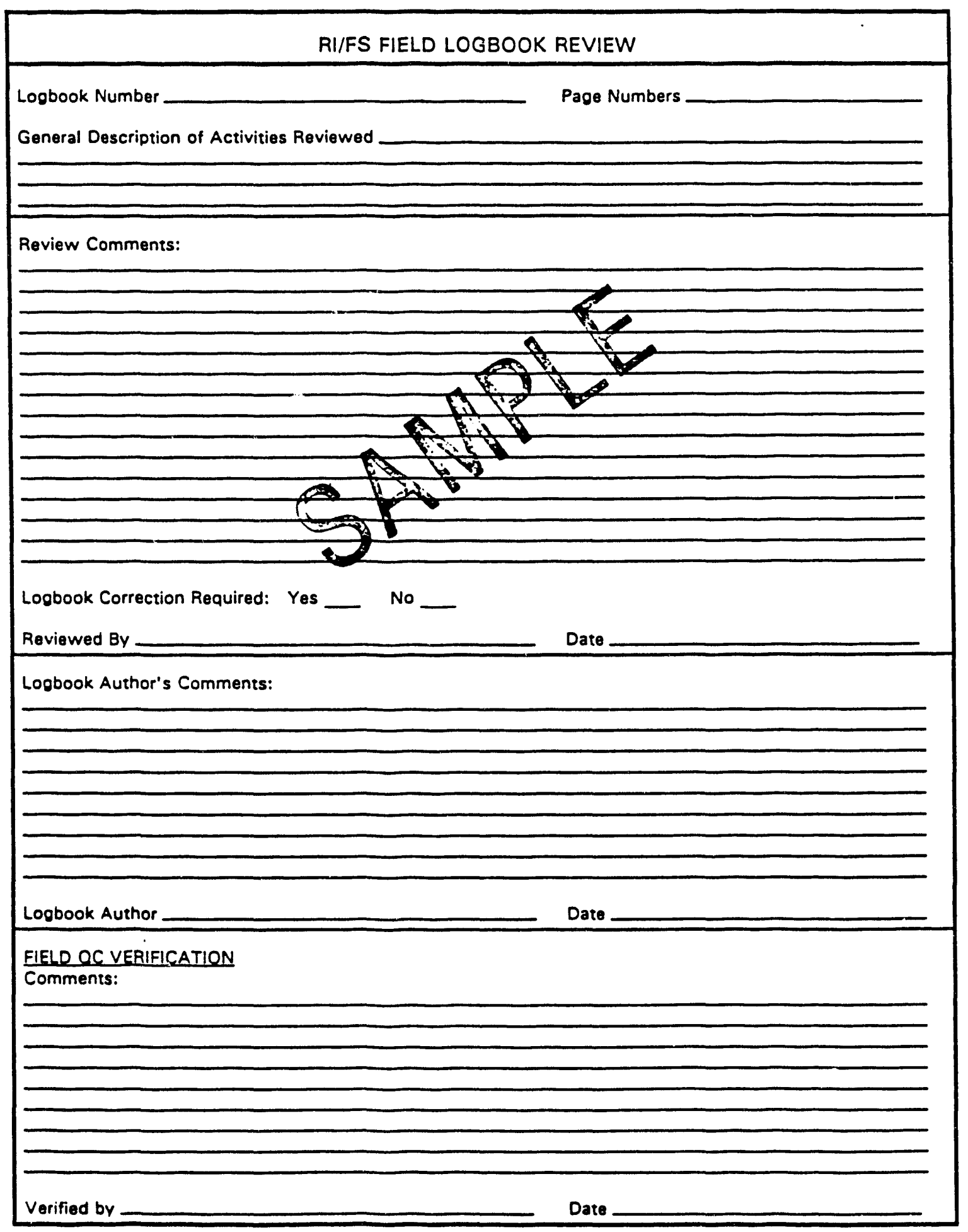

PFió3i.Ri 
Attachment B, Part B - RCRA Specific Comments

Response 36

19118 PP: 1631

Attachment: 2

Rev: 1

Date: $03 / 05 / 91$

Page: 1 of 1

EXAMPLE LOGBOOK COVER

\section{ORNL RI/FS}

PROJECT LOGBOOK

WAG 6

SOIL BORINGS

FROM: $8 / 12 / 89$

TO: $10 / 7 / 89$

LOGBOOKNO.

06-SB-001 
19118 PP: 1631

Attachment: 3

Rev.: 1

Date: $03 / 05 / 91$

Page: 1 of 3

\section{LOGBOOK ACTIVITY TITLES}

Activity Title

Air Sampling

Biota Sampling

Corehole Drilling and

Logging

Civil Survey

Database Operations

Decontamination

Environmental Health

Geophysical Investigations

Groundwater Sampling

Hydrologic Data

Laboratory Instruments

Laboratory
Abbreviation

AS

BS

$\mathrm{CH}$

CS

DB

DC

EH

GI

GW

HD

LI

LR

\section{Description}

Data from air sampling events.

Sampling of biological specimens.

Data from rock coring activities including drilling logs, core logs, lithologic description, discontinuities and other related descriptions.

Civil survey information pertaining to R/FS sampling and data collection locations.

Record of actions taken to define, construct, or alter database structure or content.

Logs of drilling and sampling equipment decontaminated at the decontamination facility including records of equipment blanks. Field decontamination notes and records of equipment blanks at drilling locations will be included in the Sample Team Leader's logbook.

All ES\&H field logbooks are included in this category for purposes of assigning a consistent numbering system to all RI/FS field logbooks. Logbook protocols for ES\&H logbooks are found in PP 1265.

Data from geophysical investigations such as seismic, electromagnetic, ground penetrating radar and USRADS.

Data from groundwater sampling events.

Data from aquifer testing (pump, slug and packer testing), head measurements (when not part of sampling), surface water hydrology (weir studies), etc.

Instrument data, calibration data, repair, and maintenance records of laboratory equipment.

General laboratory activities.

PP1631.R1 
19118 PP: 1631

Attachment: 3

Rev.: 1

Date: 03/05/91

Page: 2 of 3

Operations

Plugging and Abandonment

Project Equipment

Photographs

Soil Borings

Soil Gas

Sampling Receiving

Sample Transfer

Surface Water and Sediment Sampling

Tank Construction Activities

Tank Sampling

Well Evaluation
OP

PA

PE

PH

SB

SG

SR

ST

SW

TC

TS

WE

Operations logbooks are generally assigned to supervisory and support personnel such as the FSS Manager and Field QC Supervisor. They will frequently be non-WAG specific.

Records of grouting of soil borings and abandonment of wells.

Instrument data, repair, and maintenance of measuring and test equipment including general activities.

Photography logbook protocol is covered in PP 1110.1. Photograph logbooks are mentioned herein for purposes of assigning consistent numbering to all RI/FS field logbooks.

Borehole, hand auger, and surface soil sampling, geologic description, and in situ testing data such as cone pentrometer and geophysical logging.

Data from soil gas sampling activities.

Record of sample screening, etc., media for analysis received from the field.

Record of sample sets or individual containers transferred to or from the Interim Storage Facility.

Data from surface water and sediment sampling events.

Field record of the design and modification of underground storage tanks to facilitate subsequent sampling.

Sampling data from events associated with underground storage tanks.

Data from evaluation of existing wells, such as well head description and head space gas monitoring and sampling. 
Attachment B, Part B - RCRA Specific Comments Response 36

\author{
19118 PP: 1631 \\ Attachment: 3 \\ Rev.: 1 \\ Date: $03 / 05 / 91$ \\ Page: 3 of 3
}

Well Installation and Development
WI

Data from well, piezometer and wellpoint installation including data from soil borings made as part of a well installation. 


\section{PROJECT PROCEDURE REVISION ORDER}

1. PROCEDURE TO BE CHANGED:

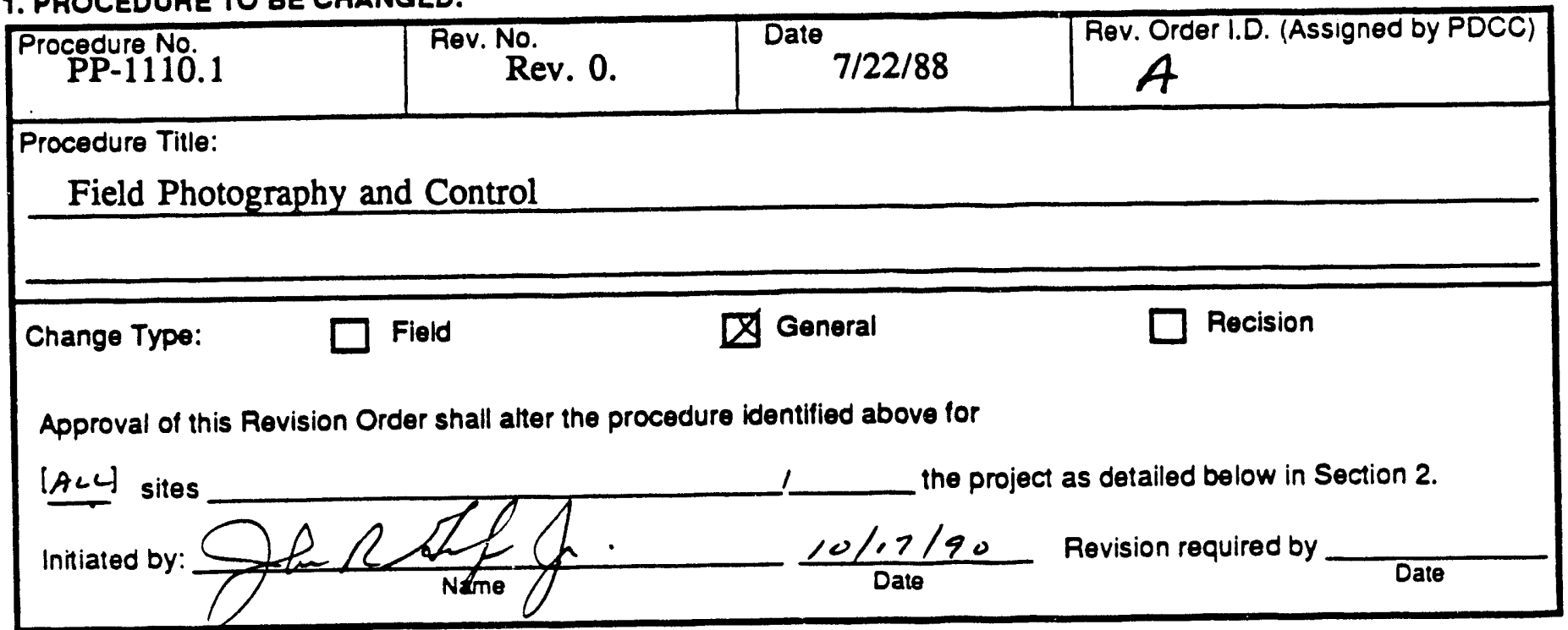

2. DESCRIPTION OF CHANGE:

Page 1, Section 4.0. Add the following immediately following the last sentence of the paragraph:

"Field logbooks shall be utilized and maintained in accordance with PP-1631, "Logbook Protocols," and the following:

- A separate photograph logbook shall be maintained for each WAG.

- When possible, specific cameras will be assigned to specific logbooks (WAGs).

- In no case shall photographs applicable to more than one WAG appear on the same roll of film. If a camera needs to be cleared, photograph a signboard signifying the frame number representing the end of the roll, then rewind the film for processing and insert a new roll for the next use. (This does not apply to instant pictures.)"

3.

$\nabla$ APPROVED

MODIFIED (as noted)

DISAPPROVED

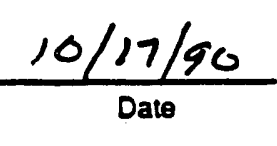

4.

REVIEWED:

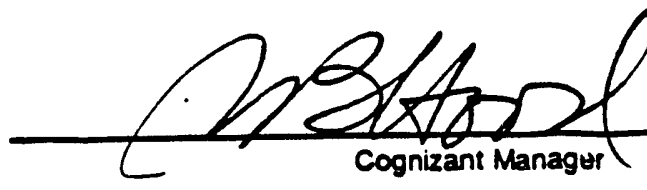

$\frac{10 / 17 / 90}{\text { Date }}$

L

REVIEWED:

CONCURRED:

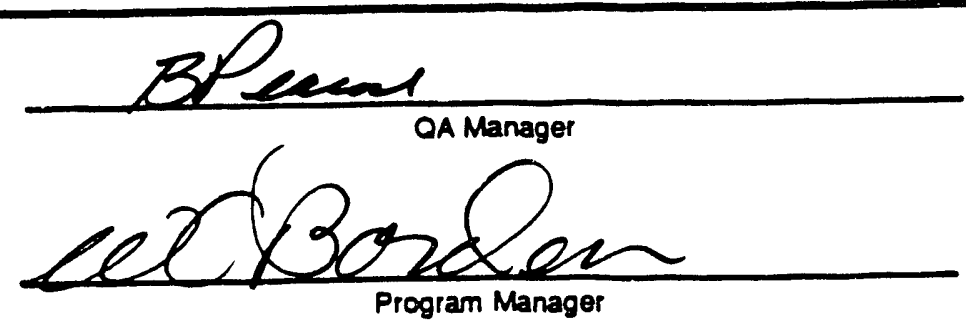

$\frac{10 / 18 / 90}{\text { Date }}$

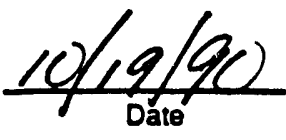


Attachment B, Part B - RCRA Specific Comments Response 37

TITLE:

DOCUMENT NUMBER:

REVISION NUMBER:

DATE :
FIELD PHOTOGRAPHY AND CONTROL

1110.1

0

$07 / 22 / 88$

PROJECT: ORNL RI/FS

JOB NO.: 19118

PREPARED:

Barhara A. Hood Project Administrative Supervisor

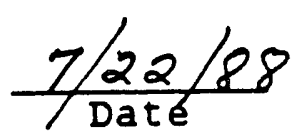

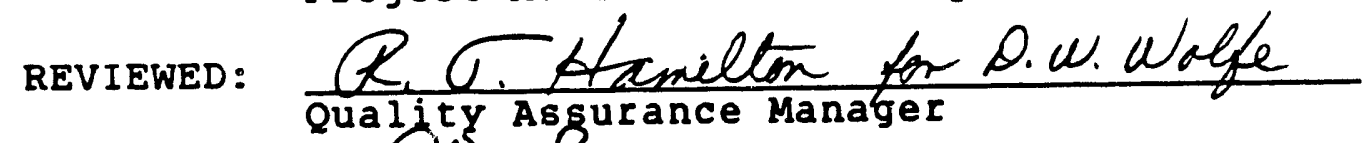
$\frac{7 / 22 / 88}{\text { Date }}$

APPROVED:

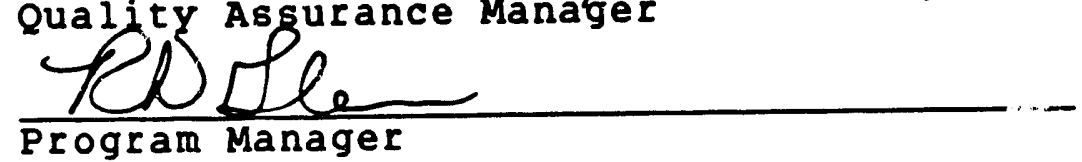

$\frac{7 / 22 / 85}{\text { Date }}$ 
Attachment B, Part B - RCRA Specific Comments Response 37

$$
\begin{aligned}
.9118 \text { PP: } & 1110.1 \\
\text { Rev: } & 0 \\
\text { Date: } & 07 / 22 / 88 \\
\text { Page: } & 1 \text { of } 5
\end{aligned}
$$

\section{FIELD PHOTOGRAPHY AND CONTROL}

\subsection{PURPOSE}

This procedure describes the program used to provide photographic documentation of ORNL RI/FS field activities.

\subsection{SCOPE}

The photographic documentation program includes control of unexposed film, processing, photographs, and negatives on the ORNL RI/FS project.

\subsection{DEFINITIONS}

Film Custodian - The FSS Manager, or designee.

\subsection{PROCEDURE}

In many instances, field investigators must take photographs of field activities including field sampling and location, excavation work. etc. Investigators will record information about the photographs regarding date, time, project, orientation, and name of person taking the photograph. This information is recorded in the field log book, which is included in the field records.

4.1 A standard $35 \mathrm{~mm}$ camera capable of providing clear, sharp photographs is preferred. A low-cost, rugged camera is preferred due to rough usage and the probability of loss due to contamination.

Typical equipment used includes:

- $35 \mathrm{~mm}$. single lens reflect camera, with $50 \mathrm{~mm}$ non-thoriated lens and UV filter: equipped with data back

- "Instant picturen camera, Polaroid 600 or equivalent

- Video, vHs format, $1 / 2$ or $3 / 4$ inch

- "Point and shoot" (disposable) 110 carcridge or $35 \mathrm{~mm}$ camera The preferred film is Kodachrome (Kodak) print ASA 200 file, 24-exposure rolls, or equivalent. 
Attachment B, Part B - RCRA Specific Comments Response 37

$$
\begin{aligned}
19118 \text { PP: } & 1110.1 \\
\text { Rev: } & 0 \\
\text { Date: } & 07 / 22 / 88 \\
\text { Page: } & 2 \text { of } 5
\end{aligned}
$$

4.2 Film is stored under the direction of and issued by the film custodian in a cool. dry place prior to being exposed and after exposure until processing. Cameras and photographic equipment is controlled and issued by the film custodian.

4.3 The field film custodian assigns a unique roll number by WAG for each roll of unexposed film issued to field personnel. A list including roll number, name of photographer, date issued, and date returned is maintained by the film custodian.

4.4 After exposure, the film is returned to the film custodian for processing. Processing is provided through a local photo shop that provides high quality printing and developing. Two $3.5 \mathrm{x}$ 5-in. color prints are procured. Film will be developed in a manner to demonstrate an acceptable degree of control; chain of custody procedureo ale used only if required.

4.5 Each photograph is assigned a unique number as shown below:

$\begin{array}{ccc}\mathbf{x x} & \mathbf{x x x} & \mathbf{x x} / \mathbf{x x} / \mathbf{x x} \\ \text { WAG No. } & \text { Roll No. Nog. No. May/Year }\end{array}$

For example: $05-003-15-5 / 16 / 88$ is the ID number for a photograph from WAG 5, third roll, negative number 15 , taken on May 16, 1988.

Note: Use the negative number, not the sequence number, in the roll. The corrective negative number is required to select the proper negative when additional prints are required.

Roll numbers are consecutive for each WAG (WAG number and consecutive roll number are both required for proper identification).

Both sets of photographs are numbered in accordance with the above on the back of each photograph along with a description of the photograph containing:

- Site name, unique photograph identification number, time, date, and weather conditions.

- Complete description of activity shown: identify the subject and añy individuals shown. Explain why the photograph was caken. (Locations may be identified as numbers keyed to a location on a site map or sketch. The orientation of the photograph should be included.)

- Photographer's signature. 
Attachment B, Part B - RCRA Specific Comments Response 37

$$
\begin{aligned}
19118 \text { PP: } & 1110.1 \\
\text { Rev: } & 0 \\
\text { Date: } & 07 / 22 / 88 \\
\text { Page: } & 3 \text { of } 5
\end{aligned}
$$

Both sets of photographs are placed in plastic photograph holders. One set is retained by the WAG manager. The second set along with negatives are sent to the project Administrative supervisor for filing. The Project Administrative Supervisor will file this copy of the photographs in binders sequentially bY WAG.

4.6 Only personnel named on valid camera permits will be allowed to carry a camera during any ORNL field activities. If the photographs are to be used to document sampling prorsdures or to serve as evidence, the following procedure will ke used:

- The first and last frames of the roll of film will be of a signboard containing the WAG number, roll number, photographer's name, and beginning or ending date and time.

- No special lenses or filters, other than a UV filter, shall be used.

- Any serial numbers for the lens and camera body or property control numbers for the camera will be entered in the logbook.

- Photographs will be composed to show a scale object and, if possible, orientation on the site (i.e.., a prominent landmark). If samples (i.e.. soil cores) are photographed, a sign board showing WAG number, date/time of collection, sample number, and depth or other information concerning location will be included in the photograph.

- Photographs of sampling activities should be composed to show the techniques used, any particular QC methods employed, and individuals conducting the sampling.

- Use a data back equipped camera whenever possible. The data back automatically records date and time on each photograph as it is taken. "Instant picture" and video cameras are used to record activities on site, either to assist in preparing reports or to be used to show non-field-rated project participants what field conditions are like.

4.7 Field log book entries will contain the following information:

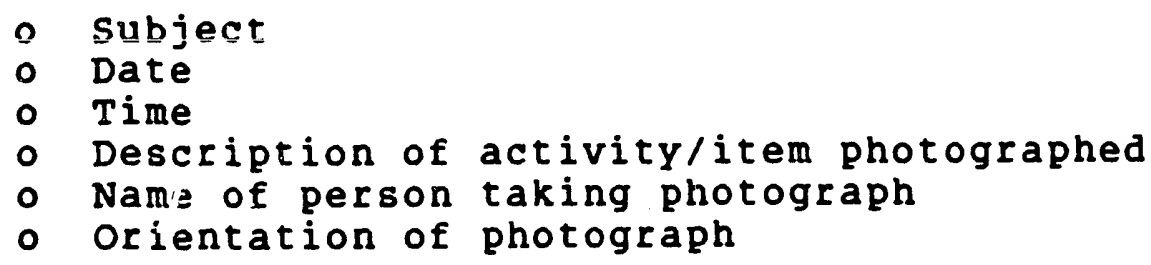


Attachment B, Part B - RCRA Specific Comments Response 37

$$
\begin{aligned}
19118 \text { PP: } & 1110.1 \\
\text { Rev: } & 0 \\
\text { Date: } & 07 / 22 / 88 \\
\text { Page: } & 4 \text { of } 5
\end{aligned}
$$

This will all be done on a frame by frame basis for each photograph taken. Instant pictures will also have the information recorded directly on the picture.

4.8 A camera pass is required for each photographer on the project. Requests for camera passes are initiated by the appropriate WAG Manager or the FSS Manager, approved by the Program Manager, and transmitted to Energy systems by the Project Administrative Supervisor. Requests are sent to the attention of the ORNL Field Activities Coordinator and should include, as a ruinimum, the name of the photographer and the reason for requesting the pass. Only personnel with approved photo passes will possess and transport photographic equipment on ORNL's facility.

4.9 Access to photographs is limited to project and Enelgy Systems personnel. Access to negatives and requests for adiitional prints is coordinated through the Project Administrative Supervisor.

4.10 Photographs taken by project personnel are for official project use. Use of photographs in oral presentations at open meetings, published papers, or any other off-project uses requires the written authorization of the Energy Systems RI/FS Project Manager.

\subsection{RESPONSIBILITIES}

The Program Manager is responsible for ensuring overall compliance with this procedure.

The WAG manager is responsible for determining the field photography requirements associated with the WAG RI. The WAG manager is also responsible for overseeing compliance with the recordkeeping. documentation requirements required by the ORNL permit and the RI/FS project requirements, and for selecting and interpreting the photography used in the RI report.

The FSS Manager is responsible for obtaining necessary permits and photographic equipment and supplies, for issuing such equipment and for processing film.

The individual designated as photographer is responsible for taking photographs in accordance with these procedures and for obtaining the necessary information to document the photography.

The Field $Q C$ supervisor is responsible for inspecting field activities to determine compliance with these procedures. 
Attachment B, Part B - RCRA Specific Comments Response 37

$$
\begin{aligned}
19118 \text { PP: } & 1110.1 \\
\text { Rev: } & 0 \\
\text { Date: } & 07 / 22 / 88 \\
\text { Page: } & 5 \text { of } 5
\end{aligned}
$$

The Project Alministrative Supervisor is responsible for the control of photographs and negatives in the office.

\subsection{REFERENCES}

None.

\subsection{ATTACHMENTS}

None. 
Attachment B, Part B - RCRA Specific Comments Response 38

ORNL RI/FS PROJECT JOB 19118

\section{PROJECT PROCEDURE REVISION ORDER}

1. PROCEDURE TO BE CHANGED:

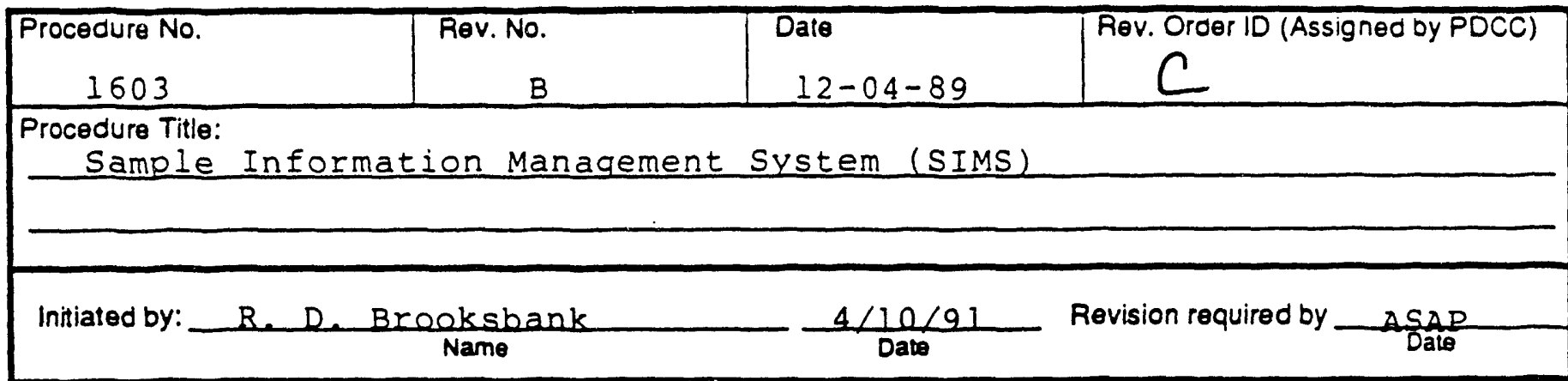

\section{DESCRIPTION OF CHANGE:}

Section 4.1 .6 , second paragraph:

Delete the third sentence in its entirety.

Section 4.2.5, Chain of Custody

Second paragraph, second sentence reads:

The samples must be transferred..., but no later than 24 hrs.

Change sentence to read:

The samples must be transferred... after collection.

\section{REASON FOR CHANGE}

To close Corrective Action Request Numbers 91-01 and 91-02.

4.

17 APPROVED

MODIFIED (as noted)
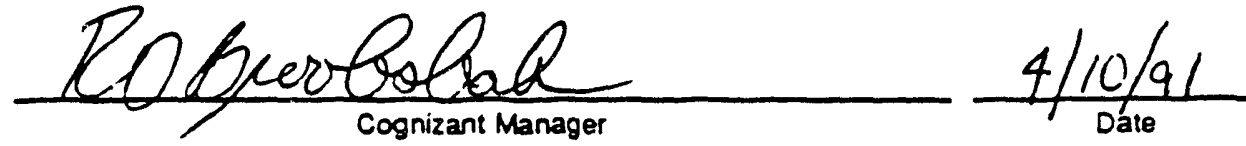

DISAPPROVED

5.

REVIEWED:
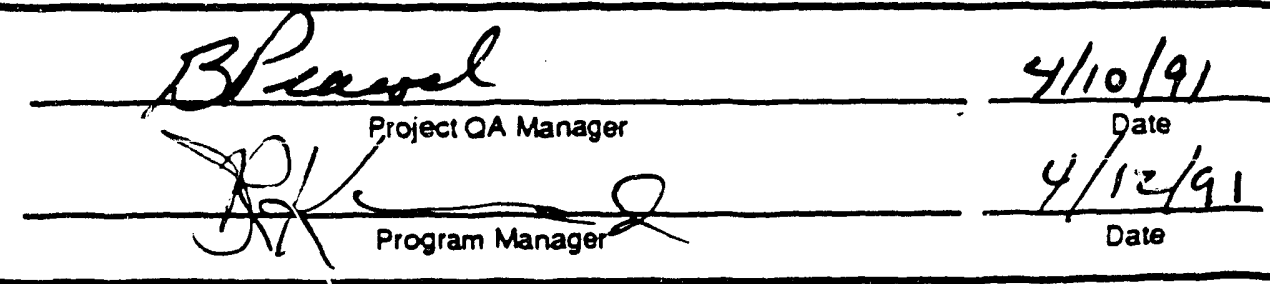

APPROVED: 


\author{
19118 PP: 1603 \\ Rev: $B$ \\ Page: 2 of 3 \\ Revision Order ID: C
}

The information recorded on the sample label (with the exception of sampler's signature) must also be recorded in the field logbook. In particular, the serialized sample label number must be linked to the sample number. The field logbook will serve as the prime sample tracking mechanism in the manual system.

\section{other Physical Evidence}

Sample labels are also used for labeling and tracking physical evidence other than sample jars, such as charts, or air and soil gas sample collection tubes or bags. If a label cannot be applied, a unique identifying number should be etched, written, scratched, or marked on the item (55-gal drum, pressure vessel, etc.) and the information required for a sample label recorded in the logbook. A completed sample tag can be forwarded with the item for analysis in a clear plastic bag, attached to the item by whatever means work.

Prior to decontamination of the sample, the sample label should be covered with clear tape to avoid smearing the entries. If the samples are enclosed in an outer package (paint can, core box), a duplicate (but not serialized) sample label should be filled out, attached to the outer container, and covered with clear, waterresistant tape.

\subsubsection{Chain of Custody Record}

The possession of samples or other physical evidence shall be traceable from the time they are obtained until they are introduced as evidence in legal proceedings. RFA forms (Attachment 1) are used to trace samples. A lapse in $C O C$ is usually grounds for the data relating to the sample to be dismissed as "tainted."

Samples taken for the ORNL RI/FS project must remain in the possession of the field investigator until transferred to the ALS representative or to the possession of the FSS manager. The samples must be transferred to the ALS representative to transport as soon as possible after collection, lates If samples must be retained overnight, the samplers must surrender custody of the sample to the FSS Manager, using the RFA form. The FSS Manager is responsible for securing and maintaining samples and transferring custody to the ALS as soon as practical.

PP1603_REVB.WP 
Attachment B, Part B - RCRA Specific Comments Response 38

$\begin{aligned} 19118 \text { PP } & 1603 \\ \text { Rev: } & B \\ \text { Page: } & 3 \text { of } 3 \\ \text { Revision Order ID: } & \end{aligned}$

- Prefix (CT) to identify module (automatic entry)

- User I.D. (scanned Martin Marietta badge number of employee relinquishing custody)

- Date and time for transaction (automatic entry)

- Container ID (scanned from label on container)

- Location of origin (scanned from menu card)

- Location for transfer (scanned from menu card)

- Custodian ID (scanned Martin Marietta badge number of individual receiving samples)

When a custody transfer involves placing samples into storage (e.g., interim or archival storage), all sample containers shall be stored in secure areas designated for that purpose. Access to storage facilities shall be strictly controlled by the Field services and support (FSS) Manager and monitored by the Field Environmental Safety and Health Supervisor.

Custody transfers outside the BNI Team and use of manual COC must be performed in accordance with the provisions of this procedure using the RFA form shown in Attachment 1.

\section{1 .6 Shipment of samples to the ALS}

The Shipping officer (SO) must ensure that samples are packaged, labeled, marked, placarded, and have appropriate shipping papers, in accordance with the provisions of the PP 1260 series, "Hazardous Materials Transportation."

The final bar code container transfer transaction prior to shipping (e.g., custody transfer to vehicle driver) must include a "shipping event" indicator. Prior to actual shipping, the FSC must ensure that the bar code reader (see Paragraph 4.1 .7 below) is uploaded to the FOF microcomputer (PC) and that an RFA form is generated. The FSS lander mutsignandate the eompletedorinding an a thos The FS Manager must ensure that the completed form is attached to the shipping papers. The shipment of the samples to the ALS laboratory facilities may then be executed.

PP1603_REVB.WP 
Attachment B, Part B - RCRA Specific Comments

Response 38

ORNL RI/FS PROJECT

JOB 19118

\section{PROJECT PROCEDURE REVISION ORDER}

1. PROCEDURE TO BE CHANGED:

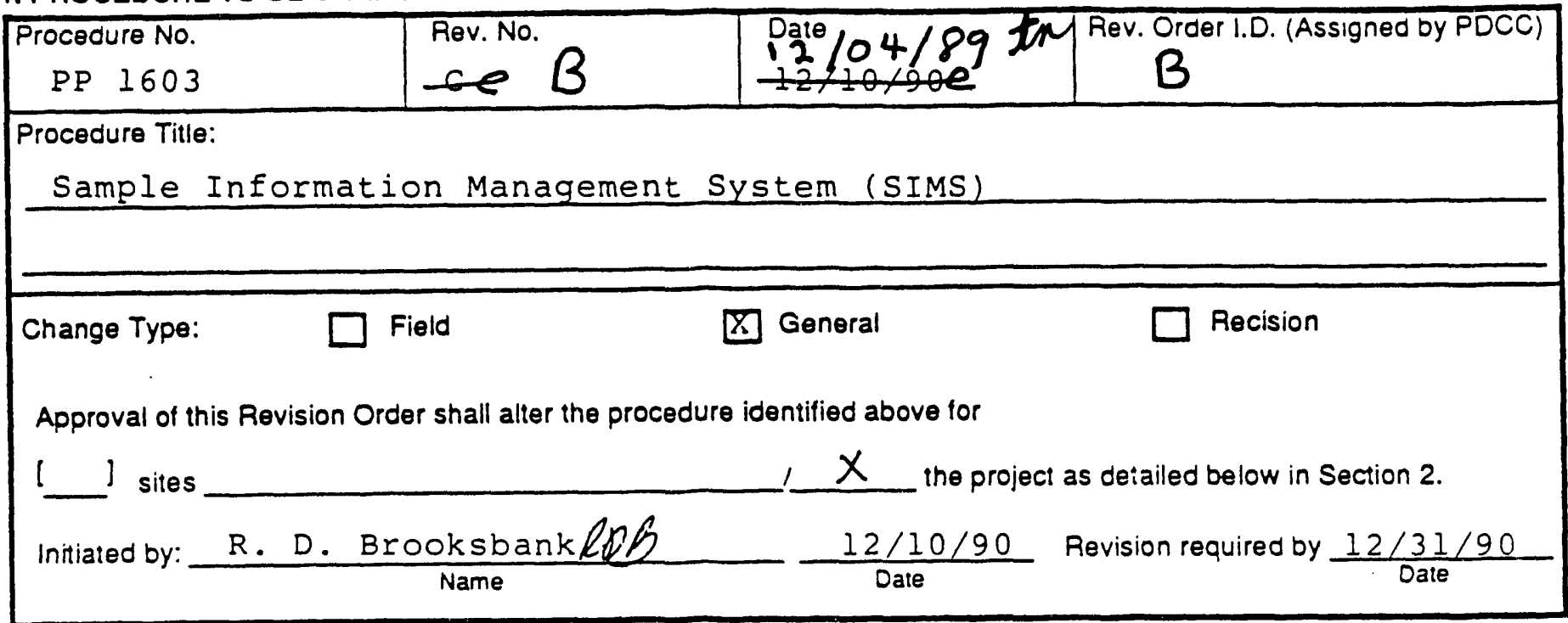

2. DESCRIPTION OF CHANGE:

1. Change 3rd sentence of paragraph 4.1 .1 to read as follows:

Requests are to be made and forwarded to the ALS within 7 days after receipt by the Laboratories Manager.

3.

APPROVED

MODIFIED (as noted)

DISAPPROVED

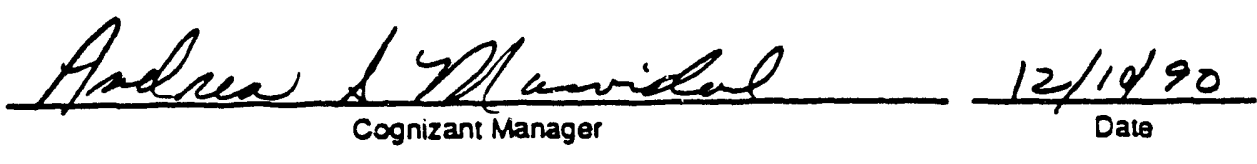

4.

REVIEWED:

30 ent

QA Manager $\frac{12 / 10 / 90}{\text { Date }}$

CONCURRED:
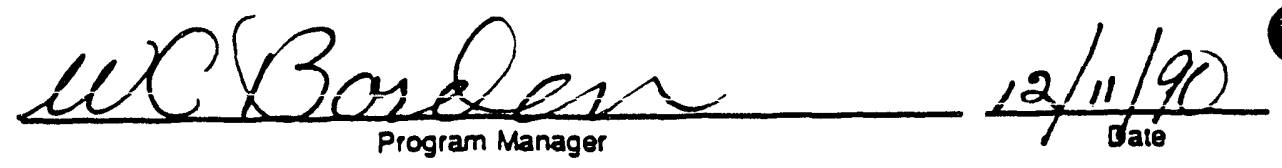
1. PRocedure to be changed:

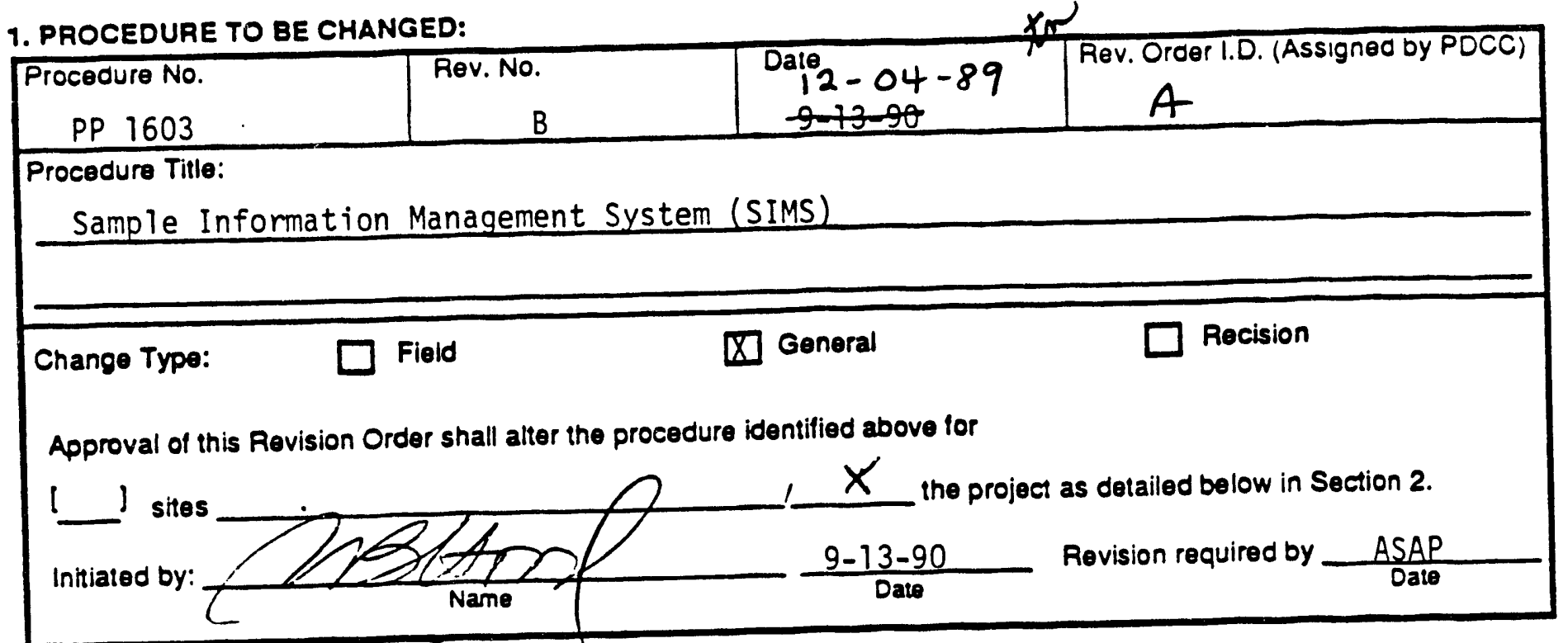

\section{DESCRIPTION OF CHANGE:}

Change paragraph 4.2.6 Custody Seals to read:

Containers used for the purpose of transporting samples from the sample sites will be sealed with custody seal tape. The sample custodian will take custody of the container at the Interim Storage Facility, inspect to assure seals have not been broken, note any-breaks on the RFS and reseal the container. Shipping and packaging for transport to RSL will be in accordance with PP 1260 series.

3.

D. APPROVED

MODIFIED (as noted)

DISAPPROVED

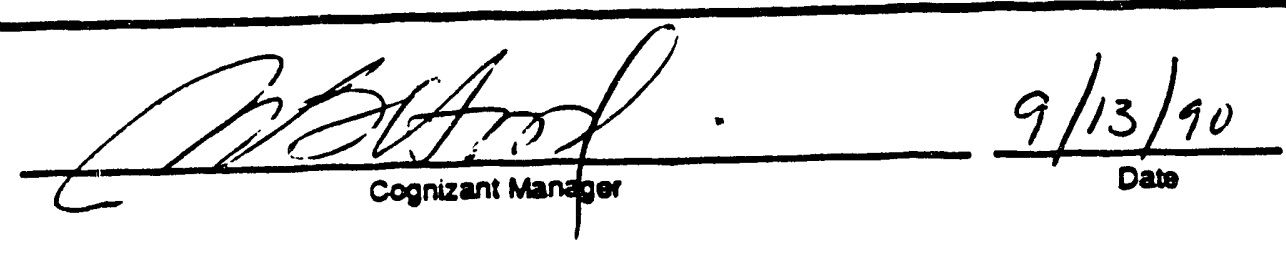

4.

REVIEWED:

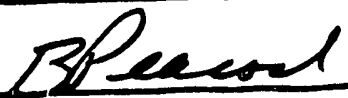

$\frac{10 / 18 / 90}{\text { Dato }}$

CONCURRED:
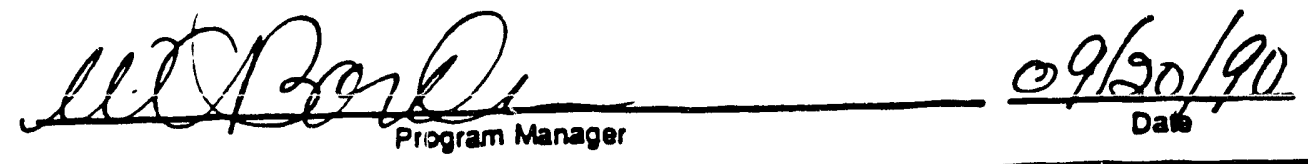
Attachment B, Part B - RCRA Specific Comments Response 38

\section{TITIE:}

DOCUMENT NUMBER: 1603

REVISION NUMBER: B

DATE :
SAMPLE INFORMATION MANAGEMENT SYSTEM (SIMS)

$12 / 04 / 89$

PROJECT: ORNL RI/FS

JOB NO.: 19118

PREPARED:

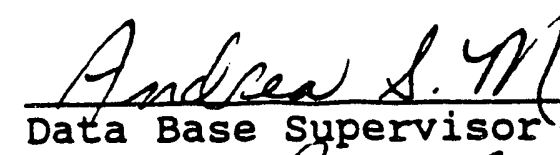

$\frac{1 / 29 / 90}{\text { Date }}$

REVIEWED:

Buffecoul

Project Quality Assurance Supervisor

$\frac{1 / 29 / 90}{\text { Date }}$

APPROVED:

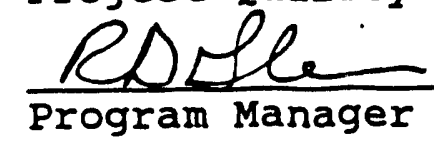


Attachment B, Part B - RCRA Specific Comments Response 38

$\begin{aligned} 19118 \text { PP: } & 1603 \\ \text { Rev: } & \text { B } \\ \text { Page: } & 1 \text { of } 14\end{aligned}$

SAMPLE INFORMATION MANAGEMENT SYSTEM

\subsection{PURPOSE}

This procedure provides methodology for use of the sample Information Management system (SIMS) protocols for sample accumulation and tracking functions.

\subsection{SCOPE}

This procedure applies to all ORNL RI/FS project field sampling activities.

\subsection{DEFINITIONS}

Custody - A sample or other physical evidence is considered to be in custody if the possession of the sample can be traced from the time of collection until the sample analytical data is introduced as evidence in legal proceedings. For example:

- it is in the field investigator's or the transferee's possession: or

- it is in the field investigator's or the transferee's view, after being in his/her physical possession; or

- it was in the field investigator's or the transferee's physical possession, and then he/she secured it to prevent tampering; or

- it is placed in a designated secure area.

Sample - Physical evidence collected from a facility/site or the environment for monitoring purposes. Evidence includes collected environmental material, photographs, remote sensing data, and in situ field measurements and analyses, whether taken directly from a collected sample or made with field instruments.

Sample Identification Documents (Sample Documents) - Any form, report, or record prepared to maintain chain of custody (COC), including, but not limited to, the following:

- Request for Analysis (RFA) Form (Attachment 1)

PP1603_REVB.WP 
Attachment B, Part B - RCRA Specific Comments

Response 38

$$
\begin{aligned}
19118 \text { PP: } & 1603 \\
\text { Rev: } & \text { B } \\
\text { Page: } & 2 \text { of } 14
\end{aligned}
$$

- Sustody Seals (Attachment 2)

- Field rotebooks (see PP 1631, "Logbook Protocols")

- Photographic logs (see PP 1110.1, "Field Photography and Control")

- Forms used in commercial transport (i.e., Federal Express Airbills and Hazardous Cargo Manifests! of samples for analysis at other than the Analytical Laboratory subcontractor (ALS) facility

- specialized sample identification and management forms or modified RFA forms required by the WAG field sampling plan or QA project procedures

\subsection{PROCEDURES}

The following major activity functions shall be completed for each sample, or group of samples, taken.

\subsection{ELECTRONIC DATA TRANSFER}

\subsubsection{Laboratory Notification}

The sampling event must be initiated by the WAG manager's request for analysis, issued to the Laboratories Manager and the Field Sample Coordinator (FSC) at the RI/FS project Field operations Facility (FOF). The request is made on a sample Event Initiation Request (SEIR) form (Attachment 3). Requests are to be made and forwarded to the ALs at least seven working days prior to the anticipated sampling event. It is the responsibility of the Laboratories Manager to notify the ALs by providing them a copy of the SEIR form. In addition, the FSC and Laboratories Manager must ensure that an adequate stock of the various containers and preservative reagents is continuously available for all sampling events.

\subsubsection{Container Preparation}

Upon receipt of the SEIR form, the FSC must coordinate preparation of all sample containers necessary to fulfill the SEIR form requirements. Each container must be equipped with a unique bar code label consisting of four digits to identify sampling location and two digits to ensure uniqueness in container identification. All containers with the same first 
Attachment B, Part B - RCRA Specific Comments Response 38

\author{
19118 PP: 1603 \\ Rev: $B$ \\ Page: 3 of 14
}

four digits comprise a sample set (to be taken from the same location). Prior to sampling, the close support Laboratory (CSI) Supervisor will affix a second bar code label to each container identifying the preservative added to the container.

Using bar code readers and software, the FSC will prepare an ASCII file of container preparation parameters for each sample to be collected. The file consists of a prefix (CP) to indicate the container preparation software module; a user I.D. (Martin Marietta badge number for the employee/scanned from badge); date and time of transaction (automatic entry); container ID (scanned from label on container); and pay item numbers to identify requested analyses (scanned from bar coded menu cards). All such ASCII files are to be uploaded from bar code readers to personal computers at the FOF and then transmitted to BNI's Digital VAX computer system using modems and communications software. All bar code files are loaded into ORACLE tables for sample tracking. Electronic tracking is begun by matching uploaded container preparation files to SEIR forms.

\title{
4.1.3 Preparation for Sampling
}

A designated sampling team member must obtain those containers, preservatives, packaging materials, coolers, bar code readers, etc., necessary for that day's scheduled sampling events from the FSC.' All coolers will contain a bar code label identifying sample location which consists of four digits (matching the first four digits on all container labels belonging to that sample set).

Prior to initiation of sampling activities, the names of all sampling team members must be entered into the field logbook. If team members leave the sampling area at any time during the shift or if new team members arrive, the times of departure/arrival must also be entered into the field logbook.

Menu cards for all data collection are prepared and issued to sample team leaders prior to sampling. Menu cards include bar code labels specifying sampling location; sample matrix; sample type (e.g., regular, field blank, duplicate, etc.); and depth intervals for soil samples.

All bar code labels and menu cards are printed at the FOF using personal computers and printers and graphics software packages. All labels used on menu cards or affixed to containers and coolers will be checked for accuracy using the label check option

PP1603_REVB.WP 


$$
\begin{aligned}
19118 \text { PP: } & 1603 \\
\text { Rev: } & \text { B } \\
\text { Page: } & 4 \text { of } 14
\end{aligned}
$$

available on the portable bar code readers. Should any labels fail to read correctly, replacements will immediately be printed and substituted for those failing the check.

\subsubsection{Sample Collection}

In preparation for the collection of a sample, a designated team member must use the sample structure Module of BNI's bar code software to record the following information:

- Prefix (SS) to identify module for transaction (automatic entry)

- Team member's (individual using the bar code reader or the sampler) Martin Marietta badge number (scanned from badge)

- Date and time stamp for transaction (automatic entry)

- Sampling location site (e.g., well/hole number - scanned from menu card)

- Container ID (scanned from label on container)

- Sample matrix code (scanned from menu card)

- Preservatives added or NONE (scanned from container label)

- Sample Type (scanned from menu card)

- Depth interval (soil samples only/scanned from menu card)

Upon successful completion of the foregoing bar code reader entries, the designated team member must enter the container identification (bar code label on the container) into the field logbook. Immediately after this entry, the team member must make those entries required by the provisions of PP 1631, "Logbook Protocols."

\subsubsection{Custody Transfer}

The container transfer module of the bar code software must be used each time that the containers change hands to ensure a complete record of COC. The CT files contain:

- Prefix (CT) to identify module (automatic entry) 
Attachment B, Part B - RCRA Specific Comments Response 38

$$
\begin{aligned}
19118 \text { PP: } & 1603 \\
\text { Rev: } & \text { B } \\
\text { Page: } & 5 \text { of } 14
\end{aligned}
$$

- User I.D. (scanned Martin Marietia badge number of employee relinquishing custody)

- Date and time for transaction (automatic entry)

- Container ID (scanned from label on container)

- Location of origin (scanned from menu card)

- Location for transfer (scanned from menu card)

- Custodian ID (scanned Martin Marietta badge number of individual receiving samples)

When a custody transfer involves placing samples into storage (e.g., interim or archival storage), all sample containers shall be stored in secure areas designated for that purpose. Access to storage facilities shall be strictly controlled by the Field services and support (FSS) Manager and monitored by the Field Environmental safety and Health supervisor.

Custody transfers outside the BNI Team and use of manual coc must be performed in accordance with the provisions of this procedure using the RFA form shown in Attachment 1.

\subsubsection{Shipment of Samples to the ALS}

The Shipping officer (SO) must ensure that samples are packaged, labeled, marked, placarded, and have appropriate shipping papers, in accordance with the provisions of the PP 1260 series, "Hazardous Materials Transportation."

The final bar code container transfer transaction prior to shipping (e.g., custody transfer to vehicle driver) must include a "shipping event" indicator. Prior to actual shipping, the FSC must ensure that the bar code reader (see Paragraph 4.1 .7 below) is uploaded to the FOF microcomputer (PC) and that an RFA form is gentrated. The FSS Manager must sign and date the completed form, indicating an authorized BNI Team request for analysis. The FSS Manager must ensure that the completed form is attached to the shipping papers. The shipment of the samples to the ALs laboratory facilities may then be executed.

\subsubsection{Uploading Bar Code Readers to Computers}

PP1603_REVB.WP 


$$
\begin{aligned}
19118 \text { PP: } & 1603 \\
\text { Rev: } & \text { B } \\
\text { Page: } & 6 \text { of } 14
\end{aligned}
$$

Information collected by each hand-held bar code reader must be transferred (uploaded) to RI/FS project PCs at the end of each work shift and should be uploaded more frequently if large numbers of high volume transactions are executed (e.g., at the lunch break and at the end of the shift).

\subsubsection{Information Backup}

Transactions uploaded to RI/FS project PCs during a single calendar day (midnight to midnight) are written to a single flexible disk (floppy) and journalized on the internal hard disk that is unique to that particular PC and for that particular day. This backup function is executed automatically at the completion of each individual bar code reader upload transaction. The FSC must ensure that each day's uploaded data for a given PC is stored on a separate floppy disk that is free of any other data.

At the conclusion of each working day, the PC operator must print the directory for each floppy disk. The PC operator must also label each printed directory with location, PC identifier, and the date. The PC operator must then sign each such record to attest to the accuracy of contained information and file the labeled floppy disk in a secure location.

Upon completion of the foregoing backup operations, the PC operator will use modems and communications software to upload that day's transactions from the PC to the VAX computer system located at Bechtel's Oak Ridge office. If for any reason the data communications links cannot be established, the PC operator must back up the floppy disk (copy entire contents onto another floppy disk) and forward the backup copy of the floppy disk to the Data Base Supervisor. The Data Base Supervisor must ensure that the data contained on the floppy disk(s) are loaded onto the VAX computer sytem (RI/FS project data base) within 24 hours of receipt of the diskette. The Data Base supervisor must notify the FSS Manager if any data could not be successfully loaded into the RI/FS data base or if any data should look suspect. Necessary changes, additions, or deletions must be documented on a Data Base Change Request form (Attachment 5) before actual alteration of any data. Logbook entries should be consulted prior to approval of change requests.

\subsection{MANUAL SAMPIE MANAGEMENT AND CHAIN OF CUSTODY}

PP1603_REVB.WP 


$$
\begin{aligned}
19118 \text { PP: } & 1603 \\
\text { Rev: } & \text { B } \\
\text { Page: } & 7 \text { of } 14
\end{aligned}
$$

\subsubsection{General}

Manual methods for the management of samples will be used whenever bar code equipment is unavailable or when the automated procedures are impractical. Sample identification, field records, and COC records will be recorded in waterproof, nonerasable ink. If errors are made in any of these documents, BNI Team members should make correction by crossing a single line through the error, entering the correct information, and initialing and dating the correction. If possible, corrections should be made by the individual who made the error, or the individual who made the error should be advised of the correction.

\subsubsection{Sample Identification}

The provisions of section 4.1 are followed in obtaining sample containers, in reserving analytical capacity in the laboratory, and in preparation for field sampling.

\subsubsection{Field Measurements}

Those samples that consist of field measurements and analyses, such as temperature, $\mathrm{pH}$, Eh, conductivity, flow measurements, geophysical measurements, radiological measurements, surveying measurements, and ambient air characterization measurements (i.e., oxygen/combustible gas levels, organic vapor levels, concentrations of toxic gases, etc.), are identified by recording the information in a bound field logbook (which may contain specialized forms). Temperature, $\mathrm{pH}$, and specific conductivity are also recorded on Sampling point Location forms (Attachment 4) which are provided to the Data Base Supervisor after each sampling event.

\subsubsection{Sample Identification}

\section{Environmental Samples}

Sample identification forms (Attachment 6) are provided to the BNI Team by the FSC, along with sample containers, preservatives, and other items for sample collection and management.

Samples, other than those collected for in situ field measurements or analyses, are identified by using a standard sample field tag (Attachment 6) which is attached to the sample container. In some cases, such as rock cores, the sample field 


$$
\begin{aligned}
19118 \text { PP: } & 1603 \\
\text { Rev: } & \text { B } \\
\text { Page: } & 8 \text { of } 14
\end{aligned}
$$

label may have to be included with or wrapped around the sample. The sample field labels are sequentially numbered and are accountable documents after they are completed and attached to a sample or other physical evidence.

The sample field label can be filled out prior to obtaining the sample, leaving only the date/time and sampler's signature blank. The sample field tag (Attachment 6) is completed with the following information:

- Project Name: ORNL RI/FS (specify WAG or project name, such as "WAG 10 P\&A").

- Project Number: Use the BNI project number and work release number (e.g., 19118-X022).

- Sample Number: A unique identifying number consisting of numbers and letters. The sample number is determined by the WAG Manager.

- Collection Date/Time: Use the following notation: MO/DA/YR/HR : MN : SC

where: $M O$ is a two-digit numeral representing the month. Use zeros as the first digit where appropriate.

$D A$ is a two-digit numeral representing the day. Use zeros as the first digit where appropriate.

$Y R$ is a two-digit numeral representing the last two digits of the year.

Either slashes (/) or dashes (-) may be used to separate the dates. Colons are used to separate the time elements,

where: HR is a two digit numeral representing the hour. Use military (24-hr) notation, with the hours after noon represented by the numbers 13 ( 1 p.m.) through 23 (11 p.m.). Midnight is expressed as 00:00 hours, noon as 12:00. Use zeros as the first digit where appropriate.

$M N$ is a two-digit numeral representing the minutes. Use zeros as the first digit where appropriate. 
Attachment B, Part B - RCRA Specific Comments Response 38

$$
\begin{aligned}
19118 \text { PP: } & 1603 \\
\text { Rev: } & \text { B } \\
\text { Page: } & 9 \text { of } 14
\end{aligned}
$$

SC is a two-digit numeral representing the seconds. Use only when required. Use zeros as the first digit where appropriate.

- Collector's Name: PRINT your name [if more than one person is collecting samples, provide the actual collector's name, the name of the person bearing on-site responsibility for the sampling effort, or the names of the entire sampling team (usually two names)]. Whenever possible, add the sampler's ORNL identification badge number.

- Sample Location: Provide sufficient detail to enable the same location to be resampled at a later date.

- Sample Type/Depth/Description: The sample is either a grab or a composite. Specify depth taken for groundwater, surface water, soils, lagoons, or impoundments, waste piles, etc. Describe the media sampled (groundwater, etc.) and briefly specify hazards (high hazard, rad hazard, environmental sample). Specify the sample as a field or travel blank (FB or TB), a duplicate (DUP), or a non-QC replicate (REP). QC samples, such as replicates, submitted to the laboratory at the direction of the Laboratoris Manager are an exception and will be submitted as a "blind" to the laboratory, marked as a normal sample.

- Preservative: The type of preservative used will be filled in by the close support Laboratory (CSI) supervisor. See PP 1632, "Sample Containers and Preservatives."

- Request for Analysis number: The type of analysis is determined by the WAG Manager in conjunction with the Laboratories Manager prior to collecting the sample. The RFA number corresponds to the RFA form (Attachment 1) filed in by the laboratory. The type of preservative, size and type of jar, and RFA number are interdependent. The sampler fills the container with the correct sample based on the three items.

Bottle of Used to indicate samples in more than one container, such as a water sample in two one-liter bottles.

Filtered/Nonfiltered Check the appropriate item.

The information recorded on the sample label (with the exception of sampler's signature) must also be recorded in the field 
Attachment B, Part B - RCRA Specific Comments Response 38

$\begin{aligned} 19118 \text { PP: } & 1603 \\ \text { Rev: } & \text { B } \\ \text { Rage: } & 10 \text { of } 14\end{aligned}$

logbook. In particular, the serialized sample label number must be linked to the sample number. The field logbook will serve as the prime sample tracking mechanism in the manual system.

\section{other Physical Evidence}

Sample labels are also used for labeling and tracking physical evidence other than sample jars, such as charts, or air and soil gas sample collection tubes or bags. If a label cannot be applied, a unique identifying number should be etched, written, scratched, or marked on the item (55-gal drum, pressure vessel, etc.) and the information required for a sample label recorded in the logbook. A completed sample tag can be forwarded with the item for analysis in a clear plastic bag, attached to the item by whatever means work.

Prior to decontamination of the sample, the sample label should be covered with clear tape to avoid smearing the entries. If the samples are enclosed in an outer package (paint can, core box), a duplicate (but not serialized) sample label should be filled out, attached to the outer container, and covered with clear, waterresistant tape.

\subsubsection{Chain of Custody Record}

The possession of samples or other physical evidence shall be traceable from the time they are obtained until they are introduced as evidence in legal proceedings. RFA forms (Attachment 1) are used to trace samples. A lapse in COC is usually grounds for the data relating to the sample to be dismissed as "tainted."

Samples taken for the ORNL RI/FS project must remain in the possession of the field investigator until transferred to the ALS representative or to the possession of the Fss manager. The samples must be transferred to the ALS representative to transport as soon as possible after collection, but no later than 24 hrs. If samples must be reti sed overnight, the samplers must surrender custody of the sample to the FSS Manager, using the RFA form. The FSS Manager is responsible for securing and maintaining samples and transferring custody to the ALS as soon as practical.

The RFA form (Attachment 1) is used to record the custody of all samples or other physical evidence collected and maintained by

PP1603_REVB.WP 
Attachment B, Part B - RCRA Specific Comments Response 38

$$
\begin{aligned}
19118 \text { PP: } & 1603 \\
\text { Rev: } & \text { B } \\
\text { Page: } & 11 \text { of } 14
\end{aligned}
$$

BNI Team personnel. This form also serves as a sample logging and tracking mechanism for the ALs sample custodian.

The following information must be supplied in the indicated spaces in detail to complete the RFA form:

- The project name and number

- The lab designation, previously determined by the Laboratories Manager, FSS Manager, and WAG Manager

- All designated signature blocks must be signed

- The sample number, sample matrix, container I.D., preservative, pay item numbers, requested testing programs, priority level, and $Q C$ level are included for each sample. only one sample is described on each line. This information is obtained from the sample labels, the container preparation module of the bar code transaction files, and the SEIR form.

The "special instructions" line is used to list any additional testing requested or to clarify above items. Whenever possible, samples requiring similar analyses should be listed on the same RFA form and shipped together. The "Possible Hazard Identification" line is used to alert laboratory personnel to take special precautions when opening the sample shipment package. If the sample is surrendered to an on-site laboratory, the name and employee number of the sample custodian for that laboratory must be entered in the "Samples Received By" slot.

The sample custodian maintains custody throughout the analytical process. When the sample is first received, the "condition on receipt" is noted. If the containers are broken, damaged, empty, or if custody seals have been broken (see below), the sample custodian will set the sample aside and inform the Laboratories Manager, who will contact the FSS Manager and WAG Manager to determine if analysis should proceed or if resampling is indicated. The sample custodian will complete the RFA form when analysis is completed and the sample is disposed and will return a copy of the completed form to the Laboratories Manager for distribution to the permanent project record.

\subsubsection{Custody Seals}

Samples will be sealed immediately upon collection, utilizing the custody seal shown in Attachment 2, bridging the lid/bottle gap,

PP1603_REVB.WP 
Attachment B, Part B - RCRA Specific Comments Response 38

$\begin{aligned} 19118 \text { PP: } & 1603 \\ \text { Rev: } & \text { B } \\ \text { Page: } & 12 \text { of } 14\end{aligned}$

so that the seal will be broken if the container is opened. Seals are also placed on boxes or coolers used to transport samples to the laboratory. Any time the custody seal is broken by investigators in the field to get access to the sample, a notation is made on the. RFA form, and a new seal applied. The laboratory sample custudian will note any broken custody seals and inform the SAI Coordinator.

\subsubsection{Request for Analysis}

- The RFA form (Attachment 1) is sent to the laboratory by FOF personnel. Information regarding the sample/container number, sample type/matrix, preservative, pay items, priority and $Q C$ levels is recorded on the RFA form under the direction of the Laboratories Manager. The information required can be taken from the bar code container preparation files for each container, wi.th the exception of priority and QC levels. The corresponding SEIR form provides that information.

RFA forms accompany the samples listed on the forms to trie laboratory.

\subsubsection{General Considerations}

A RFA form must be completed for all samples or physical evidence collected. A separate form is necessary for each laboratory utilized during the inspection or investigation and for the final destination of the sample (archived or disposed).

During the course and at the end of the field work, the WAG manager, Laboratories Manager, and Field QC Supervisor determine whether these procedures have been followed and if the collection of additional samples is required.

In general, BNI Team personnel must not accept samples from other sources (i.e., Energy systems, Rust Engineering, etc.) unless the sample collection procedures used are known to be acceptable, can be documented, and the sample coc can be established. Typically, the requirements can be met by having a BNI Team member observe the sampling effort. If such samples are accepted by BNI Team members, a standard sample tag containing relevant information and a RFA form must be completed for each set of samples.

When samples are split with a state or federal regulatory agency, the regulatory agency representative signs the RFA form. 
Attachment B, Part B - RCRA Specific Comments Response 38

$\begin{aligned} 19118 \text { PP: } & 1603 \\ \text { Rev: } & \text { B } \\ \text { Page: } & 13 \text { of } 14\end{aligned}$

\subsection{RESPONSIBILITIES}

The program Manager is responsible for ensuring overall compliance with this procedure.

The WAG managers are responsible for timely notification to the Laboratories Manager of sampling requirements and close coordination with the FSS Manager for authorization/approval for analysis of opportunistic samples.

T'ie Laboratories Manager is responsible for timely coordination with the ALS, for ensuring that the ALS responds to programmatic needs of the project in a timely and cost-effective manner, and for ensuring that the ALS utilizes the SIMS in accordance with this procedure and contractual directives.

The FSS Manager is responsible for field control of sample container stocks, storage/maintenance/issuance of bar code readers, and field data transfers.

The Data Base Supervisor is responsible for ensuring the quality and timeliness of SIMS and related data bases maintained on the VAX computer system and for technical inspections and surveillances of field and laboratory operations to ensure that data are collected in an appropriate and quality manner.

The Field QC supervisor is responsible for inspecting field activities to determine compliance with this procedure.

\subsection{REFERENCES}

(1) Oak Ridge National Laboratory, "ORNL SAS Data Sets," 1987.

(2) Eechtel National, Inc. Team, mroject Management Plan for the Oak Ridge National Laboratory Remedial Investigation/ Feasibility study," ORNL/RAP/Sub-87/99053/1, September 1987.

(3) Martin Marietta Energy systems, Inc., "Environmental Surveillance Procedure Quality Control Program," ESH/Sub/87-21706/1 DRAFT, September 1988 .

7.0 ATTACHMENTS

Attachment 1: Request For Analysis

PP1603_REVB.WP 
Attachment B, Part B - RCRA Specific Comments Response 38

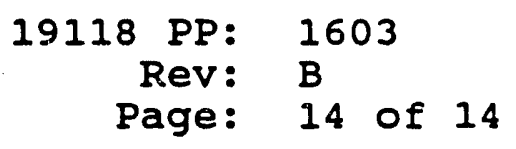

Attachment 2: Custody seal

Attachment 3: Sample Event Initiation Request

Attachment 4: Sampling Point Location Data Input Form

Attachment 5: Data Base Change Request Form

Attachment 6: Sample Field Label 


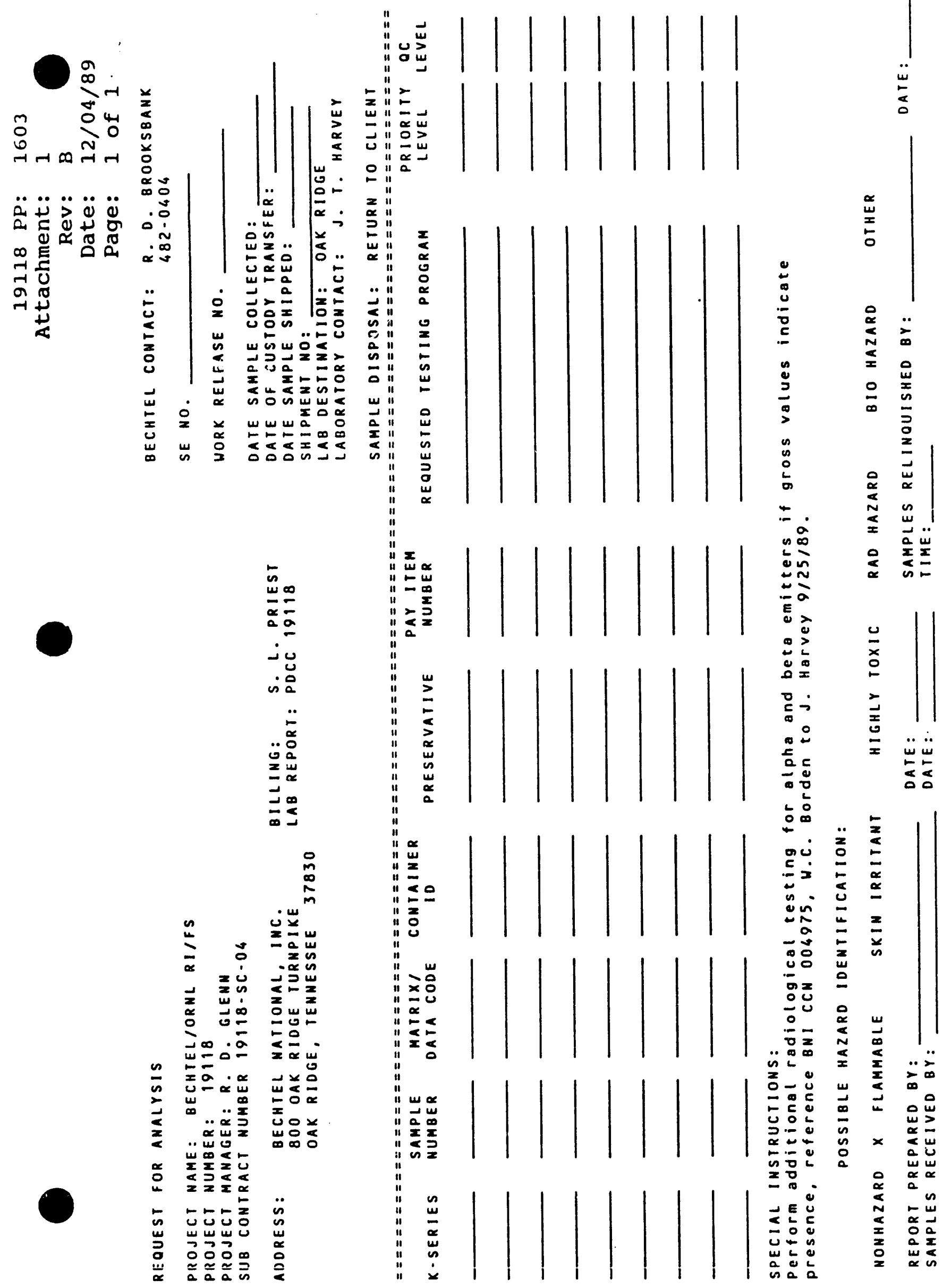


Attachment B, Part B - RCRA Specific Comments Response 38

$\begin{aligned} 19118 \text { PP: } & 1603 \\ \text { Attachment: } & 2 \\ \text { Rev: } & \text { B } \\ \text { Date: } & 12 / 04 / 89 \\ \text { Page: } & 1 \text { of } 1\end{aligned}$

CUSTODY SEAL

I A B O

S A Y P I E 8

PYI603_REVE.T̄P 
Attachment B, Part B - RCRA Specific Comments Response 38

19118 PP: 1603

Attachment: 3

Rev: $B$

Date: $12 / 04 / 89$

Page: 1 of 1

\section{SAMPLE EVENT INITIATION REQUEST}

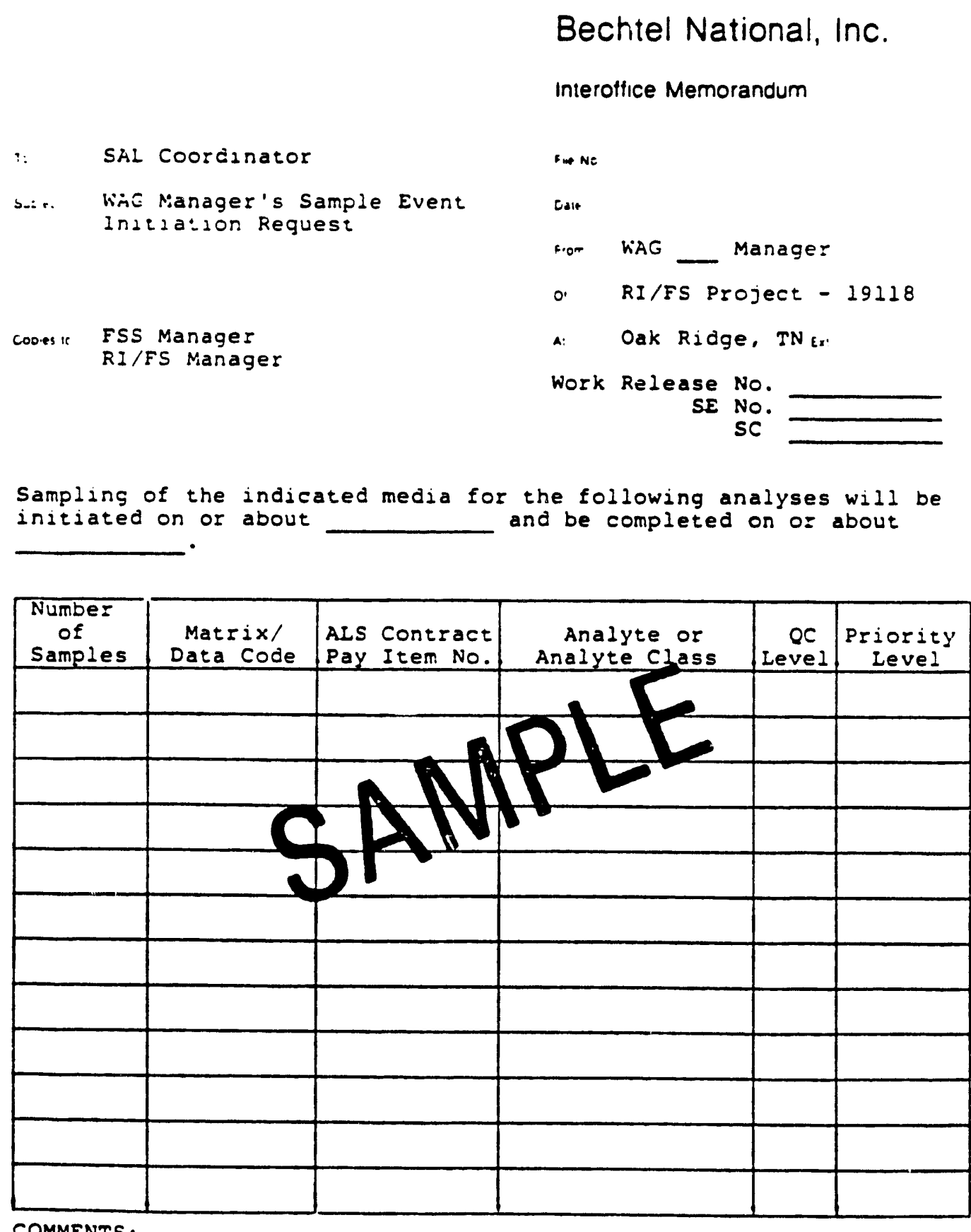

COMMENTS : 
Attachment B, Part B - RCRA Specific Comments

Response 38

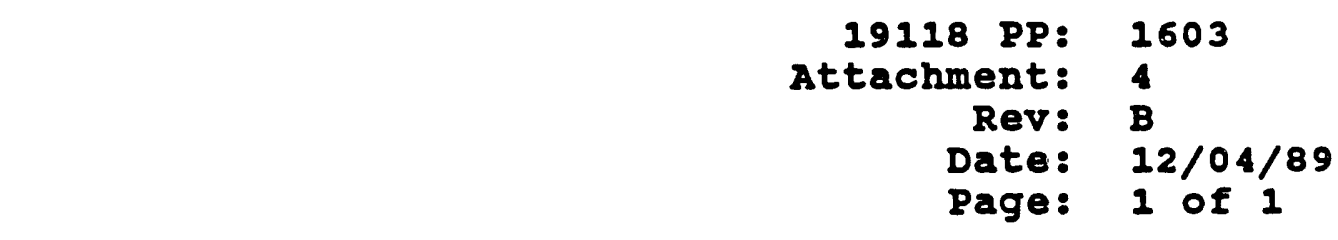

SAMPIING POINT LOCATION DATA INPUT FORM

RI/FS DATA INPUT FORY/88V105

SAMPLING POINT LOCATION DATA

WAG

LOCATION ID

GRID SYSTEM

EASTING

NORTHING

GROUND SURFACE ELEVATION - - CIVIL SURVEY DATE

SAMPLE COLLECTED? — $Y$ - N SAMPLE TYPE (MATRIX)

SAMPLE DATE

\begin{tabular}{|c|c|c|c|c|}
\hline & TIME 1 & TIME 2 & TIME 3 & TIME 4 \\
\hline & - & - & - - & - - \\
\hline $\mathbf{P H}$ & & & & \\
\hline PECIFIC CONDUCTANCE & & & & \\
\hline TEMPERATURE & & & & \\
\hline
\end{tabular}

DATA COLLECTED BY (NAME)

REMARRS

COMPLETED DATA COLLECTION FORM CHECRED BY:

VERIFICATION CODE:

UNVERIFIED

- VERIFIED/LIMITED USF

VERIFIED/INVALID

VERIFIED/VALID

WORK RELEASE NO. 
Attachment B, Part B - RCRA Specific Comments Response 38

$\begin{aligned} 19118 \text { PP: } & 1603 \\ \text { Attachment: } & 5 \\ \text { Rev: } & \text { B } \\ \text { Date: } & 12 / 04 / 89 \\ \text { Page: } & 1 \text { of } 1\end{aligned}$

DATA BASE CHANGE REQUEST FORM

DATA FILE NAME

DATE OF DATA COLLECTION _ _

CHANGE REQUESTED BY (NAME)

ITEM TO BE CHANGED

EXISTING ENTRY

CORRECTED ENTRY

CORRECTION APPROVED BY (NAME)

DATE OF CHANGE REQUEST 
Attachment B, Part B - RCRA Specific Comments

Response 38

19118 PP:

Attachment:

Rev:

Date:

Page:
1603

6

B

$12 / 04 / 89$

1 of 1

BAMPLE FIELD IABEL

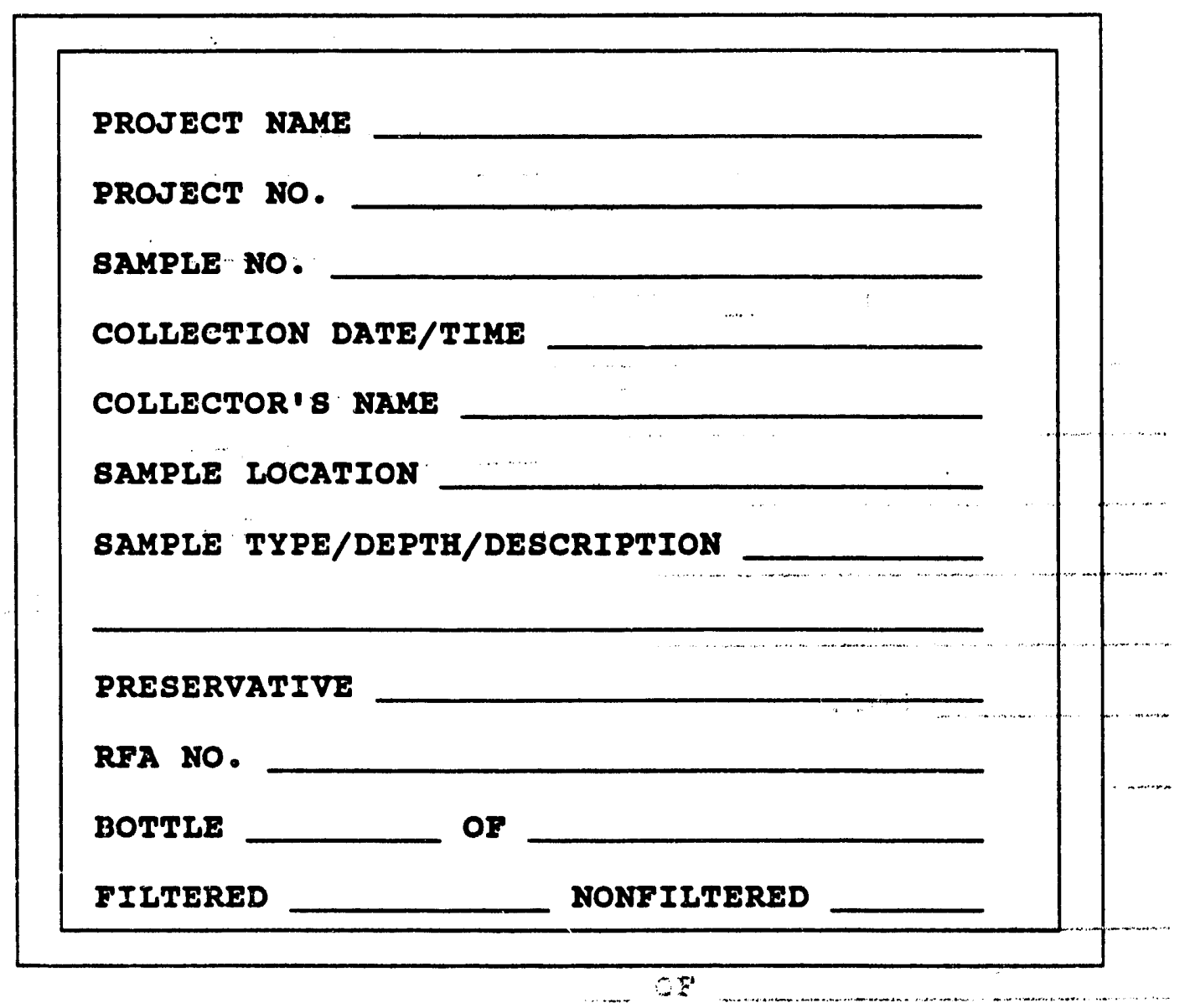

POH- $\triangle+\triangle B R$ 

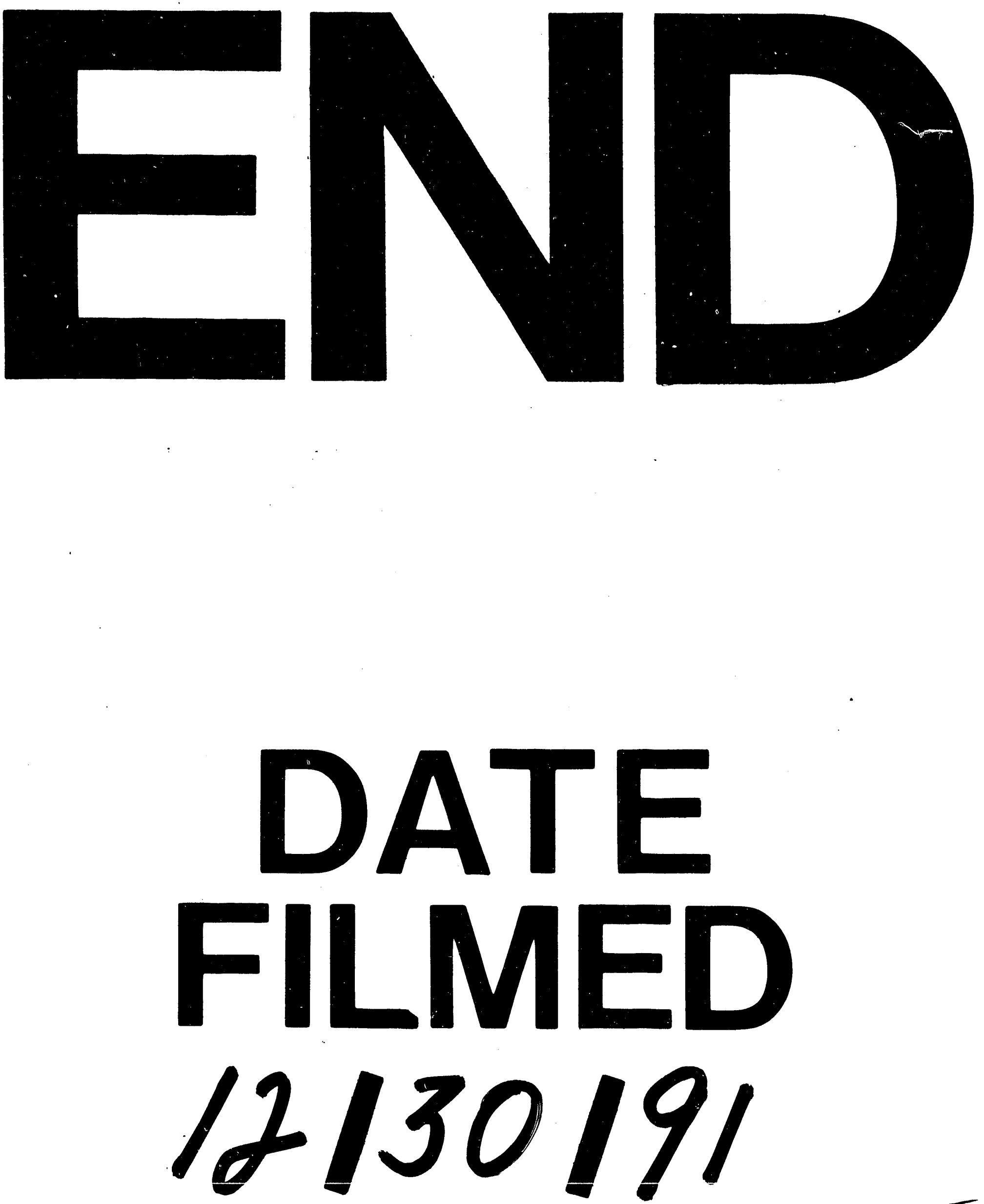
